Systems Engineering for Power IV

\title{
Analysis and Characterization of Security Regions in Power Systems
}

January 1979

U.S. Department of Energy

Assistant Secretary for Energy Technology

Division of Electric Energy Systems

Under Contract No. C-77-C-01-5011 


\section{DISCLAIMER}

This report was prepared as an account of work sponsored by an agency of the United States Government. Neither the United States Government nor any agency Thereof, nor any of their employees, makes any warranty, express or implied, or assumes any legal liability or responsibility for the accuracy, completeness, or usefulness of any information, apparatus, product, or process disclosed, or represents that its use would not infringe privately owned rights. Reference herein to any specific commercial product, process, or service by trade name, trademark, manufacturer, or otherwise does not necessarily constitute or imply its endorsement, recommendation, or favoring by the United States Government or any agency thereof. The views and opinions of authors expressed herein do not necessarily state or reflect those of the United States Government or any agency thereof. 


\section{DISCLAIMER}

Portions of this document may be illegible in electronic image products. Images are produced from the best available original document. 
Available from:

National Technical Information Service (NTIS)

U.S. Department of Commerce

5285 Port Royal Road

Springfield, Virginia 22161

Price: Printed copy: $\$ 9.00$

Microfiche: $\quad \$ 3.00$ 
Systems Engineering for Power IV

\section{Analysis and Characterization of Security Regions in Power Systems}

January 1979

Prepared by:

McGill University

Montreal, Quebec

To be presented at:

Engineering Foundation Conference

Tidewater Inn

Easton, Maryland

April 1-6, 1979

\section{U.S. Department of Energy}

Assistant Secretary for Energy Technology

Division of Electric Energy Systems

Washington, D.C.

Under Contract No. C-77-C-01-5011 


\section{NOTICE}

This report was prepared as an account of work sponsored by the United States Government. Neither the United States nor the United States Department of Energy, nor any of their employees, makes any warranty, express or implied, or assumes any legal liability or responsibility for the accuracy, complateness, or usetulness of any information, apparatus, product, or process disclosed, or represents that its use would not infringe privately owned rights. Reference herein to any specific commercial product, process, or service by trade name, mark, manufacturer, or otherwise, does not necessarily constitute or imply its endorsement, recommendation, or favoring by the United States Government or any agency thereof. The views and opinions of authors expressed herein do not necessarily state or reflect those of the United States Govornment or any egency thereof. 


\section{ACKNOWLEDGEMENTS}

This research was conducted under contract $C-77-\mathrm{C}-01-5011$ from the U.S. Department of Energy. We would like to thank T. Trygar, L. Fink and K. Carlsen from the Department of Energy who have been monitoring this research and with whom we have had some useful discussions. We also had a valuable exchange of information with Bob Fischl and J. Wasley From Drexel Uriversity whom we thank. 


\section{PREFACE}

This preliminary report is subdivided into chapters which are essentially self-contained and could be read independently. The first two chapters provide the background and motivation for this research into security regions, as well as tying all the chapters together. Chapter 3 is a summary of the main results obtained and directions of research followed. In addition, this chapter describes the actual and proposed future extensions of this work. Chapters 4, 5, 6 and the Appendices provide a detailed description of the work carried out in the first two years of this contract. 
1.0 INTRODUCTION

1.1 Motivation and Background 1

1.2 System Security - Definitions and Objectives 3

1.3 System Security - Present Methods. 5

1.4 steady State System Security . 7

2.0 SECURITY REGIONS $\quad 12$

2.1 Motivation $\quad 12$

2.2 Definition of steady state Security Regions 15

- 2.2.1 Preliminary Remarks 15

-2.2.2 General Definitions in the Implicit Form 15

2.3 Rescarch objectives 20

3.0 PROGRESS SUMMARY . 21

3.1 Steady State Stability or Feasibility 21

3.2 Present and Planned Research in Steady State
Stability

3.3 Security Regions Imposed by Engineering

3.4 Present and Planned Research in security

Regions Imposed by Engineering Constraints 32

4.0 STEADY STATE STABILITY OR FEASIBILITY

4.1 Background 33

4.2 Closest Boundary Injection - Description and
Theory

4.3 Algorithms 58

4.4 Sufficient Feasibility set ? 85

4.5 Closest Boundary Injection with EqualityCon-
straints

$5.0 \frac{\text { CHARACTERIZATION OF THE SET OF SECURE SOLUTIONS IN }}{\text { THE VOLTAGE SPACE }} 100$

5.1 Introduction $\quad \cdot 100$

5.2 Security Constraints $\quad 104$

5.3 Auxiliary Inequalities 106

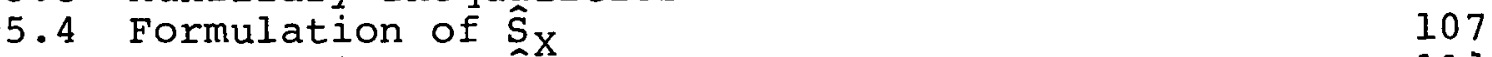

5.5 Formulation of $\hat{\mathrm{L}}_{\mathrm{X}}$ 
PAGE

5.6 Numerical Considerations . 115

5.7 Properties of $\hat{\mathrm{S}}_{\mathrm{X}}\left(\underline{\mathrm{z}}_{\dot{\mathrm{L}}}\right) \quad 117$

5.8 Examples 120

5.9 Conclusions . , 131

APPENDIX A-5

APPENDIX B-5

APPENDIX C-5 136

APPENDIX D-5 $\quad . \quad 138$

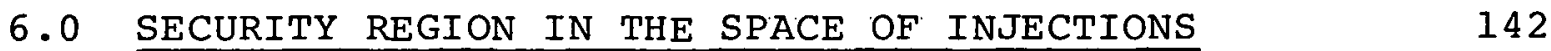

6.1 Motivation $\quad 142$

6.2 Transformation Formulae ... 143

6.3 Explicit Characterization of $S$

6.4 Filtering out the Redundant Constraints $\quad 159$

6.5 Other Formulations of the Fitting Problem 167

6.6 Present and Projected Works 17.2

$\begin{array}{ll}\text { APPENDIX A-6. } & 174\end{array}$

$\begin{array}{ll}\text { REFERENCES } & 184\end{array}$ 


\subsection{INTRODUCTION}

\subsection{Motivation and Background}

The purpose of this study is to investigate the concept of security regions in the context of power system security analysis. Power system security can be thought of as that characteristic of a system which guarantees the continuity and quality of service in spite of a number of potential disturbances or contingencies. These random events whinh scour with a finite probabilily include short circuits, line, transformer and generator outages; and load changes. The cause of their occurrence is often uncontrollable, being due partly to weather or other environmental effects, deterioration of equipment, and human error. A contingency may also be due to the failure of a neighbouring piece of equipment which then causes an overload, or to the incorrect operation or design of the power system. The result of a contingency can be relatively minor as with the successful clearance of a short-circuit which may merely lead to a flickering of the local voltage, or it can be catastrophic as in a major black-out. The cost of such an event is huge, both materially and in terms of social disruptions $[17,16]$ As long as the present structure of generating power at very large stations and transmitting this energy through a grid to the loads prevails, major and minor black-outs will continue and possibly intensify within the foreseeable future [19] . 
There exist many levels of effort for monitoring and controlling the security of a power system $[20,21]$ Thus, the system of relays and circuit breakers distributed throughout the network forms the most rapidly acting line of defense to prevent damage to equipment or the total loss of the power system. Voltage and speed regulators as well as controllable synchronous and static capacitors also serve to maintain voltages near their nominal levels and improve the system stability, both necessary conditions for the security of the network. In addition, because of the interconnected nature of power grids today, it becomes necessary when monitoring and controlling the effect of a contingency, to analyze large areas, such as whole companies, or pools of companies. The real time monitoring and the control of the security of large interconnect power systems is an activity which is being or will be incorporated in many system or pool dispatch centers $[21,22,23,24]$.

The philosophy behind centrally located dispatch functions monitoring and controlling the security of the system, is based on the following factors:

(1) Security can be incorporated into the power system at the planning level only up to a limited extent. The reason being that planning studies are based on load and network predictions which may not materialize.

(2) Although the addition of extra transmission and generation capacity can substantially improve the system security, their cost and the many environmental and right-of-way constraints, limit this approach. 
(3) The cost of installing and operating a dispatch center and all its associated computing, communication, and measuring equipment is small compared to the cost of a new power plant or an EHV transmission line.

(4) The increased demand and complexity of future power systems makes it progressively more difficult to monitor and control system security without the help of powerful computers.

(5) More systematic approaches and more complex system models will be needed to "guide" the operating point through constraints imposed by the systems of today and those to come.

In addition to the above factors based mainly on operational considerations, further motivation for investigating the problem of system security. can be found at the planning level. Here, expansion decisions of, a complex nature have to be made which are compatible with the security limitations and which account for the uncertainty associated with long-term predictions.

\subsection{Syetcm Eecurity-Definitions and ubjectives}

Power system security is that property of a power system which defines its ability to operate satisfactorily often the occurrence of any one of a number of postulated probable component failures or contingencies. Two desirable properties from the security point of view are that: 
(I) The transients following a contingency are stable and damp-out to a steady state without in the process causing another failure (e.g. that a breaker be tripped by the transient).

(2) The resulting steady state operating point does not violate any steady state constraints, (e.g. a thermal over-load, or an over-voltage).

The various levels and degrees of security have been well defined and documented $[25,26]$.

A system is secure if all of the postulated contingencies stabilize to a steady state without creating overloads or violating constraints.

A system is vulnerable or in the alert state if one or more of the contingencies would result in either instability or a steady state constraint violation. The system can still be operäted in thls vuliserable statc, and will behave normally as long as the above-mentioned contingencies do not occur:

The purpose of security monitoring is to test the potential effect of the postulated contingencies and to detect and identify those to which the system is vulnerable. This task be carried out in real time at the operational level, accounting for the hourly variations of the system structure and load. In this case very rapid and efficient schemes are therefore essential.

At the planning stage security monitoring may also be carried out based on the predicted load and network 
expansion plans. The uncertainty associated with these predictions makes it difficult, however, to anticipate all probably contingencies at the planning stage. The most critical operating condition is the emergency state. Here a contingency has occurred resulting in an unstable system or in a new steady state which violates some of the security constraints. An unstable situation is the more serious, and emergency measures must be implemented within seconds. These actions may involve disengaging some generation from the system or shedding load. A steady state violation can be corrected by similar means, but usually more time is available to execute this action. These security control measures (also known as corrective rescheduling) can be executed after the on-set of the contingency, if time permits, and are then termed emergency control, or they can be implemented as preventive control measures. In this approach the system is modified, when a vulnerable contingency is detected, and brought. hark into a statc not vulnerable to the most probable contingencies. Preventive control is preferable to emergency control, but its implementation may not. always be possible because of the system constraints, or because of the cost of rescheduling generation to a secure state.

1.3 System Security - Present Methods

Because of the complexity and size of power systems today, the study of their security is based primarily on numerical simulations using the digital computer. The 
behaviour of a power system is thus usually analyzed by running a large number of simulations for the various types of normal and abnormal expected operating conditions. The conditions typically simulated are:

1. Electromagnetic transients

2. Short and open circuits.

3. Sinusoidal steady state or load flow

4. Electromechanical transients or transient stability

The first program serves to study the effect of very fast transients and overvoltages due to disturbances such as lightning strikes and switching operations. Insulation levels are set with the help of these simulations.

Short circuit analysis is concerned with the effect of a disturbance such as a short circuit or an open circuit. Relay settings to protect the system equipment against overloads are made with the help of this program.

The load flow program analyzes the sinusoidal steady state behaviour of a power system, particularly the interaction among real and reactive power and voltage magnitudes. The normal operation of a power system must be done satisfying certain inequality constraints (security limits) on these variables.

Finally, the transient stability program is concerned with transients of the electromechanical type, slower but more serious than electromagnetic transients. These oscillations can cause the system to break up into groups of generators out of synchronism with each 
other, a condition which cannot be sustained for more than a fraction of a second before a blackout results.

Such programs will always play an important role in planning and operating power systems as they provide the user with quantitative data as well as a "feel" of the system behaviour. Considerable amount of money and effort has been put into making these programs computationally fast, and in reducing their storage requirements. Such, research and development is an important continuing effort $[27,28]$.

\subsection{Steady state system security}

This study has concentrated primarily on the security of power systems in sinusoidal steady state. This is the normal mode of operation of a power system, and it is at this stage that early detection and correction of vulnerable conditions can be most effective.

The basic mathematical model used to describe the system in sinusoidal steady state is the set of load flow equations [27] denoted by

$$
\underline{z}=\underline{F} \quad(\underline{x})
$$

where $\underline{z}$ is the vector of nodal injections while $\underline{x}$ is the vector of components of the complex network node voltages. The function $\underline{F}$ is a non-linear function of the variables $\underline{x}$ whose form is defined by the topology and the parameters of the power network. 
The nodal injections $\underline{z}$ can be divided into two. parts; i.e.,

$$
\underline{z}=\left[\begin{array}{l}
\underline{u} \\
\underline{z}_{L}
\end{array}\right]
$$

The vector $\underline{z}_{L}$ represents the vector of injections corresponding to the load or $P Q$ buses $i . e$. those buses where the real and imaginary power demand is fixed by the consumer. The vector $\underline{u}$ represents those injections which are controllable, and can be manipulated by the operator to satisfy security and economic objectives. Its components are usually the real power generated at the generation buses (except the slack or swing bus), and the voltage magnitudes of all such buses.

By specifying the vector $z$, equation (1) may be solved via a load flow numerical scheme [27] giving the corresponding state $\underline{x}$. Any other dependent system variable, $y$, can then be computed through an explicit function of $\underline{x}$,

$$
y=G(\underline{x})
$$

From the security point of view the dependent system variables of interest are:

1. Load bus voltage magnitudes

2. Generation bus reactive generations

3. Slack bus real generation

4. Magnitude of the Iine current flows 
These variables are all limited by upper and lower bounds defined by technical or performance specifications, and the system should be operated in such a way that these variables do not violate any of these security limits. The alternative could be as serious as a major black-out, a damaged piece of equipment, or a deterioration of the service (e.g. a low load voltage). The security limits can be expressed as

$$
\mathrm{y}^{\mathrm{m}} \leq \mathrm{y} \leq \mathrm{y}^{\mathrm{M}}
$$

where $y^{M}$ and $y^{m}$ represent respectively the veston of upper and lower bounds of the elements of $y$.

$$
\text { For similar reasons it also follows that the }
$$

controllable variables, $\underline{u}$, are restricted through

$$
\underline{u}^{\mathrm{m}} \leq \underline{\mathrm{u}} \leq \underline{u}^{\mathrm{M}}
$$

Since the loads are limited as well, one can say that in general the injections, $\underline{z}$, satisfy,

$$
\underline{z}^{m} \leq \underline{z} \leq \underline{z}^{M}
$$

All the above mentioned inequalities are denoted as the security constraints of the system.

The problem of steady state security monitoring can be formulated as follows:

Given a network structure (i.e. the function F) and. the injections $\mathrm{Z}$ determine whether the system equalities and inequalities are satisfied, i.e. verify the validity of the relations (1), (4) and (6). This check is carried out by solving (1) via a load flow [27] for $x$ and testing 
(4) and (6). This procedure is repeated for each contingency in the probable contingency list. Although efficient load flow algorithms exist for this purpose [29, 30 ] the basic security monitoring approach is essentially a repeated application of a load flow for each contingency. The security control problem is considerably more complex: Given that a certain contingency violates security, the objective is to redefine the controllable vector, $\underline{u}$, or the system structure, such that:

(1) The system is no longer vulnerable to the contingency.

(2) The new steady state does not violate any security constraints.

(3) The new steady state is not vulnerable to the remaining contingencies.

(4) If possible, a crilerion meaouring the oppration cost in dollars or the level of environmental contamination is minimized.

Under emergency control the satisfaction of the load equality constraints is not always possible, and load shedding or voltage decreases (brown-out) become necessary. The objective here also changes to, for example, minimizing the amount of load dropped. The primary tool of a. systematic nature, available for security control is the optimum load flow $[28,31]$ This program is at present not well suited for real time implementation, however its implementation in either the 
general or some approximate form is planned by most major utilities for the near future. This objective is in recognition of the fact that systematic methods of controlling the security of the system will be essential to minimize all types of service interruptions or deteriorations. 


\subsection{SECURITY REGIONS}

\subsection{Motivation}

The present methodology for monitoring and controlling steady-state systems security is based on the repeated applications of two numerical algorithms: the load flow and the optimal load flow. This is a methodology which generates one numerical answer to each monitoring or control problem. Although these methods are very effective when analyzing one case at a time, they have certain drawbacks when one becomes interested in the system's security for a range of operating conditions and for a large number of network structures, as is often the case. Thus, if the system injections, $\underline{z}$, vary, this system security approach requires the recomputation, for each change in $\underline{z}$, of as many load flow as there are contingencies, and the verffleation, fur edill lual flow solution, of all the inequality constraints on the dependent variables. Since the possible range of variations of $\underline{z}$ is very large, the number of load flow computations and inequality tests is a task which can occupy a large computer full time. This monitoring effort is compounded by the fact that the network structure also may change with time.

The use of an optimal control scheme in a real time mode is even more difficult to implement. Thus, if a control vector $u_{i}$ is found which corrects the vulnerability of the system to a given contingency, there is no guarantee that the implementation of such a control will not then 
make the system vulnerable to the remaining contingencies. There is no simple way of modifying the optimum load flow to consider all contingencies at once without augmenting the dimensionality of the problem by a factor equal to the number of contingencies. This becomes then a task for which no practical solution exists at present. One exception is the case of emergency control where only the constraints corresponding to the violating contingency nepr to bo trcated. Here the policy is to find any secure solution to the emergency at hand, regardless of the cost and of whether the new solution is vulnerable to other potential outages.

The above remarks motivate the following general directions of research:

(1) Do there exist simple, fast, and reliable schemes for monitoring the security of a power system for a large set of postulated outages and over a wide range uf Injections which do not require the explicit evaluation of one load flow for each contingency and for each variation in $\underline{z}$ ?

(2) Does there exist a similar scheme for controlling the system's security?

(3) Can analytic results be obtained describing the general nature of a secure power system?

One possible direction to follow in order to implement these general aims is to develop faster, more efficient load flow and optimal load flow schemes. This is one 
approach which we have not pursued, but which may still lead to improvements from the algorithmic point of view or through special purpose computers [32]. Although this dependence on computer-based numerical simulations will inevitably continue, this approach has the drawback that it does not easily contribute to our knowledge of the general analytic properties of a secure system. This is an aspect which is theoretically and practically important in the area of security analysis. The analytic or semianalytic approach essentially establishes properties of a range or region of the variables or parameters characterizing a secure power system. Thus, in contrast with the numerical approach which generates one solution at a time; here we may establish, for example, that all secure systems must satisfy certain simple to verify, necessary curlalions on the injertions. Another type of analytic statement may be sufficient in nature, declaring that if the system's injections satisfy certain simple conditions the security will be guaranteed.

The potential benefits of the analytic approach based on security regions include the development of systematic security monitoring and control schemes, simple, cheap and efficient, which will put these tasks within the reach of most utilities. In addition, important theoretical and practical knowledge and insight of a basic nature can be developed via the analytic approach. 


\subsection{Definition of Steady state Security Regions}

\subsubsection{Preliminary Remarks}

In this study we distinguish between a security region in the space of voltages and in the space of injections. Although the second type is much more useful for security analysis purposes, the security region. in the space of voltages adds important insight to the overall concept, and as will be seen, also leads to practical security algorithms. We also distinguish between the explicit and implicit representation of a security region. The implicit description is simply a statement of the conventional security analysis approach based on the load flow and the subsequent verification of the inequality constraints, or on the optimal load flow. Only very general properties about the nature of the set can be established with its implicit characterization. The explicit description, on the other hand, describes the security set or part of it, through some explicit analytic expressions in the system variables, which are easily manipulated or analyzed for both security monitoring and control.

2.2.2 General Definitions in the Implicit Form

In this study we have treated two general forms of security regions:

(1) The set of vectors of complex bu's voltages for which', for a given network structure, all security inequalities are satisfied. 'Thls set 'is called the security region in the space of voltages and is given the symbol $s_{x}$. 
(2) The set of vectors of bus injections for which, for a given network structure, all security inequalities are satisfied. This set is called the security region in the space of injections, and is given the symbol $\mathrm{S}_{\mathrm{z}}$ :

A number of important regions related to the two principal ones are also investigated:

(a) The set $S_{x}\left(\underline{z}_{L}\right)$. The set of all bus voltages which satisfy all inequality constraints and which also satisfy certain equality constraints (e.g. some specified. complex loads, $\underline{z}_{L}$ '.

of additional interest in this study is the characterization of those security limitations imposed on the injections because of physical realizeability or feasibility restrictions, due to the network structure and the non-linearity of the load flow equations $[1,2,16]$ These constraints are also known in the litorature as steady state stability limits and their satisfaction is necessary for the transient stability of the system. (b) The set $R_{z}$ is the set of all injections for which, for a given network, a physically realizeable or feasible steady state is possible.

The set $R_{z}$ is clearly part of $S_{z}$ i.e. a vector of injections $\underline{z}$ must first be feasible, and then its steady state solution must satisfy the security inequalities on the dependent variables. On the other hand, an unfeasible $\underline{z}$ will result in a transiently unstable system which clearly violates any steady state 
inequalities. The set $R_{z}$ then imposes additional Iimitations on the injections which must be includealas part of the overall security constraints, i.e. the set of $\mathrm{S}_{z}$. The questions to which we address ourselves in this study is how to explicitly characterize these feasibility constraints and incorporate them into $\mathrm{S}_{\mathrm{z}}$. We also look at the effect on the security regions of a list of probably network contingencies. The set of injections for which none of the list of probable contingencies would result in a security violation is denoted the vulnerability region.

Finally, emergency sets can be defined describing those injections which would result in the correction of an emergency state. Whereas a vulnerability set will be "small" or empty (i.e. no solution may exist for which the system is not vulnerable to some outage), the emergency set is a "larger" one making it easier to return an insecure emergency state into this set, thereby correcting the insecure situation.

The mathematical definition of the above-mentioned sets is as follows:

(1) Security Region in the Space of Voltages $S_{x}=\left\{\underline{x} / y^{m} \leq \underline{G}(\underline{x}) \leq y^{M}, \underline{z}^{m} \leq \underline{F}(x) \leq \underline{z}^{M}\right\}$

(2) Security Region in the Space of Injections $S_{z}=\left\{\underline{z} / \underline{z}=\underline{F}(\underline{x}), \underline{x} \varepsilon S_{x}\right\}$

It. can be seen that $S_{z}$ is the mapping of the set $S_{x}$ through the load flow equations $\underline{z}=\underline{F}(x)$ into the space of injections. 
$\mathrm{S}_{\mathrm{x}}$

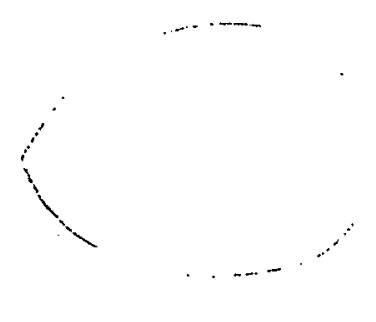

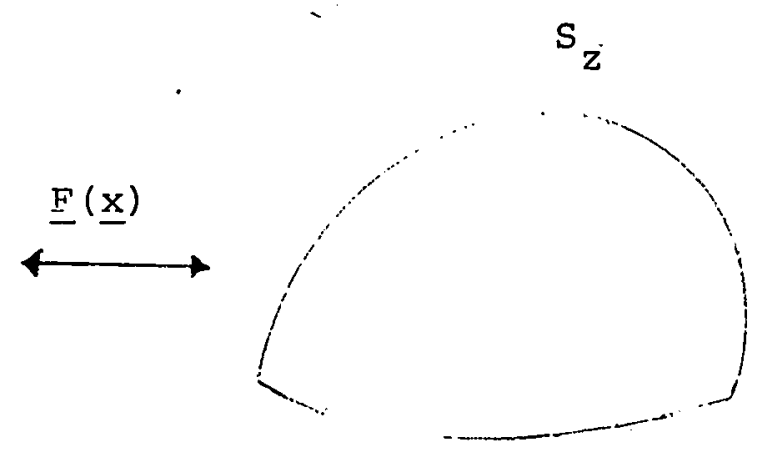

The set $S_{x}$ is characterized by the intersection of the subsets

$$
y_{i}^{m} \leq G_{i}(\underline{x}) \leq y_{i}^{M}
$$

and

$$
z_{j}^{m} \leq F_{j}(\underline{x}) \leq z_{j}^{M}
$$

for all inequalities. An important observation is that these subsets are described by explicit analytic functions of $x$. In fact, if $x$ represent.s the real and imaginary parts of the complex bus voltages, then the functions $G_{j}$ and $F_{j}$ are quadratic in $x$. Such functions are susceptible to analytical studies which lead to the extraction of general properties of the set of secure solutions. (See next section for some examples).

By defining $S_{x}$ first through $(\mathfrak{i})$ and the defining $S_{z}$ as the mapping of $S_{x}$ through the singled valued real function $\mathrm{F}$, we guarantee that all the elements of $\mathrm{S}_{z}$ will be feasible, thus implicitly accounting for the feasibility set, $R_{z}$ As will be seen later on, it is not practical to analyze $\mathrm{S}_{z}$ in this implicit manner and an explicit analysis of $R_{z}$ and $S_{z}$ will be needed. 
The set $S_{x}$ then implies that the power network will be secure to the contingency corresponding to the asilmed network structure if and only if $\underline{x} \varepsilon S_{x}$. Alternatively, the same condition will be satisfied if and only if $\underline{z} \varepsilon S_{z}$. Although the set $S_{z}$ is not explicitly described (in contrast to $s_{x}$ ) in terms of $\underline{z}$, this set is more useful than $S_{x}$ since the former defines security directly in terms of the specified or controllable injections. It is these quantities which are manipulated in order to control security.

The vulnerability region is another useful concept. If we assume that the probable next contingency list contains $K$ different contingencies and a corresponding number of network structures, then for each contingency $\mathrm{k}=1,2, \ldots, \mathrm{k}$, a security set $\mathrm{s}_{\mathrm{z}}^{\mathrm{k}}$ can be defined through with different $\underline{G}^{k}$ and $\underline{F}^{k}$. The vulnerability set is defined by

$$
\prod_{k-1}^{\mathrm{K}} \mathrm{s}_{\mathrm{z}}^{\mathrm{k}}=\text { intersection of all } \mathrm{s}_{\mathrm{z}}^{\mathrm{k}}
$$

Thus an injection vector $\underline{z}$ is vulnerable to one or more of the contingencies if and only if $\underline{z} \bigcap_{\mathrm{k}=1}^{\mathrm{K}} \mathrm{s}_{\mathrm{z}}^{\mathrm{k}}$. Equivalently a system will not he vulnerable to any of the postulated outages if and only if $\underline{z}$ belonge to the vulnerability set. The equivalent vulnerability set in the space of voltages if defined by

$$
\left\{\underline{x}_{k}, k=1, \ldots, k / \underline{x}_{k} \varepsilon s_{x}^{k} k=1, \ldots, k\right\} \text {. }
$$


i.e. the vector of voltages corresponding to each contingency, $\underline{x}_{k^{\prime}}$ must belong to the corresponding security set $S_{x}^{k^{\prime}}:$ In the space of voltages, vulnerability is therefore defined in a higher dimensional space (by a factor of $\mathrm{K}$ ), with its corresponding increase in complexity. In contrast, the dimension of the vulnerability set in the space of injections remains the same as that of the security set.

\subsection{Research Objectives}

The general research objectives of this study are

as follows:

(a) To precisely define the concept of steady state security regions.

(b) To determine general properties of these regions.

(c) To characterize their properties through simple, explicit, analytic relations. The basic aims of this phase are:

(i) To be able to rapidly and reliably find from this set operating points (either $\mathrm{x}$ or $\mathrm{z}$ ) for a given network which will guarantee the satisfaction of its security constraints.

(ii) To be able to simply generate from the vulnerability set operating points which will not be vulnerable to any of a probable list of contingencies. 
(iii) To use the security or vulnerability regions to rapidly and reliably monitor the system's security.

(d) To investigate the potential applications of these regions to power system planning and operation.

(e) To generate basic results on the question of steady state power system security.

\subsection{PROGRESS SUMMARY}

In this section we summarize the progress made thus far in this investigation. Subsequent sections and the appendires describe these results in more detail.

The two main phases of this study have been:

(a) Steady State Stability or Feasibility Region.

(b) Security Regions Imposed by Engineering Constraints.

\subsection{Steady state Stability or Feasibility}

The first phase of this research has concentrated on the analysis of the feasibility region, $R_{z}$, characterizing the set of all injections which, for a given network structure, result in a physically realizable or feasible loadflow. This is equivalent to the set of injections, $\underline{z}$, for which a real

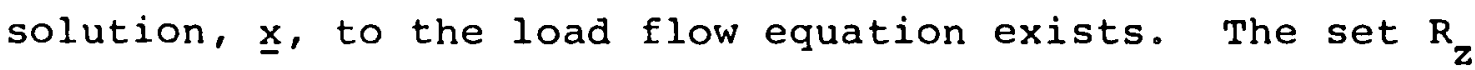
is defined by

$$
\mathrm{R}_{\mathrm{z}}=\left\{\underline{\boldsymbol{z}} / \underline{\mathbf{z}}=\underline{\mathrm{F}}(\mathrm{x}), \underline{\mathrm{x}} \varepsilon \mathrm{R}^{\mathrm{n}}\right\}
$$

Here $R^{n}$ represents the $n$ dimensional Euclidian space where $n$ is the dimension of $x$ (and of $n$ ). Thus far the following principal results have been obtained: 
(1) General properties about the nature of $R_{z}$ have been established showing that $R_{z}$ is a convex cone lying on or above a set of supporting hyperplanes. Each supporting hyperplane is tangent to a ray on the boundary of $R_{z}$ and defines the feasibility condition

$$
\alpha^{T} z \geq 0
$$

where $\underline{\alpha}$ is the constant vector perpendicular to the supporting plane. This is a necessary condition for feasibility, i.e. $\underline{z} \varepsilon R_{z}$ only if $\underline{\alpha}^{T} \underline{z} \geq 0$ is satisfied.

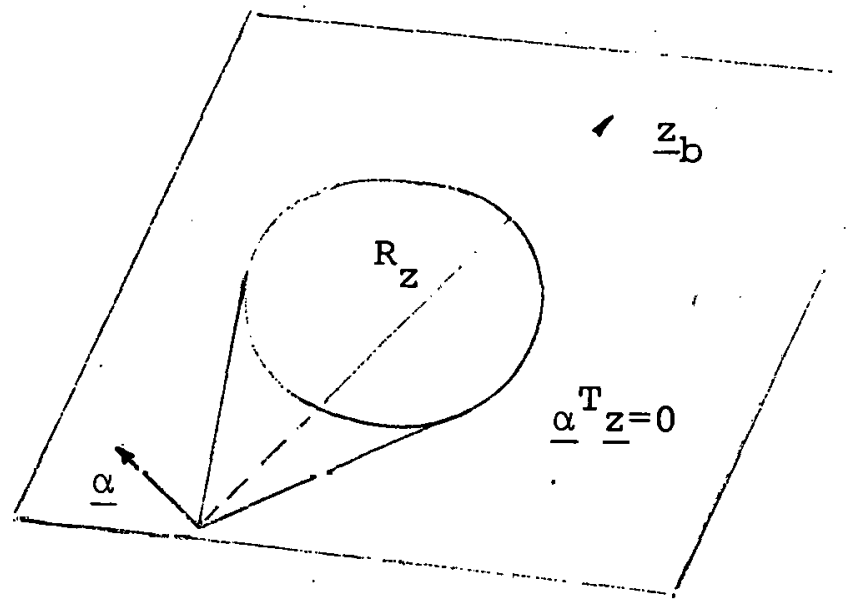

(2) The feasibility conditions represent simple-to-verify necessary conditions for $z$ to belong to $R_{z}$. Very efficient schemes have been developed for finding arbitrary feasibility planes, or planes "close" to a normal region of operation.

(3) One of the critical questions in steady state stability, which is answered here, is as follows:

Can a normal operating point, $\underline{\boldsymbol{z}}$, undergo a reasonable • change within its expected range of variation and in so doing become unfeasible? 
This is not an unreasonable occurrence if we consider, for example, a'situation where the system load increases without a corresponding increase in voltage. Unfeasible conditions are even more conceivable in the event of major transmission line outages during heavy-load periods combined with low generation due to generator maintenance. Unfeasibility can also be aided by the failure of voltage-boosting equipment such as synchronous or static capacitors.

Unfeasibility, i.e. the boundary of the feasibility region, can be reached in an infinity of ways, oome more likely than others. What we have resolved here is the computation of the "shortest" path from an arbitrary operating point, $\underline{z}_{g}$, to the boundary of $R_{z}$. The term, "shortest", implies that we find a ray on the boundary of $R_{z}, \underline{z}_{b}$, such that the angle between $\underline{z}_{\mathrm{g}}$ and $\underline{z}_{\mathrm{b}}$ is minimum over the entire boundary of $R_{z}$. The shortest path in the Euclidian sense from $\underline{z}_{g}$ to the boundary of $R_{z}$ is then the vector projecting $\underline{z}_{g}$ onto $\underline{z}_{\mathrm{b}}$.

As will bc clarified in section 4 , this result has various consequences:

(i) The feasibility condition corresponding to the closest boundary point, $\underline{\alpha}^{T} \underline{z} \geqslant 0$, is both necessary and sufficient for $\underline{z}_{\mathrm{g}}$ to be feasible. From this, one can therefore independently of a load flow numerical scheme, determine whether a given operating point, $\underline{\mathbf{z}} \mathrm{g}$ ' is feasible or not. If $\underline{\mathbf{z}} \mathrm{g}$ is not feasible then $\underline{\alpha}^{T} \underline{z} \geqslant 0$ can serve as an additional explicit constraint to be considered together with the engineering constraints on $\underline{z}$. 
ii) The concept of steady state stability can be quantified for a general power network. Associated with an arbitrary $\underline{\mathbf{z}}_{\mathrm{g}}$, we can define and compute a feasibility or steady state stability margin, SM, defined by

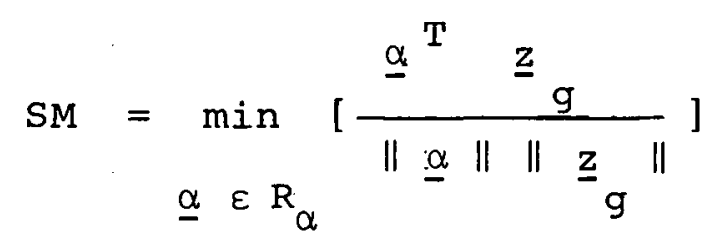

The quantity between brackets represents the cosine of the angle between $\underline{\alpha}$ and $\underline{z}_{g}$. The norm $\|\cdot\|$ is the Euclidian norm. The set $R_{x}$ contains only the vectors $\underline{\alpha}$ defining the support hyperplanes of $R_{z}$. The particular $\underline{\alpha}$ defining $S M$ is the one which minimizes the cosine of the angle between $\underline{\alpha}$ and $\underline{z}_{g}$. This is equivalent to minimizing the angle between $\underline{z}_{g}$ and the rays on the boundary of $R_{z}$. It therefore follows that if $\mathbf{z}_{\mathrm{g}}$ is feasible then $\mathrm{SM}>0$, and if $\underline{z}_{\mathrm{g}}$ is unfeasible then $\mathrm{SM}>0$. Since SM lies between -1 and +1 we can associate with this range of numbers a measure of unfeasibility or of feasibility. For most practical purposes, an operating point with a value of $S M=0.7 \mathrm{will}$ be "more" stable in the steady state sense then one whose $\mathrm{SM}=0.5$.

If an operating point $\underline{z}_{g}$ is unfeasible, i.e. SM is negative, then this result also describes the shortest path from $\underline{z}_{g}$ to $R_{z}$, i.e. it tells/what is the smallest (in the Euclidian sense) variation in $\underline{z}_{g}$ which will bring us back to a feasible operating point. Furthermore, because of the stability measure SM, we can also easily determine the minimum change in $\underline{z}_{g}$ which will result in a minimum desired stability margin. 
iii) The concept as well, as the algorithm for computing the closest point in $R_{z}$ with a specified stability margin has also been extended to the more general case where the variation in $\underline{\mathbf{z}}$ is restricted to a certain sub-space. For instance, it is more realistic to assume that only the components of $\underline{z}_{g}$ corresponding to certain injections such as loads and the generation can change, or that only the generator voltage magnitudes change. This may give a more realistic view of where the closest boundary point lies than if we allow all injcctiong to valy. The clusese boundary point with constraints is very useful when corrective re-scheduling is necessary to return $\underline{\mathrm{g}}_{\mathrm{g}}$ to the feasibility region or to achieve a desired stability margin in the 'controllable variables only.

iv) The concept of the closest boundary point also produces; as a by-product, a simple-to-verify sufficient condition for feasibility in the neighbourhood of a feasible $\underline{z}_{g} \cdot$ Geometrically this set is a circular cone with $\underline{z}_{g}$ as its vertex and tangent along $\underline{z}_{b}$ to the support hyperplane corresponding to this boundary ray. Such a set is defined by

$$
\underline{\alpha}^{\mathrm{T}} \quad \underline{\mathrm{z}}_{\mathrm{g}} \geqslant\left(1-S M^{2}\right)^{\frac{1}{2}}\|\underline{\mathrm{z}}\|\left\|\underline{\mathrm{z}}_{\mathrm{g}}\right\|
$$

Graphically, this conccpt is described in Fig. 4.11 for a top crosssection of $R_{z}$ in 3 dimensions, 
(v) Extensive numerical tests have been made to implement these concepts. Very efficient algorithms have been developed and applied to various sample power networks.

The details of these ideas are presented in Chapter 4 and the Appendices.

3.2 Present and Planned Research in Steady state Stability We are presently studying or propose to investigate the following additional topics relative to steady state stability or feasibility: .

(a) Improved numerical methods for the computation of the closest boundary point, the corresponding $\underline{\alpha}, S M$, and the sufficient cone. A large decrease in computational effort based on the inverse power method [12] a technique in eigenvalue analysis, has been recently obtained and is being tested further.

(b) Methods of "maximizing the size" of the sufficient cones to include as much volume of $\mathrm{R}_{\mathrm{z}}$ as possible.

(c) The intersection of the feasibility region with these constraints imposed by engineering limitations will be further investigated and coupled to the second theme of this investigation. 
(d) The application and test of these concepts to some typical power networks are being pursued. Here we wish to determine the severity of the feasibility constraints, the nature of the computational problems, the effect of multiple line outages on the feasibility region, the effect of very long EHV lines, the relationship to transient stability, and the effect on feasibility of network devices such as shunt reactors and capacitors, tap-changing transformers, and DC transmission lines.

\subsection{Security Regions Imposed by Engineering Constraints -}

This phase of the investigation proceeded along two main lines. The first was an analysis of $\mathrm{S}_{\tilde{x}}$, the security region in the space of voltages, followed by an analysis of $\mathrm{S}_{z}$, the equivalent region in the space of injections. It turns out that certain properties can be best determined in $\mathrm{S}_{\mathrm{X}}$, while yet other computations are best carried out in the space of injections. A good understanding of the two. regions is in any wase necessary and the properties of both have been thoroughly analyzed.

In Section 5, a detailed description is given of a technique whose basic approach is to define a set $\hat{\hat{s}}_{\mathrm{x}}$ (eimpler to describe than $S_{x}$ ) which contains the security set. $\hat{s}_{x}$, thus

$$
\hat{s}_{x} \supset s_{x}
$$

The set $\hat{S}_{x}$ is defined by a finite number of linear inequalities in $\underline{x}$ dependent on the network structure and on the given security inequalities. The method exploits the special quasilinear load flow equations in the surrent form. The practical 
and original aspect of this part of the investigation lies in the ability to manipulate the non-linear load flow equalities and the security inequalities and replace them with a finite number of linear inequalities in $\underline{x}$ which are still necessary conditions for security. This procedure involves some unique manipulations of these relations.

The objective of this phase was to investigate the nature of the non-linear security set $S_{x}$ through the linear set, $\hat{s}_{\mathrm{x}}$. The latter is susceptible to linear analysis techniques such as linear programming (LP), a very powerful analysis tool. The fact that $\hat{S}_{x}$ contains $S_{X}$, and the fact that $\hat{\mathrm{s}}_{\mathrm{x}}$ is simply parameterized by the network parameters provides us, through LP, with a simple tool to determine whether $\hat{\mathrm{S}}_{\mathrm{x}}$ is empty (i.e. if no feasible solution is found by $L P)$. The affirmative case therefore implies that $S_{X}$ is also empty, that is, that no secure solution can exist under the specified equalities and inequalities. This is a critical condition which is difficult to detect systematically. The other feature which makes this result interesting from the practical point of view is that $\hat{\mathrm{s}}_{\mathrm{x}}$ is a"close" approximätion to $\mathrm{s}_{\mathrm{x}}$. This means that $\hat{\mathrm{s}}_{\mathrm{x}}$ surrounds $\mathrm{s}_{\mathrm{x}^{\prime}}$, but not in an overly conservative manner (see Section 5 for details and. examples). No rigorous proof for this statement has been found except for experimental runs which tend to confirm it. One of the implications of this closeness is that when $s_{x}$ is non-empty the use of an LP routine maximizing arbitary criteria over $\widehat{S}_{x}$ will yield a value of. $x$ which is not "far" from $S_{x}$ " i.e. which does not violate many inequalities; and such that 
the equality violations are "small". From this value a simple technique is then used (see Section 5), which finds an arbitrary voltage vector belonging to $\mathrm{S}_{\mathrm{x}}$. ' Such a vector can then be used as a feasible starting point for an optimal load flow scheme. This computation of an arbitrary secure $\underline{x}$ is a result for which no systematic equivalent exists at present.

In Section 6 , we describe the efforts made to characterize $\mathrm{S}_{\mathbf{z}}$. As discussed earlier, the description of the security region in the space of corliullable parameters leads to more practical security monitoring and control schemes.

One of the first steps taken was a detailed analysis of the security regions of a two bus system (see section 6 for details and examples). This example illustrates graphically the concepts and the exact shape of this region, demonstrating the non-linear and non-convex nature of $\mathrm{S}_{\mathrm{x}}$ and $\mathrm{S}_{\mathrm{z}}$. Another important feature which is clearly illustrated is that many of the constraints defining the security regions are redundant. The identiflcation of the active or of the redundant constraints is an important result which we have also investigated.

In order to explicitly characterize the set $\mathrm{S}_{z}$ for a general power network, we first studied means of describing the dependent network variables by an explicit analytic function of $\underline{z}$. (See Section 6 and Appendix A-6). The simplest (but nevertheless accurate) of such formulae were of the form,

$$
y \simeq \underline{\beta}^{T} \underline{z}
$$

or

$$
y \simeq \underline{\beta}^{T} \underline{z}+\underline{z}^{T} \underline{C} \underline{z}
$$


where $y$ is a constrained dependent variable, $\underline{B}$ is a constant vector, and $\underline{C}$ is a constant real symmetric matrix. If we denote such explicit approximations by $y(\underline{z})$, the security set $S_{z}$ could be expressed by

$$
s_{z}=\left\{\underline{z} / y_{i}^{M} \leqslant y(\underline{z}) \leqslant y_{i}^{M}\right\}
$$

The explicit description of $\mathrm{S}_{\mathrm{z}}$ using the linear approximation formulae permits the application of techniques such as linear programming directly in the space of injections to solve the security control problem. The emergency problem can also be solved by a convex programming technique whose objective is to return the injection vector to the secure region with a minimum of load shedaing, or along the shortest possible path. Although $\mathrm{S}_{z}$ described by linear approximation. formulae can be subjected to many analysis techniques, its relative inaccuracy makes $1 t$ necessary to rccort to quarratic approximations. Here, analytic properties of quadratic formulae are also exploited to characterize $\mathrm{S}_{\mathrm{z}}$.

In spite of the simplifications achieved by explicitly expressing $\mathrm{S}_{\mathrm{z}}$ as the intersection of a finite number of linear or quadratic hypersurfaces, this characterization by itself is still not well suited for very rapid security checks or security corrections. The approach which we have followed and are still pursuing exploits the fact that the normal trajectory of the injection vector does not vary much from day to day. We need to characterize therefore only parts of $\mathrm{S}_{\mathrm{z}}$ around the injection trajectory. This has been done via sets which are sufficient but not necessary for security, 
and are described by hyperellipsoids whose centers are on the expécted trajectory.

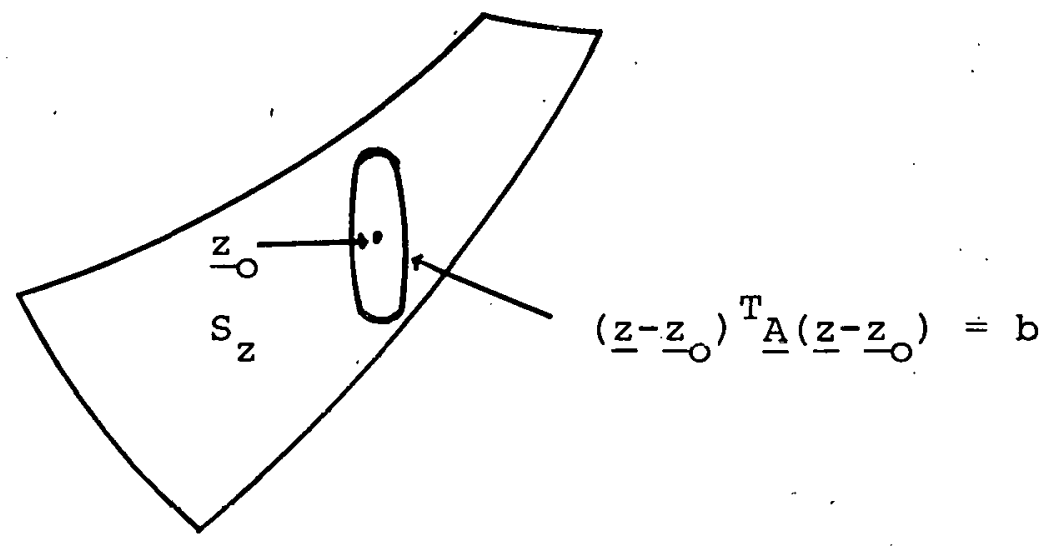

Various such ellipsoids can be chosen which completely enclose the prediotcd injecllun trajectory. Their centers lie on the trajectory of $\underline{z}$, while the orientation of the axes of the ellipsoid can be chosen so that the principal axis points in the direction of movement of $\underline{z}$. This determines the choice

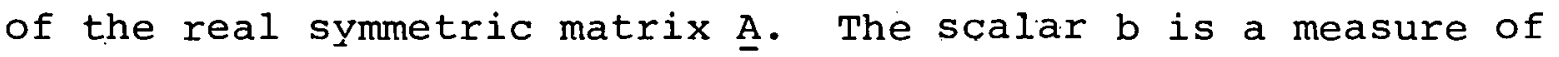
the "size" of the ellipse, and it defines the largest sudh ellipse centered at $\underline{z}_{0}$ within the set $S_{z} \cdot$ Since the boundary of $S_{z}$ can be explicitly characterized by analytic functions, the maximization of the ellipsoid with respect to all boundaries of $\mathrm{s}_{z}$ can be systematically carried out. The minimum of these maxima defines $b$.

The idea behind this technique is that although the computation effort of the sufficient cllipsoids is relatively high, this work can be done off-line: Onc aufficient ell1psoid can be described by the symmetric matrix A (usually diagonal)!, the center of the ellipse $z_{0}$, and its size b. The test for membership in the ellipse and hence in $s_{z}$ is simply to vorify whether, for a given $z$, the condition, 


$$
\left.\left(\underline{z}-\underline{z}_{0}\right)^{T} \stackrel{A}{\underline{z}}-\underline{z}_{0}\right) \leqslant b
$$

is satisfied. This is a great simplification over the hundreds of load flow computations and inequality checks that are normally needed in security monitoring. The violation of ( 1 ) does not necessarily imply insecurity, but simply that the expected trajectory has deviated abnormally from its normal path. In such a case a standard security monitoring scheme could be used instead. However, since the injection path is well predictable such deviations may not occur very often. Various techniques are also suggested in section 6 to execute fast preventive or emergency control actions based on the concept of sufficient security sets.

3.4 Present and Planned Research in Security Regions Imposed by Engineering Constraints

We are investigating or propose to investigate the following topics relative to this phase of the project:

(a) Further tests of the accuracy of the approximating formulae relating the constrained dependent variables to the independent. injections.

(b) The development of emergency control schemes with the explicit security inequalities in the injections as constraints. (c) Further analysis and development of algorithms to compute sufficient security sets.

(d) The application of sufficient sets to carry out security monitoring and control in sample systems.

(e) Maximization of the "size" of the sufficient security sets.

(f) Incorporation of steady state stability constraints, if active, in the security region. 


\subsection{STEADY STATE STABILITY OR FEASIBILITY}

\subsection{Background}

Some of the background material presented in this section appears in $[1,2,16]$. The question treated in this phase of the project concerns the limitations imposed by a power network on the specified nodal power and voltage quantities if the corresponding load flow is to be physically realizeable or feasible. These steady state stability limitations are well understood for the simple case of one generator connected through a transmission line to an infinite bus.

In the jented case of a network with arbitrary PQ, PV and slack buses similar constraints are imposed on the injections, but these are much more complex to characterize. It is, however, essential for the loads, the generation levels, and the generator voltage levels to satisfy such constraints if the corresponding load flow is to exist in a sinusoidal steady state. Unfeasibility is therefore a 'condition equivalent to the non-existence of a real solution to the load flow equations. Such a condition can occur in practice or come "close" to occurring (with the consequent catastrophic effects, i.e. transient instability) if, for example, certain combinations of outages materialize. Thus, the loss of some major transmission lines coupled with the outage of some power plants. can lead to an unfeasible situation.

The general aims of this phase of the investigation have been to characterize and analyze those conditions under which the system's injections are feasible. Following the philosophy of security regions, we analyzed therefore the region of feasibility or steady state stability, $R_{z}$. This region is 
implicitly characterized by the set of injections, $\underline{z}$, for which a real voltage solution, $\underline{x}$, to the load flow equations exists. Thus

$$
\mathrm{R}_{\mathrm{z}}=\left\{\underline{\mathrm{z}} / \underline{\mathrm{z}}=\underline{\mathrm{L}}(\underline{\mathrm{x}}) \underline{\mathrm{x}}, \quad \underline{\mathrm{x}} \varepsilon \mathrm{R}^{\mathrm{n}}\right\}
$$

where $\underline{L}(\underline{x})$ x represents the right-hand side of the load flow equations (see Appendix A-6.Also [1] and [2]). Although in this study the feasibility region is a set in the space of injections, more generally the question of feasibility is dependent not only on injections, but on the network parameters as well.

An analysis of $R_{z}$ has been carried out exploiting the quadratic nature of the load flow equations in rectangular coordinates. This leads to the following general properties:

(1) $R_{z}$ is a cone stretching to infinity whose vertex is at the origin of the space of injections, $\underline{z}=\underline{0}$.

(2) $R_{z}$ lies on or above a set of supporting hyperplanes all passing through the origin.

(3) $R_{z}$ is convex.

These properties serve to characterize the nature of $\mathrm{R}_{z}$ in a general way, but they also lead to more explicit and'more, practical analytic relations describing steady state stability in terms of the injections. Property (1): $\mathrm{Rz}_{\mathrm{z}}$ is a cone

The proof of property (1) relies on the quadratic nature of the load flow equations in rectangular form (Appendix $A-6$ ), i.e. for each injection $z_{i}$,

$$
z_{i}=\underline{x}^{T} \underline{J}_{i} \underline{x}
$$


where $\underline{J}_{i}$ is a real symmetric matrix depending only on the network structure and on the type of buses in the load flow formulation (see Appendix $A-6$, background section). Thus, if $\underline{z}$ is a feasible vector of injections, $\rho \underline{z}$ where $\rho \geqslant 0$ is also feasible: This is so since $\underline{z}$ is feasible, there exists a real solution $\underline{x}$ to (1). Thus $\sqrt{\rho} \underline{x}$ from (1) is a solution to the load flow problem when the injections are $\rho \underline{z}$.

From this property it is clear that the membership of $\underline{z}$ in $R_{z}$ is independent of its "length". We could therefore intersect $R_{z}$ at some point and analyze only this intersection in vrder to study the properties of $R_{z}$. Such an intersecting surface could be the unit sphere, but as it turns out a plane is a more useful intersection device. This is only possibie if a plane can be found which intersects all the rays in $R_{z}$. The next property helps us to find such a plane.

Property' (2): Supporting Hyperplanes of $R_{Z}$

An assumption is made here peculiar to the standard formulation of the load flow, and that is the existence of at least one injection which corresponds to a bus voltage magnitude squared. Such an injection occurs, of course, at the slack bus. Furthermore, if the network has PV buses then each of these buses contributes one such type of injection. Say that $z_{1}$ is the injection corresponding to $v_{s l a c k}^{2}$, then for any $\underline{z} \varepsilon \mathrm{R}_{\mathrm{Z}}$

$$
(1,0,0 ; \ldots, 0) \underline{z} \geqslant 0
$$

which is of the form

$$
\underline{\alpha}^{T} \underline{z} \geqslant 0
$$


where $\underline{\alpha}$ is a constant vector of dimension equal to that of $\underline{z}$. Thus there exists at least one hyperplane of the form $\underline{\alpha}^{T} \underline{z}=$ o (i.e. passing through the origin) such that any vector $\underline{z} \varepsilon R_{z}$ lies "above" this plane in the sense of (3). It is not difficult to see that the hyperplane defined by (2) also touches, i.e. it is tangent to $R_{z}$. To see this choose an arbitrary set of voltages, $\underline{x}$, except for $e_{1}$ (note that $f_{1}=0$ ), which is set to zero. This defines a feasible vector with all the injections non-zero except for $z_{1}$ which becomes zero $\left(z_{1}=e_{1}{ }^{2}\right)$, converting (2) into an equality. Similar support hyperplanes can be defined from each PV bus. The support hyperplanes or feasibility planes thus obtained are not the most useful from the feasibility point of view. They simply state the obvious fact that the voltage magnitudes squared at the PV and slack buses must be positive. The support hyperplanes are nonetheless boundaries of $R_{z}$. What is more useful is to characturiae the boundaries of $R_{z}$ i.e. the feasibility constraints involving the real and reactive injections as well as the voltage magnitude injections. As was stated earlier, we are looking for a plane which will intersect all the rays in $R_{z}$ and hence all of its boundaries. Such a plane will take the form

$$
\underline{z}_{0}^{\mathrm{T}} \mathrm{z}=\mathrm{k}
$$

where $\underline{z}_{\circ}$ is a constant vector perpendicular to the plane, while $\mathrm{k}$ is some positive number.

Consider the simple example of a DC voltage source $\mathrm{V}$ feeding a real load $P$ through a resistor $R$. Then it is easy to show that a feasible load flow will exist if and only if 


$$
P \leqslant \frac{V^{2}}{4 R}
$$

and the obvious condition $\mathrm{v}^{2} \geqslant 0$. These inequalities define $\mathrm{R}_{\mathrm{z}}$ shown below,

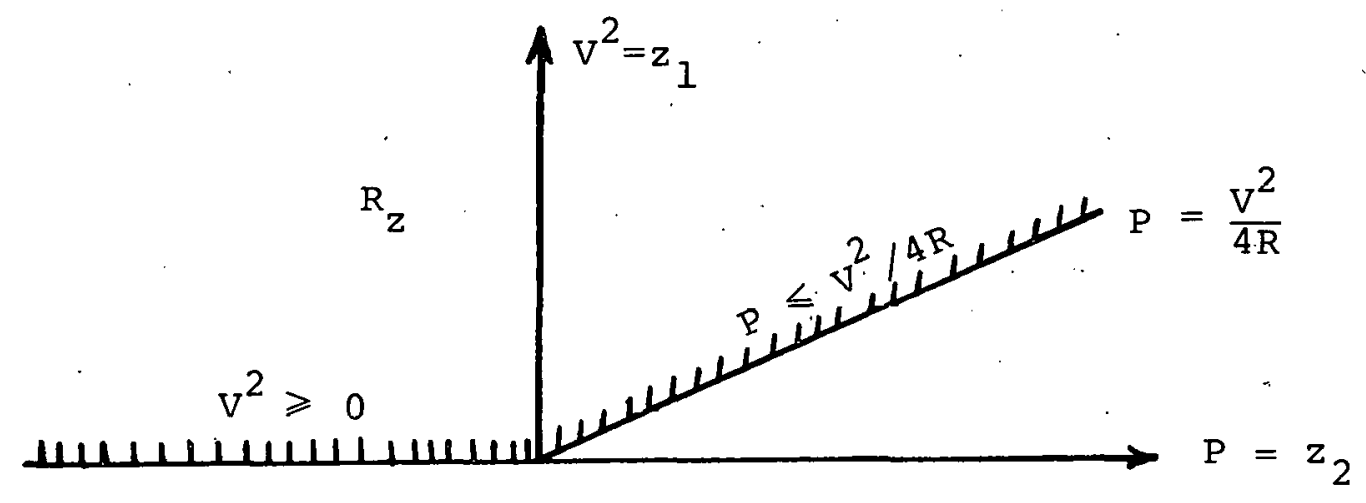

$\frac{R_{\text {if }} \text { for } a \cdot D C \text { source } V \text { feeding a real load } P}{\text { through a resistor } R}$

From this example we see that if $\underline{z}_{0}=(1,0)^{\mathrm{T}}$, the plane defined by (4) will intersect only one boundary of $R_{z}$, i.e. $\mathrm{P}=\mathrm{V}^{2} / 4 \mathrm{R}$. If $\underline{z}_{\mathrm{O}}=(0,-1)^{\mathrm{T}}$ only the boundary $\mathrm{V}^{2}=0$ will be intersected by the plane $\underline{z}_{0}^{T} \underline{z}=k$. If $\underline{z}_{0}=(1,-2 R)$, for example, the plane will intersect both boundaries. See below

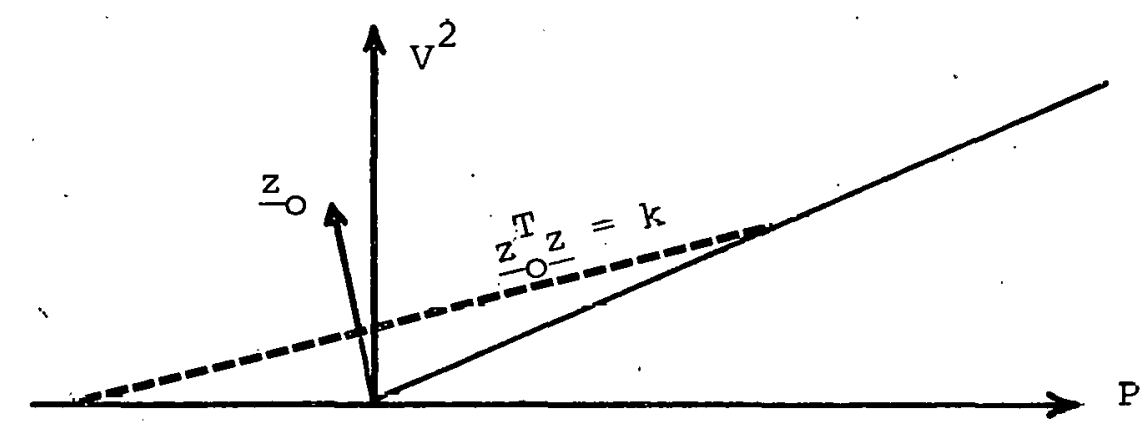

$$
\frac{\text { Plane } z}{\mathrm{Of} \mathrm{R}_{\mathrm{z}}}-\mathrm{T}=\mathrm{k} \text { intersecting both boundaries }
$$

Definition: The intersection of the plane $\underline{z}_{0}^{T} \underline{z}=k$ with $R_{z}$ defines a surface $P_{z O^{\circ}}$. This plane has $k>0$, and the property that every ray in $\mathrm{R}_{\mathrm{z}}$ intersects the plane.. 
In order to examine $\mathrm{R}_{\mathrm{z}}$ we can therefore analyze $\mathrm{P}_{\mathrm{zo}}$ instead.

One characteristic of such an intersecting plane is that since $k>0$ then for all $\underline{z} \varepsilon R_{z}$

$$
\underline{z}_{0}^{T} \underline{z}>0
$$

but from the properties of the load flow equation (Appendix A-6) this is equivalent to saying that for all real $\underline{x}$

$$
\underline{x}^{T} \cdot \underline{\underline{z}}\left(\underline{z}_{0}\right) \underline{x}>0
$$

which implies that $\underline{\mathrm{J}}\left(\underline{z}_{0}\right)$ is positive definite.

Thus we claim that finding a vector $\underline{z}_{0}$ such that $\underline{\mathrm{J}}\left(\underline{z}_{0}\right)>\underline{0}$ implies that the plane $\underline{z}^{\mathrm{T}} \underline{z}_{0}=\mathrm{k}>0$ intersects all rays in $R_{z}$. This is obviously true by considering that . for all real $\underline{x}$ (hence all $\left.\underline{z} \in R_{z}\right)$, since $\underline{J}\left(\underline{z}_{0}\right)>0$, then $\underline{x}^{T} \underline{J}\left(\underline{z}_{0}\right) \underline{x}>0$. In fact, we can always adjust the length of $\underline{x}$ (or of $\underline{z}$ ) so that $\underline{x}^{T} \underline{J}\left(\underline{z}_{0}\right) \underline{x}=k$ i.e. $\underline{z}_{0}^{T} \underline{z}=k$. This length is never infinite unless $\underline{z}$ is perpendicular to $\underline{z}_{0}$ ' but this is impossible for it would imply that for $\underline{z} \neq 0$ (i.e. $\underline{x} \neq \underline{o}), \underline{z}_{0}^{\mathrm{T}} \underline{z}=$ o or $\underline{x}^{\mathrm{T}} \underline{\mathrm{J}}\left(\underline{z}_{0}\right) \underline{\mathrm{x}}=0$ :

Now from the support hyperplanes defined by the voltage magnitude injections we can define at least a semidefinite matrix $\underline{\mathrm{J}}\left(\underline{z}_{0}\right)$. To see this let

$$
\underline{z}=\left[\begin{array}{l}
\underline{z}_{\mathrm{V}} \\
\underline{z}_{\mathrm{P}} \\
\underline{z}_{\mathrm{Q}}
\end{array}\right]
$$

where $\underline{z}_{V}$ represents the voltage injection, $\underline{z}_{P}$, the real powers injected, and $\underline{z}_{Q}$ ' the reactive powers injected: 
Similarly define

$$
\underline{z}=\left[\begin{array}{l}
\underline{z}_{V} \\
\underline{z}_{P} \\
\underline{z}_{Q}
\end{array}\right]
$$

where $\underline{z}_{\mathrm{V}}$ represents the voltage injection, $\underline{z}_{p}$, the real powers injected, and $\underline{z}_{Q}$, the reactive powers injected. Similarly define

$$
\underline{\alpha}=\left[\begin{array}{ll}
\underline{\alpha}_{\mathrm{V}} \\
\underline{\alpha} & \mathrm{P} \\
\underline{\alpha} & \mathrm{\theta}
\end{array}\right] .
$$

Thus if we let

$$
\underline{z}_{\circ}=\left[\begin{array}{l}
\underline{\alpha} \\
\underline{0} \\
\underline{0}
\end{array}\right]
$$

where the element of $\underline{\alpha}_{V}$ are all positive, $\underline{J}\left(\underline{z}_{0}\right)$ will be positive semi-definite.

'There exist a variety of ways of modifying the vector $\underline{z}_{0}$ defined by $(10)$ to make $\underline{\mathrm{J}}\left(\underline{z}_{0}\right)>$ o. An empirical choice that always seems to wurk well for typical power networks is to let all the elements of $\underline{\alpha}_{\mathrm{V}}$ equal one and all the elements of $\underline{\alpha}_{\mathrm{P}}$ and $\underline{\alpha}_{\mathrm{Q}}$ equal 0.1 .

A rigorous justification of this result, and a more systematic choice of $\underline{z}_{0}$ is being developed, and will be presented in the final report.

Figure 4.3 illustrates the intersection of $R_{z}$ with the plane $\underline{z}_{0}^{T} \underline{z}=k$ and the resulting feasibility surface $\mathrm{P}_{\mathrm{z}_{0}}$. 
Computation of arbitrary support hyperplanes

Arbitrary support hyperplanes can be found by

searching along $\mathrm{P}_{\mathrm{z}} \mathrm{o}$ in a direction defined by the arbitrary vector $\underline{z}_{1}$. This vector defines a plane $\underline{z}_{1}^{T} \underline{z}=c$ which intersects $P_{\text {zo }}$ for different values of $c$. As shown in Fig. 4.3 by varying $\mathrm{c}=\underline{\mathrm{z}}{ }_{\mathrm{I}}^{\mathrm{T}} \underline{\mathrm{z}}$ over $\mathrm{P}_{\mathrm{z}}$ o we obtain a maximum and minimum of $c$.

The search for the arbitrary feasibility plane $\underline{\alpha}^{\mathrm{T}} \underline{z}=0$ can then be considered as solving the following optimization problem.

$$
\begin{aligned}
& \operatorname{Max} \quad \underline{z}{ }_{1}^{T} \underline{z}=c \\
& s \in t \quad \underline{z}_{0}^{T} \quad \underline{z}=k
\end{aligned}
$$

The injection $\underline{z}$ can be put into a quadratic form $[1,2]$

$$
z_{i}=\underline{x}^{T} \underline{J}_{i} \underline{x}=1, \ldots, 2 \mathrm{~N}
$$

where $z_{i}$ is the $i$ th. element of the injection $z$ and $\underline{x}^{T}=$ $\left[\underline{c}^{\mathrm{T}}, \underline{f}^{\mathrm{T}}\right]$ is the vertor of bus voltaqes in rectangular coordinates. $\underline{J}_{i}$ is a function of the network admittance $\underline{Y}$ and the type of bus, while $\mathrm{N}$ is the number of buses.

$$
\text { Using equation (12) we form }
$$

$$
\underline{z}_{1}^{T} \underline{z}=\underline{x}^{T}\left[\underline{J}\left(\underline{z}_{I}\right)\right] \underline{x}
$$

where

$$
\underline{J}\left(\underline{z}_{1}\right)=\sum_{i=1}^{N} \underline{J}_{i} z_{l i} \quad \text {, a real symmetric matrix. }
$$

Substituting (13) into (11) gives the following form

$$
\begin{aligned}
& \underset{\operatorname{Max}}{\operatorname{x}} \underline{x}^{T} \underline{J}\left(\underline{z}_{1}\right) \underline{x}=c \\
& \text { s.t } x^{T} \underline{J}\left(\underline{z}_{0}\right) \underline{x}=k
\end{aligned}
$$

Forming the Lagrangian $[1,4]$

$$
L=\underline{x}^{T} \underline{J}\left(\underline{z}_{1}\right) \underline{x}+\lambda\left(k-\underline{x}^{T} \underline{J}\left(\underline{z}_{0}\right) \underline{x}\right)
$$


and differentiating it with respect to $\mathrm{x}$ gives the generalized eigenvalue problem

$$
\left[\underline{J}\left(\underline{z}_{1}\right)-\lambda \underline{J}\left(\underline{z}_{0}\right)\right] \underline{x}=\underline{o}
$$

- At an extreme value as defined by (15),

$$
\begin{aligned}
c & =\underline{x}^{T} \underline{J}\left(\underline{z}_{1}\right) \underline{x} \\
& =\lambda \underline{x}^{T} \underline{J}\left(z_{0}\right) \underline{x} \\
& =\lambda k
\end{aligned}
$$

Thus the maximum and minimum of $c$ are given by the maximum and minimum eigenvalues of (15). The maximum eigenvalue, $\lambda_{\max }$ defines the following condition for all real $\underline{x}$

$$
c=\underline{x}^{T} \underline{J}\left(\underline{z}_{l}\right) \underline{x} \leqslant \lambda_{\max } \underline{x}^{T} \underline{J}\left(\underline{z}_{0}\right) \underline{x}
$$

or for $\operatorname{all} \underline{z} \varepsilon \mathrm{R}_{z^{\prime}}$

$$
\left(\lambda \max \underline{z}_{0}-\underline{z}_{1}\right)^{\mathrm{T}} \underline{z}: \geqslant 0
$$

Similarly for all $\underline{z} \varepsilon \mathrm{R}_{\mathrm{z}}$

$$
\left(\underline{z}_{1}-\lambda_{\min } \underline{z}_{0}\right)^{T_{z}} \geqslant 0
$$

Equations (17) and (18) represent the two feasibility constraints associated with the arbitrary direction vector $\underline{z}_{1}$. The fact that $\underline{J}\left(\underline{z}_{0}\right)$ is positive definite guarantees the existence of global maxima and minima to (14). The eigenvectors corresponding to $\lambda_{\max }$ and $\lambda_{\min } \underline{x}_{\max }$ and $\underline{x}_{\min }$, define 
through the load flow equation the vectors $\underline{z}_{\mathrm{bmax}}$ and $\underline{\underline{z}}_{\underline{b} \min }$ tangent to $R_{z}$ and belonging to the support hyperplanes defined by (17) and (18).'

As long as $\underline{J}\left(\underline{z}_{0}\right)$ is positive definite all support hyperplanes can be found by the above mentioned technique. We define the set $R_{\alpha}$ as the set of all the vectors $\underline{\alpha}$ defining $a$, support hyperplane, $\underline{\alpha}^{\mathrm{T}} \underline{z}=0$ with $\underline{\alpha}^{\mathrm{T}} \underline{z} \geqslant 0$ for all $\underline{z} \varepsilon \mathrm{R}_{z}$. Thus

$$
R_{X} \triangleq\left\{\underline{\alpha} / \underline{\alpha}^{T} \underline{z} \geqslant 0, \underline{z} \varepsilon R_{z}^{\cdot}\right\}
$$

Property 3: Convexity of $\mathrm{R}_{\mathrm{Z}}$

The convexity of $R_{z}$ is important if we are to use the feasibility planes to characterize $R_{z}$. Consider the region $\mathrm{R}_{\mathrm{z}}$.'Suppose. that its intersection has the shape shown below. We show two support hyperplanes, one of which touches $R_{z}$ at one point where the region is locally convex, and one where it touches at two points where the region is locally concave, $\underline{z}_{\mathrm{b} 1}$ and $\underline{z}_{\mathrm{b} 2}$. Let the corresponding eigenvectors be $\underline{x}_{1}$ and $\underline{x}_{2}$. Now define the voltage vector $\underline{x}(s)$,

$$
\underline{\underline{x}}(s)=s \underline{x}_{1}+(I-s) \underline{x}_{2}
$$

The corresponding injection is then 


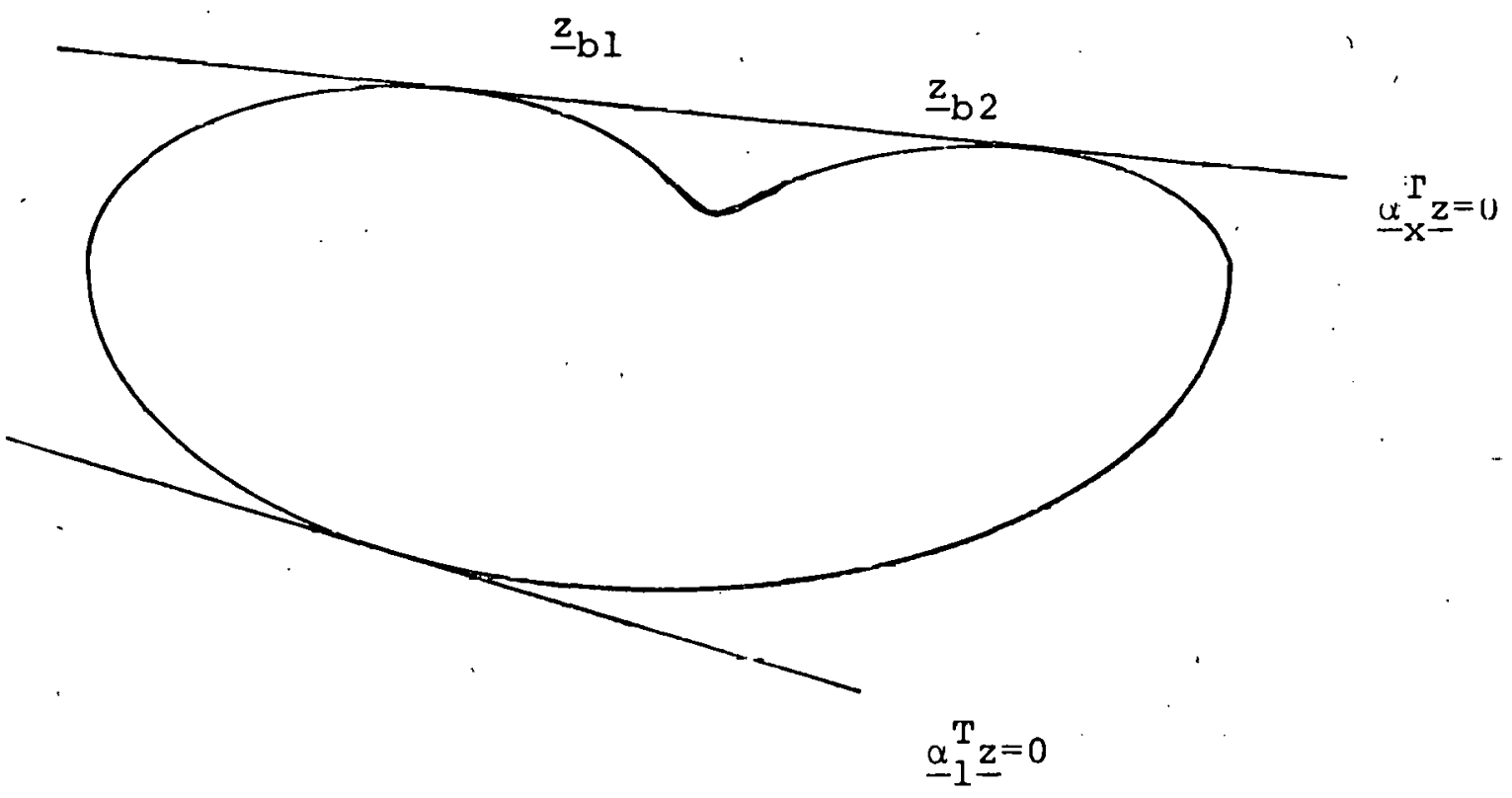

Figure used for the convexity argument. 


$$
\begin{aligned}
\underline{z}(s) & =s^{2} \underline{L}\left(\underline{x}_{1}\right) \underline{x}_{1}+2 s(1-s) \underline{L}\left(\underline{x}_{1}\right) \underline{x}_{2}+(1-s)^{2} \underline{L}_{\left(x_{2}\right) \underline{x}_{2}} \\
& =s^{2} \underline{z}_{b 1}+2 s(1-s) \underline{L}\left(\underline{x}_{1}\right) \underline{x}_{2}+(1-s)^{2} \underline{z}_{b} 2
\end{aligned}
$$

Now since $\underline{z}_{b 1}$ and $\underline{z}_{b 2}$ belong to $\underline{\alpha}^{T} \underline{z}=0$, consider

$$
\underline{\alpha}^{T} \underline{z}(s)=2 s(1-s) \underline{\alpha}^{T} \underline{L}\left(\underline{x}_{1}\right) \underline{x}_{2}
$$

But

$$
\underline{\alpha}^{T} \underline{\underline{x}}\left(\underline{x}_{1}\right)=\underline{J}(\underline{\alpha}) \underline{x}_{1}
$$

and since $\underline{x}_{1}$ is an eigenvector of (15) with $\underline{\alpha}=\underline{z}_{1}-\lambda_{\min }$ or $\lambda_{\max } \underline{z}_{0}-\underline{z}_{1}$, then

$$
\underline{J}(\underline{\alpha}) \underline{x}_{1}=\underline{0}
$$

Thus for all s

$$
\underline{\alpha}^{\mathrm{T}} \underline{z}(\mathrm{~s})=0
$$

It follows then that $\underline{z}(s)$ is a feasible vector lying on the plane $\underline{\alpha}^{\mathrm{T}} \underline{\mathrm{z}}=\mathrm{o}$ for all s. By varying $\mathrm{s}$ from 0 to 1 we see that $\underline{z}(s)$ moves continuously from $\underline{z}_{b 1}$ to $\underline{z}_{b 2}$ inside $R_{z}$. The assumed non-convexity is therefore impossible.

By this argument and the empirical data obtained so far we therefore make the hypothesis that $R_{z}$ is convex. 


\subsection{Closest Boundary Injection}

Description and Theory

The complete characterization of the feasibility region $\mathrm{R}_{\mathrm{z}}$ would provide the necessary and sufficient conditions for steady state stability or feasibility. This, however, requires the identification of the infinitely many supporting hyperplanes circumscribing the convex cone $\mathrm{R}_{z}$, which is an impossible task. However it is possible to characterize that part of the feasibility region $\mathrm{R}_{\mathrm{z}}$ which is in the neighbourhoud. $u f$ an operating injection $\underline{z}_{g}$.

Essentially what is required is the particular boundary injection $\underline{z}_{b}^{*}$ which is closest to the given operating injection $\underline{z}_{g}$. The closest boundary injection $\underline{z}_{b}^{*}$ lies on the corresponding closest supporting hyperplane $\underline{\alpha}_{* \underline{z}}^{T}=0$ as illustrated for a 2- and 3-dimensional case in Fig. 4.1. The closest boundary injection can be defined such that the angle between $\underline{z}_{b}^{*}$ and $\underline{z}_{g}$ is minimum, which implies

$$
\cos \gamma^{*}=\left\{\frac{\underline{z}_{g}^{T} \underline{z}_{b}^{*}}{\left\|\underline{z}_{g}\right\|\left\|\underline{z}_{b}^{*}\right\|}\right\}=\underset{\underline{z}_{b}}{\max } \cos \gamma=\underset{\underline{z}_{b}}{\max }\left\{\frac{\underline{z}_{g}^{T} \underline{z}_{b}}{\left\|\underline{z}_{g}\right\|\left\|\underline{z}_{b}\right\|}\right\}
$$

where $\underline{z}_{b}{ }^{\prime} \varepsilon \operatorname{Ext}\left(R_{z}\right)$, the set of injections lying on the boundary of $R_{z}$.

The cosine of the angle between $\underline{z}_{g}$ and $\underline{z}_{b}$ cannot be maximized over arbitráry $\underline{z}$ since this maximum would occur at 


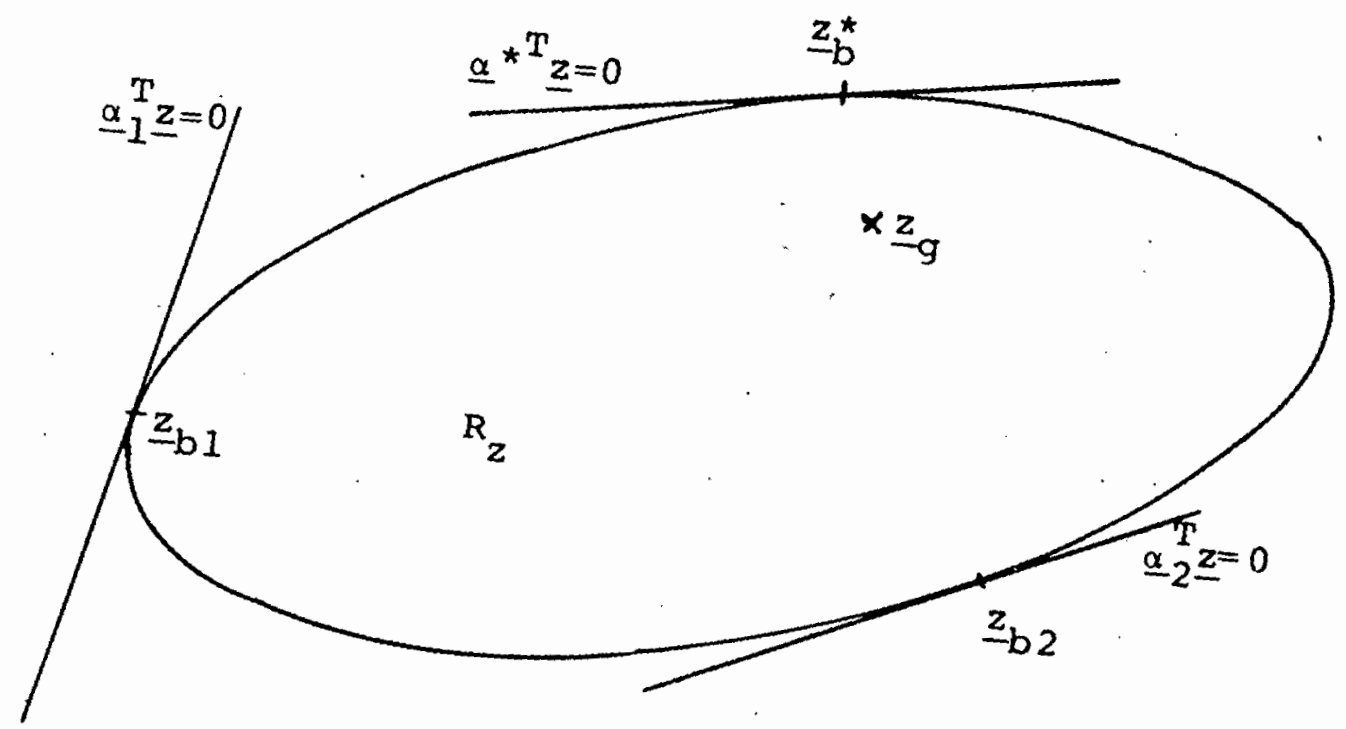

2-D Feasibility Region

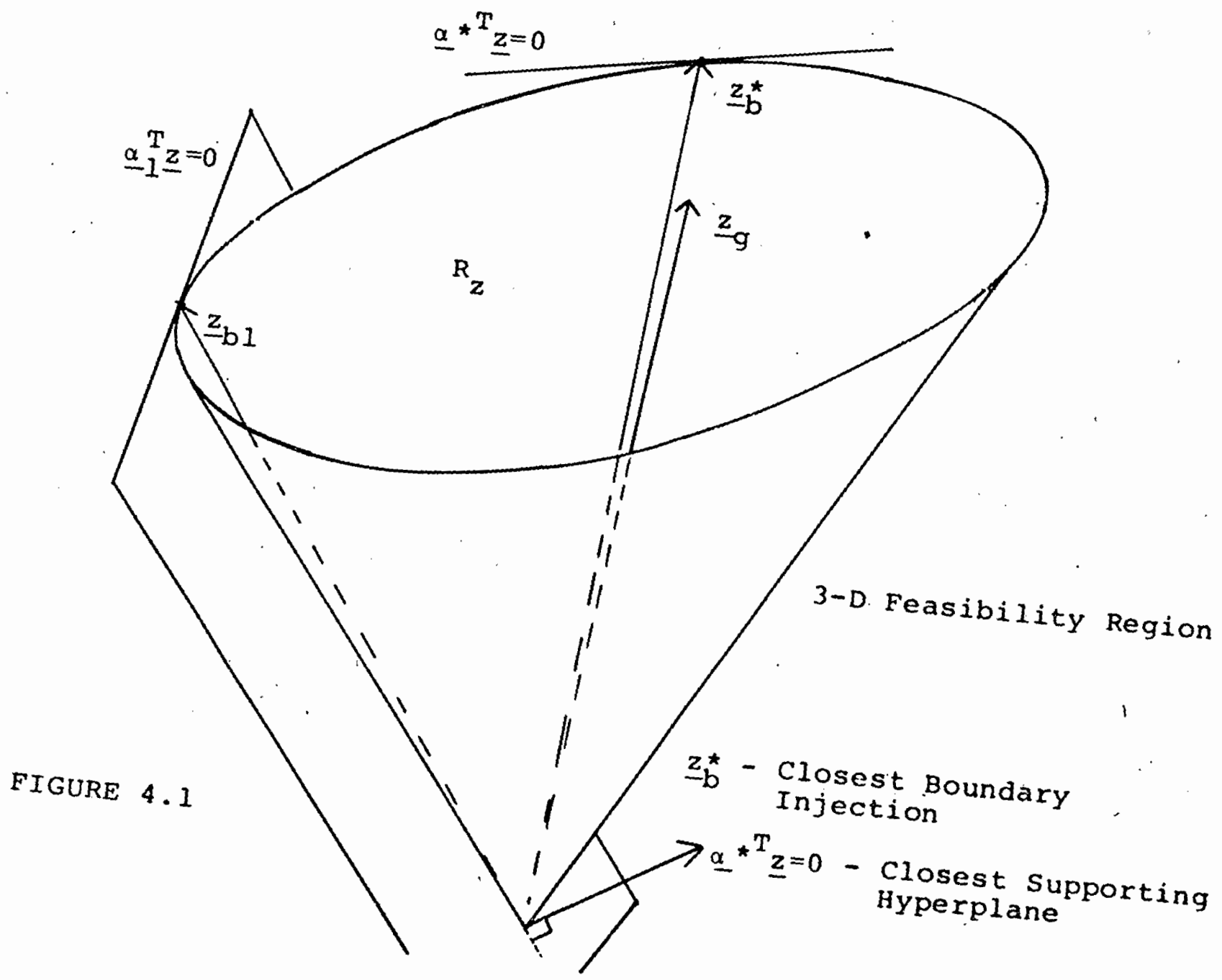


$\gamma=0$, i.e. the optimum $\underline{z}$ would equal $\underline{z}_{g}$ if $\underline{z}_{g} \cdot{ }^{\varepsilon}{ }_{z} \cdot$ If $\underline{z}_{g}$ $\$ R_{z}$, this technique will work, but since it is required to find the closest boundary injection to a typical operating point, i.e. $\underline{z}_{g} \varepsilon R_{z}$, a technique which is applicable to $\underline{z}_{g}$ both inside and outside $\mathrm{R}_{z}$ is more appropriate.

A more useful feasibility measure is the cosine of the angle between the normal of the supporting hyperplane $\underline{\alpha}$ and $\underline{z}_{g}$, which is of the form

$$
\cos \theta=\frac{\alpha^{\mathrm{T}}}{\|\underline{\alpha}\| \underline{z}_{\underline{y}}}\left\|\underline{\underline{z}}_{g}\right\|
$$

This'is depicted geometrically in Figs. 4.1 and 4.2 . To find the $\underline{z}_{b}^{*}$ which is closest to $\underline{z}_{g^{\prime}}$ a vector $\underline{\alpha}^{*}$.must be chosen from the set $R_{\alpha}$ such that its angle to $\underline{z}_{g}$ is largest. Once $\underline{a}^{*}$ is located, $\underline{z}_{b}^{*}$ is available from which the measure of closeness of $\underline{z}_{\mathrm{g}}$ to $\underline{\mathrm{z}}_{\mathrm{b}}$ can be easily computed. Indeed this information is immediately available from Equation (27).

As illustrated in Fig. 4.2, if $\underline{z}_{g} \varepsilon R_{z}$, the angle $\theta$ * between $\underline{z}_{g}$ and $\underline{\alpha}^{*}$ is less than $90^{\circ}$, which implies that $\cos \theta$ * is positive. If $\underline{z}_{g} \notin R_{z}$, the angle $\theta *$ is greater than $90^{\circ}$ and thus $\cos \theta *$ is negative. (This is necessarily true since the conical region $R_{z}$ lies at least above a plane and is convex,) So.now a measure exists which distinguishes between feasible and unfeasible $\underline{z}$. Thus if $\underline{z}_{g l}$ is closer to the boundary than $\underline{z}_{\mathrm{g} 2}$ ' then $\cos \theta_{1}^{*}$ will be less than $\cos \theta_{2}^{*} \cdot$ 

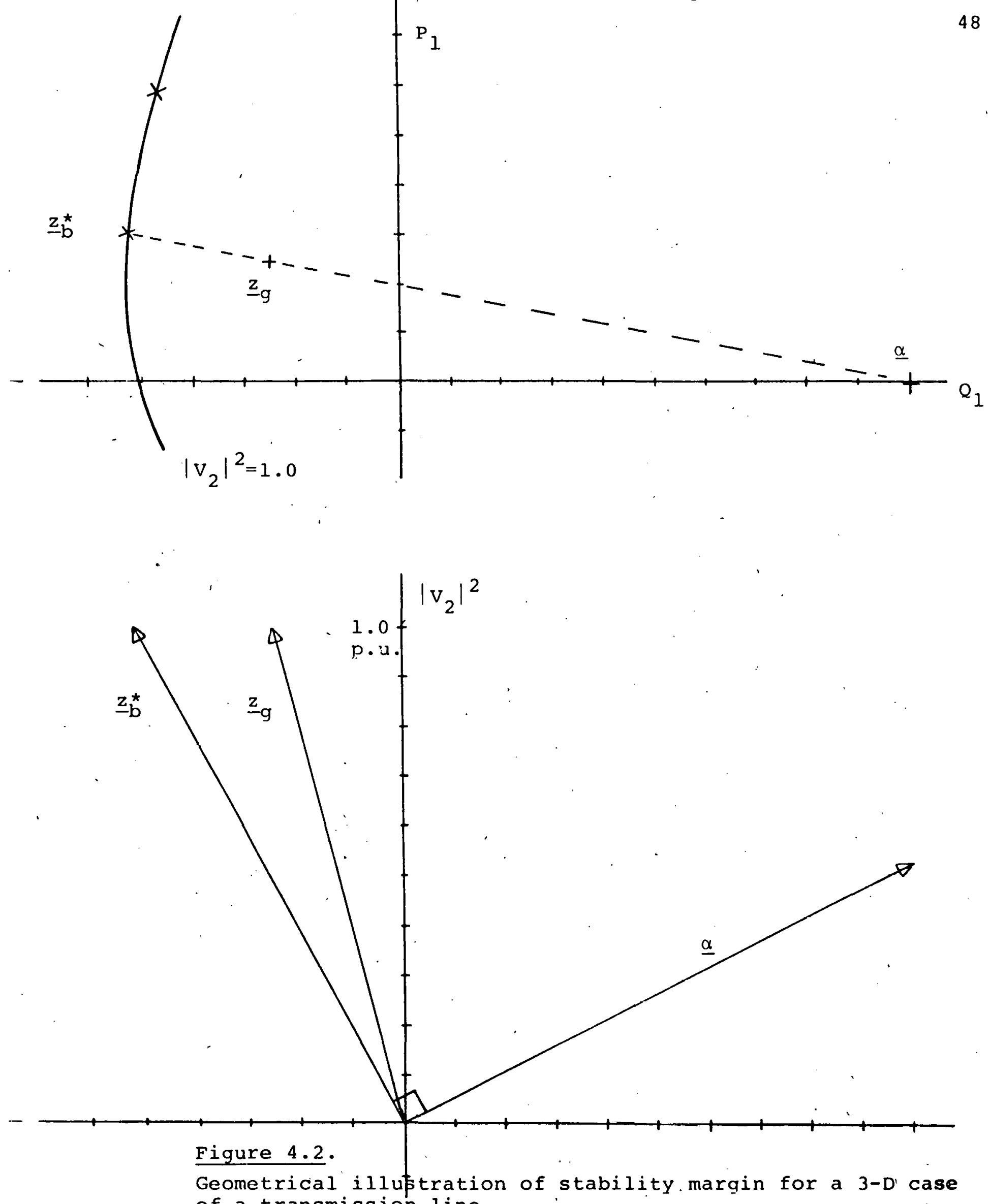
of a transmission line. 
To characterize the feasibility 'region about $\underline{z}_{g}$ and to locate the closest boundary injection, the following optimization problem must then be solved.

$$
\begin{aligned}
\text { S.M. } & =\cos \theta *=\frac{\underline{\alpha}^{* T} \underline{z}_{g}}{\left\|\underline{\alpha}^{\star}\right\|}\left\|\underline{z}_{g}\right\| \\
= & \min _{\underline{\alpha}_{\varepsilon R}} \frac{\underline{\alpha}^{\mathrm{T}}}{\|\underline{\alpha}\|} \underline{\underline{z}}_{g}\left\|\underline{z}_{g}\right\|
\end{aligned}
$$

where S.M. is defined as the steady state stability margin or the feasibility margin of $\underline{z}_{g}$.

Computation of Stability Margin - Theory

Now that a technique is available to find the supporting hyperplanes, we can proceed to compute the stability margin, S.M. The objective function of the corresponding optimization scheme is of the form

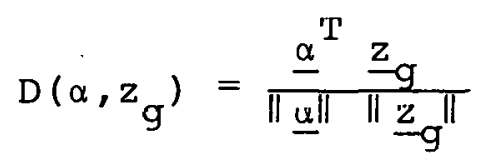

where

$$
\begin{aligned}
\text { S.M. }= & \min _{\underline{\alpha} \varepsilon R_{\alpha}} D\left(\underline{\alpha}_{\alpha}, \underline{z}_{g}\right) \\
= & \min _{\underline{\alpha} \varepsilon R_{\alpha}} \underline{\alpha}^{\mathrm{T}} \underline{\underline{z}}\left\|\underline{z}_{g}\right\|
\end{aligned}
$$


Numerical optimization techniques $[4,5,6]$ are available which compute the stability margin, S.M., without requiring an analytical expression for the gradient function. However the availability of an explicit gradient function allows more powerful numerical scheme with excellent convergence properties $[4,5,6]$ to be used.

The derivative of the objective function in Equation (29) can be shown to be of the form

$$
\frac{d D}{d \underline{\alpha}}=\frac{i}{\|\alpha\|}\left[\underline{I}-\frac{\underline{\alpha} \underline{\alpha}^{T}}{\underline{\alpha}^{T} \underline{\alpha}}\right] \frac{\underline{z}_{g}}{\left\|\underline{z}_{g}\right\|}
$$

The constant vector $\underline{\alpha}$ defining the supporting hyperplane is of the form.

$$
\begin{aligned}
& \underline{\alpha}=\lambda \underline{z}_{0}-\underline{z}_{1} \text { if } \lambda=\lambda_{\max } \\
& =\underline{z}_{1}=\lambda_{2} \text { if } \lambda=\lambda_{\min }
\end{aligned}
$$

In searching for the stability margin using the objective function in (31) and the gradient in (32), the vector $\underline{\alpha}$ must always belong to $R_{\alpha}$, the set of supporting hyperplanes. In its present form, the optimization scheme using (32) as the gradient requires a constraint function restricting the movement of $\underline{\alpha}$ to the set $R_{\alpha}$. An alternative scheme which circumvents the need of a constraining function on $\underline{\alpha}$ is to minimize the objective function over $\underline{z}_{1}$, the vector defining the plane which cuts the feasibility surface $P_{z 0}$ as shown in 


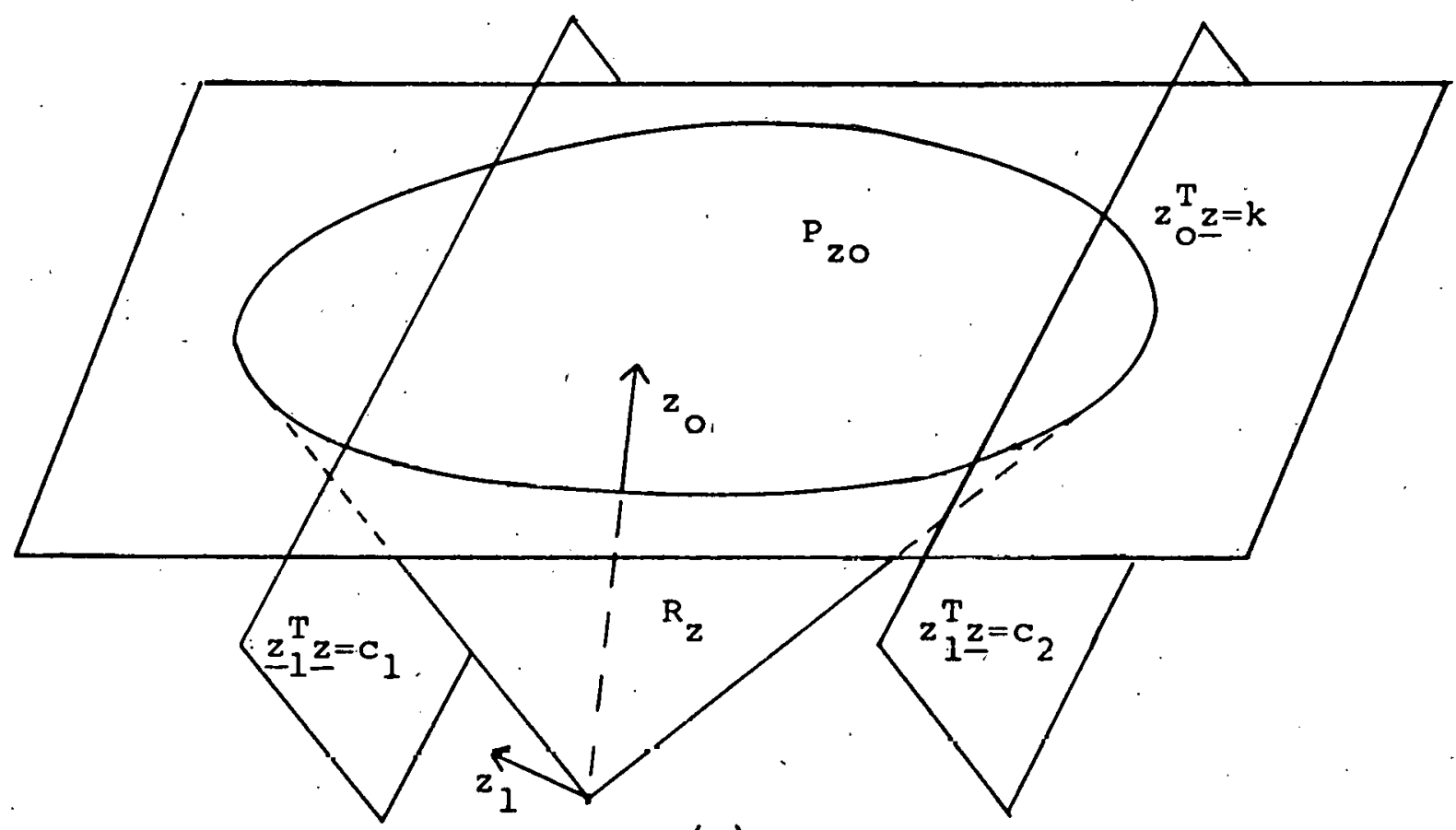

(a)

Illustration of the feasibility surface $P_{z O^{\circ}}$.

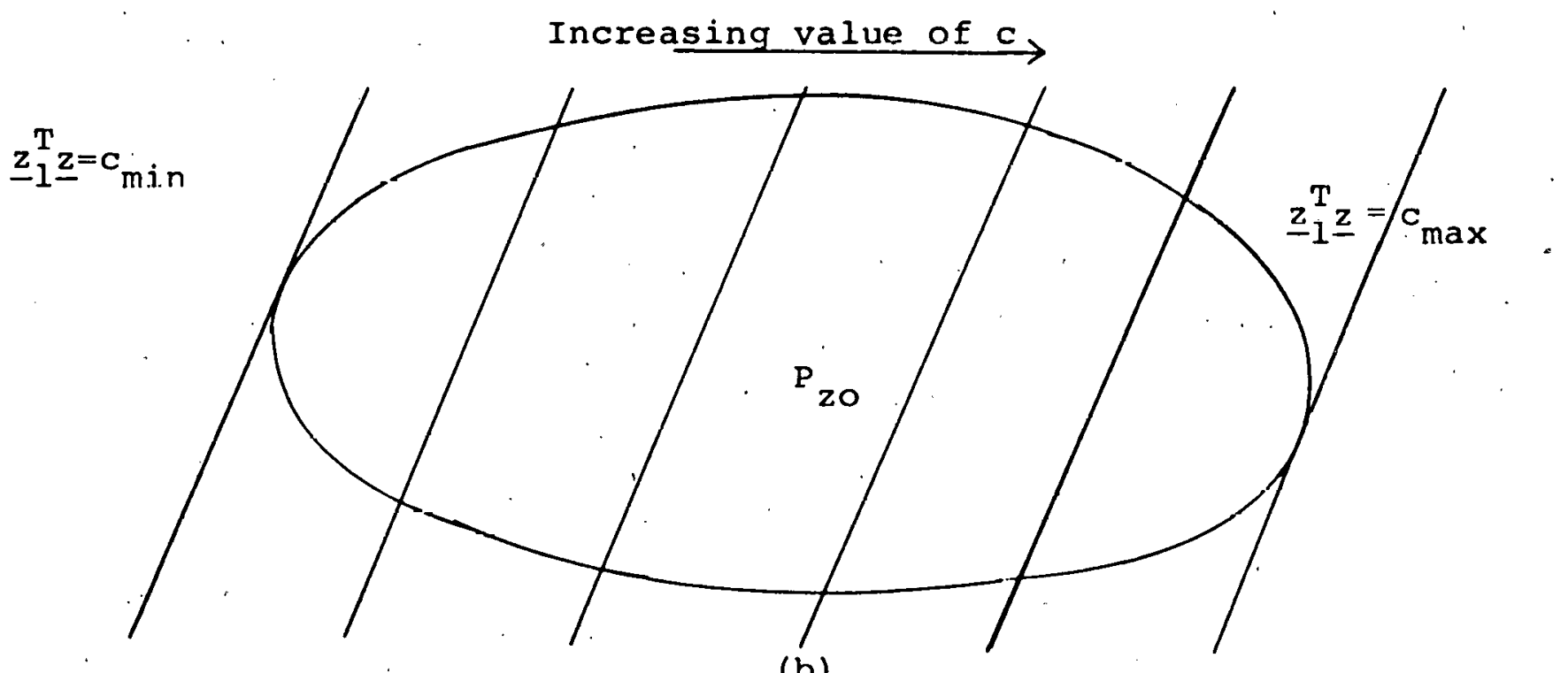

(b)

FIGURE 4.3. Inlersection of the plane $\underline{z}_{1}^{T} \underline{z}=c$ with ${ }_{z o}$ for
various values of $c$. 


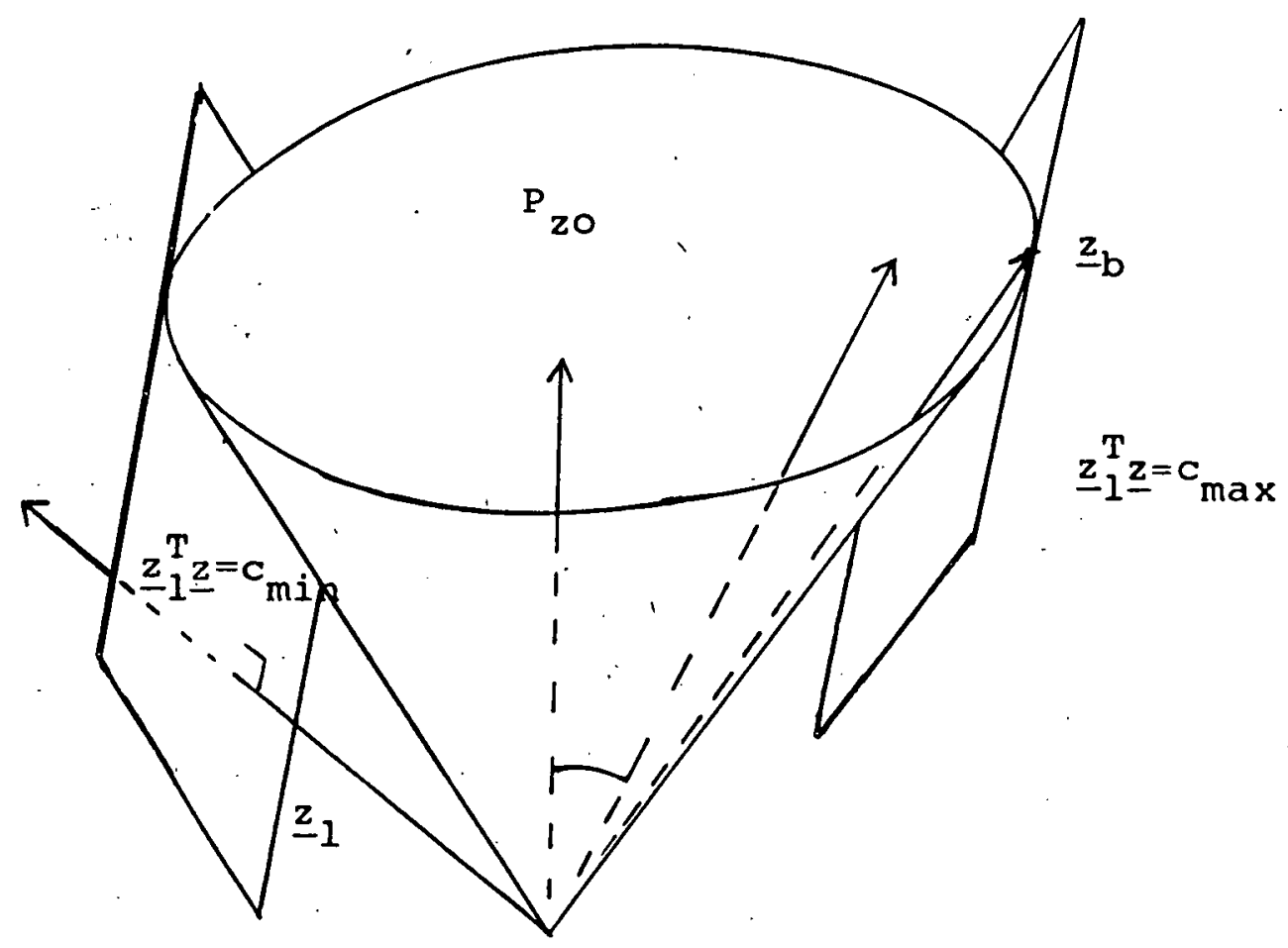

(a)

3-D Feasibility Region.

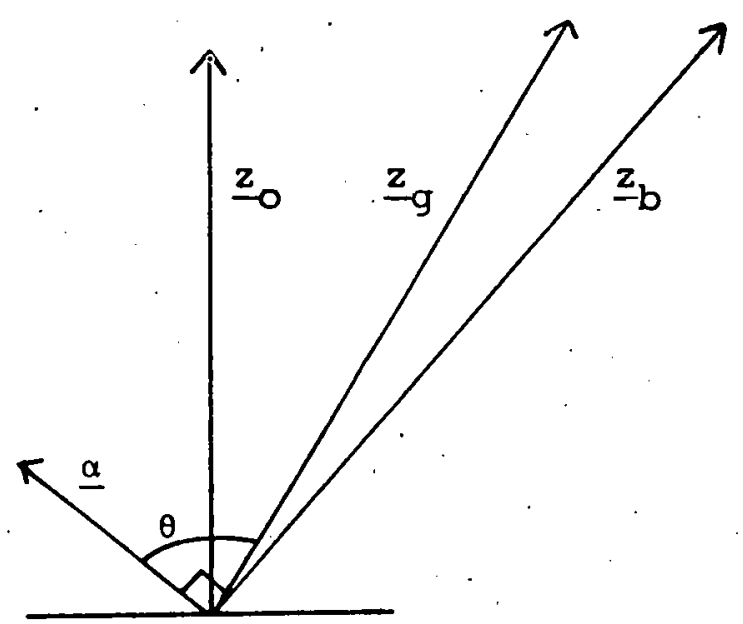

(b)

'A section of the feasibility region.

FIGURE 4.4 . 
Fig. 4.3.

Differentiating $\underline{\alpha}$ in (33) with respect to $\underline{z}_{1}$ for $\lambda=$ $\lambda_{\max }$ gives

$$
\mathrm{d}_{\underline{\alpha}}=\mathrm{d} \lambda \underline{\mathrm{z}}_{0}-\mathrm{d}_{\underline{z}}
$$

Upon premultiplication by $\underline{z}_{b^{\prime}}^{T}$ (34) takes the form

$$
\underline{\mathrm{z}}_{\mathrm{b}}^{\mathrm{T}} \mathrm{d} \underline{\alpha}=\mathrm{d} \lambda \underline{\mathrm{z}}_{\mathrm{b}}^{\mathrm{T}} \underline{\mathrm{z}}_{0}-\underline{\mathrm{z}}_{\mathrm{b}}^{\mathrm{T}} \underline{\mathrm{z}}_{1}
$$

We now need the following identitises

$$
\mathrm{J}(\underline{\alpha}) \underline{\mathrm{x}}_{\mathrm{b}}=0
$$

and

$$
\underline{\mathrm{z}}_{\mathrm{b}}^{\mathrm{T}} \mathrm{d} \underline{\alpha}=0
$$

From the generalized eigenvalue problem of (15), at $\lambda=\lambda_{\max }$ or $\lambda=\lambda_{\text {min' }}$, we must necessarily have

$$
\left[\underline{J}\left(\underline{x}_{1}\right)-\lambda \underline{J}\left(\underline{z}_{0}\right)\right] \underline{x}_{b}=0
$$

where $\underline{x}_{b}$ is the corresponding eigenvector. Thus we can form from (38)

$$
\underline{J}(\underline{\alpha}) \underline{x}_{b}=0
$$

where $\underline{\alpha}=\underline{z}_{1}-\lambda \underline{z}_{0}$ for $\lambda=\lambda_{\max }$. Before we proceed to use (3.6), to deduce the identity 
of (37), we pause to consider the significance of the identity of (36). The bus injections of a power system network can be put into the standard cartesian coordinate loadflow form $[1,2$, 7]

$$
\underline{z}=\underline{L}(\underline{x}) \underline{x}
$$

Premultiplying (40) by $\underline{\alpha}^{\mathrm{T}}$ and setting $\underline{z}=\underline{z}_{\mathrm{b}}$ and $\underline{\mathrm{x}}=\mathrm{x}_{\mathrm{b}}$ corresponding to the injection on the boundary of $R_{z}$ we have

$$
\begin{aligned}
\underline{\alpha}^{\mathrm{T}} \underline{\mathrm{z}}_{\mathrm{b}} & =\underline{\alpha}^{\mathrm{T}} \underline{\mathrm{L}}\left(\underline{x}_{\mathrm{b}}\right) \underline{\mathrm{x}}_{\mathrm{b}} \\
& =\underline{x}_{\mathrm{b}}^{\mathrm{T}} \underline{\mathrm{J}}(\underline{\alpha}) \underline{x}_{\mathrm{b}} \\
& =0
\end{aligned}
$$

by virtue of the definition of $\underline{z}$ in (12) and (13), and the identity of (36). We thus conclude from (41), that the stabillty margin uf any injection whish 1 ifs on Ext( $\left.R_{z}\right)$, the boundary of $R_{z}$ is zero. Since $\underline{z}$ must belong to $R_{z}$ for it to be feasible which implies $\underline{\alpha}^{T} \underline{z} \geqslant 0$, thus under the condition that the feasibility region $R_{z}$ is convex, the stability mar-

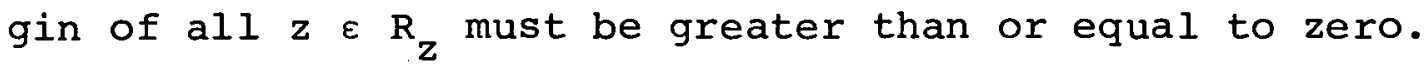
Similarly if $\underline{z} \notin R_{z}$, its stability margin is negative which agrees well with our earlier geometrical interpretations. The derivation of the identity $\underline{z}_{b}^{\mathrm{T}} \mathrm{d} \underline{\alpha}=0$ in (37) is done by considering small perturbations in the identity of (36), which implies 


$$
\underline{J}(\underline{\alpha}) \underline{x}_{b}+\underline{J}(\underline{\alpha}) d \underline{x}_{b}=0
$$

Premultiplying (42) by $\mathrm{x}_{\mathrm{b}}^{\mathrm{T}}$ gives

$$
\underline{x}_{b}^{T} J \cdot(a \underline{\alpha}) \underline{x}_{b}+\underline{x}_{b}^{T} \underline{J}(\underline{\alpha}) d \underline{x}_{b}=0
$$

But by (36) $\underline{x}_{b}^{T} \underline{J}(\alpha)=0$, which yields

$$
\underline{x}_{b}^{T} \underline{J}(d \underline{\alpha}) \underline{x}_{b}=0
$$

which givies the desired result

$$
\underline{\mathrm{z}}_{\mathrm{b}}^{\mathrm{T}} \mathrm{d} \underline{\underline{\alpha}}=\mathrm{o}
$$

Consider again the differential forms of $\underline{\alpha}$ in (35)

$$
\underline{z}_{\mathrm{b}}^{\mathrm{T}} \mathrm{d} \underline{\alpha}=\mathrm{d} \lambda \underline{\mathrm{z}}_{\mathrm{b}}^{\mathrm{T}} \underline{\mathrm{z}}_{\mathrm{O}}-\underline{\mathrm{z}}_{\mathrm{b}}^{\mathrm{T}} \mathrm{dz} \underline{z}_{1}
$$

which upon substituting for the identity in (43) and rearranging gives

$$
\mathrm{d} \lambda=\frac{\underline{\mathrm{z}}_{\mathrm{b}}^{\mathrm{T}} \mathrm{dz} \underline{\mathrm{z}}_{1}}{\underline{\mathrm{z}}_{\mathrm{b}}^{\mathrm{T}} \underline{\mathrm{z}}_{\mathrm{O}}}
$$

We can now find $\mathrm{d}_{\underline{\alpha}}$ by substituting (45) into (34) which is of the form

$$
\left.\mathrm{d} \underline{\alpha}=\frac{\underline{z}_{0} \underline{z}_{b}^{T}}{\underline{z}_{0}^{T} \underline{z}_{b}}-I\right] d \underline{z}_{1}
$$


Replacing $\mathrm{d} \underline{\alpha}$ in (32) of the original gradient function by gives the required gradient function with respect to $\underline{z}_{1}$.

$$
\frac{\partial \mathrm{D}}{\partial \underline{z}_{1}}=\frac{1}{\|\underline{\alpha}\|}\left[\frac{\underline{z}_{b} \underline{z}_{0}^{\mathrm{T}}}{\underline{z}_{0}^{\mathrm{T}} \underline{z}_{\mathrm{b}}}-I\right]\left[I-\frac{\underline{\alpha} \underline{\alpha}^{\mathrm{T}}}{\underline{\alpha}^{\mathrm{T}} \underline{\alpha}}\right] \frac{\underline{z}_{\mathrm{g}}}{\| \underline{z} \underline{g}^{\prime}}
$$

and can be written in the form

$$
\frac{\partial \mathrm{D}}{\partial \underline{z}_{1}}=\frac{1}{\|\underline{\alpha}\| \underline{z}_{g} \|}\left[\mathrm{T}_{1-\underline{z}_{\mathrm{b}}}+\mathrm{T}_{2} \underline{\alpha}-\mathrm{z}_{\mathrm{g}}\right]
$$

where scalars $\mathrm{T}_{1}$ and $\mathrm{T}_{2}$ are respectively

$$
\mathrm{T}_{I}=\frac{\left(\underline{z}_{0}^{\mathrm{T}} \underline{z}_{g}\right)}{\left(\underline{z}_{0}^{\mathrm{T}} \underline{z}_{b}\right)}-\frac{\left(\underline{z}_{0}^{\mathrm{T}} \underline{\alpha}\right)}{\left(\underline{z}_{0}^{\mathrm{T}} \underline{z}_{b}\right)} \frac{\left(\underline{\alpha}^{\mathrm{T}} \underline{z}_{g}\right)}{\left(\underline{\alpha}^{\mathrm{T}} \underline{\alpha}\right)}
$$

and

$$
\mathrm{T}_{2}=\frac{\underline{\alpha}^{\mathrm{T}} \underline{\mathrm{z}} \mathrm{g}}{\underline{\alpha}^{\mathrm{T}} \underline{\alpha}}
$$

At the minimum the gradient vanishes, $\partial \mathrm{D} / \partial \underline{z}_{1}=\varrho$ which implies

$$
\underline{\mathrm{z}}_{\mathrm{g}}=\mathrm{T}_{1} \underline{\mathrm{z}}_{\mathrm{b}}+\mathrm{T}_{2} \underline{\alpha}
$$

We thus conclude that at the minimum, the vectors $\underline{z}_{g} \underline{z}_{b}$ and $\underline{\alpha}$ Iie in the same plane as illustrated geometrically earlier for a 3-dimensional case in Figs. 4.1 and 4.2 .

In a particular direction of search $\underline{z}_{1}$ to solve for the stability margin, S.M., using the objective function in (30), 
there are.two extremum solutions to the generalized eigenvalue problem in (15) and thus giving rise to two possibie $\underline{\alpha}^{\prime} \mathrm{s}$ as indicated in (33). In the derivation of the gradient function, we have only considered $\underline{\alpha}$ corresponding to the maximum eigenvalue of the form

$$
\underline{\alpha}=\lambda \underline{z}_{0}-\underline{z}_{1}
$$

The other candidate of $\underline{\alpha}$ which must be considered is the one corresponding to the minimum eigenvalue, i.e. $\lambda=\lambda_{\min }$ which has the torm

$$
\underline{\alpha}=\underline{z}_{i}-\lambda \underline{z}_{0}
$$

Using a similar. analysis, it is not difficult to show that the gradient of the objective function of (30) has the following expression

$$
\frac{\partial \mathrm{D}}{\partial \underline{z}_{1}}=\frac{1}{\|\underline{\alpha}\|\left\|\underline{z}_{g}\right\|}\left[\underline{z}_{g}-\underline{z}_{b} T_{1}-\underline{\alpha}_{2}\right]
$$

where $T_{1}$ and $T_{2}$ are that of (49) and (50).

It is worth noting that (54) can be obtained directly from (48) by just reversing the sign. This is to be expected because of the forms of $\underline{\alpha}$ for $\lambda=\lambda_{\min }$ and $\lambda=\lambda_{\max }$ as given in (52) and (53).

In the search for the stability margin of a given injection $\underline{z}_{g}$, of the two feasibility vectors $\underline{\alpha}^{\prime} s$, the one corresponding to the smallest objective function of the form 


$$
D\left(\underline{\alpha} \underline{z}_{g}\right)=\frac{\underline{\alpha}^{\mathrm{T}} \cdot \underline{z}_{g}}{\|\underline{\alpha}\| \underline{z}_{g} \|}
$$

is chosen to compute the gradient function.

\section{3 Algorithms}

The computation of the stability margin can be done using any of unconstrained numerical optimization schemes $[4,5$, 6]. Two methods of the second order type have been considered namely:

(i). The conjugate gradient method due to Fletcher and Reeves $[4,5]$;

(ii) The method of Fletcher and Powell [8,9].

In the initial part of the work, the conjucate gradient method has been tried. Specifically a variation of the algorithm from the SSP' [10] library was used. The conjugate gradient method requires a linear search in each iteration. The number of function evaluations in each iteration depends on the efficiency of the line search. In this application to find the stability margin of an injection, each function evaluation requires the evaluation of the generalized eigenvalue problem. So it is in the interest of. the overall efficiency and economy of computer time to reduce the function evaluations per iteration as well as the number of overall iterations. Presented with this problem, the method of Fletcher 
and Powell $[9,11]$ with approximate linear search seems to offer the likely remedy. It does not only offer the opportunity to reduce the number of function evaluations but the technique also has been claimed $[5,8,9]$ to have better performance and convergence characteristics than the conjugate gradient method. The only possible shortcoming of the method of Fletcher and Powell is the increase in storage necessary to store the matrix $\underline{\mathrm{H}}$, but so far this has not been a serious limitation. The main aspect of the Fletcher and Powell method is the symmetric positive definite matrix $\underline{H}_{i}$, which is updated every operation and guarantees the minimization algorithm to always go "downhill". The direction of search $\underline{s}_{i}$ is of the form

$$
\underline{s}_{i}=-\underline{H}_{i} \underline{g}_{i}
$$

and the step length is

$$
\underline{\delta}_{i}=\rho \underline{S}_{i}=-\rho \underline{H}_{i} \underline{g}_{i}
$$

The matrix $\underline{H}_{i}$ is updated arcording to the following scheme $[8,9]$,

$$
\underline{H}_{i+1}=\underline{H}_{i}+\frac{\delta_{i} \underline{\delta}_{i}^{T}}{\delta_{i}^{T} \underline{y}_{i}}-\frac{\underline{H}_{i} \underline{y}_{i} \underline{y}_{i}^{T} \underline{H}_{i}}{\underline{y}_{i}^{T} \underline{H}_{i} \underline{y}_{i}}
$$

where

$$
\underline{\delta}_{i}=\underline{z}_{1 i+1}-\underline{z}_{I i+1}=\rho \underline{H}_{i} \underline{\delta}_{i}
$$




$$
\begin{aligned}
& \underline{\mathrm{y}}_{\mathrm{i}}=\underline{\mathrm{g}}_{\mathrm{i}+1}-\underline{\mathrm{g}}_{\mathrm{i}} \\
& \underline{\mathrm{H}}_{0}=\mathrm{I}, \text { a unity matrix. }
\end{aligned}
$$

An approximate line search [9], rather than a full line search is used to determine $\rho$, the magnitude of the step length in each iteration. An approximate $\rho *$, or $\delta_{i}^{*}$ is chosen such that a sufficiently large decrease in the objective function is effected in each iteration. In [9], this is done by forcing the change in the objective function $\Delta F$ to be such that

$$
\frac{\Delta \mathrm{F}}{\underline{\mathrm{g}}_{\mathrm{i}}^{\mathrm{T}} \dot{\delta} \mathrm{i}}>\mu
$$

where $0<\mu<1$

The procedure is to find $\underline{\delta}_{i}=-\rho \underline{H}_{i} \underline{g}_{i}$ by trying values $\rho=1, \omega, \omega^{2}, \omega^{3}, \ldots(0<\omega<1)$ which will produce $\delta_{i}^{*}$ such that it satisfies the reduction in objective function as in (61). Fletcher [9] suggested the values $\mu=0.0001$ and $\dot{\omega}=0.1$. Since $\delta_{i}^{*}$ is an approximate solution of the line search, it is important to check on the positive definiteness of the new matrix $\underline{\mathrm{H}}_{\mathrm{i}+1}$ formed from (58). The positive definiteness of $\underline{H}_{i+1}$ can be shown to be true [9], if the inequality $\underline{\delta}_{i}^{T} \underline{y}_{i}>$ o is true. In the version of the algorithm used which is due to University of Waterloo Computing Centre [11], if the approximate line search fails to satisfy the criterion of function reduction and positive definiteness of $\underline{\mathrm{H}}_{i}$, an ordinary 
full line, search is done.

The implementation of the proposed algorithm is illustrated in the flow diagram in Figs. 4.5 and 4.6 . It is necessary to ensure that the matrix $\underline{J}\left(\underline{z}_{0}\right)$ of the generalized eigenvalue problem be positive definite so that we are assured of finite eigenvalues [3]: Geometrically this implies that the feasibility surface $\mathrm{P}_{z 0}$ is closed and bounded. The nature of the vector $\underline{z}_{0}$ is illustrated in the next section.

It has been found that it is only necessary to compute the two extremum eigenvalues once, in the routine. After the first iteration, only either the maximum or the minimum eigenvalue is required. This result is important since it reduces the number of extremum eigenvalue evaluations by almost half.

We haver noted that only the extremum eigenvalues are required. The standard method of computing all the eigenvalues and selecting the maximum and minimum is thus highly ineffective. Of the many techniques which are available to compute extremum eigenvalues, two of Lhell give promising results. They are:

(1) Maximizing and minimizing the Rayleigh quotient $[3,12]$

$$
\lambda=\frac{\underline{x}^{\prime} \underline{I}^{\prime}\left(\underline{z}_{1}\right) \underline{x}}{\underline{x}^{T} \underline{J}\left(\underline{z}_{0}\right) \underline{x}}
$$




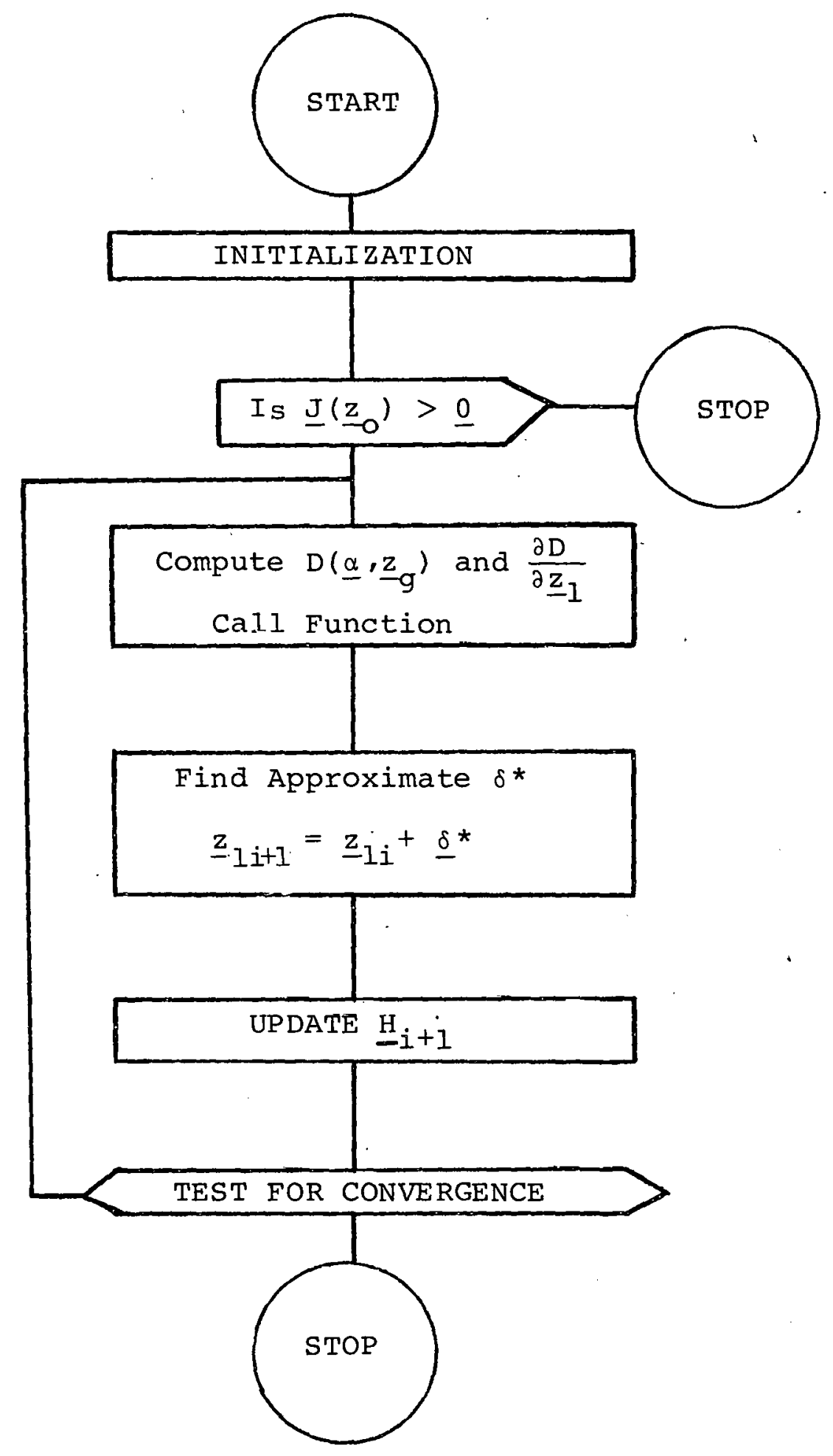

FIGURE 4.5. Main flow diagram to compute stability margin using the method of Fletcher-Powell. 


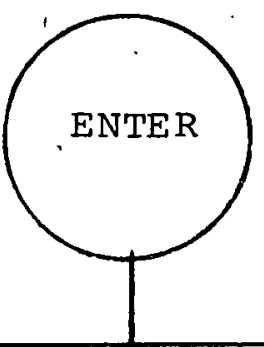

INITIALIZATTIONS

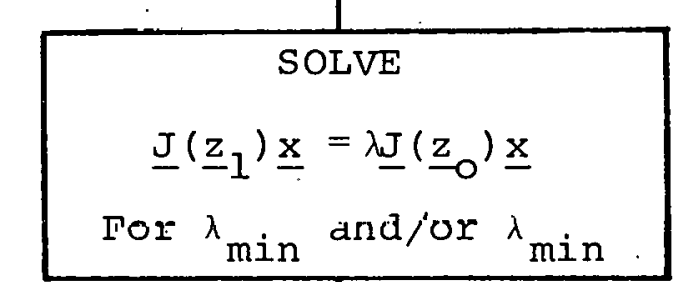

SELECT MINIMUM OF

\begin{tabular}{|c|c|}
\hline \multicolumn{1}{c}{$\mathrm{D}\left(\underline{\alpha}_{,}, \underline{z}_{g}\right)$} \\
\hline COMPUTE & $\frac{\partial \mathrm{D}}{\partial \underline{z}_{1}}$ for \\
$\lambda_{\min }$ or $\lambda_{\max }$ \\
\hline
\end{tabular}

1

1

FIGURE 4.6. Flow diagram of the subroutine to compute the objective function and gradient of the minimization procedure to compute stability margin. 
using some form of numerical optimization scheme like the conjugate gradient method [5], and the method by Fletcher and Powell [8].

(2) The most promising of all is the shifted inverse power approach $[3,12]$. This technique works very well as will be illustrated in the next section. One of the reasons is that the eigenvalues at the end of each iteration decrease monotonically as shown in Fig. 4.9 .

\section{Examples}

The algorithm to compute the stability margin of an injection as developed in the earlier section is tested on 3sample systems, namely,

- A 2-bus transmission line

- A 3-bus network

- Two practical 5-bus networks.

The line diagrams of the sample system are shown in Figs. 4.7, and 4.8 .

Tables $4.0 \mathrm{a}, \mathrm{b}$ summarize the results obtained by applying the algorithm to the 2 and 3 -bus network for.a variety of injections $\underline{z}_{g}$ and bus types. The vector $\underline{\alpha} *$ defines the closest supporting hyperplane or closest feasibility plane, while S.M. $=\mathrm{D}\left(\underline{\alpha}^{*}, \underline{z}_{\mathrm{g}}\right)$ is the stability margin. The injections which have negative stability margin result in non-convergence 


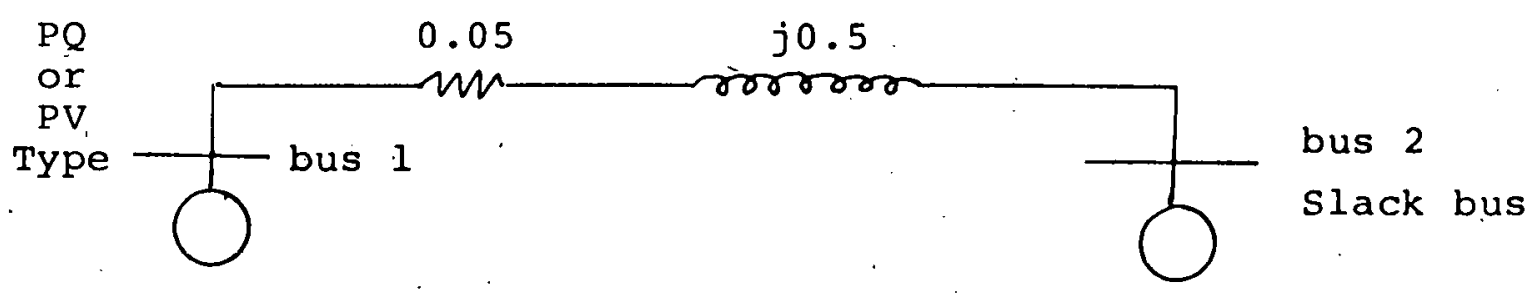

(a)

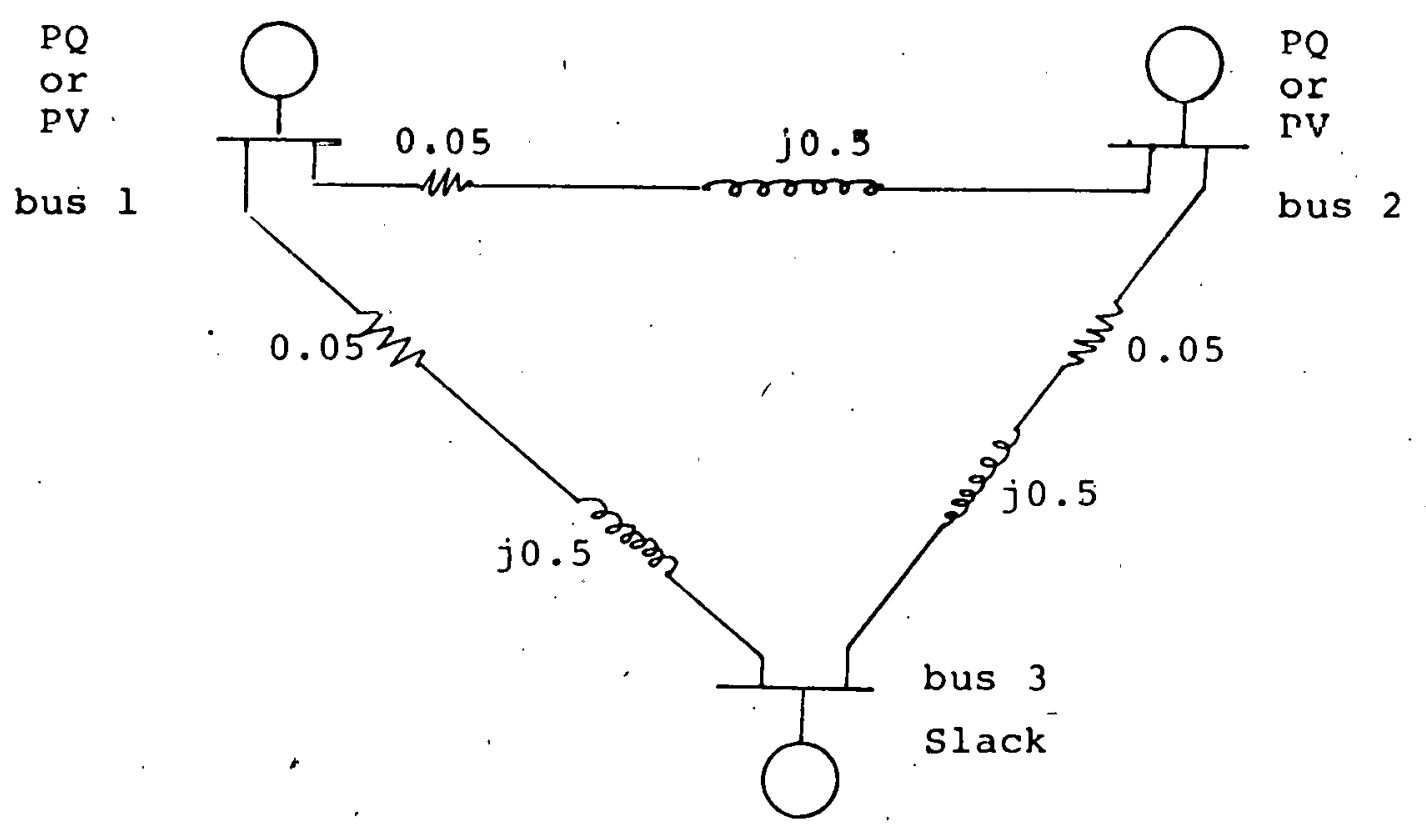

(b)

FIGURE 4.7. (a) Transmission Line

(b) A 3-bus network 


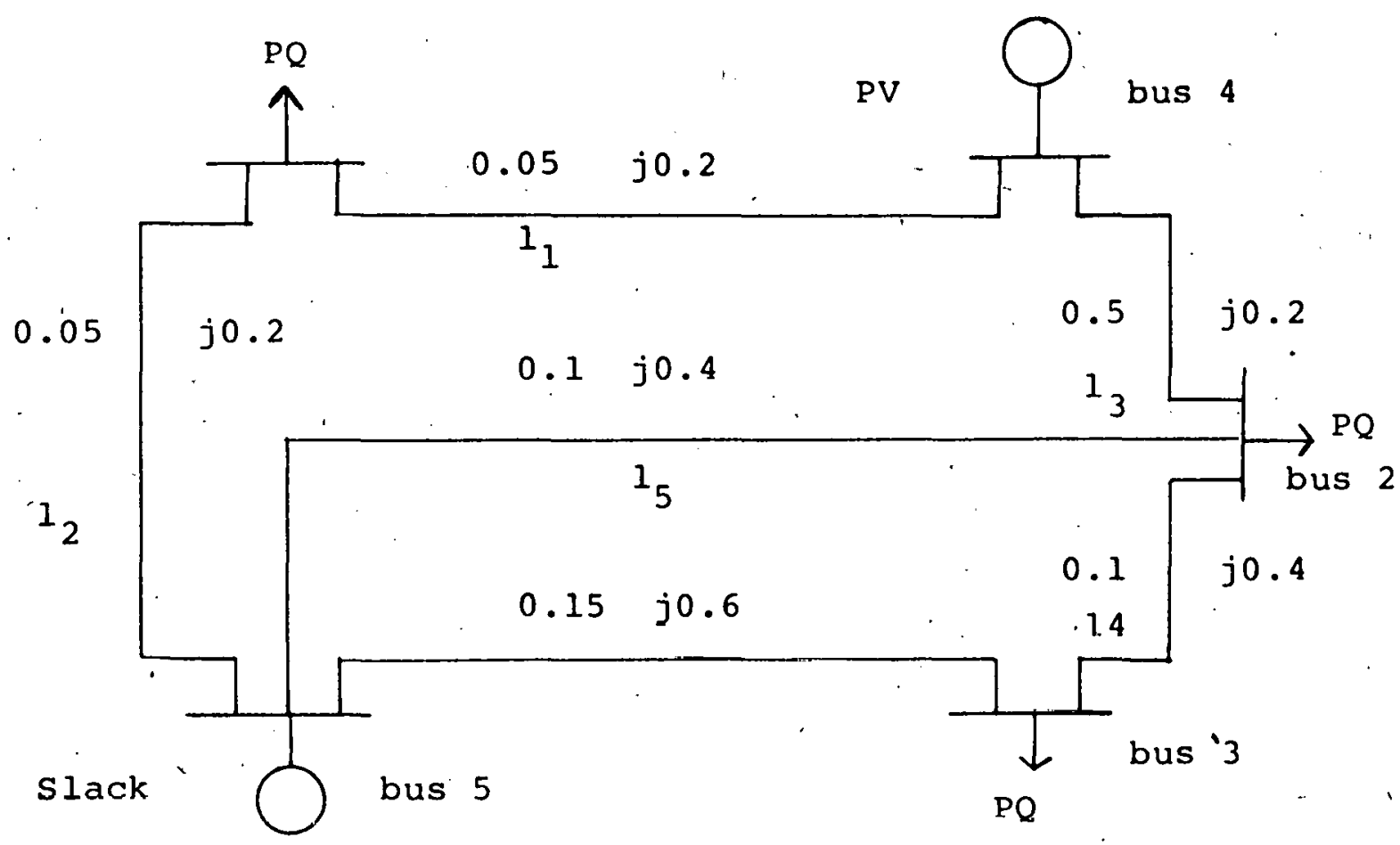

$1_{1}, 1_{2}, 1_{3}, 1_{4}, 1_{5}, 1_{6}$ are line numbers. 100 Mva base.

FIGURE 4.8. 5-bus network from Stevenson. 
of the load flow. The injection, for a particular system configuration, which has greater stability margin, S.M. is more stable steady statewise. For instance, for the 3-bus network with 2-PQ buses, the injection $\underline{\mathrm{z}}_{\mathrm{g}}=(0.3,0.2,0.3,-0.1,1.0)$ is "more" stable than the injection $\underline{z}_{g}=(0,-0.1,0,-0.2$, 1.0).

It is noted earlier that it is important that $\underline{z}_{0}$ be chosen such that the matrix $\underline{J}\left(\underline{z}_{0}\right)$ is positive definite for the eigenvalues to be finite [3]. Through the many runs that have been madc, if the inpedance of the lines of the network are normalized and that the reactances are greater than $0.1 \mathrm{p} . \mathrm{u}$. , the following choice of $\underline{z}_{0}$ is satisfactory:

$$
\begin{aligned}
& \text { (a) For a PQ bus, set } \\
& z_{0}(P)=0 \\
& z_{O}(Q)=0.1
\end{aligned}
$$

where $z_{0}(P)$ implies the elements of $z_{0}$ corresponding to the real power $\mathrm{P}$.

(b) For a PV bus, set

$$
\begin{aligned}
& z_{0}(P)=0 \\
& z_{0}\left(V^{2}\right)-1.0
\end{aligned}
$$

(c) For a slack bus, set

$$
z_{0}\left(v^{2}\right)=1.0
$$


TABEE $4.07(\mathrm{a})$

Summary of Result's for the stability Margin of A Variety of $\underline{z}_{g}$ for 2-bus Network

\begin{tabular}{|c|c|c|c|c|}
\hline $\begin{array}{c}\text { Given Injection } \\
\underline{z}_{\mathrm{g}}\end{array}$ & $\begin{array}{c}\text { Optimal Search } \\
\text { Direction } \\
\underline{z}_{1}^{*}\end{array}$ & $\begin{array}{c}\text { 2losest Boundary } \\
\text { Injection } \\
\underline{z}_{\mathrm{b}}^{\star}\end{array}$ & $\begin{array}{l}\text { Closest Hyperplane } \\
\text { Defined by } \underline{\alpha}^{* \mathrm{~T}_{z}=0}\end{array}$ & $\begin{array}{c}\text { Stability } \\
\text { Margin } \\
\text { S.M. }=\mathrm{D}\left(\underline{\alpha}^{*}, \underline{z}\right)\end{array}$ \\
\hline $0.0,-0.25,1.0$ & $0.0,2.01,-0.10$ & $0.0,-0.5,1.0$ & $0.0,2.13,1.07$ & 0.217 \\
\hline $0.5,-0.5,1.0$ & $-5.6,13.7,-1.32$ & $0.46,0.43,1.0$ & $-5.6,14.7,8.9$ & -0.058 \\
\hline$-0.25,0.25,1.0$ & $0.85,0.8,-0.03$ & $-0.804,-0.12,1.0$ & $0.85,0.89,0.79$ & 0.514 \\
\hline
\end{tabular}

(a)

\begin{tabular}{rlccc}
$1.0,1.0,1.0$ & $1.5,0.5,0.5$ & $2.0,1.0,1.0$ & $1.5,1.5,1.5$ & 0.333 \\
$3.0,1.0,1.0$ & $1.63,0.36,0.64$ & $2.2,1.01,0.98$ & $-1.63,1.9,1.65$ & -0.133 \\
$-0.5,1.0,1.0$ & $2.86,-0.83,1.8$ & $-1.2,1.01,0.49$ & $2.86,1.41,4.08$ & 0.524 \\
\hline
\end{tabular}

(b)

(a) $\underline{z}_{0}=(0,0.1,1.0)$, bus 1 is a $P Q$ bus, bus 2 is a slack bus, $\underline{z}=\left(P_{1}, Q_{1}, V_{2}^{2}\right)$

(b) $\quad \underline{z}_{0}=(0,1.0,1.0)$, bus 1 is.a.PV bus, bus 2 is a slack bus, $\underline{z}^{\prime}=\left(P_{1}, v_{1}^{2}, v_{2}^{2}\right)$ 
TABLE $4.0(\mathrm{~b})$

Summary of Results for the Stability Margin of Variety of $z_{g}$, for a 3-Bus Network

\begin{tabular}{|c|c|c|c|c|}
\hline $\begin{array}{c}\text { Given Injection } \\
\frac{z}{-g}\end{array}$ & $\begin{array}{c}\text { Optimal Search } \\
\text { Direction } \\
\underline{z}_{1}^{*} \\
\end{array}$ & $\begin{array}{c}\text { Closest Boundary } \\
\text { Injection } \\
\underline{\mathrm{z}}_{\mathrm{b}}^{\star}\end{array}$ & $\begin{array}{l}\text { Closest Hyperplane } \\
\text { Lefined } \mathrm{By}^{*} \underline{\alpha}^{*} \underline{T}_{2}=0 \\
\underline{\alpha}^{*}\end{array}$ & $\begin{array}{c}\text { Stability Margin } \\
\text { S.M. }=D\left(\underline{\alpha}^{*}, \underline{z}_{g}\right)\end{array}$ \\
\hline
\end{tabular}

(a) $\underline{z}=\left(P_{1}, Q_{1}, P_{2}, Q_{2}, v_{3}^{2}\right)$

\begin{tabular}{|c|c|c|c|c|}
\hline $\begin{array}{r}5.0,1.0,3.0,1.0,1.0 \\
1.0,1.0,-1.0,1.0,1.0 \\
-1.2,1.0,-0.8,1.0,1.0\end{array}$ & $\begin{array}{c}1.1,0.44,0.94,0.58,-0.02 \\
-0.01,-4.6,2.4,1.0,-4.5 \\
1.4,-0.54,1.3,-0.59,2.1\end{array}$ & $\begin{array}{l}2.6,0.89,1.5,0.85,1.0 \\
0.79,1.0,-1.0,0.68,0.86 \\
-1.6,1,-1.1,1,0.59\end{array}$ & $\begin{array}{c}-1.1,1.4,-0.94,1.2,1.8 \\
-0.01,0.66,2.4,1.5,0.71 \\
1.4,0.78,1.3,0.74,3.5\end{array}$ & $\begin{array}{r}-0.205 \\
0.412 \\
0.2 .49\end{array}$ \\
\hline
\end{tabular}

(b) $\underline{z}=\left(P_{1}, v_{1}^{2}, P_{2}, v_{2}^{2}, v_{3}^{2}\right.$

\begin{tabular}{|c|c|c|c|c|}
\hline $\begin{array}{l}2.3,3.0,2.5,1.0,1.0 \\
0.5,-0.5,1.2,1.0,1.0 \\
-0.6,0.4,-0.8,1.0,1.0\end{array}$ & $\begin{array}{r}4.9,-0.64,5.3,1.9,-0.54 \\
1.1,-2.5,0.51,0.56,0.64 \\
-0.95,-0.21,-0.73,1.2,-0.05\end{array}$ & $\begin{array}{l}1.4,2.2,1.8,0.57,0.69 \\
0.66,-0.8,1.4,1,1 \\
-0.2,0.41,-1.3,1.1,0.67\end{array}$ & $\begin{array}{l}-4.9,1.7,-5.3,0.8,1.1 \\
-1.1,2.7,-0.51,1.9,1.8 \\
0.96,0.39,0.73,0.6,1.8\end{array}$ & $\begin{array}{l}0.008 \\
0.141 \\
0.359\end{array}$ \\
\hline
\end{tabular}

(c) $\underline{z}=\left(P_{1}, Q_{1}, P_{2}, v_{2}^{2}, v_{3}^{2}\right.$

(a) $\underline{z}_{0}=(0,0.1,0,0.1,1)$ Bus 1 and bus 2 are $P Q$ buses, Bus 3 always kept as slack bus.

(b) $\underline{z}_{0}=(0,1,0,1,1) \quad$ Bus 1 and bus 2 are PV buses.

(c) $z_{0}=(0,0.1,0,1,1)$ Bus 1 is $P Q$ bus, bus 2 is PV bus. 
A more realistic sample system is the 5-bus system from Stevenson [13]. It has 3-PQ buses and 2-generator buses, one of which is taken as the slack bus as shown in Fig. 4.8.

A feasible operating point is taken as the basis for this illustration and has the form

$$
\underline{z}_{g}=[-0.6,-0.2,-0.6,-0.3,-0.4,-0.1,1.0,1.0,1.0]
$$

where

$$
\underline{z}=\left[P_{1}, Q_{1}, P_{2}, Q_{2}, P_{3}, Q_{3}, P_{4}, V_{4}^{2}, V_{5}^{2}\right]
$$

The load flow solution of $\underline{z}_{g}$ is given in Table 4.1 and 4.2 . The bus voltages lie between 0.9 and 1.0 which can be considered quite acceptable and secure.

The summary of the pertinent results using the given injection above is shown in Tables $4.3,4.4$ and 4.5 . The stability margin of the injection $\underline{z}_{g}$ is U.215, a pusilive valuo, since there exist a load flow solution as shown in Tables 4.1 and 4.2 .

The closest boundary injection $\underline{z}_{b}$ represents the closest injection on the boundary of $R_{z}$ to the operating point $\underline{z}_{g}$. Suppose we:define an incremental vector such that

$$
\underline{u}_{g}=\underline{z}_{b}-\underline{z}_{g}
$$

Thus incremental injection $\Delta \underline{z}$ represents the fastest way or the shortest path in which the operating point $\underline{z}_{g}$ will reach 
TABLE 4.1

Load Flow Solution of the Secure Operating Point $\underline{z}_{g}$

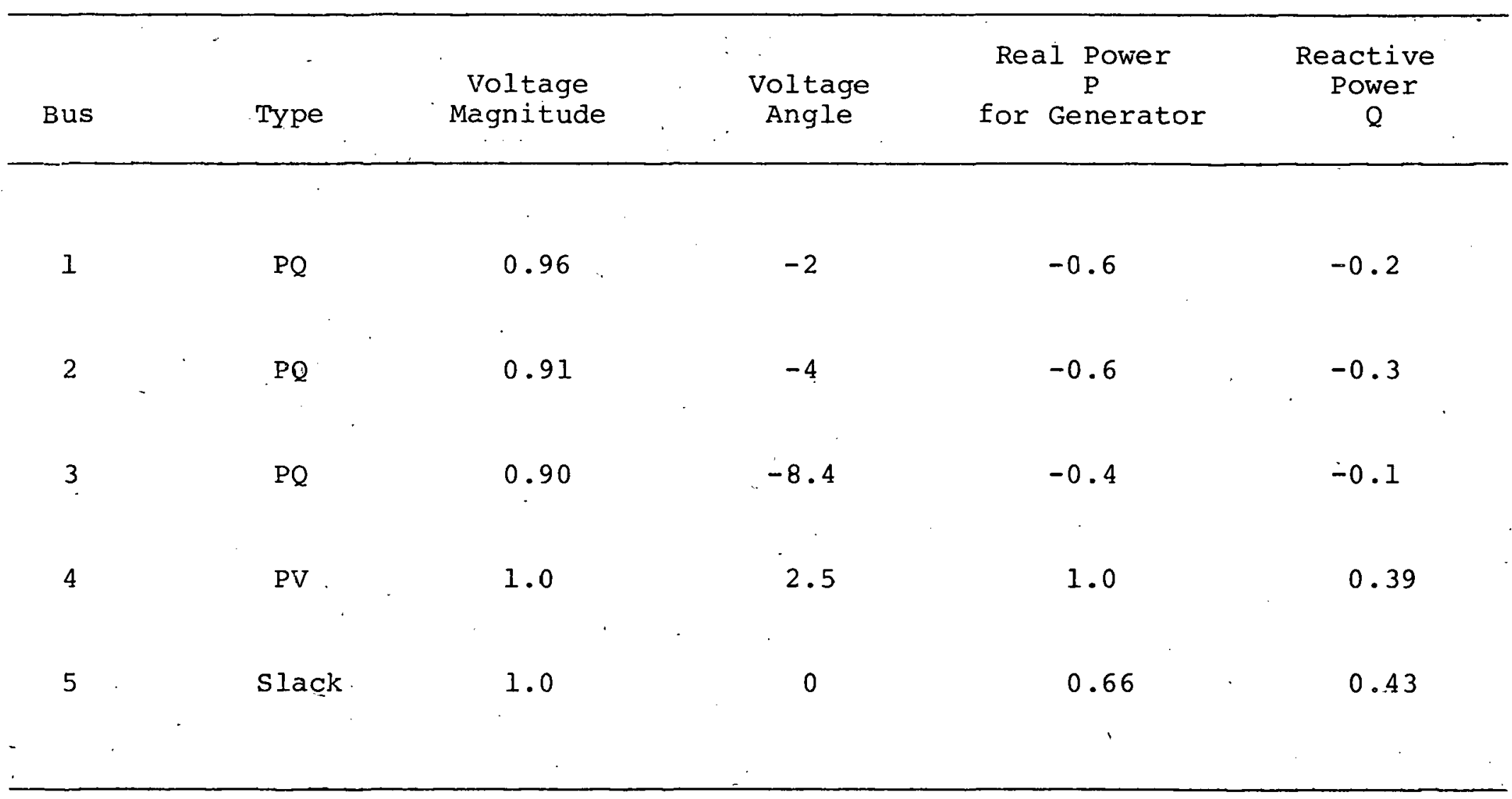


TABLE 4.2

Line Flows

\begin{tabular}{rrrrr}
\hline Line & From & To & Real Power & Reactive Power \\
\hline 1 & 1 & 4 & -0.398 & -0.069 \\
1 & 4 & 1 & 0.407 & 0.105 \\
2 & 1 & 5 & -0.202 & -0.130 \\
2 & 5 & 1 & 0.205 & 0.143 \\
3 & 2 & 4 & -0.572 & -0.197 \\
3 & 4 & 2 & 0.593 & 0.284 \\
4 & 2 & 3 & 0.165 & 0.028 \\
4 & 3 & 2 & -0.161 & -0.015 \\
5 & 2 & 5 & -0.193 & -0.131 \\
5 & 5 & 2 & 0.200 & 0.157 \\
6 & 3 & 5 & -0.239 & -0.085 \\
6 & 5 & 3 & 0.251 & 0.133 \\
\hline
\end{tabular}


TABLE 4.3

Summary of the Feasibility Condition $\circ \equiv$ the Given Operating Secure Point $\underline{z}_{g}$ of 5 Bus-system from stevenson

\section{GENERAL RESULT}

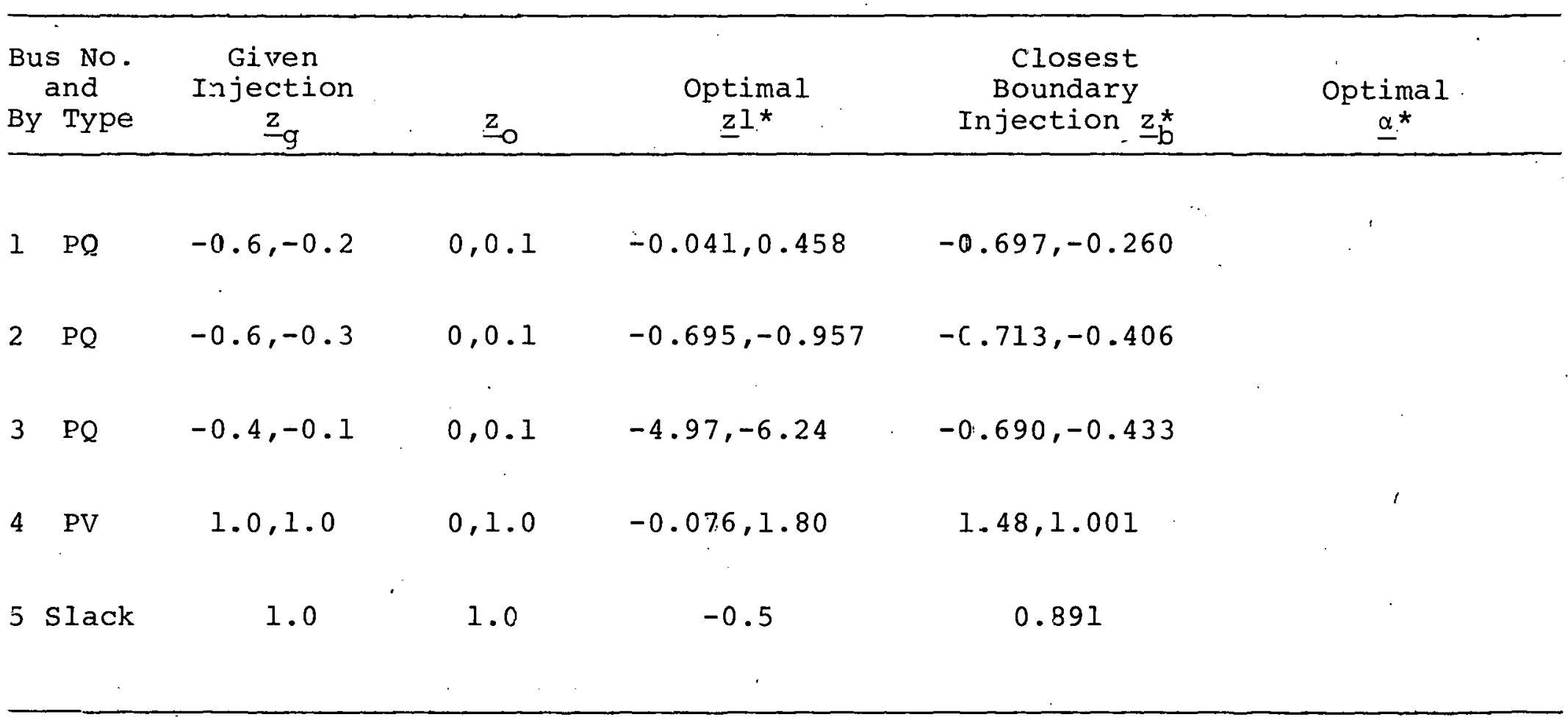


TABLE 4.4

Details of Closest Boundary Injection

\begin{tabular}{|c|c|c|c|c|c|}
\hline Bus & Voltage & $\begin{array}{l}\text { Voltage } \\
\text { Magnitude }\end{array}$ & $\begin{array}{l}\text { Voltage } \\
\text { Angle }\end{array}$ & Real Power. & $\begin{array}{c}\text { Reactive } \\
\text { Power }\end{array}$ \\
\hline 1 & $0.921-j 0.075$ & 0.924 & -4.62 & -0.659 & -0.260 \\
\hline 2 & $0.759-j 0.132$ & 0.771 & -9.85 & -0.713 & -0.406 \\
\hline 3 & $0.447-j 0.210$ & 0.494 & -25.00 & -0.690 & -0.433 \\
\hline 4 & $1.0-j 0.02$ & 1.006 & -1.619 & 1.118 & 1.300 \\
\hline 5 & $0.944-j 0$ & 0.944 & 0 & 1.247 & 1.015 \\
\hline
\end{tabular}


TABLE 4.5

Line Flows of Boundary Injection $\underline{z}_{b}^{*}$

\begin{tabular}{crrrr}
\hline Line & From & To & Real Power & Reactive Power \\
\hline 1 & 1 & 4 & -0.310 & -0.269 \\
1 & 4 & 1 & 0.320 & 0.308 \\
$\dot{2}$ & 1 & 5 & -0.349 & 0.009 \\
2 & 5 & 1 & 0.357 & 0.019 \\
3 & 2 & 4 & -0.718 & -0.666 \\
3 & 4 & 2 & 0.799 & 0.988 \\
4 & 2 & 3 & 0.370 & 0.475 \\
4 & 3 & 2 & -0.304 & -0.231 \\
5 & 2 & 5 & -0.365 & -0.216 \\
5 & 5 & 2 & 0.395 & 0.337 \\
6 & 3 & 5 & -10.381 & -0.202 \\
6 & 5 & 3 & 0.495 & 0.659. \\
\end{tabular}


unfeasibility or will lose steady state stability. As shown in Table 4.3, this change in $\underline{z}_{g}$ is not too unreasonable and may well take place in practice. For bus 1 for instance, the example entailsan increase of real load by $23 \%$ and an increase of reactive load by $40 \%$. One would expect a smaller change in injection $\Delta \underline{z}_{g}$ to lose feasibility if the system is fully loaded.

It is also interesting to observe that at the boundary of the feasibility region the bus voltage magnitudes are not very far off the nominal value of 1 p.u. As shown in Table 4. 4 the voltages at buses 1,4 and 5 are within the tolerance of $\pm 10 \%$

The voltage angles between the lines are less than $25^{\circ}$ (Table 4.4). This is an important observation since this implies that one can lose steady state stability without having the angles across, the lines exceed $25^{n}$.

It is also worth noting that the real and reactive power injection of theslack bus, and the reactive power injection of the generator at bus 4 of the closest boundary injection as shown in Table 4.4 , corresponds to normal generator over excited conditions. There need not be abnormal generator conditions before one can lose steady state stability. With all the injections at all the buses for the closest boundary injection $\underline{z}_{\mathrm{b}}$ to be quite nominal, one would expect the corresponding line flows to be of reasonable values. This is well illustrated by comparing the line flows for $\underline{z}_{b}$ 
of Table 4.5 and line flows of the secure operating point $\underline{z}_{g}$. in Table 4.2 .

Another practical 5-bus system from Stagg and El-Abiad [14] has also been used to gain numerical experience with the proposed stability algorithm. This 5-bus network is also used to illustrate the concept of constrained closest boundary injection in the next section. The details of this network are described in the next sub-section. In order to make the illustration complete, an unfeasible injection $\underline{z}_{g}$ is taken and is of the form

$$
\underline{z}_{g}=(-1.5,-1.5,-1.5,-1.5,-1.5,-1.5,1.5,1.0,1.0)
$$

where

$$
\underline{z}=\left(P_{1}, Q_{1}, P_{2}, Q_{2}, P_{3}, Q_{3}, P_{4}, V_{4}, V_{5}{ }^{2}\right)
$$

The stability margin of the injection $\underline{z}_{g}$ is -0.022 , which is unfeasible. If a load flow is run using the above injection $\underline{z}_{y}$, no convergence can bc obtained. The details pertaining to the other aspects of the result is summarized in Tables 4.6 and 4.7 .

Up to this juncture, no mention has been made on the initial constant vector $\underline{z}_{1}$ used. For the 5-bus sample system, initial $\underline{z}_{1}$ of the form

$$
\underline{z}_{1}=\underline{z}_{g}-z_{0}
$$

and 
TABLE 4.6

Summary of the Feasibility Condition of An Injection $\underline{z}$

of a 5-Bus system from Stagg and El-Abiad

\section{GENERAL RESULT}

\begin{tabular}{|c|c|c|c|c|c|}
\hline $\begin{array}{l}\text { Bus No. } \\
\text { and } \\
\text { By Type }\end{array}$ & $\begin{array}{c}\text { Given } \\
\text { Injection } \\
\underline{z}_{g} .\end{array}$ & $\underline{z}$ & $\begin{array}{l}\text { Optimal } \\
\underline{z} I^{*}\end{array}$ & $\begin{array}{c}\text { Closest } \\
\text { Boundary } \\
\text { Injection, } \underline{z}_{b}^{*}\end{array}$ & $\begin{array}{l}\text { Optimal } \\
\underline{\alpha}^{*}\end{array}$ \\
\hline & . & & & & \\
\hline $1 \mathrm{PQ}$ & $-1.5,-1.5$ & $0.0,0.1$ & $-1.56 .8,-1.334$ & $-1.376,-1.371$ & $1.568,2.568$ \\
\hline$P Q$ & $-1.5,-1.5$ & $0.0,0: 1$ & $-2.477,-2.665$ & $-1.372,-1.365$ & $2.477,3.899$ \\
\hline$P Q$ & $-1.5,-1.5$ & $0.0,0.1$ & $-2.153,-2.325$ & $-1.373,-1.367$ & $2.153,3.559$ \\
\hline $\mathrm{PQ}$ & $-1.5,-1.5$ & $0.0,0.1$ & $0.015,7.934$ & $1.383,0.941$ & $-0.015,4.409$ \\
\hline 5 slack & 1.0 & 1.0 & -5.791 & i. 0 & 18.094 \\
\hline
\end{tabular}


TABLE 4.7

Details of Closest Boundary Injection

\begin{tabular}{|c|c|c|c|c|c|}
\hline Bus & Voltage & $\begin{array}{l}\text { Voltage } \\
\text { Magnitude }\end{array}$ & $\begin{array}{l}\text { Voltage } \\
\text { Angle }\end{array}$ & Real. Power. & $\begin{array}{c}\text { Reactive } \\
\text { Power }\end{array}$ \\
\hline 1 & $0.537-j 0.115$ & 0.549 & -12.1 & -1.38 & -1.37 \\
\hline \multirow[t]{2}{*}{2} & $0.475-j 0.119$ & 0.489 & -14.1 & -1.37 & -1.37 \\
\hline & & $\cdot$ & $i$ & . & \\
\hline 3 & $0.501-j 0.112$ & 0.514 & -12.6 & -1.37 & -1.37 \\
\hline-4 & $0.469+j 0.032$ & 0.970 & 1.9 & 1.38 & 0.95 \\
\hline 5 & $1.0+j 0$ & 1.0 & 0.0 & 4.55 & $8: 49$ \\
\hline & & & & & : \\
\hline
\end{tabular}




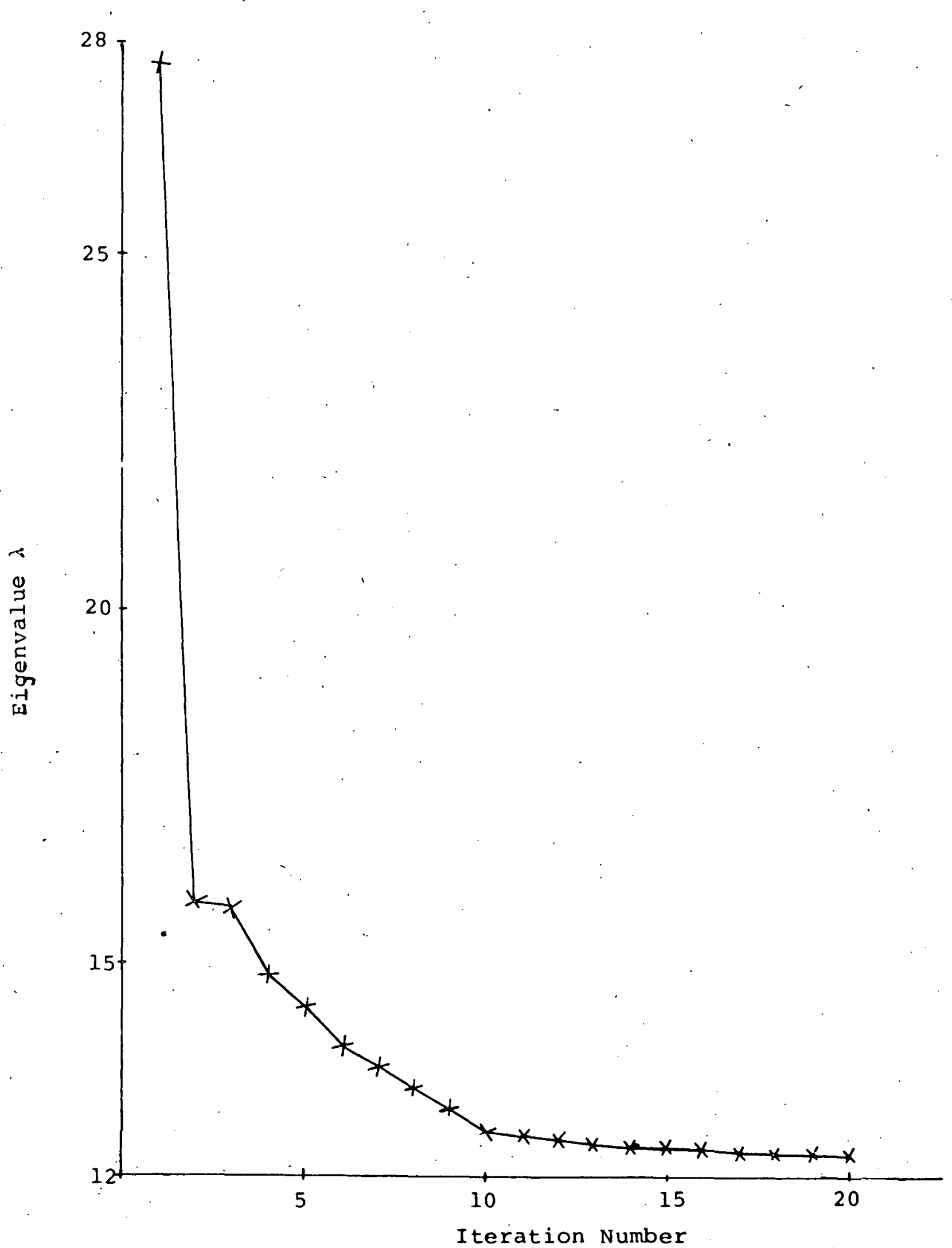

FIGURE 4.9. Graph of eigenvalue versus iteration number. 


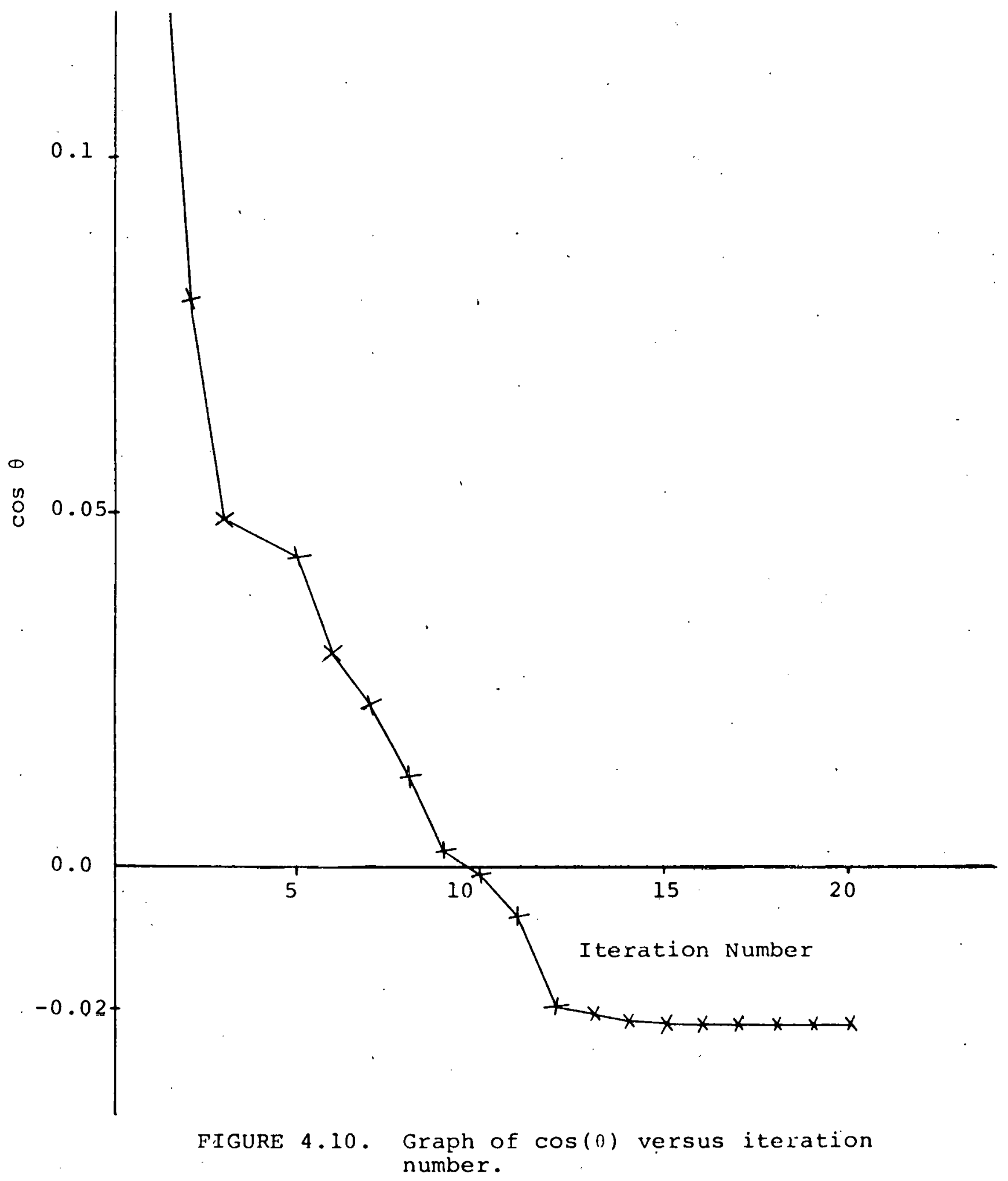




$$
\underline{z}_{1}=\underline{z}_{g}
$$

have been found to be satisfactory.

In each iteration of the optimization scheme to evaluate the stability margin of $\underline{z}_{g}$, it is necessary to compute the objective function $\underline{\alpha}^{\mathrm{T}} \underline{\underline{z}}_{g} /\|\underline{\alpha}\|\left\|\underline{z}_{g}\right\|$ and it requires one extreme eigenvalue of $\underline{\mathrm{J}}\left(\underline{z}_{1}\right) \underline{x}=\lambda \underline{\mathrm{J}}\left(\underline{z}_{0}\right) \underline{x}$. As illustrated in Fig. 4.9, the eigenvalue decreases monotically towards a minimum value. The objective function which is the cosine of the angle between $\underline{\alpha}^{T}$ and $\underline{z}_{g}$ behaves in a similar fashion as shown in Fig. 4.10. The convergence is fast. After nine iterations, (the optimization problem has nine variables for a 5buis system); the objective function has come within more than $90 \%$ of the solution.

It has been mentioned earlier that the method of computing all the eigenvalues and choosing the required extremum is inefficient. It is also noted that after essentially the first iteration, either the maximum or the minimum eigenvalue is only required. Besides the standard method, two other techniques were investigated, namely:

- the shifted inverse method

and

- maximizing and/or minimizing the Rayleigh quotient

$$
\lambda=\frac{\underline{x}^{T} \underline{J}\left(\underline{z}_{1}\right) \underline{x}}{\underline{x}^{T} \underline{J}\left(\underline{z}_{0}\right) \underline{x}}
$$


TABLE 4.8

Stability Margin and Relevant Eigenvalue As a Function of Iteration Number

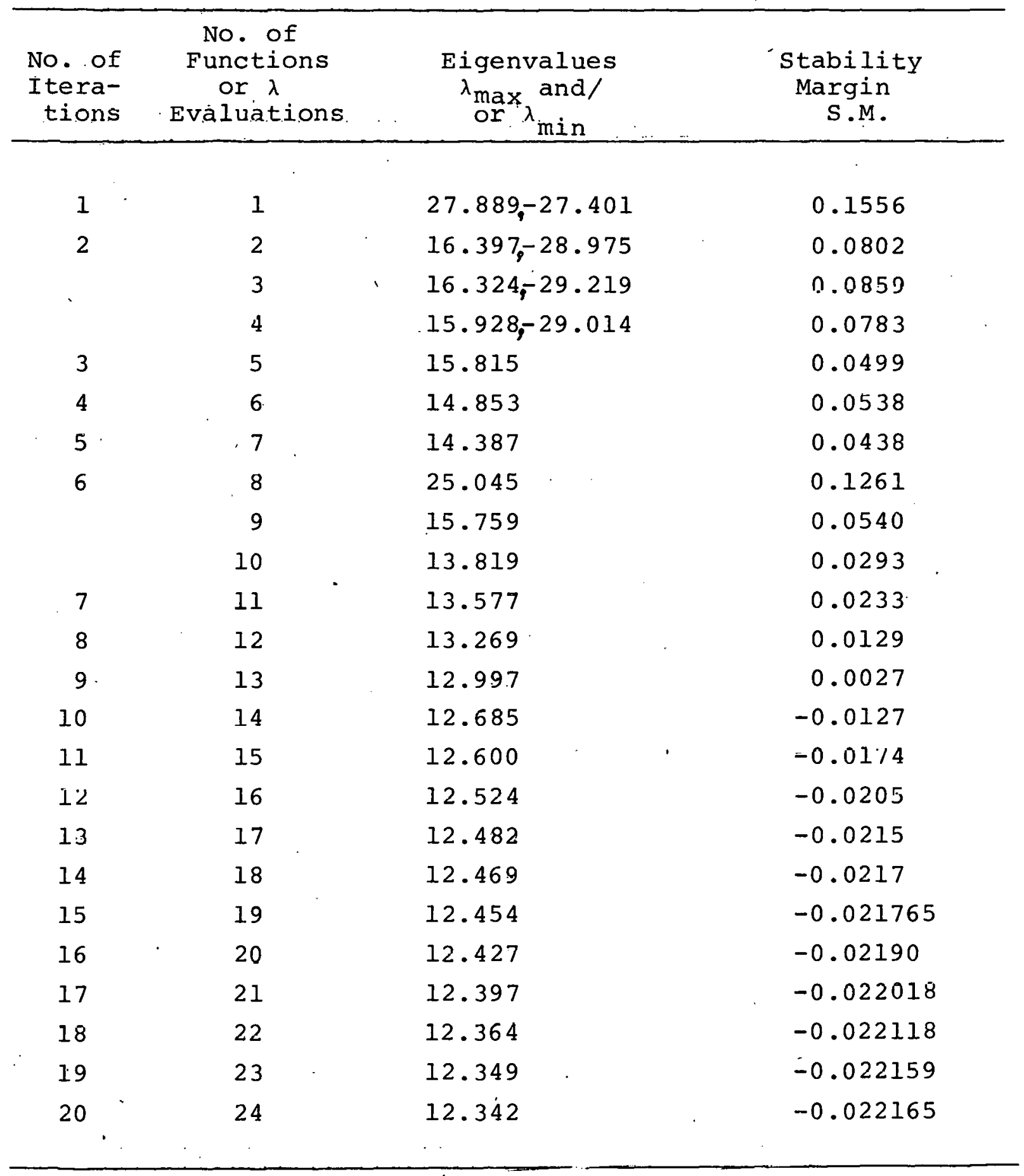


TABLE 4.9

Comparison of Performance of the Techniques to Compute Stability Margin for the Three Different Methods of Computing Eigenvalues

\begin{tabular}{|c|c|c|c|}
\hline & $\begin{array}{l}\text { Standard } \\
\text { Method. }\end{array}$ & $\begin{array}{l}\text { Shifted Inverse } \\
\text { Power } \\
\text { Method }\end{array}$ & $\begin{array}{l}\text { Rayleigh } \\
\text { Quotient } \\
\text { Method }\end{array}$ \\
\hline No. of Iterations & 20 & 20 & 20 \\
\hline $\begin{array}{l}\text { No. of Function } \\
\text { Evaluations }\end{array}$ & 24 & 24 & 24 \\
\hline $\begin{array}{l}\text { Computing rime To } \\
\text { Compute All Eigen- } \\
\text { values in CPU sec. }\end{array}$ & $7.20 \mathrm{sec}$ & $2.42 \mathrm{sec}$ & $4.50 \mathrm{sec}$. \\
\hline $\begin{array}{l}\text { Total Computing } \\
\text { Time To Evaluate } \\
\text { Stability Margin }\end{array}$ & 8.89 & $3.60 \mathrm{sec}$ & $6.18 \mathrm{sec}$. \\
\hline
\end{tabular}


The summary of the computing time using IBM $370 / 158$. for the three techniques is shown in Table 4.9. The effort to evaluate the eigenvalues represent a very substantial part of the overall computing time. For the standard method which computes ali the eigenvalues, the eigenvalue evaluation uses $81 \%$ of the total CPU time. Of the three techniques, the shifted inverse technique is by far the best. The computing effort to evaluate the eigenvalues reduces from $7.20 \mathrm{sec}$ to $2.42 \mathrm{sec}$, an improvement of almost 3 times. The overall computing time reduces by about a similar margin. The method which computes the eigenvalue by maximizing and/or minimizing the Rayleigh quotient gives some improvement in the overall performance but not as good as the method which uses the shifted inverse technique.

\subsection{Sufficient Feasibility Set}

In the previous section, a technique to compute the stability margin of an injection is developed. This result can be extended and generalized to characterize a set $R_{S} \subset R_{z}$ defining a sufficient condition for steady state stability.

The stability margin of an injection as defined earlier. is of the form

$$
\text { S.M. }=\frac{\underline{\alpha}^{\mathrm{T}} \underline{z}_{\mathrm{g}}}{\|\underline{\alpha}\| \underline{\alpha} \| \underline{\|}}
$$

where $\underline{\alpha}=\underline{\alpha}^{*}$, the normal vector defining the closest 

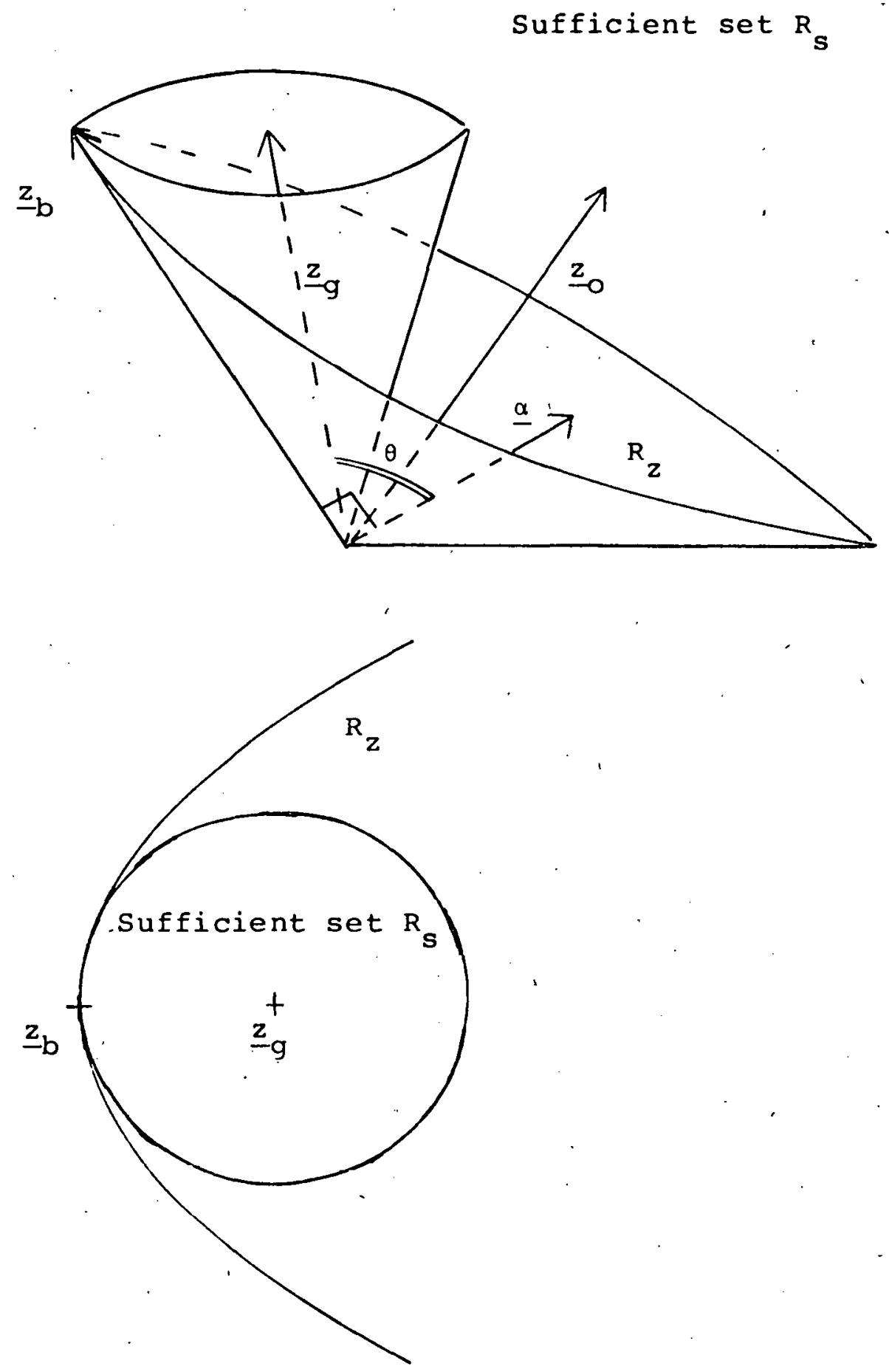

FIGURE 4.11. Sufficient set of a given $\underline{z}_{g}^{\prime}$ for a 2 -bus 
supporting hyperplane or feasibility plane.

Suppose a feasible injection $\underline{z}_{g} \varepsilon R_{z}$ is chosen, then its stability margin S.M. will be positive and a closest boundary injection $\underline{z}_{b}$ will be defined as illustrated in Fig. 4.11. Since $\underline{z}_{b}$ is closest to $\underline{z}_{g}$ in the sense that the angle between them is minimum, and the feasibility region $R_{z}$ is convex, then any injection which has an angle less than the minimum angle must be in $R_{z}$.

The cosine of the angle between $\underline{z}_{g}$ and $\underline{z}_{b}$ in terms of. the stability margin has the form.

$$
\frac{\underline{z}_{b}^{\mathrm{T}} \underline{z}_{g}}{\left\|\underline{z}_{b}\right\| \underline{z}_{g} \|}=\left[1 .- \text { S.M. }{ }^{2}\right]^{\frac{1}{2}}
$$

which is immediate from the geometry in Fig. 4.11. A circular conical sufficiency set can now be defined such that

$$
\mathrm{R}_{S}=\left\{\underline{z} \mid \frac{\underline{z}^{\mathrm{T}} \underline{z}-g}{\|\underline{z}\|\left\|\underline{z}_{g}\right\|} \geqslant\left[1-\mathrm{S} \cdot \mathrm{M}^{2}\right]^{\frac{1}{2}} ; \underline{z}_{g} \varepsilon \mathrm{R}_{\mathrm{z}}\right\}
$$

If a sufficiency set $R_{S}$ is available the steady state stability of an operating point $\underline{z}$ can be easily determined by checking if it belongs to the sufficiency set $R_{S}$. This characterization suggests a possible applicatiun in security monitoring and enhancement.

A continuing research effort is focused on "enlarging" the sufficient set such that a largest possible circular cone can be defined for a feasibility region $R_{z}$. 
4.5 Closest Boundary Injection with Equality Constraints

The viability of the technique to compute the stability margin and the closest boundary injection of an injection vector has been demonstrated in the previous section for arbitrary variations in $\underline{z}$.

For some applications such as security control and monitoring, one may want to find the set of changes in the power generation alone, for which we may lose feasibility or steady state stability. It may also. be required, for example, to find the closest boundary injection such that the real and reactive powers of the load buses are in some specified linear proportion, such as having a constant'power factor.

Suppose that the load at bus 1 is required to remain fixed at 0.5 P.U. of real power and 0.3 P.U. of reactive power. This implies that the operating point $\underline{z}_{g}$ and the closest boundary injectiui $\underline{\Sigma}_{b}^{*}$ with fixed injections at bus 2 , must lie on the planes

$$
(1, \underline{0})^{\mathrm{T}} \underline{z}=0.5
$$

and

$$
(0,1, \underline{0})^{T} \underline{z}_{z}=0.3
$$

Similarly at bus 2 , it may be required that the boundary injection $\underline{z}_{b}^{*}$ be such that

$$
\mathrm{Q}_{2}=0.6 \mathrm{P}_{2}
$$


which means the boundary injection $\underline{z}_{b}^{*}$ must lie on the plane

$$
(0,0,-0.6,1, \underline{0})^{\mathrm{T}} \underline{\mathrm{z}}=0
$$

These additional conditions that are imposed on the boundary injection can be viewed as a constraint requiring the boundary injection to lie on a subset or an affine manifold [15] defined by the intersection of the planes of the form $\underline{B}_{i}^{\mathrm{T}} \underline{z}=\mathrm{k}_{\mathrm{i}}$ as in (72), 73), (74), and (75). We can define this affine manifold as

$$
\Omega=\bigcap_{i=1}^{\mathrm{m}} \underline{\beta}_{i}^{\mathrm{T}} \underline{z}=\mathrm{k}_{i}
$$

where $m$ is the number of constraints.

More formally then, the evaluation of the stability margin and closest boundary injection corresponds to the following optimization problem:

$$
\min _{\underline{z}_{1}} \frac{\underline{\alpha}^{\mathrm{T}} \cdot \underline{z}_{g}}{\|\underline{\alpha}\| \underline{z}_{g} \|}
$$

subject to

$$
\underline{R}_{i}^{\mathrm{T}} \underline{\mathrm{z}}_{\mathrm{b}}=k_{\mathrm{i}} \quad \mathrm{i}=1, \ldots, \mathrm{m}
$$

The solution of this optimization gives the constrained stability margin, S.M., and the closest boundary injection $z_{b}^{*}$ such that 


$$
\underline{\mathrm{z}}_{\mathrm{b}}^{*} \varepsilon \Omega
$$

and also

$$
\underline{z}_{b}^{*} \varepsilon \quad\left[\operatorname{Ext}\left(R_{z}\right) \cap \Omega\right]
$$

where $\operatorname{Ext}\left(R_{z}\right)$ is the boundary of the feasibility region.

A geometrical illustration of the affine manifold and constraint closest boundary injection is shown in Fig. 4.12.

One can of course solve the optimization problem in (77) by using standard constrained optimization schemes. However an alternative technique which circumvents the usage of constrained methods is proposed.

The technique involves iterative solutions of the generalized unconstrained closest boundary injection. The convergence is very fast and the required solution is found in only a few iterations as will be illustrated in the example. For a given infectiui, $\underline{\Sigma}_{g}$, one san find i.ts closest supporting hyperplane or feasibility plane $\underline{\alpha}_{0}^{T} z=0$. Suppose an injection $\underline{y}_{0}$ is defined such that it lies on the plane $\alpha_{0} \mathrm{~T}_{0}=0$ and the affine manifold $\Omega$ representing all the constraints imposed on the boundary injection as shown in Figs. 4.12 and 4.13. Similarly there exists a supporting hyperplane $\underline{\alpha}_{1}{ }^{T}=0$ that is closest to $\underline{y}_{0}$ and another injection $\underline{y}_{1}$ can be found such that it belongs to the intersection. of the plane $\underline{\alpha}_{1}^{T} \underline{z}=0$ and the affine manifold $\Omega$. In general we can define 


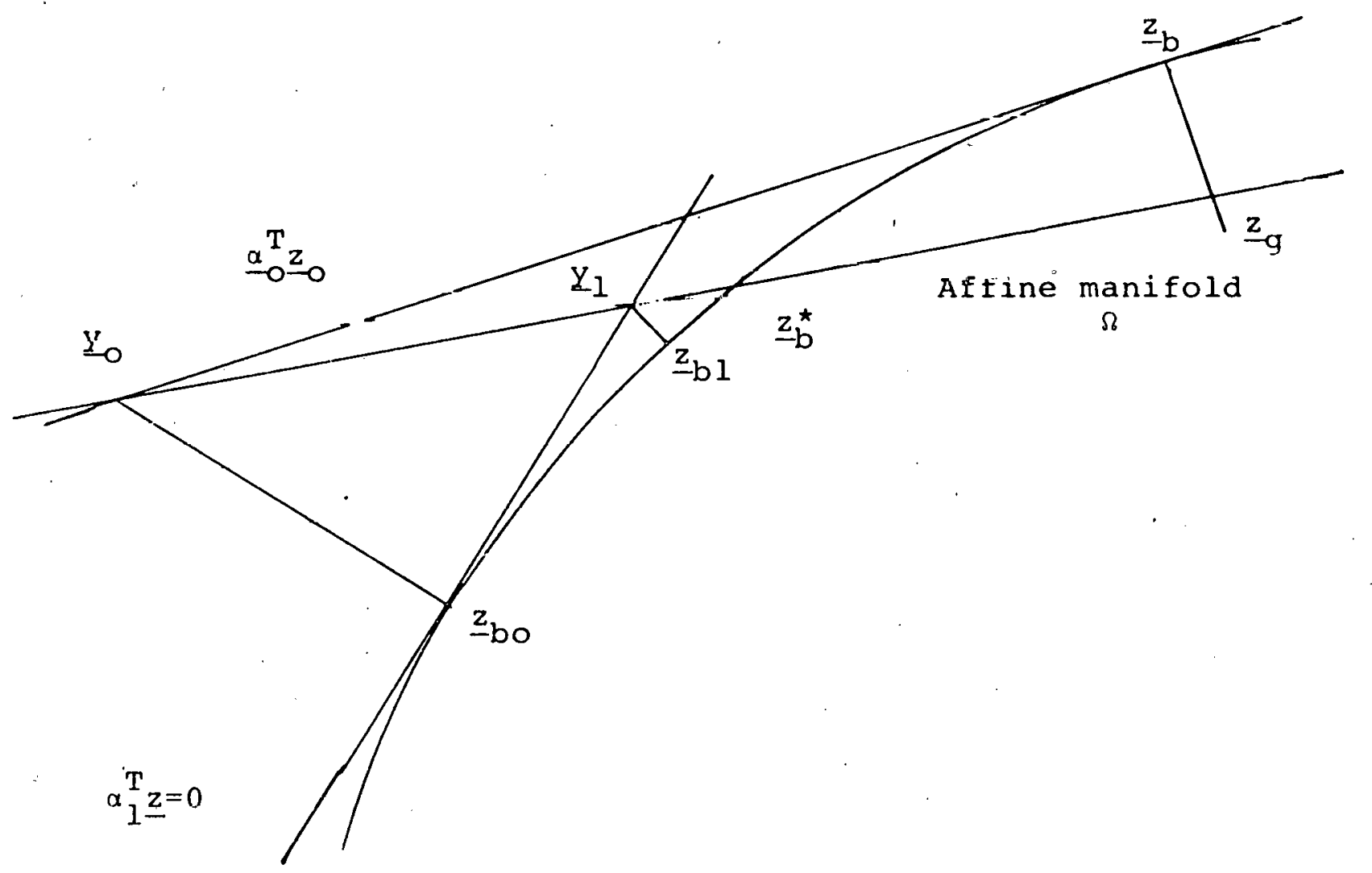

FIGURE 4.12. Geometrical illustration of the constrained : closest boundary injection $-\underline{z}_{b}^{\star}$. 


$$
\underline{y}_{i} \varepsilon\left\{\left[\underline{\alpha}_{i}^{\mathrm{T}} \underline{z}=0\right] \cap \Omega\right\} \quad i=1, \ldots, L
$$

and has the form

$$
\underline{y}_{i}=\left[\underline{u}_{i}, \underline{p}\right]
$$

where $\underline{p}$ is a fixed vector parameter and $\underline{u}_{i}$ corresponds to the free variable. In its simplest form $\underline{u}_{i}$ has one element. The number of free variables or the dimension of $\underline{u}_{i}$ depends on the number of planes $\underline{B}_{i}^{T} \underline{z}=k_{i}$ defining the constraints, $\Omega$. The number of free variables is equal to the dimension of the intersection of $R_{z}$ and the affine manifold $\Omega[3,12,15]$.

More often than not, the dimension of the vector will be greater than 1 . In this case $\underline{u}_{i}$ is chosen such that the Euclidean norm $\left\|\underline{u}_{i}-\underline{u}_{g}\right\|$ is minimum where $\underline{z}_{g}=\left[\underline{u}_{g}, \underline{p}\right]$. Geometrically this is illustrated in Fig. 4.13. It is not dif-. ficult to see that since

$$
\underline{\alpha}_{i}^{T} \underline{y}_{i}=0
$$

which implies

$$
\left[\underline{\alpha}_{\mathrm{ui}}^{\mathrm{T}}, \underline{\alpha}_{\mathrm{pi}}^{\mathrm{T}}\right]\left[\begin{array}{l}
\underline{u}_{i} \\
\underline{p}
\end{array}\right]=0
$$

that is,

$$
\stackrel{\alpha}{u i}^{\mathrm{T}} \underline{u}_{i}=-\underline{\alpha}_{-p}^{\mathrm{T}} \underline{\underline{ }} \triangleq \mathrm{k}_{\mathrm{u}}
$$

since 


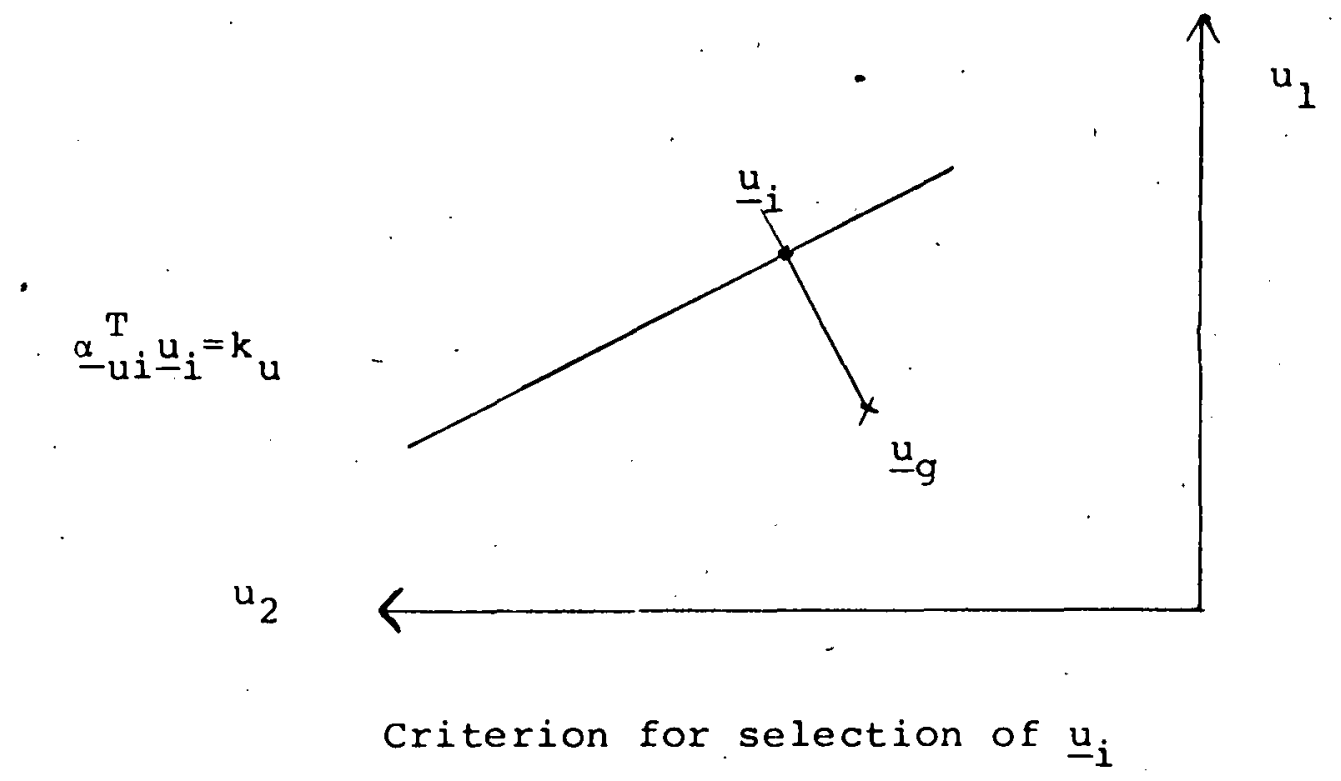

(a)

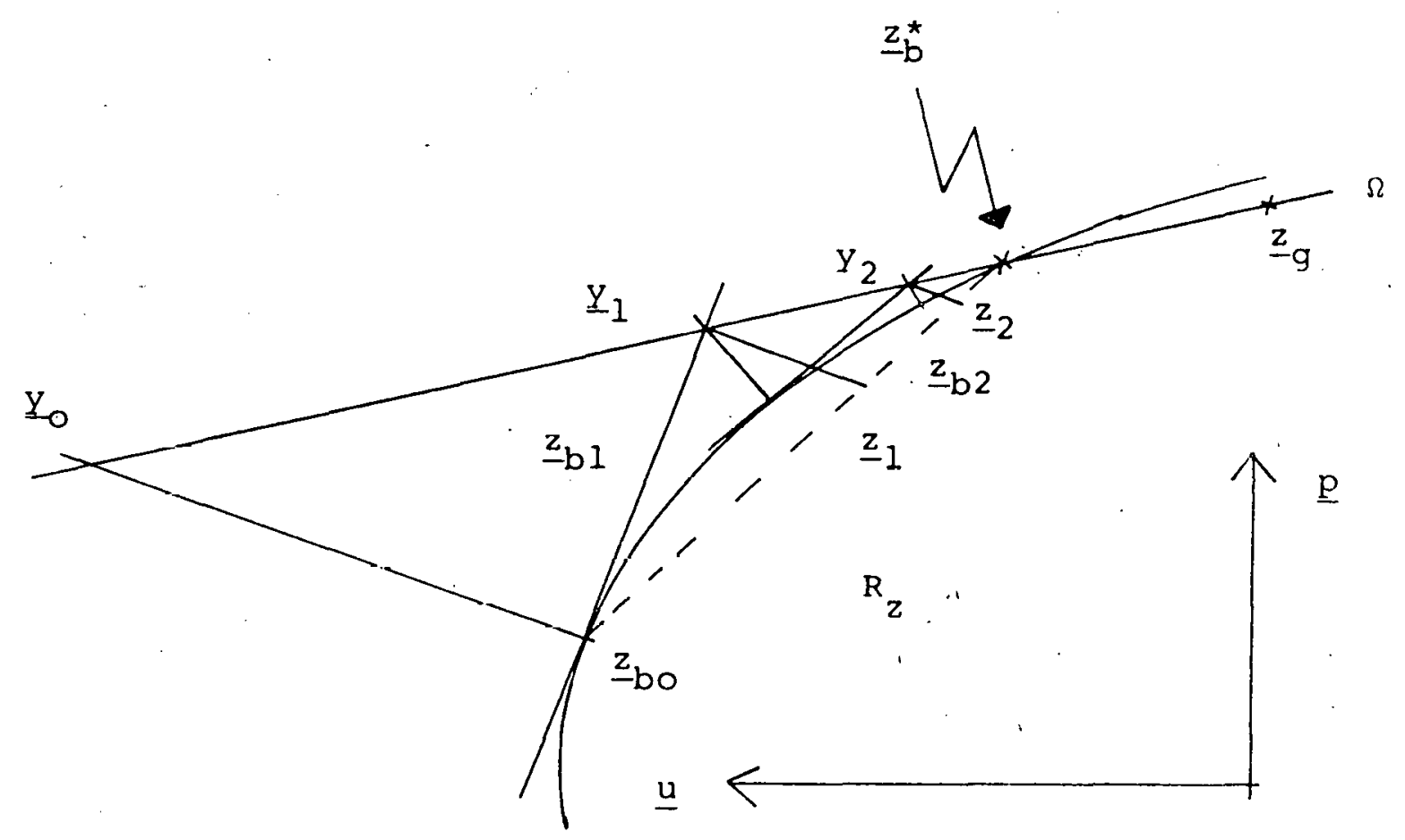

(b)

FIGURE 4.13. Convergence of $\underline{y}_{i}$ and $z_{p j}$ to the constrained closest boundary injection $\underline{z}^{*} \mathrm{~b}$. 


$$
\underline{\alpha}_{i}^{T}=\left[\underline{\alpha}_{u i}^{T}, \underline{\alpha}_{p i}^{T}\right] \text { and } \quad \underline{y}_{i}^{T}=\left[\underline{u}_{i}^{T}, \underline{p}^{T}\right]
$$

Then for the minimum of $\left\|\underline{u}_{i}-\underline{u}_{g}\right\|, \underline{u}_{i}$ is chosen such that.

$$
\underline{u}_{i}=\underline{u}_{g}+\sigma \underline{\alpha} i
$$

where

$$
\sigma=\frac{\mathrm{k}_{\mathrm{u}}-\underline{\alpha}^{\mathrm{T}} \underline{u}_{\mathrm{u}}}{\underline{\alpha}_{\mathrm{u} i}^{\mathrm{T}} \underline{\alpha}_{u i}}
$$

The convergence of this scheme has proven very reliable and rapid in all the tests made in systems of up to nine dimensions., A rigorous proof of convergence is being developed.

\section{Examples}
A 5-bus system
from Stagg and El-

Abiad [14] is used to illustrate the concept of tle congtrainod closest boundary injection.

An operating injection of the form

$$
\underline{\mathrm{z}}_{\mathrm{g}}=(-0.6,-0.1,-0.4,-0.05,-0.45,-0.15,0.48,1.0,1.0)
$$

where

$$
\underline{z}=\left(P_{1}, Q_{1}, P_{2}, Q_{2}, P_{3}, Q_{3}, P_{4}, V_{4}^{2}, V_{5}^{2}\right)
$$

is taken for illustrative purposes. Its load flow solution is summarized in Table 4.10 . 
TABLE 4.10

Load Flow Solutions of $\underline{z}_{g}$

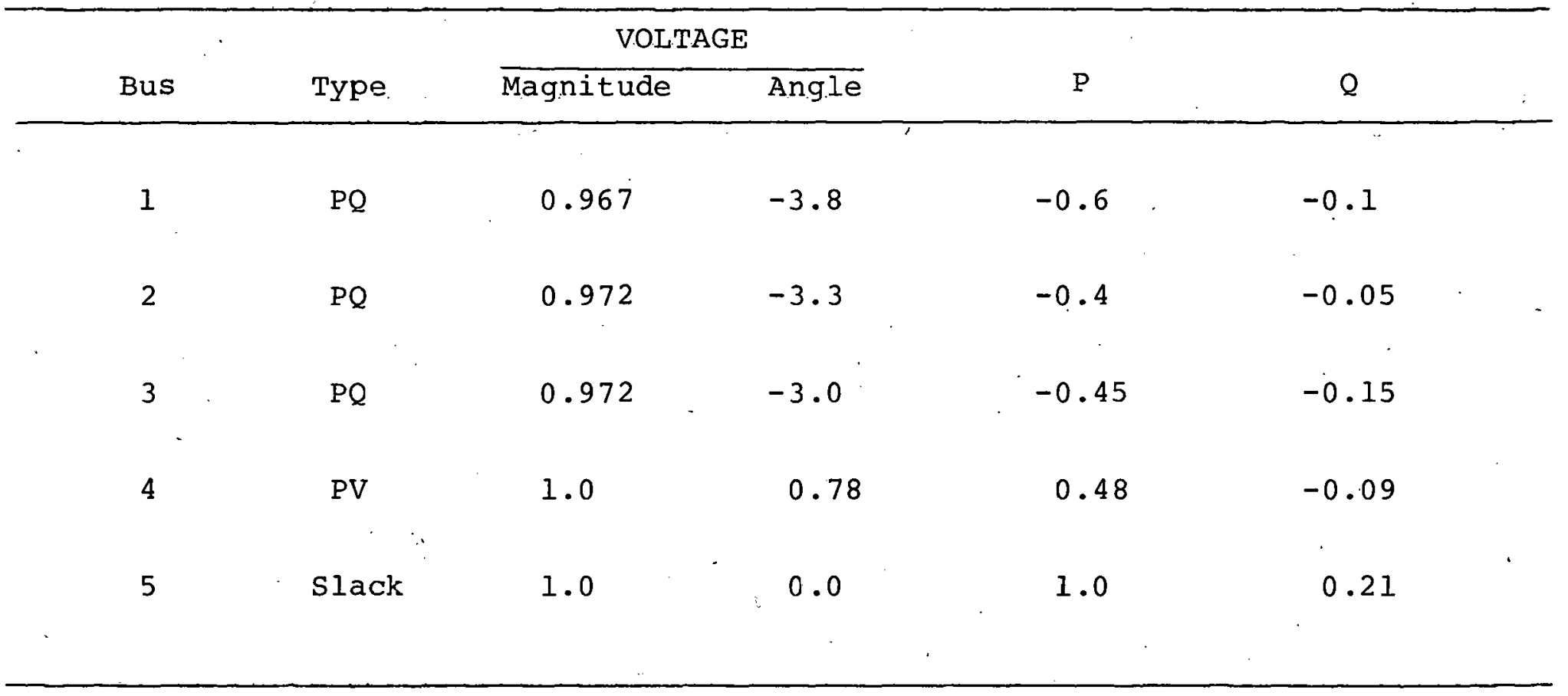


Suppose that at each of the buses we require the following constraints to hold,

At bus 1,

$$
\mathrm{Q}_{1}=1 / 6 \mathrm{P}_{1}
$$

At bus 2,

$$
\mathrm{Q}_{2}=1 / 8 \mathrm{P}_{2}
$$

At bus 3,

$$
\mathrm{Q}_{3}=1 / 3 \mathrm{P}_{3}
$$

At bus 4 ,

$$
\mathrm{v}_{4}^{2}=1.0
$$

At bus 5,

$$
\mathrm{v}_{5}^{2}=1.0
$$

Since by definition we have trom (81)

$$
y_{i} \varepsilon\left\{\left(\underline{\alpha}_{i} \underline{z}_{i}=0\right) \cap \Omega\right\}
$$

Then, $y_{i}$ must lie on the hyperplanes defined by $(88),(89),(90)$ (91), and (92) which implies for each $\underline{y}_{i}$ and $\underline{\alpha}_{i}$

$$
\underbrace{\left[\mathrm{P}_{1}, 1 / 6 \mathrm{P}_{1}, \mathrm{P}_{2}, 1 / 8 \mathrm{P}_{2}, \mathrm{P}_{3}, 1 / 3 \mathrm{P}_{3}, \mathrm{P}_{4}, 1.0,1.0\right]{ }_{i}^{\mathrm{T}} \underline{\alpha}_{i}=0}_{\underline{y}_{i}}
$$

that is, 


$$
\begin{aligned}
& P_{1}\left(\alpha_{1}+\alpha_{2} / 6\right)+P_{2}\left(\alpha_{3}+\alpha_{4} / 8\right)+P_{3}\left(\alpha_{5}+\alpha_{6} / 3\right)+P_{4} \alpha_{7} \\
& =-\left(\alpha_{8}+\alpha_{9}\right)
\end{aligned}
$$

where

$$
\underline{\alpha}_{i}=\left(\alpha_{1}, \alpha_{2}, \alpha_{3}, \alpha_{4}, \alpha_{5}, \alpha_{6}, \alpha_{7}, \alpha_{8}, \alpha_{9}\right)^{\mathrm{T}}
$$

Equation (93) is of the form

$$
\stackrel{\alpha}{-}_{u i}^{\mathrm{T}} \underline{u}_{i}=k_{u i}
$$

where

and

$$
\begin{aligned}
\underline{\alpha}_{u i} & =\left[\left(\alpha_{1}+\alpha_{2} / 6\right),\left(\alpha_{3}+\alpha_{4} / 8\right),\left(\alpha_{5}+\alpha_{6} / 3\right), \alpha_{7}\right]^{\mathrm{T}} \\
\mathrm{k}_{\mathrm{ui}} & =-\left(\alpha_{8}+\alpha_{9}\right) \\
\underline{u}_{\mathrm{i}} & =\left[\mathrm{P}_{1}, \mathrm{P}_{2}, \mathrm{P}_{3}, \mathrm{P}_{4}\right]^{\mathrm{T}}
\end{aligned}
$$

But the injection $\underline{y}_{i}$ has the form

$$
\underline{y}_{i}=\left[\underline{u}_{i}^{T}, \underline{p}^{T}\right]^{T}
$$

and $u_{i}$ is chosen such that $\left\|\underline{u}_{i}-\underline{u}_{q}\right\|$ is minimum which implies

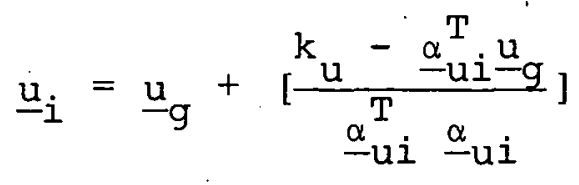

where

$$
\underline{u}_{g}=[-0.6,-0.4,-0.45,0.48]^{\mathrm{T}}
$$

A base case study is run using the unconstrained closest boundary program and $\underline{z}_{g^{*}}$ From the closest hyperplane 
TABLE 4.11

Summary of Results of Constrained Closest Boundary Condition

$$
\underline{z}_{g}=(-0.6,-0.1,-0.4,-0.05,-0.45,-0.15,0,48,1.0,1.0)
$$

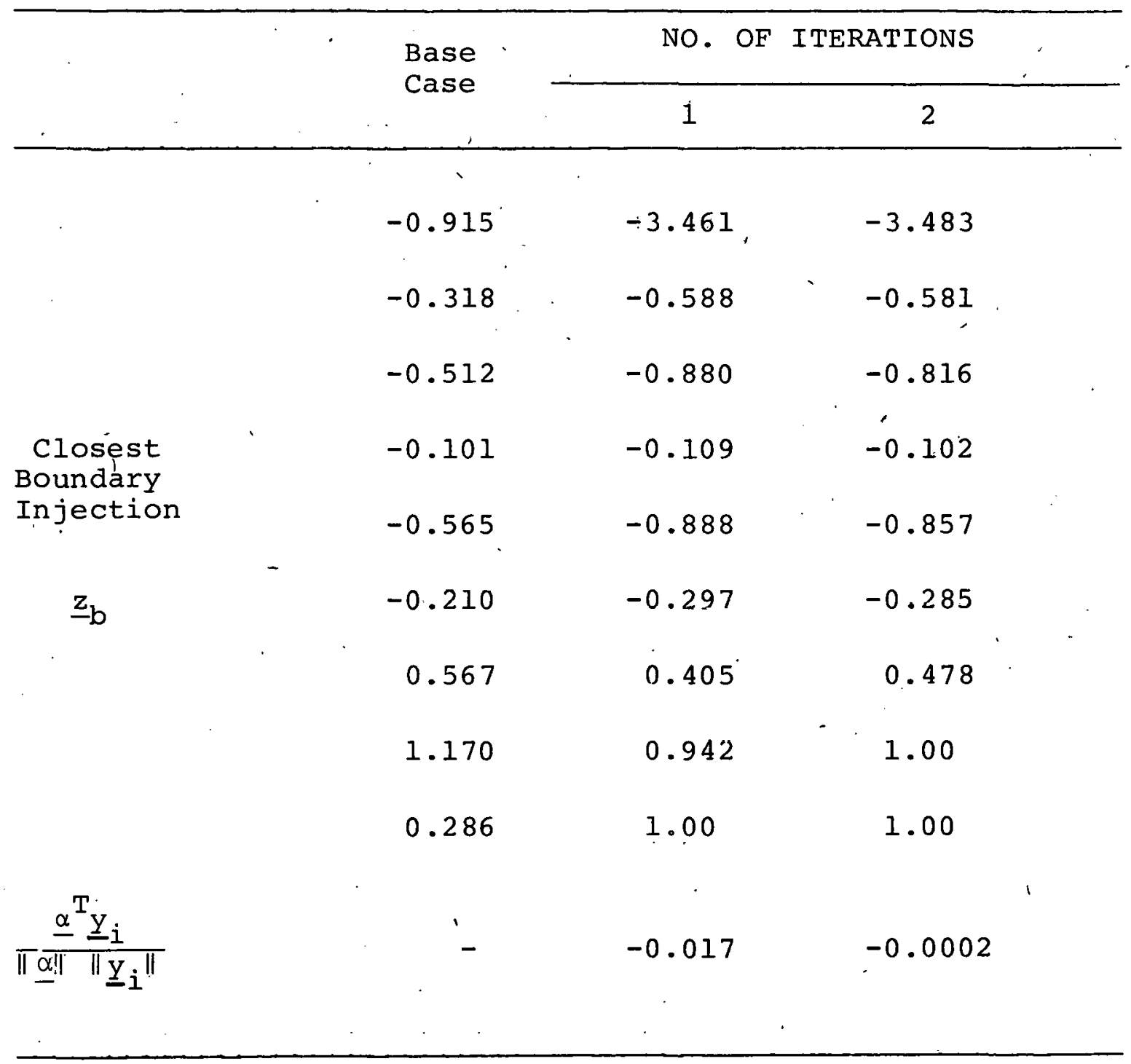


$\underline{\alpha}_{0}^{\mathrm{T}} \underline{z}=0$ found, one computes $\mathrm{y}_{1}$ from Equations (95) and (96). This procedure is similarly repeated until convergence is achieved.

Table 4.11 summarizes the result of the search for the constrained closest boundary injection to the given injection $\underline{z}_{g}$. It has the form,

$$
\begin{aligned}
\underline{z}_{b}^{*}= & {[-3.483,-0.581,-0.816,-0.102,-0.857,-0.285,0.48,} \\
& 1,1]
\end{aligned}
$$

which satisfies the constraints in (88), (89), (90), (91), and (92). 
5.0 CHARACTERIZATION OF THE SET OF SECURE SOLUTIONS IN THE VOLTAGE SPACE

\subsection{Introduction}

The characterization of the set of secure solutions to a power system under certain load distribution and engineering limitations, is generally recognized as an important part of the overall system security. In the past this problem had been usually studied under the heading of either " optimum load flow " or simply " constrained load flow" with much limited scope. The ultimate goal was usually limited to finding one single solution - called the optimum or secure solution - to a set of non-linear algebraic equations

$$
\underline{Z}_{L}=\underline{Z}(\underline{X}) \cdot \underline{0}
$$

satisfying also a set of constraints such as

$$
\begin{aligned}
& \underline{U}^{\mathrm{m}} \leqslant \underline{U}=\underline{U}(\underline{X}) \leqslant \underline{U}^{M} \\
& \underline{Y}^{\mathrm{m}} \leqslant \underline{Y}=\underline{Y}(\underline{X}) \leqslant \underline{Y}^{M}
\end{aligned}
$$

while extremizing a certain objective function.

$\mathrm{Z}_{\mathrm{L}}$ in (1), the load flow equations, represents the $2 \mathrm{~m}$ dimensional vector of the specified real and reactive loads. The set of inequalities in (2) denote the limitations on the 
controllable injections. The vector $\underline{U}=\underline{U}(\underline{X})$. here contains ali the $2 n+1$ variables at the slack and $P V$ buses and $\underline{U}^{\mathbb{M}}$ and $\underline{U}^{\mathrm{m}}$ are respectively the vector of upper and lower bounds on these variables. Variables such as voltage magnitudes at $P Q$ buses or line power flows which are not directly controllable but must remain within specified limits to guarantee satisfactory operation, are entries of the vector $\underline{Y}=\underline{Y}(\underline{X})$ in (3). The vectors $\underline{Y}^{M}$ and $\underline{Y}^{m}$ contain their respective upper and lower bounds.

The problem in tho context of security requires. a broader treatment. Here one is primarily concerned with the existence of such solutions. If a solution exist, then the next step is to characterize the set of all secure solutions instead of a secure solution. Finally, one may examine members of the set to find the most secure and/or economical solution against a certain security and/or economic measure.

In regard to the on-line control of the power systems, nonexistence of a solution oatisfying (1) through (3) can lead to costly and useless numerical runs, while suspecting a slow convergent solution. In such occasions, the resulted delay in taking the proper action by dispatcher can very well lead to violation of some operating specifications, e.g. abnormal voltage levels at load buses or excessive thermal heating of a transformer, or it may result in a more serious situation which would require the immediate outage of the overloaded 
equipments.

Using the set theoretic approach, the problem can be defined in a more clear and unified manner. Consider first the following definitions:

$$
S_{X}=\left\{\underline{X} ; \underline{U}^{m} \leqslant \underline{U}(\underline{X}) \leqslant \underline{U}^{M i} ; \underline{Y}^{m} \leqslant \underline{Y}(\underline{X}) \leqslant \underline{Y}^{M}\right\}
$$

Thus $S_{X}$ is the set of all $\underline{X}$. satisfying all inequality constraints. Next define the set

$$
L_{X}=\left\{\underline{X} ; \underline{X} \varepsilon \underline{Z}(\underline{X})=\underline{Z}_{L}\right\}
$$

which is the set of all $\underline{x}$ belonging to the $n+1$ dimensional manifold of the intersection of the $2 \mathrm{~m}$ equality constraints $\underline{Z}(\underline{X})=\underline{Z}_{I}$. The set of all secure solutions, $\underline{S}_{X}\left(\underline{Z}_{L}\right)$, is then

$$
s_{X}\left(\underline{Z}_{I}\right)=s_{X} \cap I_{X}
$$

Now interms of $S_{X}\left(\underline{Z}_{L}\right)$ the question of existence of a secure solution is simply determining whether $S_{X}\left(\underline{Z}_{L}\right)$ is empty or not. The characterization of all secure solutions can be studied through examining the elements of $\mathrm{S}_{X}\left(\underline{z}_{L}\right)$ or in other. words through determining its shape, its size, its explicit description and its dependence on the various system parameters. Unfortunately the high non-linearity of $S_{X}\left(\underline{Z}_{L}\right)$ accompanied with the large dimensionality of the problem renders its implementation for on-line control very difficult. 
In this section, we examine one particular approach for analyzing the characteristics of $S_{X}\left(Z_{L}\right)$. The basic idea is to surround $S_{X}\left(\underline{Z}_{\bar{L}}\right)$ by a set, $\hat{S}_{X}\left(\underline{Z}_{L}\right)$, containing the former set, and described by a number of explicit linear inequalities in $\underline{x}$. Thus

$$
\hat{S}_{X}\left(\underline{z}_{L}\right) \supseteq S_{X}\left(\underline{z}_{L}\right)
$$

$\hat{S}_{X}\left(Z_{L}\right)$ is in turn the intersection of $\hat{S}_{X}$ and $\hat{\mathrm{L}}_{X}$, i.e.

$$
\hat{S}_{X}\left(\underline{Z}_{L}\right)=\hat{S}_{X} \cap \hat{L}_{X}
$$

where $\hat{S}_{X}$ and $\hat{L}_{X}$ are the linear counter-parts of $S_{X}$ and $\mathrm{I}_{X}$ respectively.

The fact that the linear inequalities which form $\hat{S}_{X}\left(\underline{Z}_{L}\right)$ surround $S_{X}\left(Z_{L}\right)$ very tightly and hence make $\hat{S}_{X}\left(Z_{L}\right)$ a " close " approximation to $S_{X}\left(\underline{Z}_{L}\right)$, permits us to conclude certain characteristics of $S_{X}\left(\underline{Z}_{L}\right)$ with much ease.

The key to the approach is employing the load flow equations in rectangular coordinates in their current form. i.e.

$$
\begin{aligned}
& \operatorname{Re}\left(I_{j}\right)=\left(\frac{P_{i}}{v_{i}^{2}}\right) e_{i}+\left(\frac{Q_{i}}{v_{i}^{2}}\right) f_{i}=a_{i}(\underline{X}) \\
& \operatorname{Im}\left(I_{i}\right)=\left(\frac{P_{i}}{v_{i}^{2}}\right) f_{i}-\left(\frac{Q_{i}}{V_{i}^{2}}\right) e_{i}=b_{i}(\underline{X})
\end{aligned}
$$

where $I_{i}$ is the complex current injections into bus $i$ and 


$$
\begin{aligned}
& a_{i}(\underline{X})=\sum_{K=1}^{N}\left(G_{i k} e_{k}-B_{i k} f_{k}\right) \\
& b_{i}(\underline{X})=\sum_{K=1}^{N}\left(G_{i k} f_{k}+B_{i k} e_{k}\right)
\end{aligned}
$$

The quasi-linearity of equations (5) and (6) in $\underline{X}$ permits us to derive the linear ineqalities which form $\hat{S}_{X}\left(\underline{Z}_{L}\right)$.

\subsection{Security Constraints}

The set of constraints imposed on $\underline{U}$ and $\underline{Y}$ in (2) and (3) are the security constraints which are to be respected at all times. As mentioned earlier, these are essentially engineering limitations, compromises between conflicting design objectives. In this section we briefly examine their nature and relative importance.

i) Genoration limits:

At all generation buses the power injections are bounded. The real injections are restricted from above by generators' generation capabilities and from below by the minimum opening-time of the valve control mechanism. Thus

$$
P_{i}^{m} \leqslant P_{i}=P_{i}(\underline{x}) \leqslant P_{i}^{M} \quad i=1, \ldots, n
$$

The reactive power injections are bounded mainly to avoid generator stability and loss of synchronism. 


$$
Q_{i}^{m} \leqslant Q_{i}=Q_{i}(X) \leqslant Q_{i}^{M} \quad . i=1, \ldots n
$$

Note that with setting $Q_{i}^{m}=0$ or $Q_{i}^{\mathbb{M}}=0$, one can have the generator $i$ on over-excited or under-excited operating mode respectively.

ii) Voltage magnitude limits:

The voltage limits are specially critical at the load buses and must be restricted to withina few per-cent. Such hard constraints would ensure the delivery of power to conoumers al lhe rated voltage. The remaining buses (i.e. PV and slack) on the other-hand are subject to much softer constraints since their bounds are set in regard to insulation and over-voltage limits of the components involved. The voltage limits are

$$
\left(v_{i}^{m}\right)^{2} \leqslant v_{i}^{2}=e_{i}^{2}+f_{i}^{2} \leqslant\left(v_{i}^{M}\right)^{2} \quad i=1, \ldots, N
$$

\section{iii) Thermal Iimits:}

Transmission lines are also eubject to limits on the amount of power which can be sent through them. This is the line thermal limit.8

$$
P_{1 k}^{m} \leqslant P_{I k}=P_{1 k}(\underline{x}) \leqslant P_{1 k}^{M I}
$$

where $P_{1 k}$ is the real power sent from bus 1 to bus $k$. Violation of the upper bound of thermal limits can. 
cause excessive heating of transmission lines which is followed by slacking down, loss of mechanical strength and finally rupture. The lower bounds can be set equal to zero once the direction of power flows under normal operation are known.

\section{$5 \cdot 3$ Auxiliary Inequalities}

The set of inequalities listed in the preceeding section are not appropriate for our purpose in their present form. We intend to derive a new set of inequalities which suits our formulation, using the original ones.

By restricting the branch flows and voltage, levels to be within certain limits, we are essentially limiting the voltage angles at all buses. Appendix A-5 describes in detail how one can establish relations such as

$$
\theta_{i}^{m} \leqslant \theta_{i} \leqslant \theta_{i}^{M} \quad i=2, \ldots, N
$$

using the original inequalities.

The second group of inequalities which are needed, are:

$$
\begin{array}{ll}
\lambda_{i}^{m} \leqslant \frac{P_{i}}{v_{i}^{2}} \leqslant \lambda_{i}^{M} & i=1, \ldots, n \\
\gamma_{i}^{m} \leqslant \frac{Q_{i}}{v_{i}^{2}} \leqslant \gamma_{i}^{M} &
\end{array}
$$


A full detail on how the above bounds can be set is given in appendix $\mathrm{B}-5$.

5.4 Formulation of $\hat{\mathrm{S}}_{\mathrm{X}}$

Consider a pair of inequalities stated in (11) and (13) corresponding to a single bus $i$. In the plane $e_{i}-f_{i}$, they represent a concave region shown shadded in fig.(5-1). By virtue of the fact that the limits on $\theta_{i}$ are usually very close, this region can be closely surrounded by 5 lines, forming a convex linear set, fig. $(5-2)$.

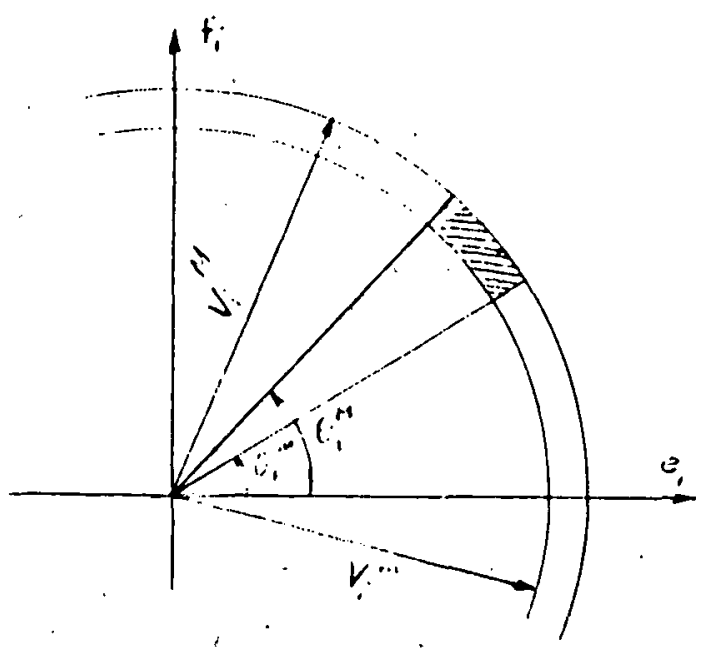

Fig. $(5-1)$

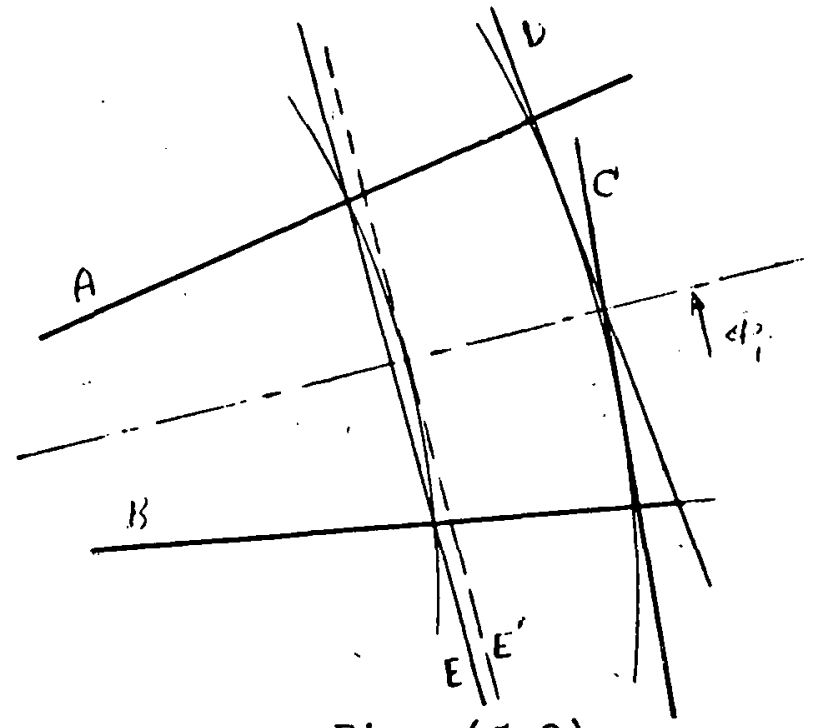

Fig. $(5-2)$

The convex region is defined by the following inequalities

Iine $A,(\sin \theta \underset{i}{M}) e_{i}-(\cos \theta \underset{i}{M}) f_{i} \geqslant 0$

Iine $B:-(\sin \theta \underset{i}{m}) e_{i}+(\cos \theta \underset{i}{m}) f_{i} \geqslant 0$

line $C, \quad\left(\cos \delta{ }_{i}^{m}\right) e_{i}+\left(\sin \delta m_{i}^{m}\right) f_{i} \leqslant v_{i}^{M}$ 
line $D:\left(\cos \delta i_{i}^{M}\right) e_{i}+\left(\sin \delta{ }_{i}^{M}\right) f_{i} \leqslant v_{i}^{M}$

line $E:\left(\cos \phi_{i}\right) e_{i}+\left(\sin \phi_{i}\right) f_{i} \geqslant v_{i}^{m}\left[m+(1-m) \cos \left(\phi_{i}-\theta i_{i}^{m}\right)\right] \quad(20)$ where

$$
\begin{aligned}
& \phi_{i}=\frac{1}{2}\left(\theta_{i}^{M}+\theta_{i}^{m}\right) \\
& \delta_{i}^{m}=\frac{1}{2}\left(\theta_{i}^{m}+\phi_{i}\right) \\
& \delta_{i}^{M}=\frac{1}{2}\left(\theta_{i}^{M}+\phi_{i}\right)
\end{aligned}
$$

Note that line $E$ can move toward line $E^{\prime}$ by changing the parameter $m$ from 1 to 0 .

Inequalities (16) to (20) are practically the linear expressions which replace inequalities (11) and (12), and thus they are part of the linear set $\hat{\mathrm{S}}_{X}$. To include the inequalities corresponding to power injections at PV buses, we are to examine linear combinations of $a_{i}(\underline{X})$ and $b_{i}(\underline{X})$ at these buses. From equations (4) and (5)

$$
\alpha a_{i}(\underline{X})+\beta b_{i}(\underline{X})=\frac{P_{i}}{v_{i}^{2}}\left(\alpha e_{i}+\beta f_{i}\right)+\frac{Q_{i}}{v_{i}^{2}}\left(\alpha f_{i}-\beta e_{i}\right)
$$

where and are any two real constants. The reason that we are interested in relations such as (22), rather than (4) and (5), is that we need to have a priori knowledge of the sign of the expressions which multiply $\frac{P_{i}}{v_{i}^{2}}$ and $\frac{Q_{i}}{v_{i}^{2}} \cdot$ In 
equations (4) and (5), though we know $e_{i}$, is always positive, there seems to be no simple way to justify any speculation on the sign of $f_{i}(i=2, \ldots, N)$. But on the contrary, existence of relations such as (16) and (17) clearly indicates that by proper choice of $\alpha$ and $\beta$ one can easily have, for example,

$$
\begin{aligned}
& \alpha e_{i}+\beta f_{i} \geqslant 0 \\
& \alpha f_{i}-\beta e_{i} \geqslant 0
\end{aligned}
$$

The motive for establishing relations such as (23) and (24) should be obvious by now. Multiplying (23) and (24) by (14) and (15) repectively and adding up the resulting inequalities and then using equation (22) leads us to

$\alpha a_{i}(\underline{X})+\beta b_{i}(\underline{X})\left[\begin{array}{l}\leqslant \lambda{ }_{i}^{M}\left(\alpha e_{i}+\beta f_{i}\right)+\gamma_{i}^{M}\left(\alpha f_{i}-\beta e_{i}\right) \\ \geqslant \lambda{ }_{i}^{m}\left(\alpha e_{i}+\beta f_{i}\right)+\gamma_{i}^{m}\left(\alpha f_{i}-\beta e_{i}\right)\end{array}\right.$

Inequalilies in (25) are linear in $\mathrm{X}$ and replace nonlinear inequalities such as (9) in $S_{X}$.

Comparing inequalities (16) and (17) with that of (23) and (24), one notes that there is no similiar relation between coefficients of $e_{i}$ and $f_{i}$ as exists in the latter one. However if we assume that

$$
\left|\theta_{i}^{M}-\theta_{i}^{m}\right| \leqslant 90^{\circ} \quad . i=1, \ldots, N
$$


i,e. the shadded area in fig. (51) always falls inside a $90^{\circ}$ sector, the boundary lines of this $90^{\circ}$ sector satisfy

$$
\begin{aligned}
& \left(\cos \phi_{i}+\sin \phi_{i}\right) e_{i}+\left(\sin \phi_{i}-\cos \phi_{i}\right) f_{i} \geqslant 0 \\
& \left(\cos \phi_{i}-\sin \phi_{i}\right) e_{i}+\left(\sin \phi_{i}+\cos \phi_{i}\right) f_{i} \geqslant 0
\end{aligned}
$$

where $\phi_{i}$ is chosen as the bisector angle of the $90^{\circ}$ sector - see fig. (2). The obvious choice of ' $\alpha$ and $\beta$ is then:

$$
\begin{aligned}
& \alpha_{i}=\cos \phi_{i}+\sin \phi_{i} \\
& \beta_{i}=\sin \phi_{i}-\cos \phi_{i}
\end{aligned} \quad \quad i=1, \ldots, n
$$

$\alpha$ and $\beta$ in (28) and (29) are subscripted to indicate that at each bus $i$ a different pair of $\alpha$ and $\beta$ has to be computed.

Now suppose instead of starting with inequalities (23) and (24), one starts with

$$
\begin{aligned}
& \alpha^{\prime} e_{i}+\beta_{f}^{\prime} f_{i} \geqslant 0 \\
& \alpha^{\prime} f_{i}-\beta_{e_{i}}^{\prime} \leqslant 0
\end{aligned}
$$

Following the same steps, it follows

$$
\alpha^{\prime} a_{i}(\underline{x})+\beta^{\prime} b_{i}(\underline{x})\left[\begin{array}{l}
\lambda_{i}^{M}\left(\alpha^{\prime} e_{i}+\beta^{\prime} f_{i}\right)+\gamma_{i}^{m}\left(\alpha^{\prime} f_{i}-\beta^{\prime} e_{i}\right) \\
\geqslant \lambda_{i}^{m}\left(\alpha^{\prime} e_{i}+\beta^{\prime} f_{i}\right)+\gamma_{i}^{M}\left(\alpha^{\prime} f_{i}-\beta^{\prime} e_{i}\right)
\end{array} .\right.
$$


To gèt $\alpha^{\prime}$ and $\beta^{\prime}$; we need to multiply (26) by -1 , while (27) is kept intact. The result of comparison with (30) and (31) is then

$$
\begin{aligned}
& \alpha_{i}^{\prime}=\cos \phi_{i}-\sin \phi_{i}=-\beta_{i} \\
& \beta_{i}^{\prime}=\cos \phi_{i}+\sin \phi_{i}=\alpha_{i}
\end{aligned}
$$

Inequalities (32) and (33) complete the set of Iinear inequalities which form $\hat{S}_{X}$.

5.5 Formulation nf $\hat{\mathrm{I}}_{\mathrm{x}}$

At the $m P Q$ buses, equation (22) can be re-written as

$$
v_{i}^{2}\left[\alpha a_{i}(\underline{x})+\beta b_{i}(\underline{x})\right]=\left(\alpha P_{i}^{1}-\beta Q_{i}^{l}\right) e_{i}+\left(\alpha Q_{i}^{1}+\beta P_{i}^{l}\right) f_{i}
$$

where $P_{i}$ and $Q_{i}$ are set equal to $P_{i}^{l}$ and $Q_{i}^{l}$, their specified values. If one chooses the multiplicand of $e_{i}$ and $f_{i}$ to be $\mathrm{k}_{1}$ and $\mathrm{k}_{2}$, namely

$$
\begin{aligned}
& \alpha P_{i}^{I}-\beta Q_{i}^{I}=k_{1} \\
& \alpha Q_{i}^{I}+\beta Q_{i}^{I}=k_{2}
\end{aligned}
$$

such that the constants $k_{1}$ ard $k_{2}$ satisfy

$$
k_{1} e_{i}+k_{2} f_{i} \geqslant 0
$$

then by comparing (36) with (39), it follows 


$$
\alpha a_{i}(\underline{x})+\beta b_{i}(\underline{x}) \geqslant 0
$$

The constant $\alpha$ and $\beta$ in (40) are solutions to (37) and (38). The above inequality can be used to derive a pair of inequalities belonging to $\hat{\mathrm{L}}_{\mathrm{x}}$.

Multiplying (11) by (40) and substituting from (36), (37), and (38) in the resulting expression, one ends up with

$$
\alpha a_{i}(\underline{x})+\beta b_{i}(\underline{x}) \mid \begin{aligned}
& \leqslant \frac{1}{\left(v_{i}^{m}\right)^{2}}\left(k_{1} e_{i}+k_{2} f_{i}\right) \\
& \geqslant \frac{1}{\left(v_{i}^{M}\right)^{2}}\left(k_{1} e_{i}+k_{2} f_{i}\right)
\end{aligned}
$$

These two inequalities define a very narrow. wedge in the space of voltages as the possible location of the solutions. A typical cross-section of this wedge in the $e_{i}-f_{i}$ plane is shown in fig. (5-3). Tu reotrict further the location of the possible solutions, one can pick up another pair of constants satisfying ( 39 ), say $k_{3}$ and $k_{4}$, and by repeating the same steps obtain

$$
\alpha a_{i}(\underline{x})+\beta b_{i}(\underline{x}) \mid \begin{aligned}
& \leqslant \frac{1}{\left(v_{i}^{m}\right)^{2}}\left(k_{3} e_{i}+k_{4} f_{i}\right) \\
& \geqslant \frac{1}{\left(v_{i}^{M}\right)^{2}}\left(k_{3} e_{i}+k_{4} f_{i}\right)
\end{aligned}
$$

where $\alpha$ and $B$ in (42) are different from those in (41). The inequalities in (42) also define a very narrow wedge in the 


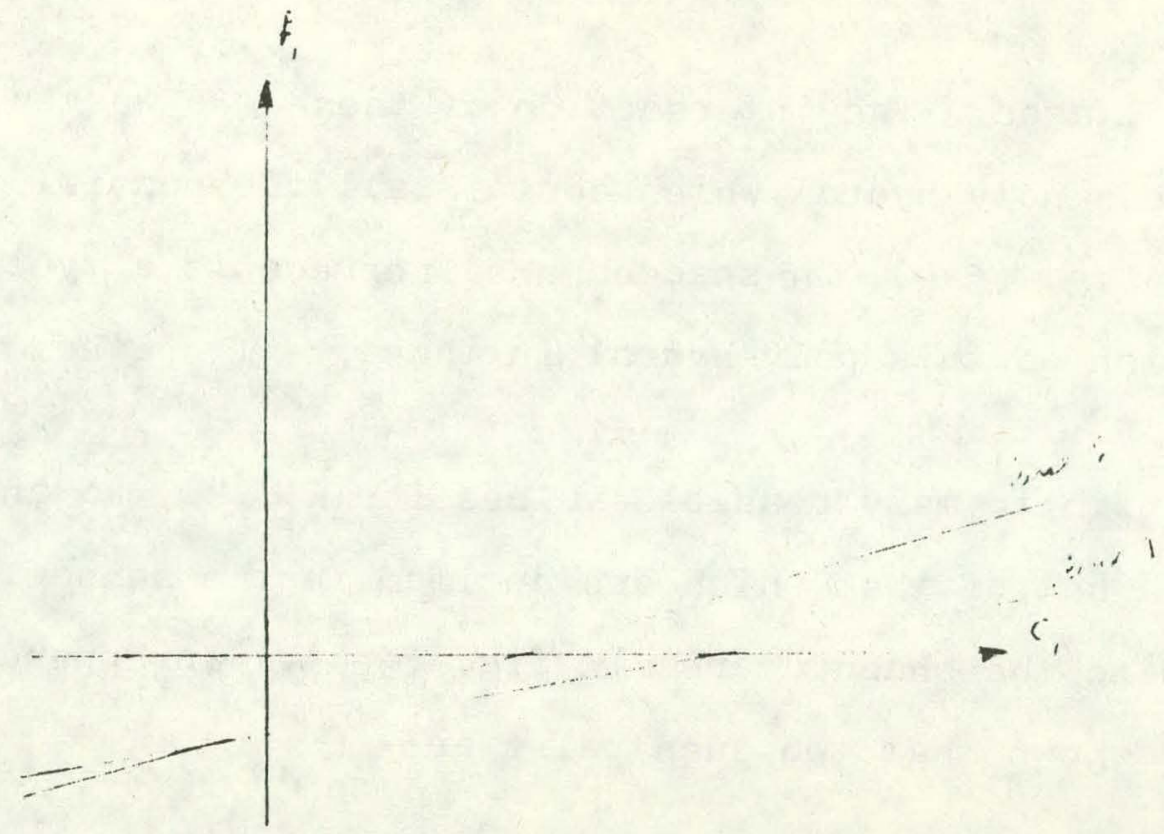

Fig. (5-3)

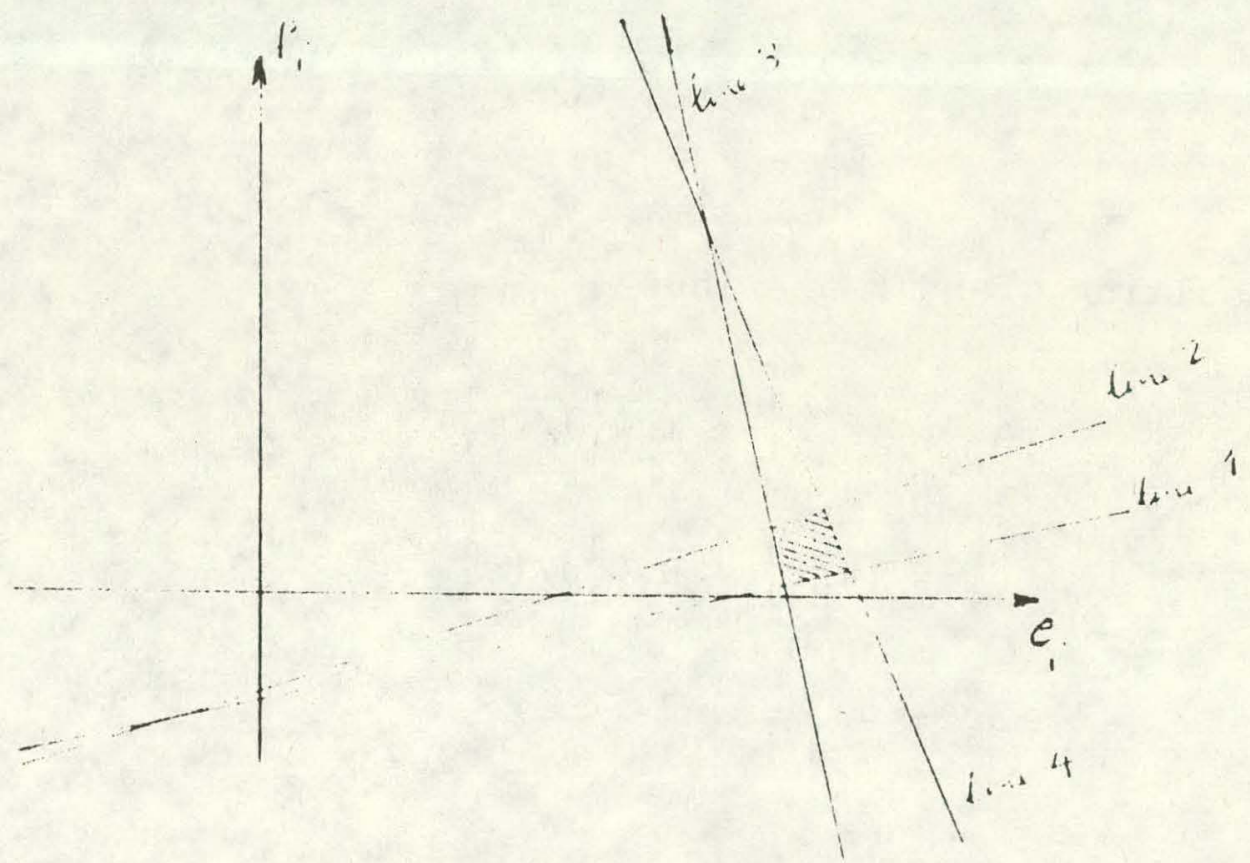

Fig. $(5-4)$ 
space of voltages. The intersection of these two wederes defines a bottom-less poly-hydral which contain all the possible solutions. In $f i_{\varepsilon,} \cdot(5-4)$, the shadded area represents a typical cross-section of this poly-hydral in the $e_{i}-f_{i}$ plane.

Of infinite many possible values for $k_{1}, k_{2}, k_{3}$ and $k_{4}$, there is a set of them which are optimum in the sense that they minimize the shadded area in fig. (5-4). In Appendix $\mathrm{C}-5$, it is shown that two such pairs are

$$
\begin{aligned}
& k_{1}=\cos \phi_{i}+\sin \phi_{i} \\
& k_{2}=\sin \phi_{i}-\cos \phi_{i} \\
& k_{3}=-k_{2} \\
& k_{4}=k_{1}
\end{aligned}
$$

The resulting $\alpha$ and $\beta$ are thus

$$
\begin{aligned}
& \alpha_{i}^{\prime \prime}=\left(k_{1} P_{i}^{l}+k_{2} Q_{i}^{l}\right) /\left(s_{i}^{l}\right)^{2} \\
& \beta_{i}^{\prime \prime}=\left(k_{2} P_{i}^{l}-k_{1} Q_{i}^{l}\right) /\left(s_{i}^{l}\right)^{2} \\
& \alpha_{i}^{\prime \prime \prime}=-\beta_{i}^{\prime \prime} \\
& \beta_{i}^{\prime \prime}=\alpha_{i}^{\prime \prime}
\end{aligned}
$$

where

$$
\left(S_{i}^{1}\right)^{2}=\left(P_{i}^{1}\right)^{2}+\left(Q_{i}^{1}\right)^{2}
$$


and super-scripts and sub-scripts are used to distinguish different sets of $\alpha$ and $\beta$ at a single bus and at different buses respectively. Note that " $i$ " here counts the $P Q$ buses.

Inequalities (41) and (42) are linear in $x$ and load dependent. The collection of all such inequalities form $\hat{L}_{x}$.

\subsection{Numerical Considerations}

At the slack bus, since one is able to set freely the imaginary parl of the voltage equal to zero, a'great deal of simplification in deriving the linear inequalities results. The relations (5), (6); and (11) reduce simply to

$$
\begin{aligned}
& \frac{P_{1}}{e_{1}}=a_{1}(\underline{x}) \\
& \frac{Q_{1}}{e_{1}}=-b_{1}(\underline{x})
\end{aligned}
$$

and

$$
v_{1}^{m} \leqslant e_{1} \leqslant v_{1}^{M}
$$

After evaluating $\gamma_{1}^{M}$ and $\gamma_{1}^{m}$ for $\left(\frac{P_{1}}{e_{1}}\right)$ and also $\lambda_{1}^{M}$ and $\lambda{ }_{1}^{m}$ for $\left(\frac{Q_{1}}{e_{1}}\right)$, according to Appendix $B-5$, one obtains

$$
\begin{aligned}
& \gamma_{1}^{m} \leqslant a_{1}(\underline{x}) \leqslant \gamma_{1}^{M} \\
& \lambda_{1}^{m} \leqslant-b_{1}(\underline{x}) \leqslant \lambda_{1}^{M}
\end{aligned}
$$

These are much simpler, than their counter-parts in (25) and (32). Note that equation (45) is linear in $\underline{x}$. 
At those $P Q$ buses where there is zero injections, i.e. $\mathrm{P}_{i}^{l}=Q_{\dot{i}}^{I}=0$, the corresponding inequalities are replaced simply by two linear equations, namely

$$
\begin{aligned}
& a_{i}(\underline{x})=0 \\
& b_{i}(\underline{x})=0
\end{aligned}
$$

The total number of inequalities which form $\hat{S}_{x}\left(\underline{z}_{L}\right)$ that is the collection of al-l inequalities forming $\hat{\mathrm{S}}_{\mathrm{x}}$ and $\hat{\mathrm{I}}_{\mathrm{x}}$ - is quite large. This would reduce efficiency and could cause storage problem when for characterizing $\hat{S}_{X}\left(\underline{z}_{L}\right)$, a linear programming (LP) approach is employed. A good deal of these inequalities are, however, redundant and by filtering them out the dimension of the problem can be reduced.

- The inequalities which form $\hat{\mathrm{I}}_{\mathbf{x}}$ are load-dependent and they rotate in the voltage space as load varies. This means a constraint which is inactive for certain load distributions could be active for some other load distributions. The inequalities forming $\hat{\mathrm{S}}_{\mathrm{x}}$, on the other hand, are load-independent and for a given network, with specific engineering limitations on its components, remain fixed. This allows one to filter out all the redundant constraints in the $\hat{S}_{x}$, using well known algorithms $[33,34,35]$. Our experience has shown that about $40 \%$ of the constraints for a small size network (8-bus system) turn out to be redundant. For larger networks it is expected that this per-centage rises sharply.' 
Since the imaginary part of the bus voltages, particularly at the $P Q$ buses, can accept positive or negative values, and in LP it is essential to have all the variables positive, one has to replace them by the difference of two positive variables. This would practically enlarge the LP tableau by $50 \%$. Noting that to have an initial feasible solution a superficial variable has to be also added to each and every constraint, the enormous dimensions of the tableau, for a large power system, can be visualized.

Both of the abuve prublems can be avolaed to a large extent by converting our primal LP problem to its dual, once an objective function is defined. In particular, the fact that the number of original variables (i.e. $2 \mathrm{~N}$ ) are much smaller than the number of constraints, would reduce the number of superficial variables considerably. In short, solving the dual of the required LP problem, would save lots of computation time and storage requirements.

5.7 Properties of $\hat{S}_{\mathrm{X}}\left(\underline{z}_{\mathrm{L}}\right)$

The set $\hat{S}_{X}\left(\underline{z}_{L}\right)$, being defined by the intersection of $\hat{S}_{x}$ and $\hat{L}_{x}$, can be analyzed via LP. The following information can be obtained easily from $\hat{S}_{x}\left(\underline{z}_{L}\right)$ once it is formed.

1) Existance of secure solution:

The absence of a feasible solution satisfying the linear inequalities indicates that $\hat{\hat{S}}_{X}\left(\underline{z}_{L}\right)$ and hence that $S_{X}\left(\underline{z}_{L}\right)$ is 
empty. Note that existance of $\hat{\mathrm{S}}_{\mathrm{X}}\left(\underline{\mathbf{z}}_{\mathrm{L}}\right)$ does not necessary guarantee the existance of secure solutions.

2) Size and shape of $\hat{S}_{x}\left(\underline{z}_{L}\right)$ :

With the help of the algorithm proposed by Natheiss, [34], for an specific load distribution one can easily obtain all the vertices of the polytop which defines $\hat{S}_{X}\left(\underline{z}_{L}\right)$ and identify nonactive constraints. This data give an indication of the size and shape of $\hat{S}_{X}\left(\underline{z}_{L}\right)$ and sorts out the inequalities which define $S_{X}\left(\underline{z}_{L}\right) \operatorname{explicitly.~}$

3) Points inside $\hat{\mathrm{S}}_{\mathrm{X}}\left(\underline{z}_{I}\right)$ :

With a linear objective function, the only points accessible through the use of IP are vertices of the polytop that form $\hat{S}_{X}\left(\underline{z}_{L}\right)$. The fact that $\hat{S}_{X}\left(\underline{z}_{L}\right)$ is a close approximation to $\mathrm{S}_{\mathrm{X}}\left(\underline{z}_{\mathrm{L}}\right)$ tempt us to use these points for points belonging to $S_{x}\left(\underline{z}_{L}\right)$. These points are not, however, appropriate; since they represent conditions under which some of the linear constraints would be set at their upper or lower bounds. Furthermore such points, because of the approxmation inherent in their derivation, could cause slight violation of some of the original security constraints. This indicates that access to points which are inside $\hat{S}_{x}\left(\underline{z}_{L}\right)$ is essential.

Consider two vertices which do not belong to one hyperplane. The line connecting them lies entirely inside $\hat{S}_{x}\left(z_{I}\right)$. If all the vertices are found as suggested in [36] it is easy to pick up one such pair of points, otherwise one can obtain them by maximizing and minimizing a proper objective function over $\hat{S}_{x}\left(z_{L}\right)$. The points on the line are then given by 


$$
\underline{x}(t)=\underline{x}_{1}+t\left(\underline{x}_{2}-\underline{x}_{1}\right) \quad 0 \leqslant t \leqslant 1
$$

where $\underline{x}_{1}$ arid $\underline{x}_{2}$ are the above mentioned vertices. It practically necds no computational effort to insert (50) into constraints (9) to (12) and obtain the segment of the above line which satisfies all of them. Finally one can find the closest point to. the manifold $S_{X}\left(\underline{z}_{L}\right)$. by minimizing

$$
E(t)=\sum_{i=1}^{m} W_{P_{i}}\left[P_{i}^{l}-P_{i}(t)\right]^{2}+\sum_{i=1}^{m} W_{Q_{i}}\left[Q_{i}^{1}-Q_{i}(t)\right]^{2}
$$

subject to

$$
t_{3} \leqslant t \leqslant t_{b}
$$

where $t_{a}$ end $t_{b}$ mark the end points of the secure segment. $E(t)$ is an error function measuring the error of the real and reactive loads with those delivered at load buses for different points on the line. The weights $W_{P_{i}}$ and $W_{Q_{i}}$ are

$$
\begin{aligned}
& W_{P_{i}}=1 \frac{d P_{i}\left(t_{a}\right)}{d t_{a}}-\frac{d P_{i}\left(t_{b}\right)}{d t_{b}} \mid \\
& W_{Q_{i}}=1 \frac{d Q_{j}\left(t_{a}\right)}{d t_{a}}-\frac{d Q_{i}\left(t_{b}\right)}{d t_{b}} \mid
\end{aligned}
$$

and they merely reduce the impact of each term on the total crror depending on how flat the weighted function is over the range of interest, i.e. $\left(t_{a}, t_{b}\right)$. 


\subsection{Examples}

1) Two-bus system:

To enlighten some of the concepts discussed in the previous sections a test problem is treated here. The.system considered consist of a slack bus, a load bus and a transmission line, Fig. (5-5).

This case is, in particular, of some interest because it offeres a chance to develope a geometrical feeting of otherwise abstract concepts such as $S_{X}\left(\underline{z}_{L}\right)$ and $\hat{S}_{X}\left(\underline{z}_{L}\right)$. The employed data are listed in table (5-1).

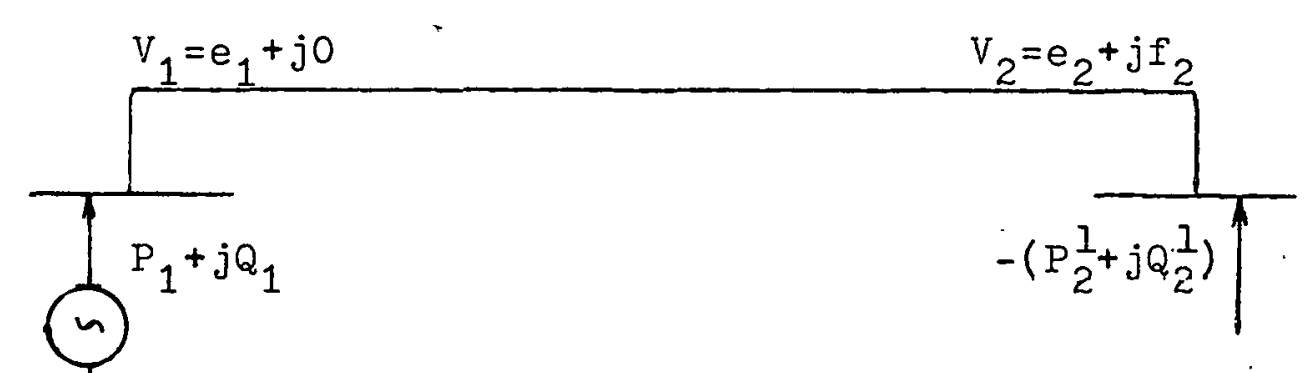

Fig. $(5-5)$

Table $(5-1)$

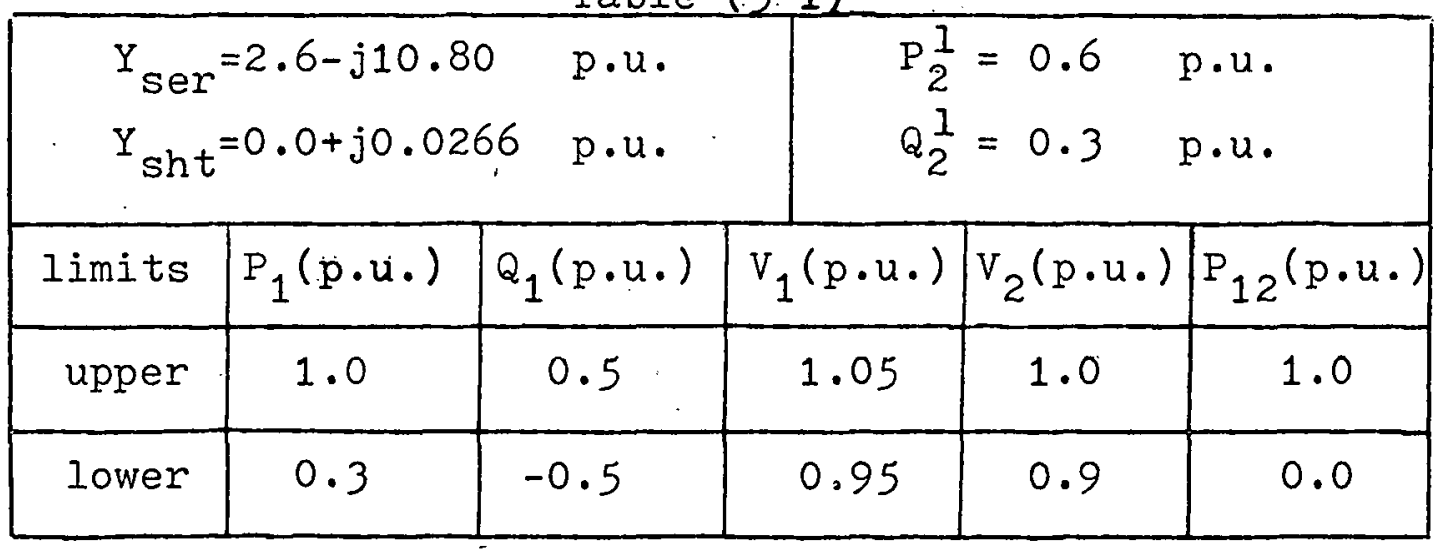




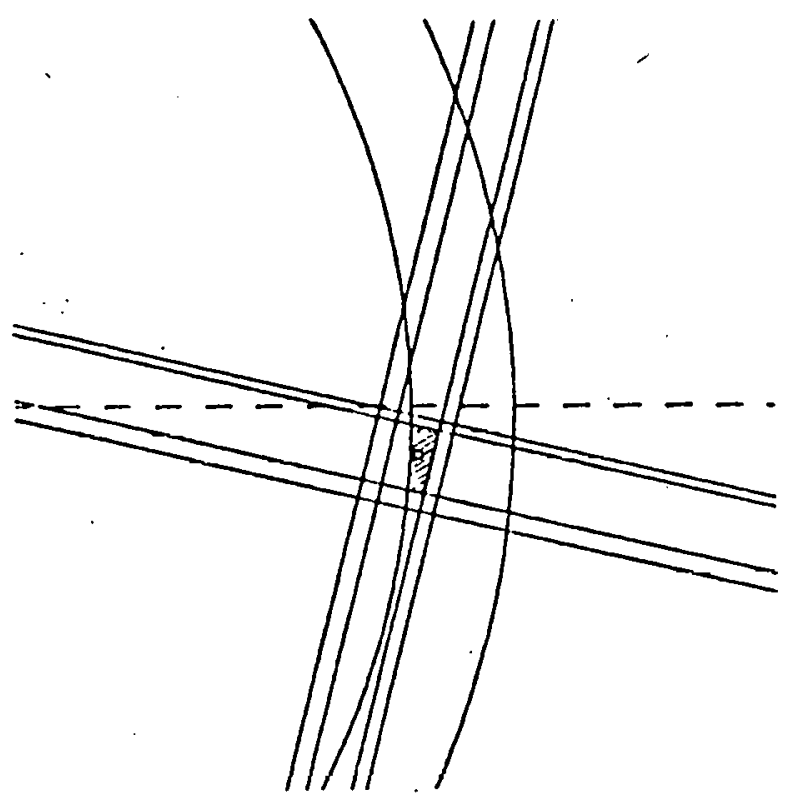

(a): $\quad e_{1}=0.95$

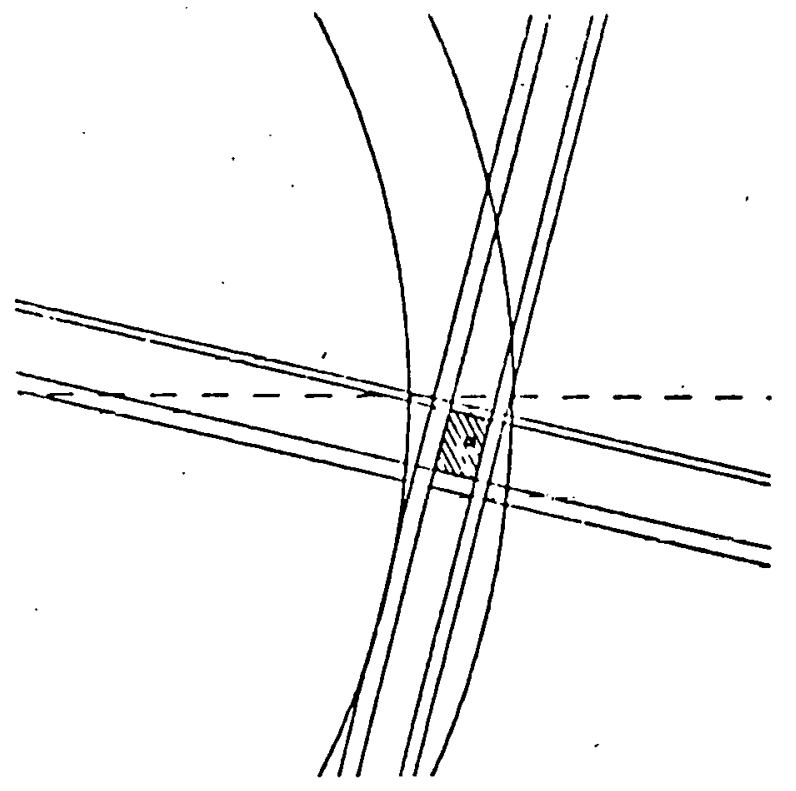

(b): $\quad c_{1}=1.00$

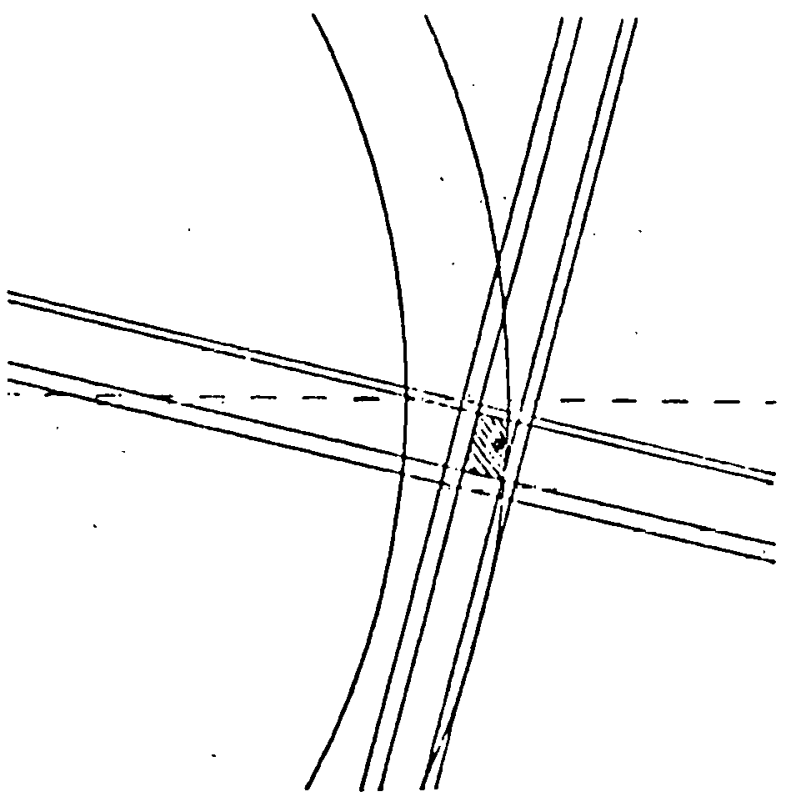

(c): $\quad e_{1}=1.05$

Fig. (5-6)- Different cross sections of $S_{x}$ and $S_{x}\left(\underline{z}_{L}\right)$ in $e_{2}-f_{2}$ plane (shadded). 
The shadded regions in figs. (5-6) are cross sections of the set of secure points, $S_{x}$, in $e_{2}-f_{2}$ plane for three different $e_{i}$. Note that $P_{1}, Q_{1}$, and $P_{12}$ are quadratic functions of $e_{1}$ and linear functions of $e_{2}$ and $f_{2}$. The set of secure solutions, $S_{X}\left(z_{L}\right)$, for this problem is that part of the intersection of the surfaces

$$
\begin{aligned}
& P_{2}^{I}=P_{2}(\underline{x}) \\
& Q_{2}^{I}=Q_{2}(\underline{x})
\end{aligned}
$$

(i.e. that part of $L_{x}$ ) which falls inside $S_{x}$. The small circles in figs. $(5-6)$ in the shadded region, represents crosssections of $\mathrm{S}_{\mathrm{X}}\left(\mathrm{z}_{\mathrm{L}}\right)$ for different $e_{1}$. Fig. (5-7) shows the entire plane $e_{2}-f_{2}$. This figure is added to indicate the relative size of the regions of interst.

linree cross-secliurs of $\hat{3}_{\mathrm{x}}$ are shown in fig. (5.8). Note that a reduction of about $50 \%$ in the number of the constraints is possible. Fig. (5-9) shows cross-sections of $\hat{S}_{X}\left(\underline{z}_{I}\right)$. The cross-sections of $S_{X}\left(\underline{z}_{I}\right)$, (the small circles), and the non-linear voltage constraints (the large circles) are included for comparison. Relative tight enclosement of $S_{X}\left(\underline{z}_{L}\right)$ by $\hat{S}_{X}\left(\underline{z}_{L}\right)$ is evident. Note that for the limits used in this example the binding constraints turn out to be only the four load dependant constraints of relations (41) and (42) plus the limits on the slack bus voltage, (45). 


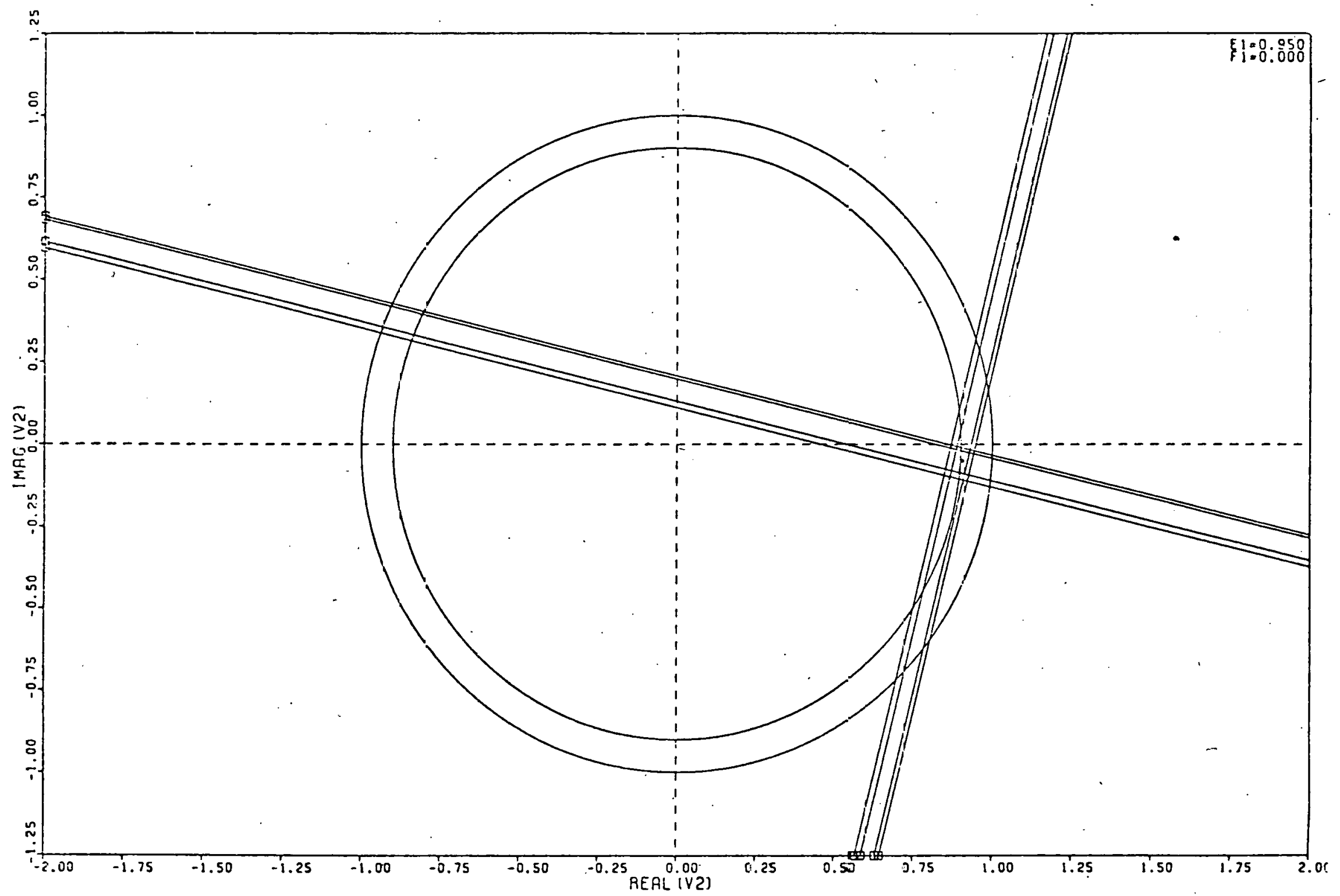

Fi $\tilde{s} \cdot(5-7)-A$ full cross-section of $S_{X}$ (and also $S_{X}\left(\underline{z}_{L}\right)$ to indicate the relative size of the non-linear set, $S_{x}$. 


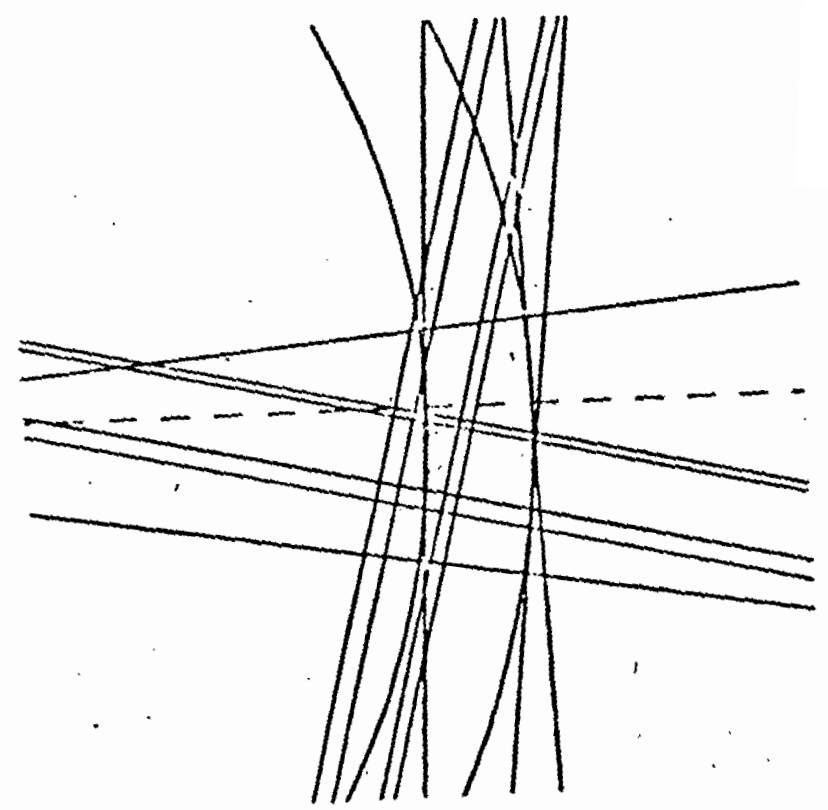

(a): $e_{1}=0.95$

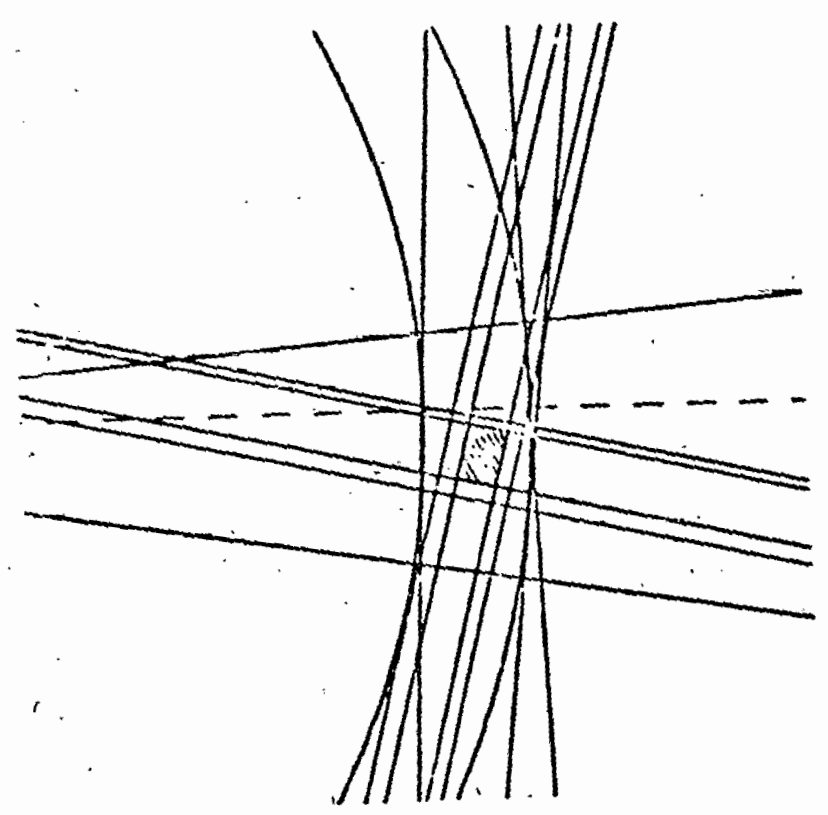

(b): $e_{1}=1.00$

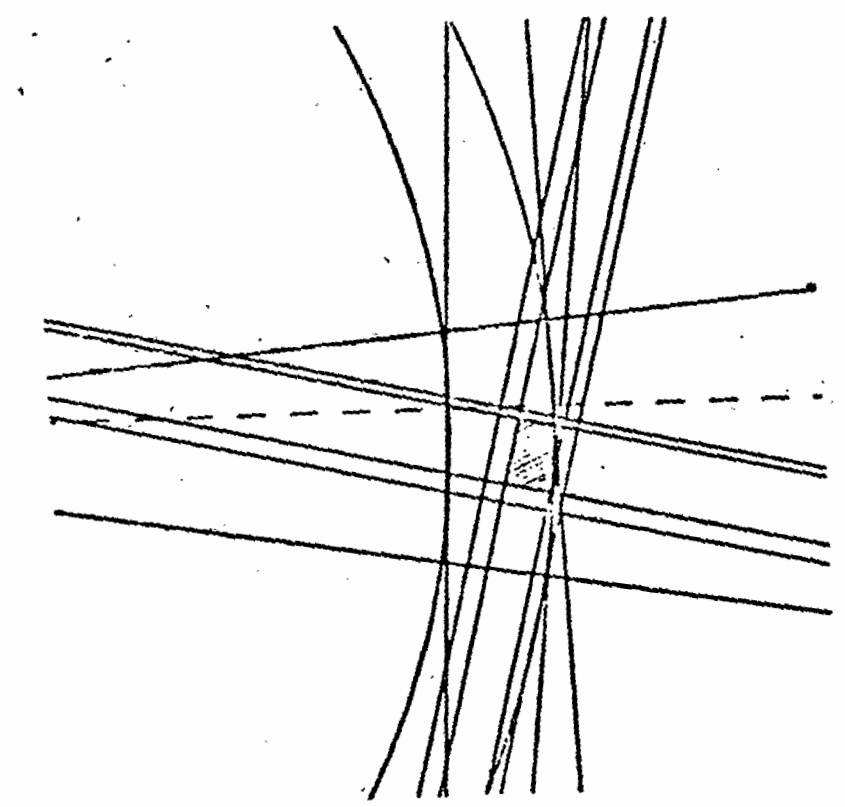

(c): $e_{1}=1.05$

Fig. (5-i) - Different cross sections of $s_{x}$ in $e_{2}-f_{2}$ plane. (The non-linear voltage constraints are included for comparison). 


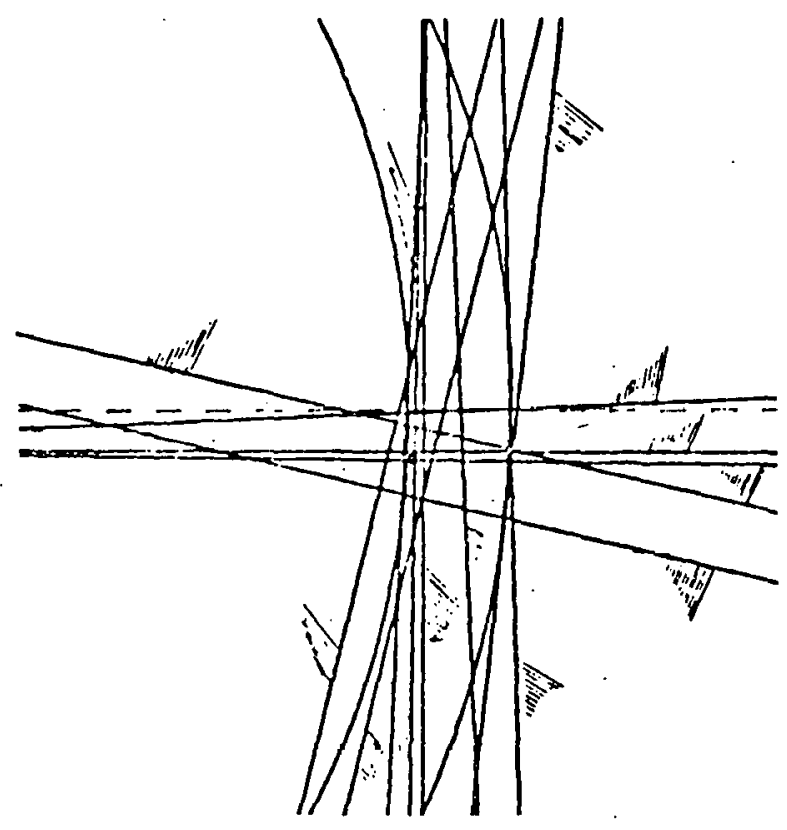

(a): $\quad e_{1}=0.95$

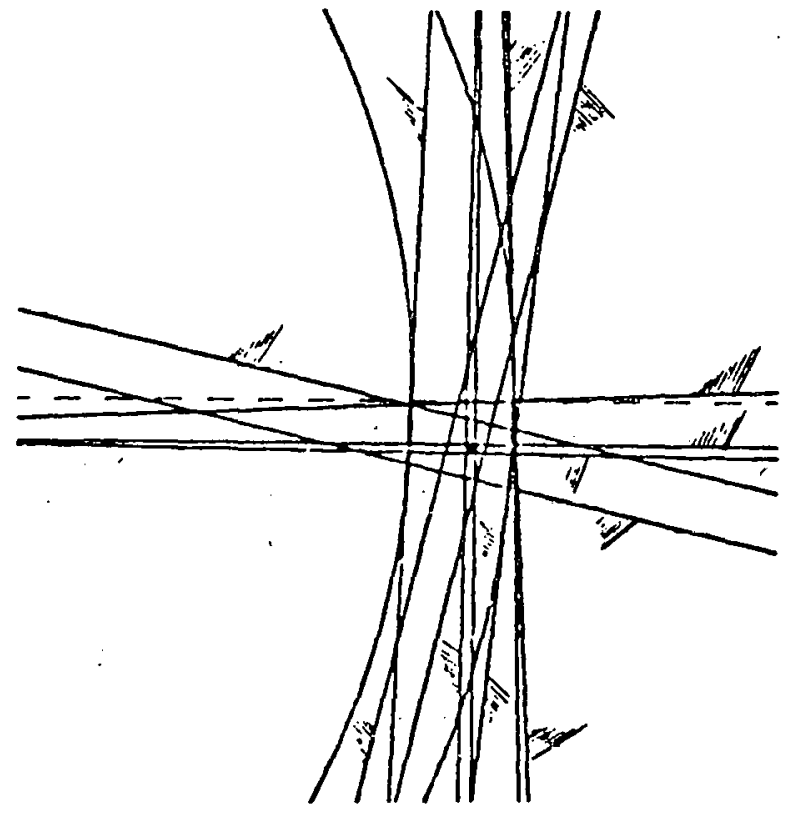

(b): $e_{1}=1.00$

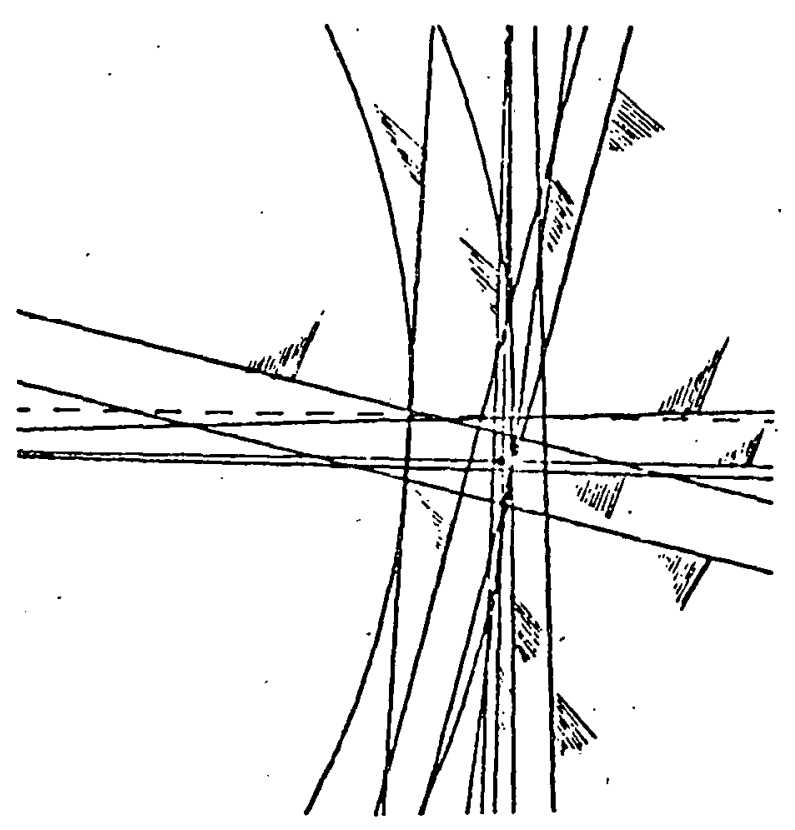

(c) $8 e_{1}=1.05$

Fig. $(5-9)$ - 'lhree cross-section of the linear set $\hat{\mathrm{S}}_{\mathrm{X}}\left(\underline{z}_{\mathrm{L}}\right)$ in $\mathrm{e}_{2}-\mathrm{f}_{2}$ plane. All linear constraints (active and inactive) plus croec-sections are $s_{x}\left(\underline{z}_{1}\right)$ and noin-linear voltage constraints are drawn. 
Some vertices of the polytop $\hat{\mathrm{S}}_{X}\left(\underline{z}_{I}\right)$. can be seen in parts (a) and (c) of fig. (5-9). An exagerated three dimensional picture of $S_{x}\left(\underline{z}_{L}\right)$ and $\hat{S}_{x}\left(\underline{z}_{L}\right)$ for this problem would look like the one shown in fig. $(5-10)$.

2) Eight-bus system

This system, fig. (5-11), is picked up from [7] and it represents a radial transmission of power from generation buses to the load buses with high degree of reliability. The data used in this example are given in the next page. To experiment with this system,

i) The real power demand at bus 8 is allowed to change.

ii) The thermal limits on the transmission lines are chosen such that there exist bottle-necks in the system regarding transmission of power.

iii) The power flow is considered to be only in one direction for each line.

iv) The auxilary limits (see Appendix B-5) are calculated neglecting dependance of powers on voltage magnitudes.

v) A simple " Gauss-iterative technique " load-flow is employed to check the results of the computer program developed to examine features of $\hat{\mathrm{S}}_{\mathrm{X}}\left(\underline{z}_{\mathrm{L}}\right)$.

Note that for this system'it is possible to have a change in the direction of power flow in a line as the load changes. In practice, however, at the transmission level this is unaccepltable since the Iine circuit-breakers in such case would 


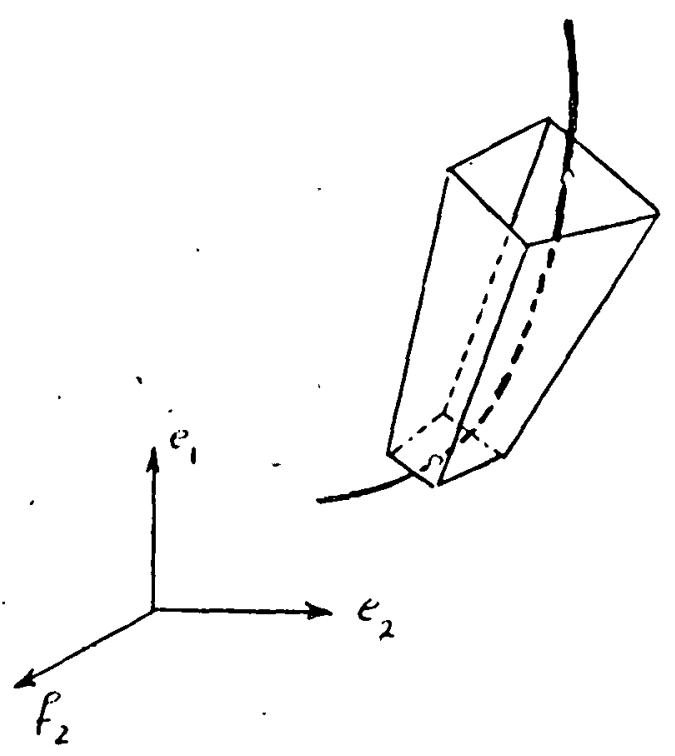

Fig. $(5-10)$ - An exagerated threp dimencional piclure of ${ }^{\prime}\left(z_{L}\right)$ and $\hat{S}_{X}\left(\underline{z}_{L}\right)$ for the two bus system.

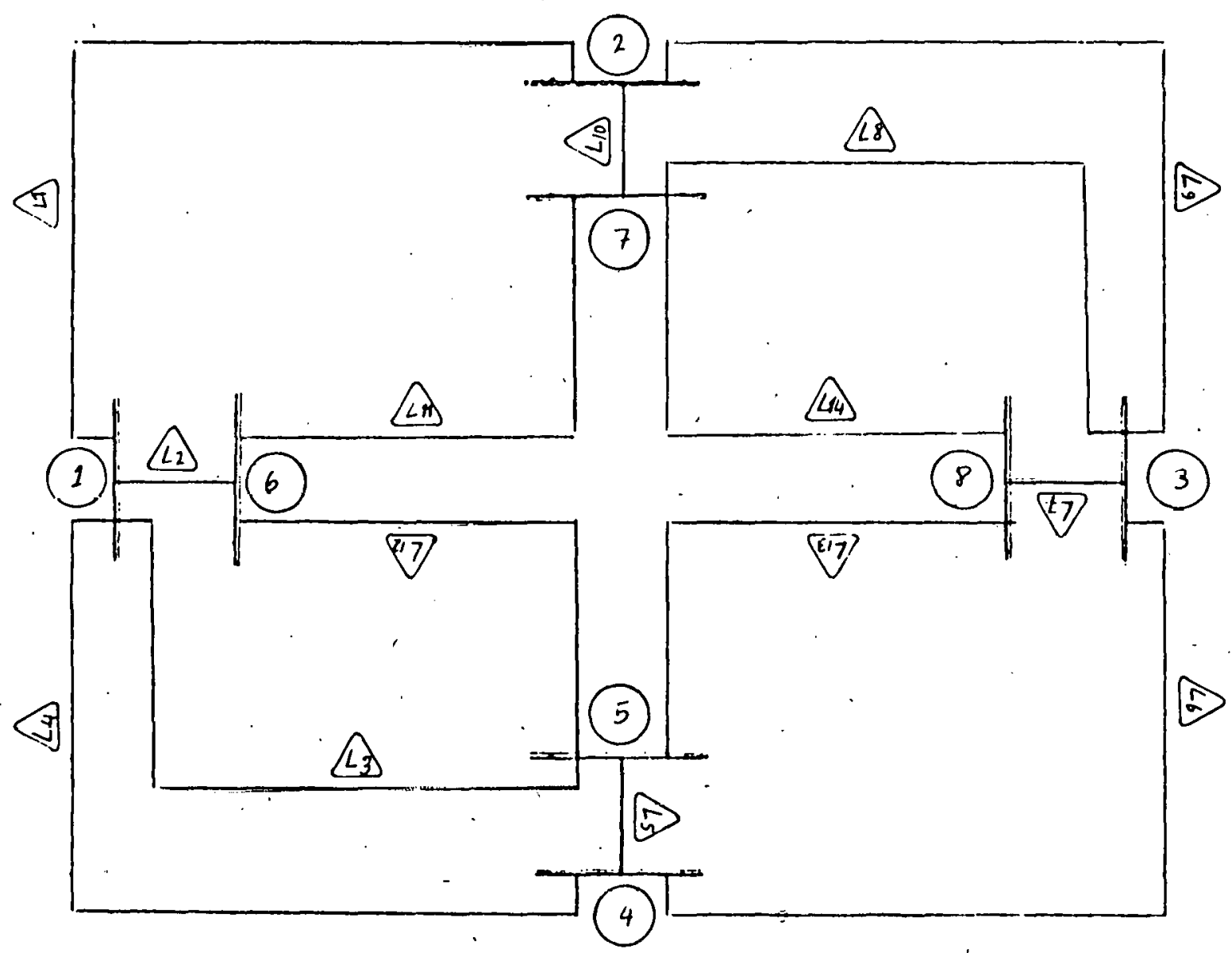

Fie. (5-11)- The eight bus system 
(INCLLDING SLLEKTES

$\infty$

$\stackrel{\infty}{\sim}$
AC. CF Da PUSES

LINE CATA

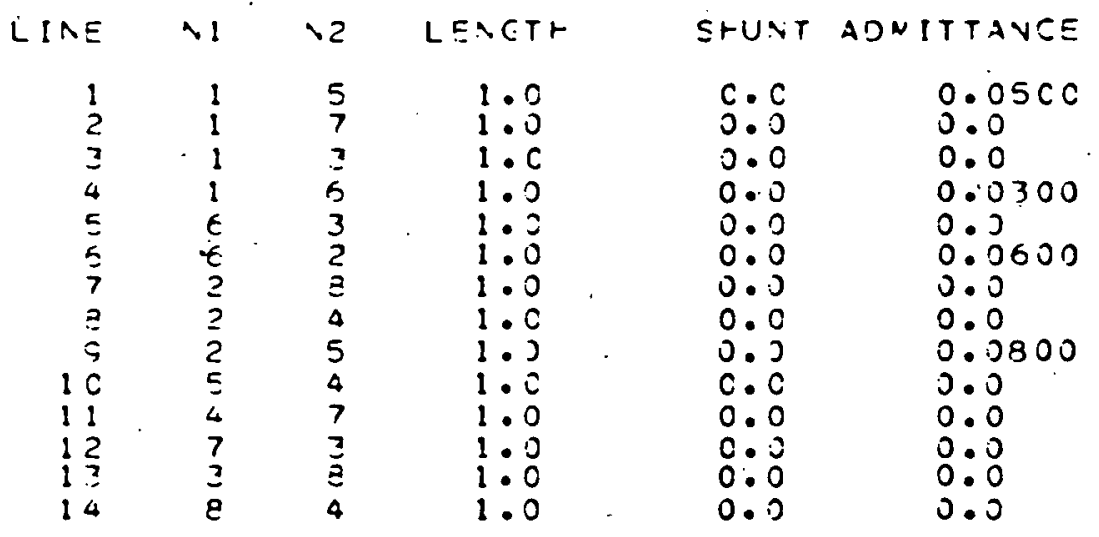

AC. CF ZERC LOAC BUSES

0
SERIES INFECANCE

$\begin{array}{ll}c .010 c & 0.07 c 0 \\ 0.002 c & c .0100 \\ 0.003 c & c .0300 \\ 0.002 c & c .0650 \\ 0.0035 & c .0200 \\ 0.0075 & c .0630 \\ 0.0070 & 0.0150 \\ 0.0010 & 0.0230 \\ 0.0025 & c .0230 \\ 0.0110 & c .0210 \\ 0.0032 & c .0300 \\ 0.0021 & c .0100 \\ 0.0020 & 0.01130 \\ 0.0016 & 0.0211 \\ 0.0021 & 0.0311\end{array}$

PV BUSES

VUIA

VNAX

D.M.I?

$F N \Delta X$

GNIN

$\cos x$

$\begin{array}{lll}1 & 2.93 & 1.02 \\ 2 & 0.95 & 1.05 \\ 3 & 1.00 & 1.10 \\ 0 & 0.00 & 1.00\end{array}$

1.00 .000
5.00000
5.20000
4.200200

$10.600=0$

$-5.00000$

s. 0000

2.3

1.06
$22 \cdot \operatorname{coccc}$

$25 . \operatorname{coc} 00$

20.50000
$-10 \cdot 00000$

$-5.00000$

$-5 \cdot 00000$
16.0200

$3 \mathrm{cosec}$

25.0000 


$\begin{array}{rrrrr}5 & 0.95 & 1.05 & -25.00000 & 10.00000 \\ 6 & 0.95 & 1.05 & -18.00000 & 2.50000 \\ 7 & 0.90 & 1.00 & -27.00000 & -13.00000 \\ 8 & 0.95 & 0.95 & -8.00000 & -10.0000 C\end{array}$

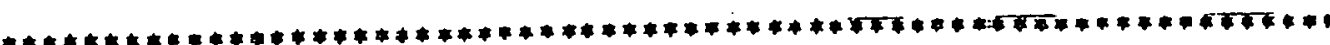

THERMAL LIMITS ( DCWER FLOW IS CONSIOERED TO EE FRCM BLS NI TOWARD BLS N2)

N2

15

17

31

16

36

26

28

24

25

45

47

37

03

84
PTMIN

$0.5000 \mathrm{C}$

$0.50 \mathrm{CCC}$

0.50000

c. 50000

3.00000

c. 50000

z. 50000

c. 50000

c. $5000 \mathrm{C}$

2.50000

2.50000

2. 00000

C. 50000

0.50000 ptmax

$$
\begin{array}{r}
10.00000 \\
5.00000 \\
5.00000 \\
2.50000 \\
15.00000 \\
5.0 .0000 \\
15.00000 \\
2.50000 \\
5.00000 \\
15.00000 \\
15.00000 \\
10.00000 \\
2.50000
\end{array}
$$$$
2.50000
$$ 
trip in response to a seemingly abnormal condition.

The following are different tests carried out on this systeria.

1) By settiner $\mathrm{l}_{3}=-10 \mathrm{p} . \mathrm{u} .$, the total real load exceeds $3 \mathrm{p} . \mathrm{u}$. ( $\left(4, \mu_{2}^{\prime}\right)$, the maximum generation capability of the system. It is clear that in this case $\mathrm{S}_{\mathrm{X}}\left(\underline{z}_{\mathrm{L}}\right)$ does not exist. The resulted LP tableau has no feasible solution indicating that $\hat{S}_{X}\left(\underline{z}_{L}\right)$ also does not exist.

2) The real load at bus 8 is set equal to zero. Now the maximum generation capacity is considerably larger than the demanded power and one expects $\hat{S}_{x}\left(\underline{z}_{L}\right)$ to exist. Here one can obtain a large set of feasible solutions from the LP tableau. The inside point evaluated here is a good condidate for a point belonging to $S_{x}\left(\underline{z}_{L}\right)$. The relatively small difference between feneration levels proposed by the LP output and that produced by the load flow and the rapid convereance of the load-flow are indications of this fact. This results are listed in $\Lambda$ ppendix $\mathrm{C}-5$.

3) When the real load at bus. 8 is increased to $-5 . p . u .$, the set $\hat{\mathrm{S}}_{x}\left(\underline{z}_{L}\right)$ disappears indicating again that the constrained load-flow would have no solution. Since the maximum generation capacity is much higher than the total load demand, it is clear that in this case the thermal limits are the binding constraints. Changing the thermal limits revealed that the low power handing capability of line 8 is one of the system bottle necks and here caused this problem. 
4) After changing the thermal limit of line 8 to 7.5 p.u., for a real power load demand of $-10 \mathrm{p} . \mathrm{u}$. , we run again into the same probiem, i.e. $\hat{\mathrm{S}}_{\mathrm{X}}\left(\underline{\mathrm{z}}_{\mathrm{L}}\right)$ disappears. This time the problem turns out to be the reversal of power flow in two of the lines.

5) The voltage linits on bus 8 are changed from $(0.95$ $1.05)$ to $(0.850 .95)$. Here, regardless of the amount of real power required at this bus, one is not able to form a nonempty $\hat{\mathrm{S}}_{\mathrm{X}}\left(\underline{\mathrm{z}}_{\mathrm{L}}\right)$.

\subsection{Conclusions.}

In this section we discussed a very simple and straightforward method for surrounding the very non-linear set of solutions to constrained load-flow, by a linear set. The simplicity of deriving the linear inequalities and the numerical results are quite encouraging. We believe that potentials of this approach is not yet fully explored and further investigations could bring into light a wide range of applications for it.

One of its possible applications is in planning and expansion of the power systems. As we saw, one can easily discover the bottle necks in a system and actually investigate the load distribution under which $\hat{S}_{X}\left(\underline{z}_{L}\right)$ and therefore $S_{x}\left(\underline{z}_{L}\right)$ will disappear. 
The points on the lines which connect two vertices of $\hat{S}_{X}\left(z_{L}\right)$ and fall inside $S_{X}$ are valuable set of points for optimal constrained load-flow runs. The techniques used in solving these type of problems require a feasible point as their starting point. This approach in fact suggests a systematic way for finding such points, eliminating an often frustrating trial and error procedure. 


\section{APPENDIX A-5}

The real power flowing from bus $i$ toward bus $j$ is, [7],

$$
P_{i j}=\frac{1}{R^{2}+X^{2}}\left[R V_{i}^{2}-R V_{i} V_{j} \cos \left(\theta_{i}-\theta_{j}\right)+X V_{i} V_{j} \sin \left(\theta_{i}-\theta_{j}\right)\right]
$$

where $R$ and $X$ are the line resistance and inductance respectively. Based on typical values that different variables and parameters in $(A-1)$ accept, the real power flow is well ap= proximated by,

$$
P_{i j} \simeq\left(\frac{v_{i} v_{j} X}{R^{2}+X^{2}}\right) \sin \left(\theta_{i}-\theta_{j}\right)
$$

Now consider the following inequalities

$$
\begin{gathered}
v_{i}^{m} \leqslant v_{i} \leqslant v_{i}^{M} \\
v_{j}^{m} \leqslant v_{j} \leqslant v_{j}^{M} \\
P_{1 j}^{m} \leqslant P_{i j} \leqslant P_{i j}^{M}
\end{gathered}
$$

One can use the above inequalities to arrive at

$$
\frac{P_{i j j}^{m}}{V_{i}^{M} V_{j}^{M}} \leqslant \frac{P_{i j}}{V_{i .} V_{j}} \leqslant \frac{P_{i j}^{M}}{v_{i}^{m} v_{j}^{M}} \quad\left(P_{i j}^{m}>0\right)
$$

By inserting equation $(A-2)$ into $(A-6)$, it becomes possible to establish bounds on $\sin \left(\theta_{i}-\theta_{j}\right)$, namely 


$$
\sin \delta_{i j}^{\grave{m}} \leqslant \sin \left(\theta_{i}-\theta_{j}\right) \leqslant \sin \delta_{i j}^{\mathbb{M}}
$$

where

$$
\begin{aligned}
& \sin \delta_{i j}^{m}=\left(\frac{R^{2}+X^{2}}{X}\right) \frac{P_{i j}^{m}}{V_{i}^{M} v_{j}^{M}} \\
& \sin \delta_{i j}^{M}=\left(\frac{R^{2}+X^{2}}{X}\right) \frac{P_{i j}^{M}}{v_{i}^{m} v_{j}^{m}}
\end{aligned}
$$

Assuming $\left|\theta_{i}-\theta_{j}\right| \leqslant 90^{\circ}$, from equation (A-7) it follows

$$
\delta_{i j}^{m} \leqslant \theta_{i}-\theta_{j} \leqslant \delta \delta_{i j}^{M}
$$

Noting that the voltage angle at the reference bus, $\theta_{1}$, is specified, for those buses which are connected to it, one has

$$
\theta_{1}+\delta_{\mathrm{k} 1}^{\mathrm{m}} \leqslant \theta_{\mathrm{k}} \leqslant \theta_{1}+\delta_{\mathrm{k} 1}^{\mathrm{M}}
$$

The ahove bounds on $\theta_{k}$ are of the form

$$
\theta \underset{\mathrm{k}}{\mathrm{m}} \leqslant \theta \quad \mathrm{k} \leqslant \theta_{\mathrm{k}}^{\mathrm{M}}
$$

and they can be used to find similiar bounds on those buses which are connected to the buses which are connected to the reference bus, i.e. we add $(A-12)$ to

$$
\delta_{i k}^{m} \leqslant \theta_{i}-\theta_{k} \leqslant \delta_{i k}^{M}
$$

to get

$$
\delta_{i k}^{m}+\theta \underset{k}{m} \leqslant \theta_{i} \leqslant \theta_{k}^{M}+\delta_{i k}^{M}
$$

and so on. 


\section{APPENDIX B-5}

To find the bounds on $\left(\frac{P_{i}}{V_{i}^{2}}\right)$ and $\left(\frac{Q_{i}}{V_{i}^{2}}\right)$ one has to solve the following problem

$$
\text { Maximize or Minimize } \frac{z_{i}(\underline{x})}{v_{i}^{2}(\underline{x})} \quad i=1, \ldots, 2 n
$$

subject to

$$
\begin{gathered}
z_{i}^{m} \leqslant z_{i}(\underline{x})=\underline{x}^{T}\left[J_{i}\right] \underline{x} \leqslant z_{i}^{M} \\
\left(v_{i}^{m}\right)^{2} \leqslant v_{i}^{2}(\underline{x})=\underline{x}^{T}\left[E_{i}\right] \underline{x} \leqslant\left(v_{i}^{M}\right)^{2}
\end{gathered}
$$

where $Z_{i}(\underline{x})$ represents $P_{i}(\underline{x})$ or $Q_{i}(\underline{x})$ and $\left[J_{i}\right]$ and $\left[E_{i}\right]$ are sparse, real, symmetric matrices.

After finding limits, for example, on $\frac{z_{1}(x)}{v_{1}^{2}}$, these newly established inequalities should join that of ${ }^{1}(B-2)$. This should continue till $i=2 n$, where one starts again setting limits on $\frac{z_{1}(\underline{x})}{v_{1}^{2}}$, with all the newly established inequalites, except two, included in $(B-2)$. This procedure must go on till the bounds on $\frac{z_{i}(x)}{v_{i}^{2}} \quad(i=1, \ldots, 2 n)$ stablize.

For large power systems, it is clear that the dimension and complexity of this problem would be enormous. However, with rapid developement in the area of computing speed and memory expansion of digital computers, solving such problems would not be a major undertaking in near future. 
The fact that we intend to surround $S_{X}\left(\underline{z}_{L}\right)$ by $\hat{\vec{S}}_{X}\left(\underline{z}_{L}\right)$ from above, on the other hand, allows us to seek other methods which may trade-off simplicity for accuracy. One such method is the following:

Let us assume that $z_{i}(\underline{x})$ and $v_{i}^{2}(\underline{x})$ are not related to each other. In this case it is easy to have

where

$$
L_{i}^{m} \leqslant \frac{Z_{i}}{v_{i}^{2}} \leqslant L_{i}^{M} \quad i=1, \ldots, 2 n
$$

$$
L_{i}^{M}=\frac{z_{i}^{M}}{\left(v_{i}^{m}\right)^{2}} \text { or } \frac{z_{i}^{M I}}{\left(v_{i}^{M}\right)^{2}}
$$

and

$$
I_{i}^{m}=\frac{z_{i}^{m}}{\left(v_{i}^{\sqrt{n}}\right)^{2}} \text { or } \frac{z_{i}^{m}}{\left(v_{i}^{m}\right)^{2}}
$$

Bepending on the sign of $z_{i}^{m}$ and $z_{i}^{M}$, from different values for $I_{i}^{M / I}$ and $L_{i}^{m}$, those should be chosen that maximize $\left|L_{i}^{M}-L_{i}^{m}\right|$.

This approach provides conservative limits on $\frac{Z_{i}(\underline{x})}{v_{i}^{2}(\underline{x})}$, but the margin of error is not large. This is in particular true for $z_{i}(\underline{x})=P_{i}(\underline{x})$, because the real power injections are quite insensitive to voltage magnitude variations.

APPENDIX $\quad C-5$

The equations of lines 1 and $2(f i g \cdot(5-4))$ in the $e_{i}-f_{i}$ plane are given by: 


$$
\begin{aligned}
& \left(\sigma k_{1}-\rho^{\prime} k_{2}\right) e_{i}+\left(\rho k_{1}+\sigma k_{2}\right) f_{i}+h\left(k_{1}, k_{2}\right)=0 \\
& \left(\sigma^{\prime} k_{1}-\rho^{\prime} k_{2}\right) e_{i}+\left(\rho^{\prime} k_{1}+\sigma^{\prime} k_{2}\right) f_{i}+h^{\prime}\left(k_{1}, k_{2}\right)=0
\end{aligned}
$$

where

$$
\begin{aligned}
& \sigma=d_{i}^{M}\left(B_{i i} Q_{i}^{I}-G_{i i} P_{i}^{I}\right)+1 \\
& \rho=d_{i}^{M}\left(B_{i i} P_{i}^{I}+G_{i i} Q_{i}^{I}\right) \\
& d_{i}^{M}=\left(\frac{V_{i}^{M}}{s_{i}^{I}}\right)^{2}
\end{aligned}
$$

and $h_{i}\left(k_{1}, k_{2}\right)$ is a function of $k_{1}, k_{2}$, and all voltage components except $e_{i}$ and $f_{i}$. When $d_{i}^{M}$ is replace by

$$
d_{i}^{m}=\left(\frac{V_{i}^{m}}{s_{i}}\right)^{2}
$$

in $(C-1)$ and $(C-2)$, then $\sigma, \rho$, and $h\left(k_{1}, k_{2}\right)$ change to $\sigma^{\prime}, \rho^{\prime}$, and $h^{\prime}\left(k_{1} k_{2}\right)$. The lines 3 and 4 are given by the same expressions, $(\mathrm{C}-1)$ and $(\mathrm{C}-2)$, but $\mathrm{k}_{1}$ and $\mathrm{k}_{2}$ are replaced by $k_{3}$ and $k_{4}$ respectively. To minimize the shadded area first we examine the angle between the lines 1 and 2 .

It can be easily proved that

$$
\tan \left(\theta_{12}\right)=\frac{\sigma \rho^{\prime}-\sigma^{\prime} \rho^{\prime}}{\sigma \sigma^{\prime}+\rho \rho^{\prime}}
$$

This angle is independent of $\mathrm{k}_{1}$, and $\mathrm{k}_{2}$ which indicates that the shadded area depends soley on the angles that the lines 
1 and 2 make with the lines 3 and 4 . Considering variation of the shadded area with these angles, one can easily arrive at the conclulsion that the minimum area happens when the angle which the line 1 makes with the line 3 (and thus the angle which the line 2 makes with the line 4 ) becomes $90^{\circ}$. In this case one should have

$$
\left(\frac{\sigma k_{1}-\rho k_{2}}{\rho k_{1}+\sigma k_{2}}\right)\left(\frac{\sigma k_{3}-\rho k_{4}}{\rho k_{3}+\sigma k_{4}}\right)=-1
$$

Equation (C-7) simplifies to

$$
k_{1} k_{3}+k_{2} k_{4}=0
$$

or

$$
\left(\frac{k_{1}}{k_{2}}\right)\left(\frac{k_{3}}{k_{4}}\right)=-1
$$

This means that the lines which have $\left(k_{1}, k_{2}\right)$ and $\left(k_{3}, k_{4}\right)$ as their coefficients should themselves be perpendicular.

\section{APPENDIX D-5}

(See the following pages) 
VALUE TE THE CEJECTIVE FUNETIDN

1.90500
CFROA COC:

0

\section{COST FUACTION COFFFICIENTS:}

$1 \quad-1.000 \quad-1.000 \quad-1.000 \quad-1.000$

VALUE OF THE DEJECTIVE FUNCT:ON

ERAR CחNE

1.97700

0 $x \mathbf{l}$

$\begin{array}{rr}0.95000 & 0.00300 \\ 0.90561 & -0.04761\end{array}$ $\times 2$

$1.03000-0.00000$
$0.09146-0.04446$ 


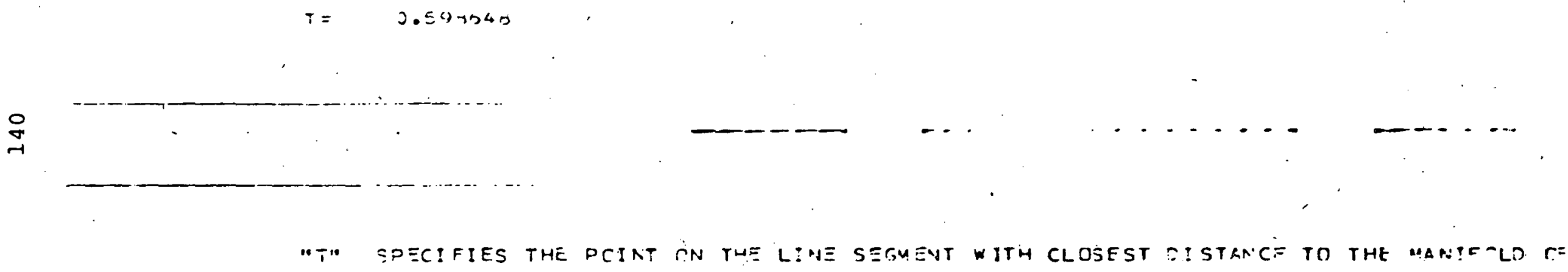

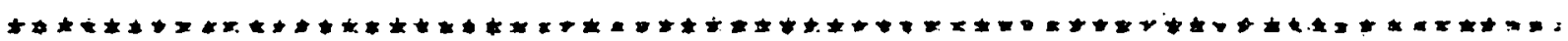

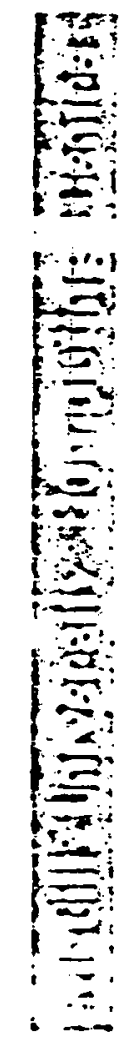




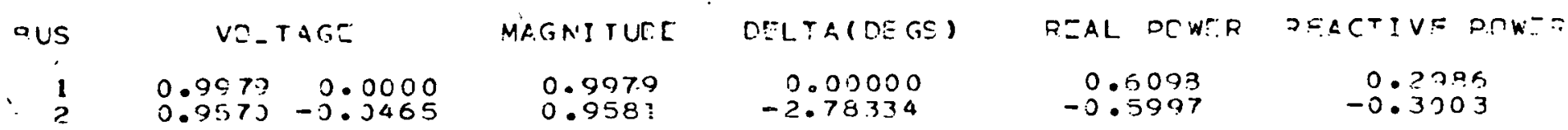

$\begin{array}{ccccc}\text { LINE } & \text { N1 } & \text { N? } & \text { FEAL POWEF } & \text { CEACTIVE POWE } \\ 1 & 2 & 1 & -0.5987 & -0.2579 \\ 1 & 1 & 2 & 0.6098 & 0.34 .36\end{array}$

GAUSS ITEFATIV́E TESHNI OUE CONVEFGSC IN 1 ITERETIGNS

SYSTEM INJECTIONS CALCULATEO FOR THE GIVEN CONTROL VARIAELES

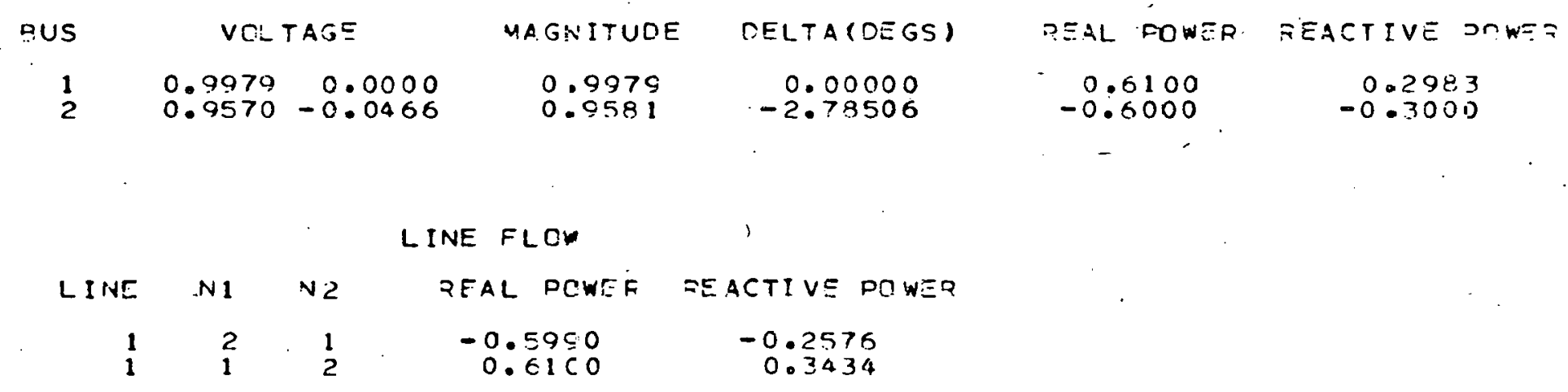




\subsection{SECURITY REGION IN THE SPACE OF INJECTIONS}

\subsection{Motivation}

The constraints defining the security region are explicit

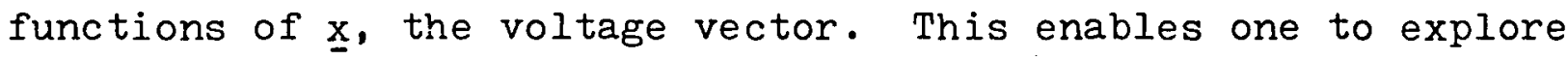
certain characteristics of the security region in the voltage space, $S_{x}$, with relative ease. The security region in the voltage space, however, is not suitable for many aspects of security and control enhancement. To simply detect a vulnerable condition one needs to run a load flow which, disregarding the possibility of divergence, is by itself a time consuming step. On the other hand, once the security region in the injection space, $S_{z}$, is characterized, detection of such conditions ís relatively simple and fast.

- The boundary of $\mathrm{S}_{2}$ is a mapping of the boundary of $\mathrm{S}_{\mathrm{x}}$ into the injection space, through a set of non-linear algebraic equations known as the load-flow equations. To characterize $\mathrm{S}_{\mathrm{z}}$, first one needs to have simple, explicit analytical expressions for the hyper-surfaces which form its boundary. To move in this direction, we have tried to formulate a general, systematic transformation which maps hyper-surfaces from the voltage space into the space of injections. The advantage of developing such transformation is two-fold:

1) One can transform all the constraints from the voltage space into the injection space and express them explicitly 
in terms of $\underline{z}$, the injection vector. This allows one to examine the possibility of explicit characterization of $\mathrm{S}_{\mathrm{z}}$.

2) One can use this transformation to map a certain part of $\mathrm{S}_{\mathrm{x}}$ into the injection space. This approach - which is quite original - permits one to surround a desirable operating point, for example, with a secure sphere in the voltage space, and then find its corresponding image in the space of injections.

In power-systems, not all the constraints are in general active. Filtering out the redundant (inactive) constraints is considered to be an essential part of characterization of $S_{z}$. Techniques such as the one just explained can be used successfully for this purpose.

\subsection{Transformation Formulae}

Transformation of the hyper-surfaces, from the voltage space into the injection space, is carried out through a Taylor series expansion of the defining dependent variables in terms of $\underline{z}$. Let $Y$ represent a dependent variable, then

$$
\underline{Y}=G(\underline{x})
$$

wher $G$ is an explicit function of the voltage vector; $x$. The injection vector, $\underline{z}$, is related to $x$ via the load-flow equations, i.e. a set of non-linear algebraic equations of the form

$$
\underline{z}=\underline{F}(\underline{x})
$$


The explicit nature of $G(\underline{x})$ and $\underline{F}(\underline{x})$ allows one to formulate a Taylor series expansion of $Y$ in terms of $\underline{z}$, that is

$Y=Y\left(\underline{x}_{0}\right)+\left(\frac{\partial \underline{Y}}{\partial \underline{z}}\right)_{\underline{X}=\underline{x}_{0}} \Delta \underline{z}+\frac{1}{2} \Delta \underline{z}^{T}\left(\frac{\partial^{2} \underline{Y}}{\partial \underline{z}^{2}}\right)_{\underline{X}=\underline{x}_{0}} \Delta \underline{z}+\cdots$

where $\Delta \underline{z}=\underline{z}-\underline{z}\left(\underline{x}_{0}\right)$ and $\underline{x}_{0}$ is the expansion point.

Various approximation formulae can be derived on the basis of (3). A Iinear approximation to $Y$, denoted by $Y_{1}$, is simply the first two terms of (3). It is shown in Appendix A-6 that

$$
Y_{1}=\underline{\beta}^{T} \underline{z}
$$

where $\underline{\beta}$, a $2 N-1$ dimensional vector, is a function of $\underline{x}_{0}$ and the network parameters. Approximating $Y$ by the first three terms of (3) produces a quadratic formula for $Y$, denoted by $\mathrm{Y}_{2}$. From. Appendix $A-6$,

$$
\underline{Y}_{2}=\underline{\beta}^{\mathrm{T}} \underline{\mathrm{z}}+\underline{\mathrm{z}}^{\underline{\mathrm{T}}}[\mathrm{C}] \underline{\mathrm{z}}
$$

where $[\mathrm{C}]$ is a $(2 \mathrm{~N}-1) \times(2 \mathrm{~N}-1)$, real, symmetric matrix which also varies with $\underline{x}_{0}$ and the network parameters.

In Appendix A-6, the accompanying paper, the following properties for these transformation formulae are éstablished:

1) The linear expansion of $Y, Y_{1}$, though much less accurate than the higher order expressions, is sufficiently accurate to approximate $Y$ closely over a wide range of $x$.

2) The quadratic term in $Y_{2}$ is essentially a first order 
error correction to $Y_{1}$ and improves the accuracy of $Y_{2}$ over $Y_{1}$ to a large extent. The quadratic expansion of almost all different types of constraints are quite accurate and reliable.

3) A higher order approximation to $Y$ is also possible. The accuracy will be increased at the expense of solving a system of linear equations, with the factorized Jacobian of the system as the coefficient matrix, per term.

The linear approximation of dependent variables, because of their simplicity and susceptibility to linear programming has always been attractive to researchers in this field. Many techniques have been developed to establish linear relations between dependent variables and nodal injections and a great deal of research has been centered around the utilization of such linear approximation in security and/or economic dispatch. The recent paper by B. Stott and J.L. Marinho, " Linear programming for power-system network security applications " is one such example of current efforts. Unfortunately the linearization techniques employed in these published works lack the generality required for such applications. The injection vector, almost without exception, does not contain the voltage levels at PV buses, and the constraints which are considered are usually limited to bounds on branch flows. This essentially deprives the resulting algorithm.. from systematic manipulation of voltage levels at $\mathrm{PV}$ buses to avoid violation of reactive generation limits and voltage level bounds at load buses. 
The linear expansion formulae developed here do not posess any of these draw-backs and pave the way for developing a broad algorithm of this kind. Moreover, extensive numerical runs on the linear expansion formulae have proved that their accuracy is far better than the other linear versions.

The specific application of linear and quadratic expansions of constraints considered here, i's their use in explicitly describing $S_{z}$ or part of $S_{z}$. This is the subject of the next sub-sections.

\subsection{Explicit Characterization of $\mathrm{S}_{2}$}

A two bus system is chosen to demonstrate. $S_{x}$ and $S_{z}$ and their linear and quadratic approximations, figs. $(6-1)$ to $(6-5)$. Even for such a simple example, the extreme non-linearity and complexity of $\mathrm{S}_{\mathrm{z}}$ is appreciable. An explicit analytic description of $\mathrm{S}_{\mathrm{z}}$ is obviously an almost impossible task. But the question is: does one really need to describe the whole $\mathrm{S}_{\mathrm{z}}$ ? We know that in a power-system the variations of the injection vector, $\underline{z}$, under normal conditions are quite gradual, and it could stay practically about the same operating point for hours. In this case, the dispatcher would like:

1) To have a simple, explicit function to describe that part of $\mathrm{S}_{\mathrm{z}}$ which surrounds the operating point. 


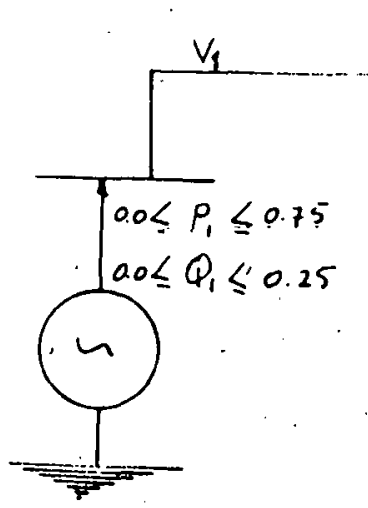

$0.95 \leqslant v_{2} \leqslant 1.05$

Fig. $(6-1)$ - lihe two bus system for which $\mathrm{S}_{\mathrm{X}}$ and $\mathrm{S}_{\mathrm{Z}}$ arc shown in figs. $(6-2)$ and $(6-3)$.

Fig. $(6-2, a, b, c)$ - Three cross-sections oi the jet of secure voltures, is $x^{\circ}$
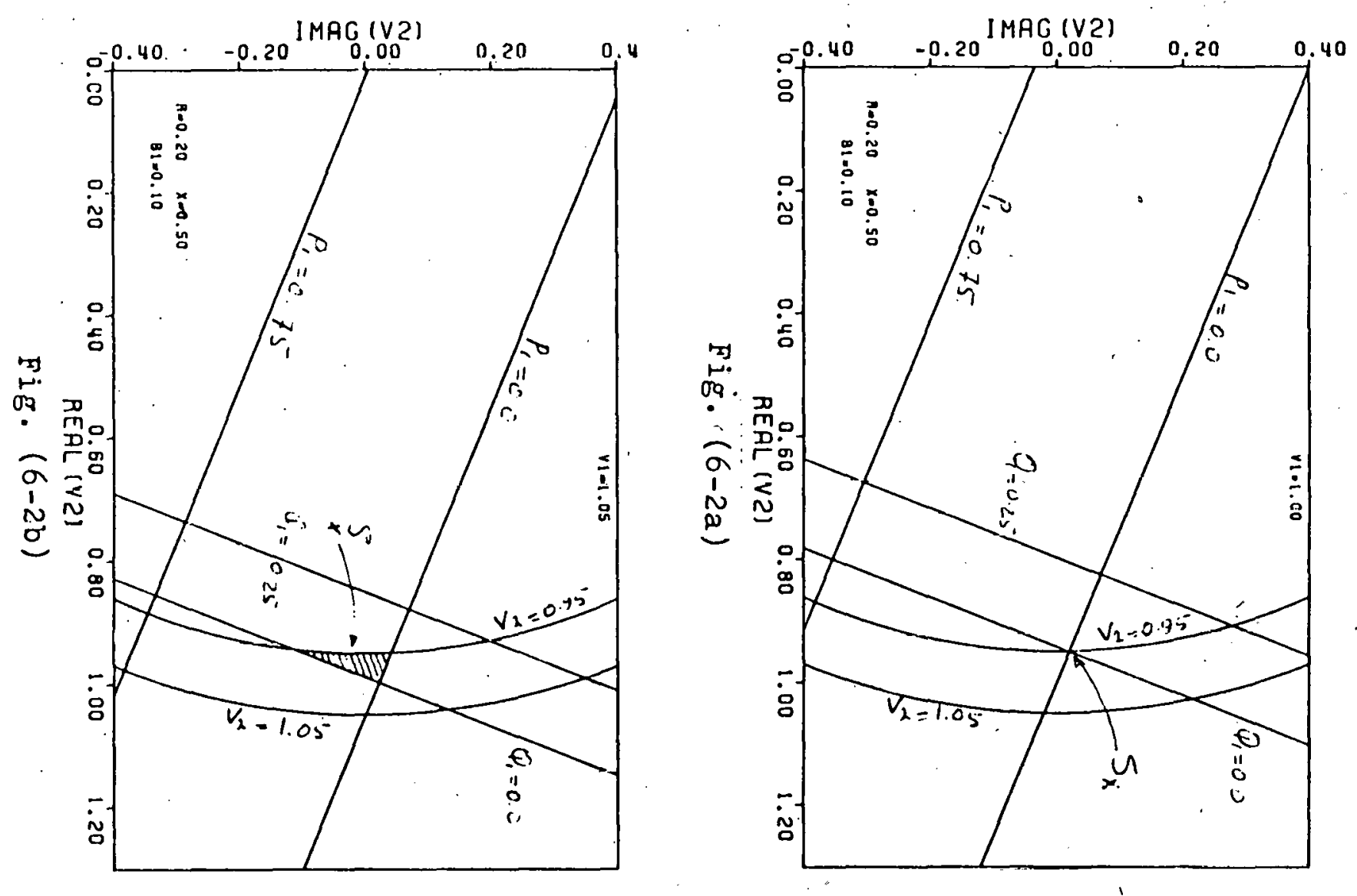


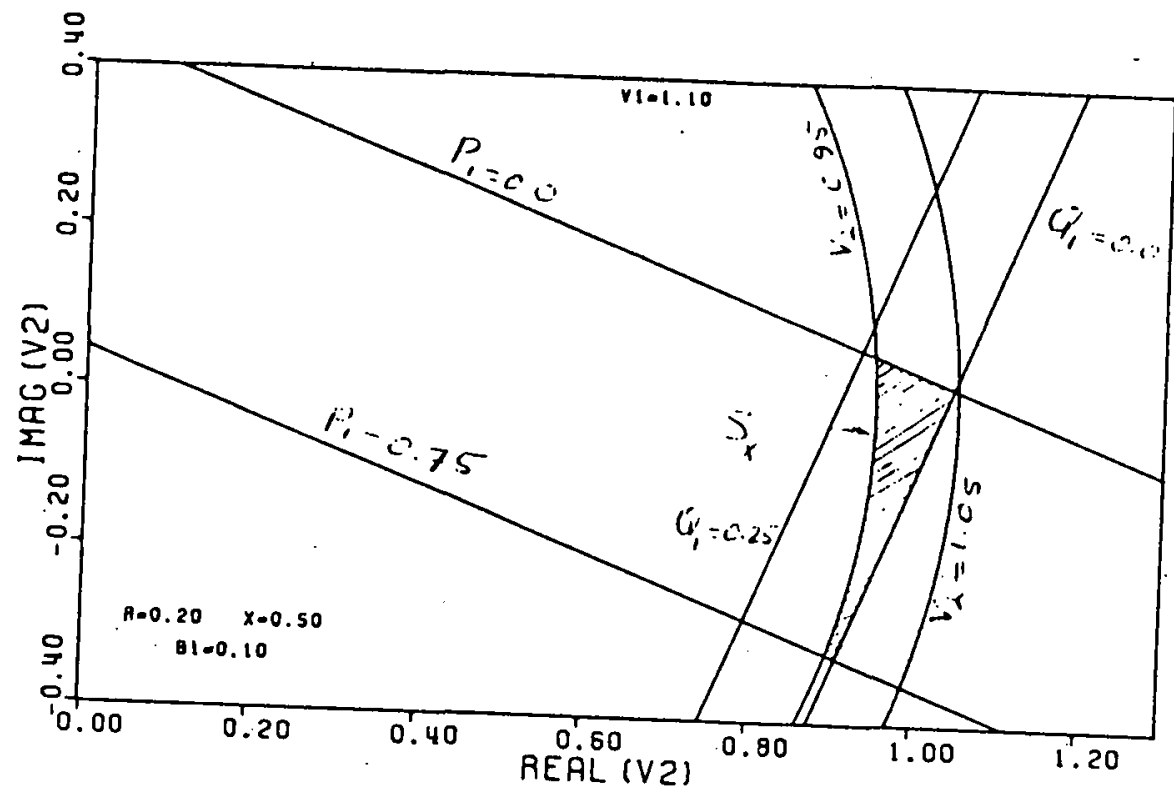

Fig. $(6-2 c)$

Fi.c. $(6-3, a, b, c)$-Three cross-sections of the set of secure iniections, $\mathrm{S}_{\mathrm{z}}$.

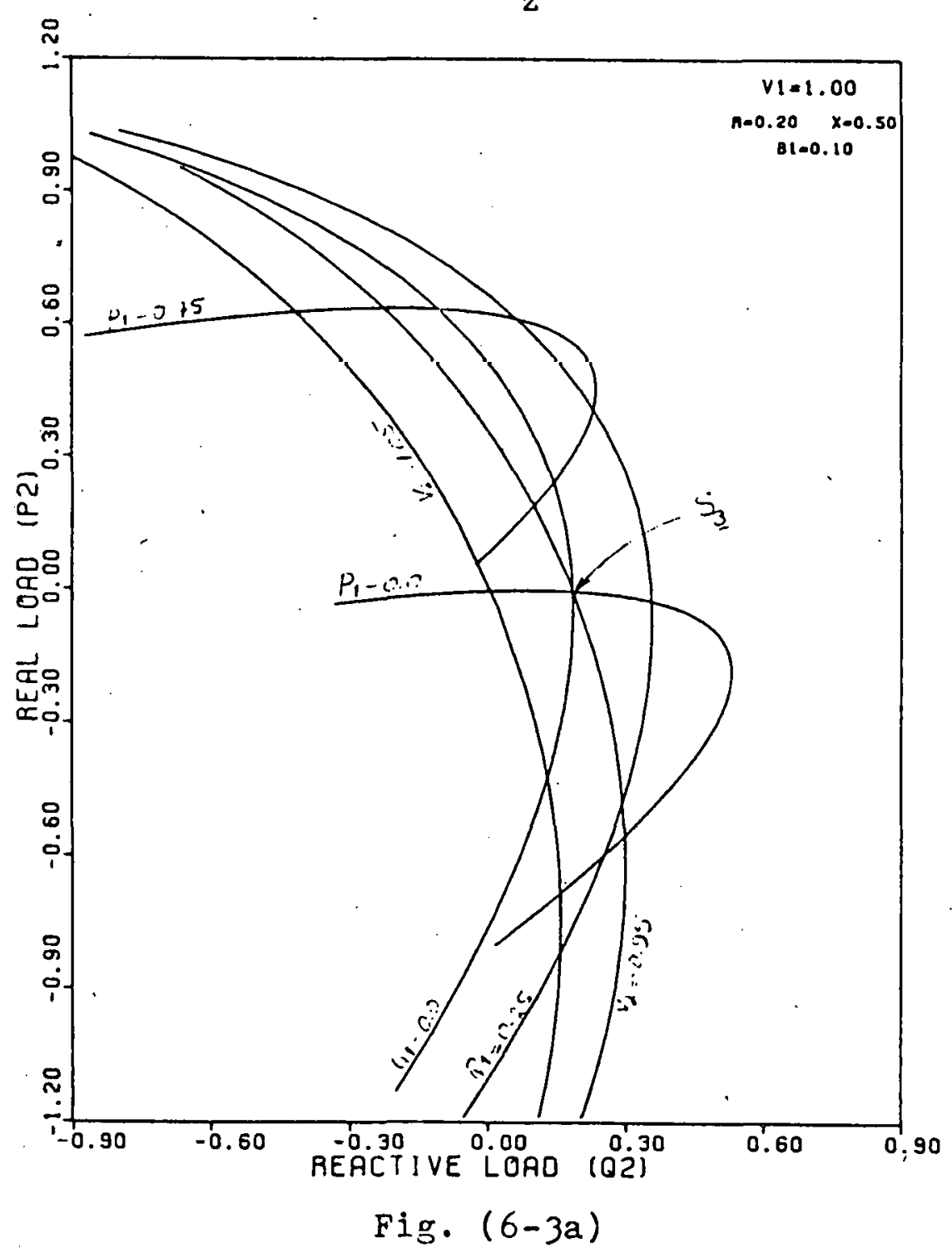




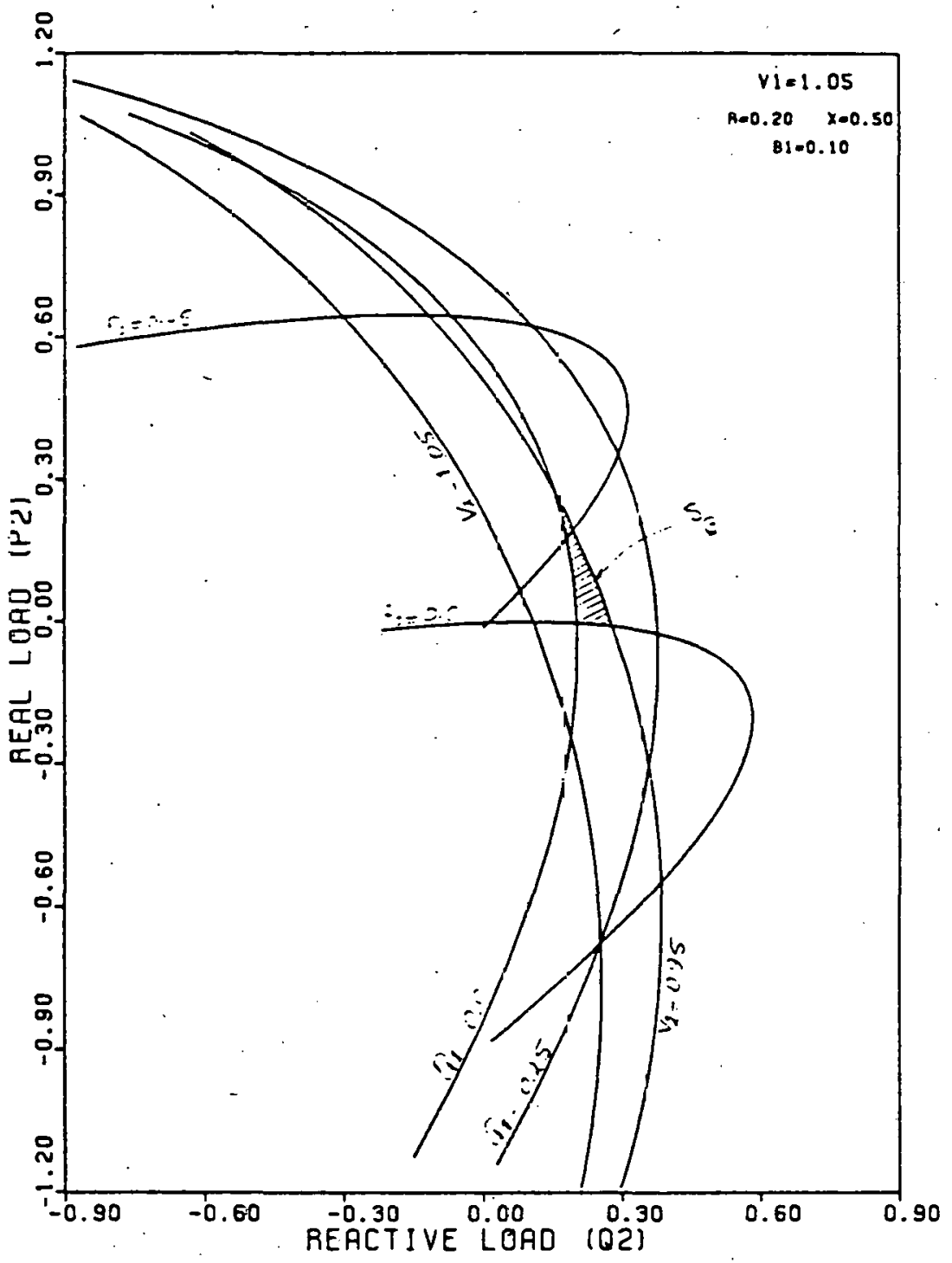

Fig. $(6-3 b)$

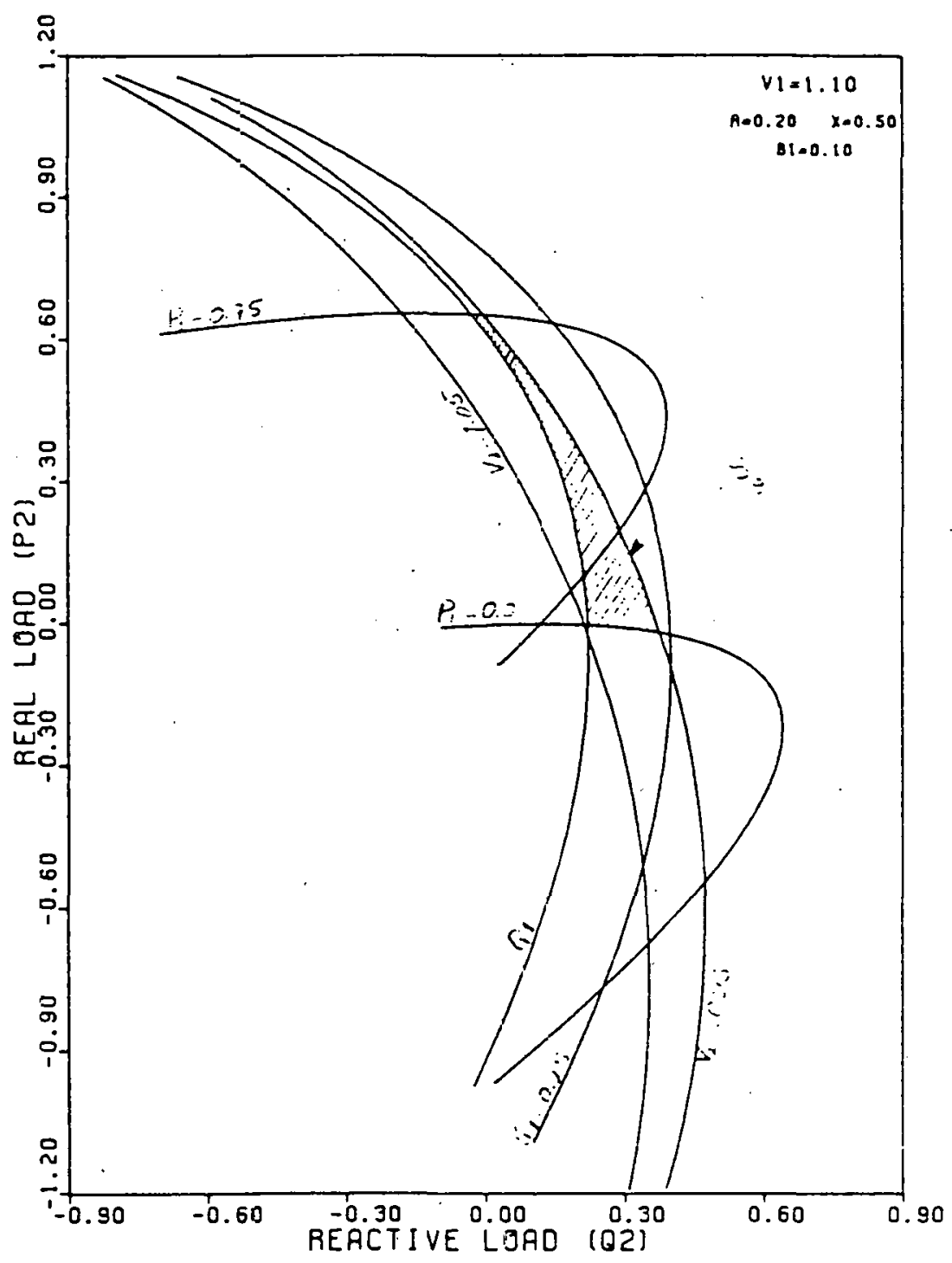

Fig. $(6-3 c)$ 

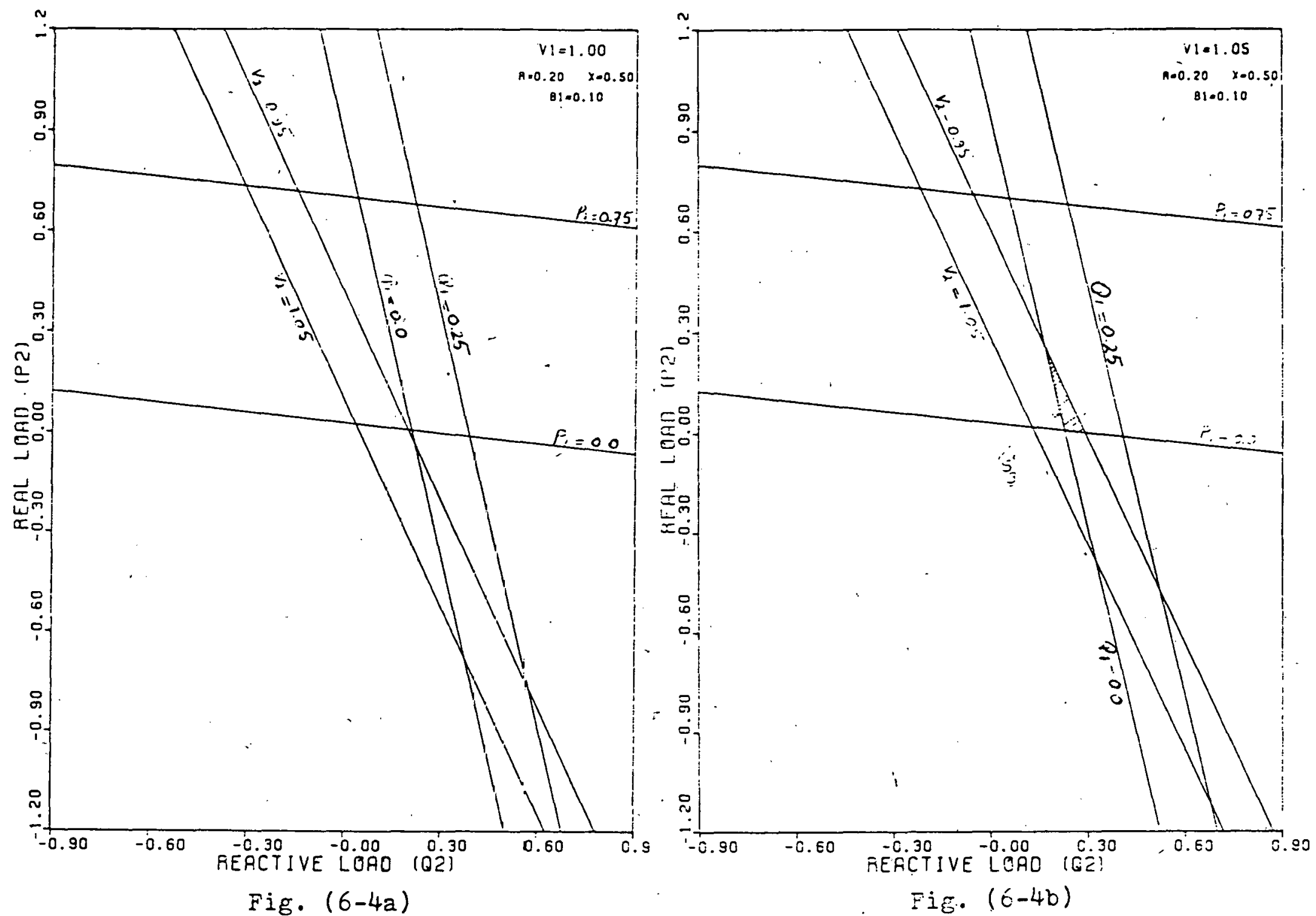

Fig. $(6-4, a, b, c)$ - Three-cross-sections of the linear approximation to $\mathrm{S}_{2}$. 


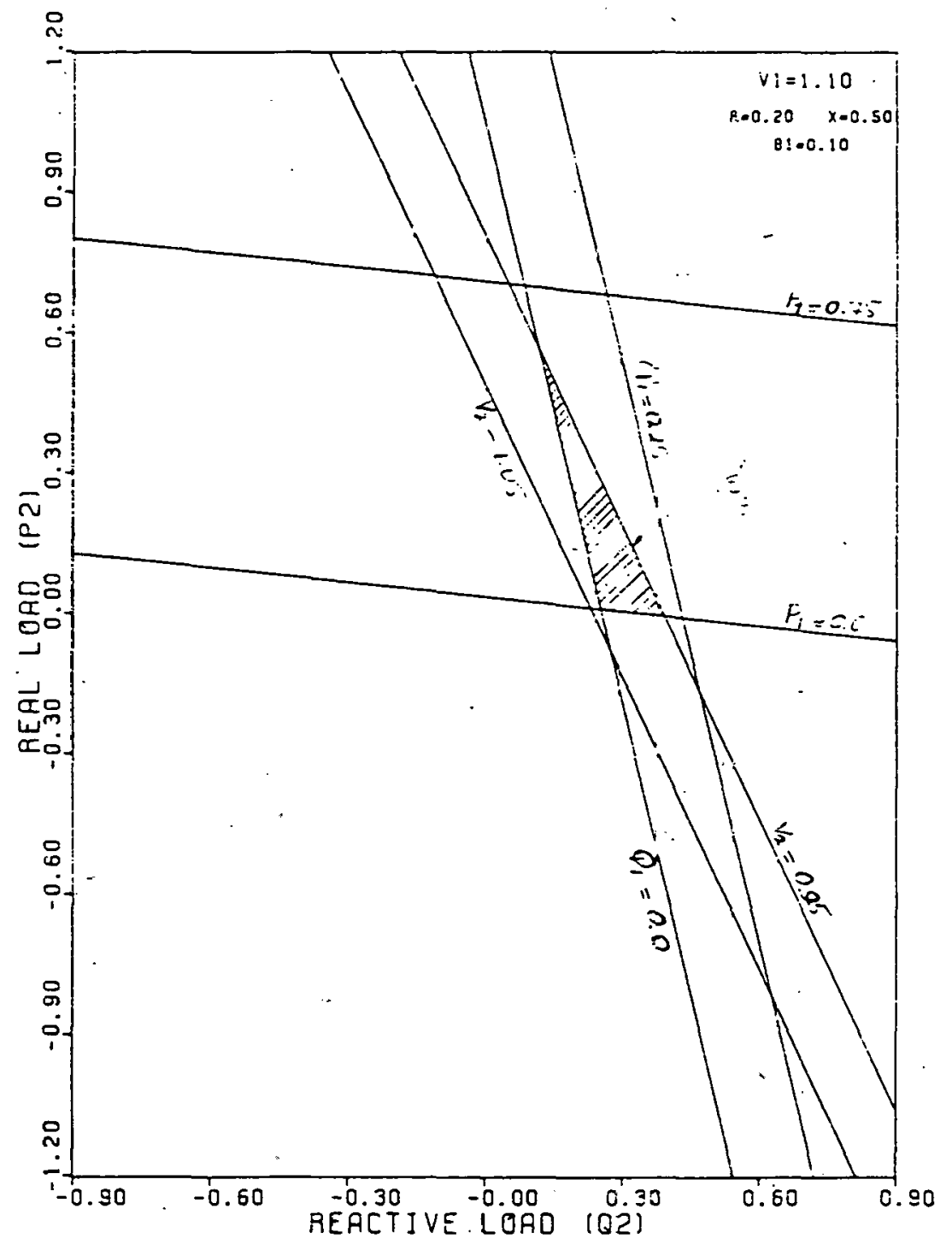

Fig. $(6-4 c)$

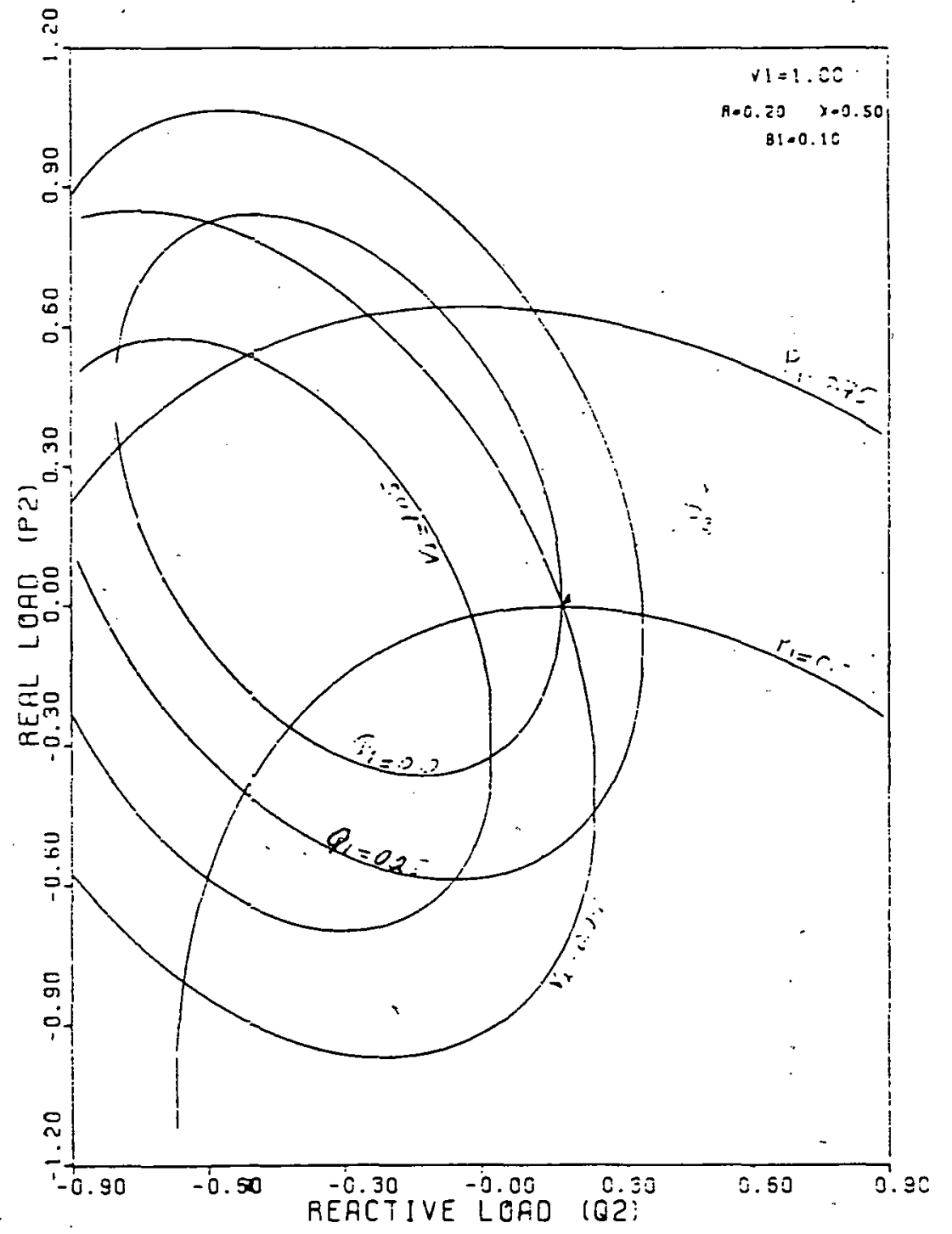

Fig. (6-5a)

Fig. $(t-5, a, b, c)$ - Three cross-sections of the H quadratic approximation to $\mathrm{S}_{z}$. 


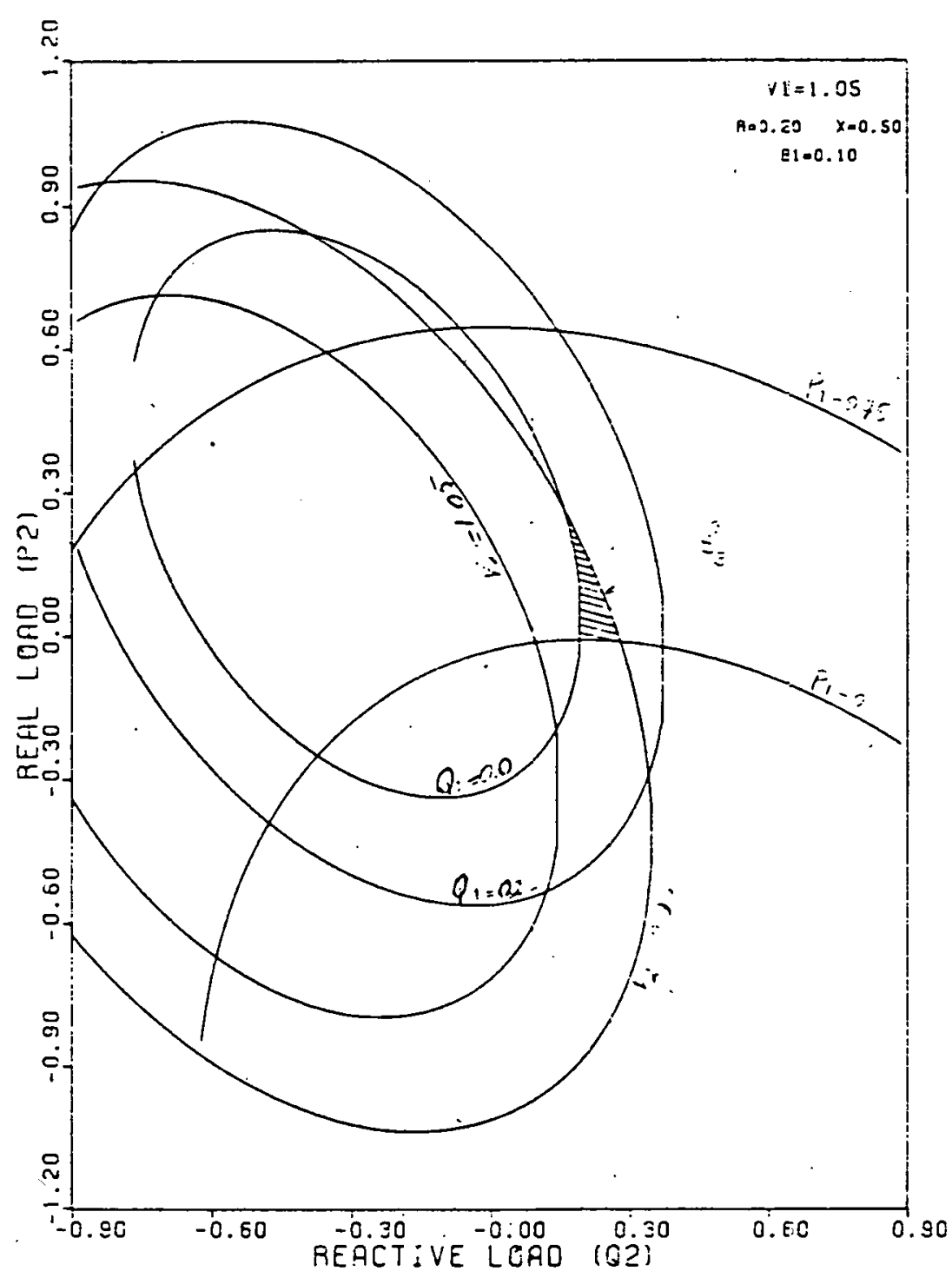

Fig. $(6-5 b)$

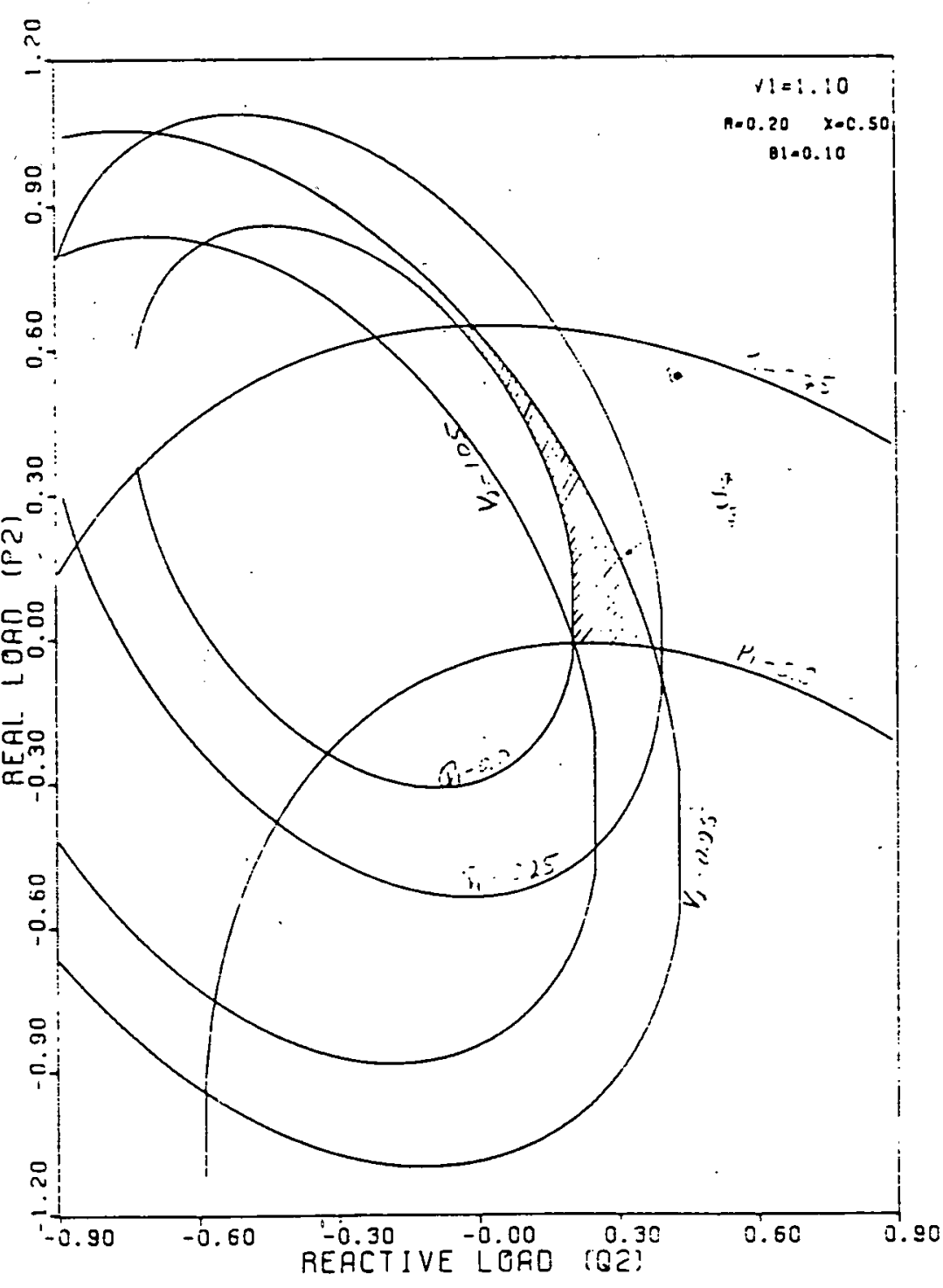

Fig. $(6-5 c)$ 
2) To know which of the hyper-surfaces that form $\mathrm{S}_{z}$ are close to the operating point.

A simple, explicit function would allow him to quickly check any operating point against a sufficient condition. If after a while the check failed, then a knowledge of the hypersurfaces close to the old operating point would help him to choose a sub-set of constraints - from an extremly large set to be monitored. If the new operating point proved to be inside $S_{z}$, then he can generate a new function and repeat the same process all over again.

Consider a sphere surrounding $\underline{z}_{g}$, where $\underline{z}_{g}$ is $\underline{a}$ secure operating point. To enclose the largest possible portion of $S_{z}$, one should look for the sphere which is centred at $\underline{z}_{g}$ ' placed totally inside $S_{z}$, and touching at least at one point of the boundary of $S_{z}$. To describe this sphere only a knowledge of its radius, $r$, is needed, since it can be simply expressed by

$$
\left(\underline{z}-\underline{z}_{g}\right)^{\mathrm{T}}\left(\underline{\mathrm{z}}-\underline{\mathrm{z}}_{\mathrm{g}}\right) \leqslant \mathrm{r}^{2}
$$

To find $r$, one can solve a series of independent problems. Each problem involves finding the radius of a sphere centred at $\underline{z}_{g}$ and touching a hyper-surface, corresponding to a security bound, at one point. Then $r$ is obviously the smallest of all the radii, i.oe.

$$
r=\min \left\{\left(r_{i} i=1, \ldots, i\right)\right\}
$$


where $I$ is the number of constraints and $r_{i}$ is obtained from solving the following problem:

$$
\text { Minimize: } \quad r_{i}^{2}=\left(\underline{z}-\underline{z}_{g}\right)^{T}\left(\underline{z}-\underline{z}_{g}\right)
$$

subject to

$$
Y_{i}=\underline{B}_{i}^{T} \underline{z}+\underline{z}^{T}\left[C_{i}\right] \quad \underline{z}=Y_{i}^{\underline{T}},
$$

here $Y_{i}^{1}$ represents the upper or lower limit on $Y_{i}$. To solve this problem we employ the Lagrange multiplier approach. The Lagrangian function is

$\mathcal{L}=\left(\underline{z}-\underline{z}_{g}\right)^{T}\left(\underline{z}-\underline{z}_{g}\right)+2 \gamma_{i}\left(\underline{\beta}_{i}^{T} \underline{z}+\underline{z}^{T}\left[C_{i}\right] \underline{z}-Y_{i}^{l}\right)$

and the Lagrange multiplier is chosen to be $2 \gamma_{i}$. The radius is given by

$$
r_{i}=\left\|\underline{z}-\underline{z}_{g}\right\|
$$

where $\mathrm{z}$ is a solution to the following system of non-linear equations:

$$
\begin{aligned}
& \left(\frac{\partial \mathcal{L}}{\partial \underline{z}}\right)^{T}=\left(\underline{z}-\underline{z}_{g}\right)+\gamma_{i}\left(\underline{\beta}_{i}+2\left[C_{i}\right] \cdot \underline{z}\right)=\underline{0} \\
& \frac{\partial \mathcal{L}}{\partial \gamma_{j}}=\underline{\beta}_{i}^{T} \underline{z}+\underline{z}^{T}\left[C_{i}\right] \underline{z}-Y_{i}^{I}=0
\end{aligned} .
$$

Equations (12) can be rearranged to read

$$
\left\{[I]+2 \gamma_{i}\left[C_{i}\right]\right\} \underline{z}=\underline{z}_{g}-\gamma_{i-i}^{\beta}
$$


Solving $\mathrm{z}$ from the above equation and inserting it into (13) results in a single equation in terms of $\gamma_{i}$. The resulting equation can be shown to be a polynomial of degree $2(2 N-1)$, indicating that in general $2(2 \mathrm{~N}-1)$ solutions are possible. This poses a possible difficulty when resorting to numerical techniques to solve this set of non-linear equations. A poor initial guess could easily lead to divergence or convergence to an unacceptablesolution. The above set of equations have proven in practice to be extremely sensitive to the initial estimate of the solution; thus the choice of a starting point. is a critical step.

The difficulty in choosing a starting point can be overcome by noting the fact that, as it was mentioned earlier, the hyper-planes

$$
\underline{B}_{i}^{T} \underline{T}=Y_{i}^{1}
$$

are themselves good approximation to the original hypersurfaces. Using equation (15) instead of (14) one can arrive at an explicit solution, i.e.

$$
\begin{aligned}
\gamma_{i} & =\frac{1}{\| \underline{\beta}} \| 2\left(Y_{i}^{l}-\underline{\beta}_{i}^{T} \underline{z}_{g}\right) \\
\underline{z} & =\underline{z}_{g}+\gamma_{i} \underline{\beta}_{i}
\end{aligned}
$$

and

$$
r_{i}=\frac{1}{\left\|\underline{\beta}_{i}\right\|}\left|Y_{i}^{1}-\underline{\beta}_{i}^{T}{ }_{g}\right|
$$

(see fig. (6-4)) 


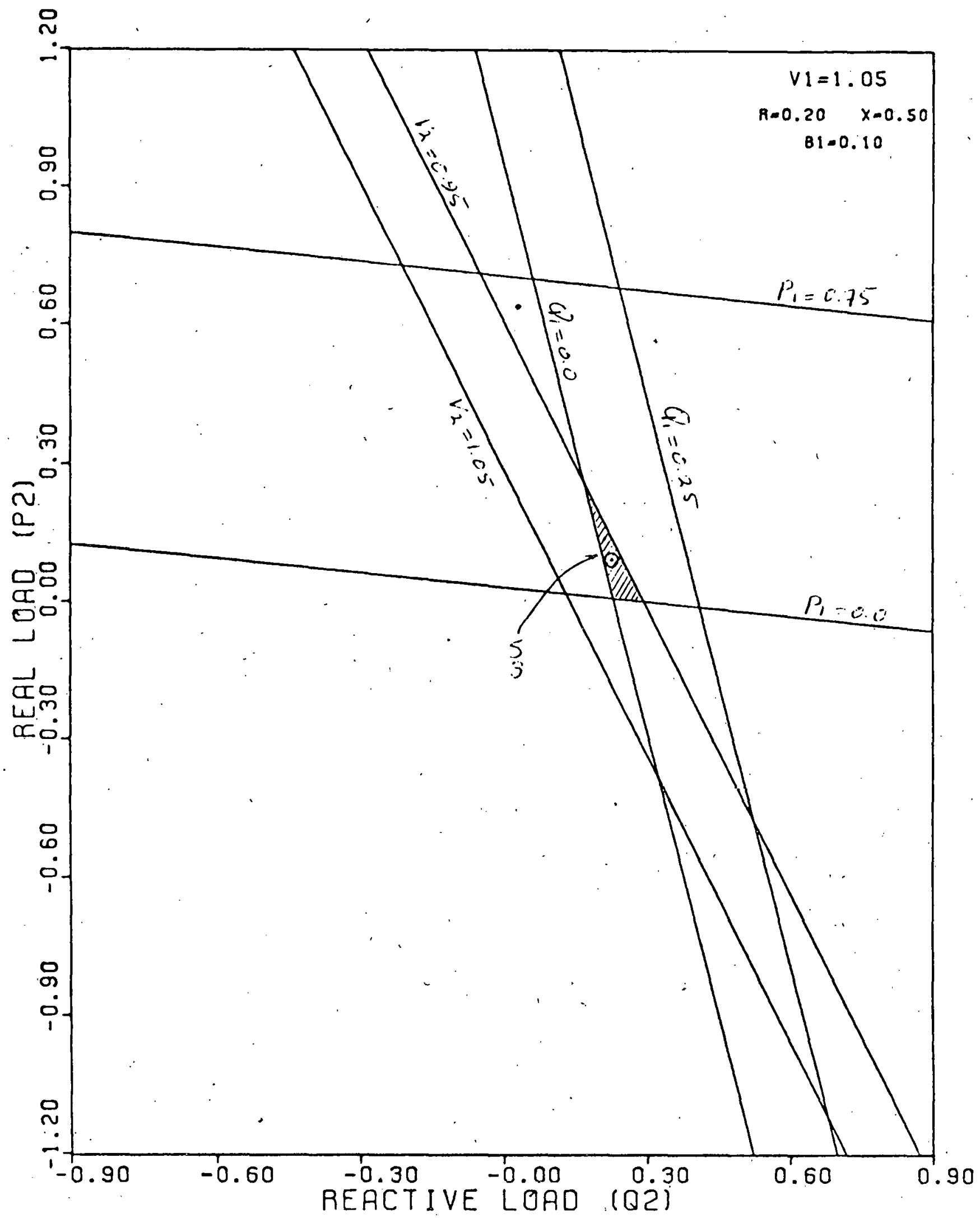

Fig. $(6-6)$ - Fitting a sphere inside the linear secure set of fig. $(6-4)$. 
This solution can be used as an initial estimate to the solution of the original problem. The result will be a fast convergence, as experienced in practice.

The radius $r_{i}$ calculated from (17) is usually very close to that obtained from (11). This suggests that one can solve the problem very quickly by simply ignoring all the quadratic terms in the expansion formulae. This will be a trade off between accuracy and simplicitly if not efficiency. A compromise would be to identify first the hyper-surface which correspends to the smallest radius, using (17), and then solve the original problem for that hyper-surface only.

Listing the calculated $r_{i}$ in an ascending order can serve as a rough indicator of the probable order in which the corresponding constraints could be violated.

To solve the set of non-linear equations in (13) and (14), one can use any standard algorithm developed for this purpose. It is however possible to exploit further the simplicity and efficiency of dealing with linear constraints and devise an easy algorithm on that basis.

Let $\underline{z}_{0}$ represent a starting point to this system of equations then one can approximate equation (13) in each iteration by

$$
\stackrel{\star T}{B_{i}^{T}} \underline{z}=Y_{i}^{l}
$$


where

$$
\stackrel{\star}{\hat{B}}_{i}=\underline{B}_{i}+\left[\mathrm{C}_{i}\right] \underline{z}_{0}
$$

Equations (16) then can be used for $\underline{B}_{i}=\stackrel{\star}{B}_{i}$ to calculate $\gamma_{i}$ and $\underline{z}_{\text {. This }} \underline{\mathbf{z}}$ will serve as a new $\underline{\mathbf{z}}_{0}$ and the iteration continues until two successive $\gamma_{i}$ have almost the same value. A good starting point for this algorithm has proved to be $\underline{z}_{0}=\underline{0}$. It is not suprising, noting that the solutions given in (16) would thus be produced in the first iteration.

The problem of surrounding $\underline{z}_{\mathrm{g}}$ with an ellipsoid can actually be reduced to the problem of surrounding it by a sphere, i.e. the problem just solved. Here instead of equation (8), one has to minimize:

$$
h(\underline{z})=(\underline{z}-\underline{z})^{T}[A]\left(\underline{z}-\underline{z}_{g}\right)
$$

where [A] is a real, symmetric, and positive definite matrix. Its entries are fixed and can be determined experimentally. Since [A] is positive definite, and symmetric, its eigenvalues are all real and positive. This allows one to decompose [A] into

$$
A=[M]^{T} \Lambda^{\frac{1}{2}} \Lambda^{\frac{1}{2}}[M]
$$

where $[M]$ is its modal matrix and $\Lambda$ is the diagonal matrix of eigen-values. Now defining [T] by

$$
[\mathrm{T}]=[\mathrm{M}] \Lambda^{\frac{1}{2}}
$$


one can write $h(\underline{z})$ by,

$$
h(\underline{z})=\left([T] \underline{z}-[T] \underline{z}_{g}\right)^{T}\left([T] \underline{z}-[T] \underline{z}_{g}\right)
$$

This means after transforming all the injection vectors by [T], one essentially ends up with the same problem described by $(8)$ and $(9)$.

\subsection{Filtering out the Redundant Constraints}

The problem just discussed is in fact a crude way of filtering out redundant constraint. To improve over that approach, one can look for the largest possible ellipsoid that can be placed inside $S_{z}$. This is in fact an attempt to express explicitly the largest possible portion of $\mathrm{S}_{\mathrm{z}}$. Mathematically speaking here one is optimizing (8) by not only manipulating $\underline{z}$, but at the same time $\underline{z}_{g}$.

The largest secure sphere touches at least $2 \mathrm{~N}$ sides ( $2 \mathrm{~N}-1$ is the dimension of the injection space) of $\mathrm{S}_{z} \cdot$ We start looking for such spheres. To find a sphere with such a property, we propose a systematic procedure. Let $\underline{z}_{g}$ be any point inside $\mathrm{S}_{\mathrm{z}}$. Using the linear expansion formulae to express the constraints in the injection space, it is easy to calculate the closest distance of $\underline{\mathbf{z}}_{\mathrm{g}}$ to all hyperplanes (formed from setting the constraints at their bounds). The closest distance of $\underline{z}_{g}$ to the hyper-plane " $i$ " is simply given by (17). Suppose the smallest of these correspond to 
the hyper-plane 1 , see fig. $(6-6)$. If we move $\underline{z}_{g}$ away from this hyper-plane, $r_{i}$ will certainly increase while its distance to some other hyper-planes decreases. Let us move $\underline{z}_{g}$ along the line:

$$
\underline{z}=\underline{z}_{g}+\gamma_{1} \underline{\beta}_{1}
$$

by changing $\gamma_{1}$. The distance from any point on this line to the $i-$ th hyper-plane is

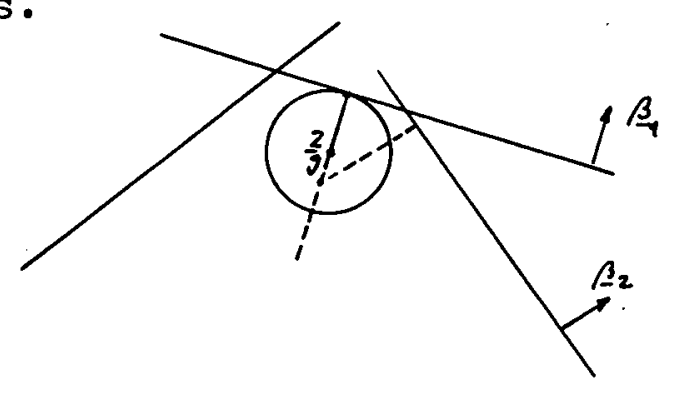

Fig. $(6-6)$

$$
r_{i}=\frac{1}{\left\|\underline{\beta}_{i}\right\|}\left|Y_{i}^{l}-\underline{\beta}_{i}^{T}\left(\underline{z}_{g}+\gamma_{1} \underline{\beta}_{1}\right)\right|=\left|a_{i}+\alpha_{i} \gamma_{1}\right|
$$

where

$$
\begin{aligned}
& \alpha_{i}=\frac{\beta_{i}^{T} \underline{\beta}_{1}}{\left\|\underline{B}_{i}\right\|} \\
& a_{i}=\frac{\underline{\beta}_{i}^{T} \underline{z}_{g}-Y_{i}^{l}}{\left\|\underline{\beta}_{i}\right\|}
\end{aligned}
$$

The adopted strategy is to move $\underline{\mathrm{z}}_{\mathrm{g}}$ along the line $\underline{z}=\underline{z}_{g}+\gamma_{1} \underline{\beta}_{1}$ till its distance from plane 1, i.e. $r_{1}$, equals to its distance to some other plane. In this way we are guaranteed to:

1) remain always inside $S_{z}$.

2) keep the distance of $\underline{z}_{g}$ to other hyper-planes larger or at leat equal to the minimum distance at all times.

The last item actually explains why by continuing this procedure one finally ends up with a desired $\underline{z}_{g^{*}}$ 
To find the first hyper-plane whose distance from a point on the line (23) equals $r_{1}$, one has to solve

$$
\left|a_{1}+\alpha_{1} \gamma_{1}\right|=\left|a_{i}+\alpha_{i} \gamma_{1}\right| \quad i=2, \ldots, L
$$

for $\gamma_{1}$. The smallest $\gamma_{1}, \gamma_{1}^{*}$, is the desired solution. Once the signs of $a_{1}, a_{1}, a_{i}$ and $i$ are known, solving $\gamma_{1}$ from (27) is an easy task. The new $\underline{z}_{g}$ is now:

$$
\underline{z}_{g}^{*}=\underline{z}_{g}+r_{1}^{*} \underline{B}_{1}
$$

Now suppose $\gamma_{1}^{*}$ corresponds to the hyper-plarie 2. Tht next step is then to search over a two dimensional plane the plane of $\left(\gamma_{1}-\gamma_{2}\right)$ - satisfying

$$
\underline{z}=\underline{z}_{g}^{*}+\gamma_{1} \underline{\beta}_{1}+\gamma_{2} \underline{\beta}_{2}
$$

Here one looks for a point on this plane which is not only equi-distant from hyper planes 1 and 2 but also has the same distance from, another hyper-plane. To generalize the problem, one can consider a situation where a point $\underline{z}_{g}$ is, equidistant from $\mathrm{k}$ hyper-planes. In this case, one would like to find a direction along which the distances to these hyperplanes remain equal - but not fixed - and the radius of the sphere car bue increased. Igain one has to start with

$$
\underline{z}=\underline{z}_{g}+\sum_{j=1}^{k} \gamma_{j} \underline{\beta}_{j}
$$

i.e. the search is initially carried out over a k-dimensional plane. The distance of any point $\underline{z}$ belonging to this plane to 
the hyper-plane $i$ is

$$
\begin{aligned}
\dot{r}_{i}=\frac{1}{\left\|\underline{\beta}_{i}\right\|} \mid\left(\underline{\beta}_{i}^{T} \underline{z}_{g}-Y_{i}^{l}\right) & +\sum_{j=1}^{k} \gamma_{j} \underline{\beta}_{i}^{T} \underline{\beta}_{j} \mid \\
& =\left|a_{i}+\varepsilon_{i}\right|
\end{aligned}
$$

where

$$
\varepsilon_{i}=\frac{1}{\left\|\underline{\beta}_{i}\right\|} \underline{B}_{i}^{T}\left(\sum_{j=1}^{k} \gamma_{j} \underline{B}_{j}\right)
$$

To keep $r_{1}=r_{2}=\ldots=r_{k}$, we have to have:

$$
\begin{aligned}
& \delta_{1} \varepsilon_{1}=\delta_{2} \varepsilon_{2} \\
& \vdots \\
& \delta_{k-1} \varepsilon_{k-1}=\delta_{k} \varepsilon_{k}
\end{aligned}
$$

where

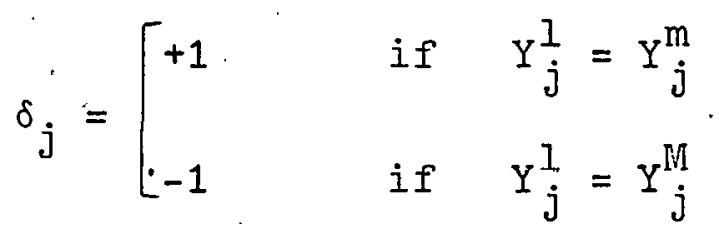

The number of equations in (32) is always $k-1$. This allows one to write the linear system of equations in (32) as

where

$$
[D] \underline{\gamma}=\gamma_{k} \underline{b}
$$

$$
\underline{\gamma}^{T}=\left(\gamma_{1}, \gamma_{2}, \ldots, \gamma_{k-1}\right)
$$

and $[D]$ and $[\underline{b}]$ are merely functions of $\underline{\beta}_{-j} \cdot(j=1, \ldots, k)$. Solving equation (34) for $\left(\underline{\gamma} / \gamma_{k}\right)$ and substituting into (31) it follows 


$$
\varepsilon_{i}=\frac{i}{\left\|\underline{\beta}_{i}\right\|} \underline{\beta}_{i}^{T}\left(\underline{\beta}_{k}+[B][D]^{-1} \underline{b}\right) \gamma_{k}=\alpha_{i} \gamma_{k}
$$

where $[B]$ is a matrix whose columins are $\underline{B}_{j}(j=1, \ldots, k-1)$ and $\alpha_{i}$ denotes the multiplicand of $\gamma_{k}$ in (36).

$$
\text { Replacing } \varepsilon_{i} \text { in (30) with that obtained in (36), one }
$$

ends up with

$$
r_{i}=\left|a_{i}+\alpha_{i} \gamma_{k}\right|
$$

which is essentially equation (24). This means the problem has been reduced once again to a one dimensional search. Thus $\gamma_{\mathrm{k}}^{*}$ can be determined in exactly the same way as $\gamma_{1}^{*}$. This can be continued until the number of equations in (32) exceeds the dimension of the space $\underline{z}$.

The final result is a point $\mathrm{z}_{\mathrm{g}}^{*}$, which with the largest possible secure sphere centred at it, that sphere touches $2 \mathrm{~N}$ sides (hyper-planes) of $\mathrm{S}_{z} \cdot$ Note that this point is not necessarily the point which fits the largest possible sphere inside $S_{Z}$, but it is definitely one of the points which allows one to have a large sphere. In fact if a different $\underline{z}_{g}$ is chosen, most pro-. bably one will end up with a different $\underline{z}_{g}^{*}$ which may or may not improve over the former. Some or all the hyper-planes touching the sphere corresponding to the new $\underline{z}_{g}^{*}$ may be different from the previous ones, thereby allowing another set of active constraints to be identified.

After determining $\underline{z}_{g}^{*}$, one can surround it with an 


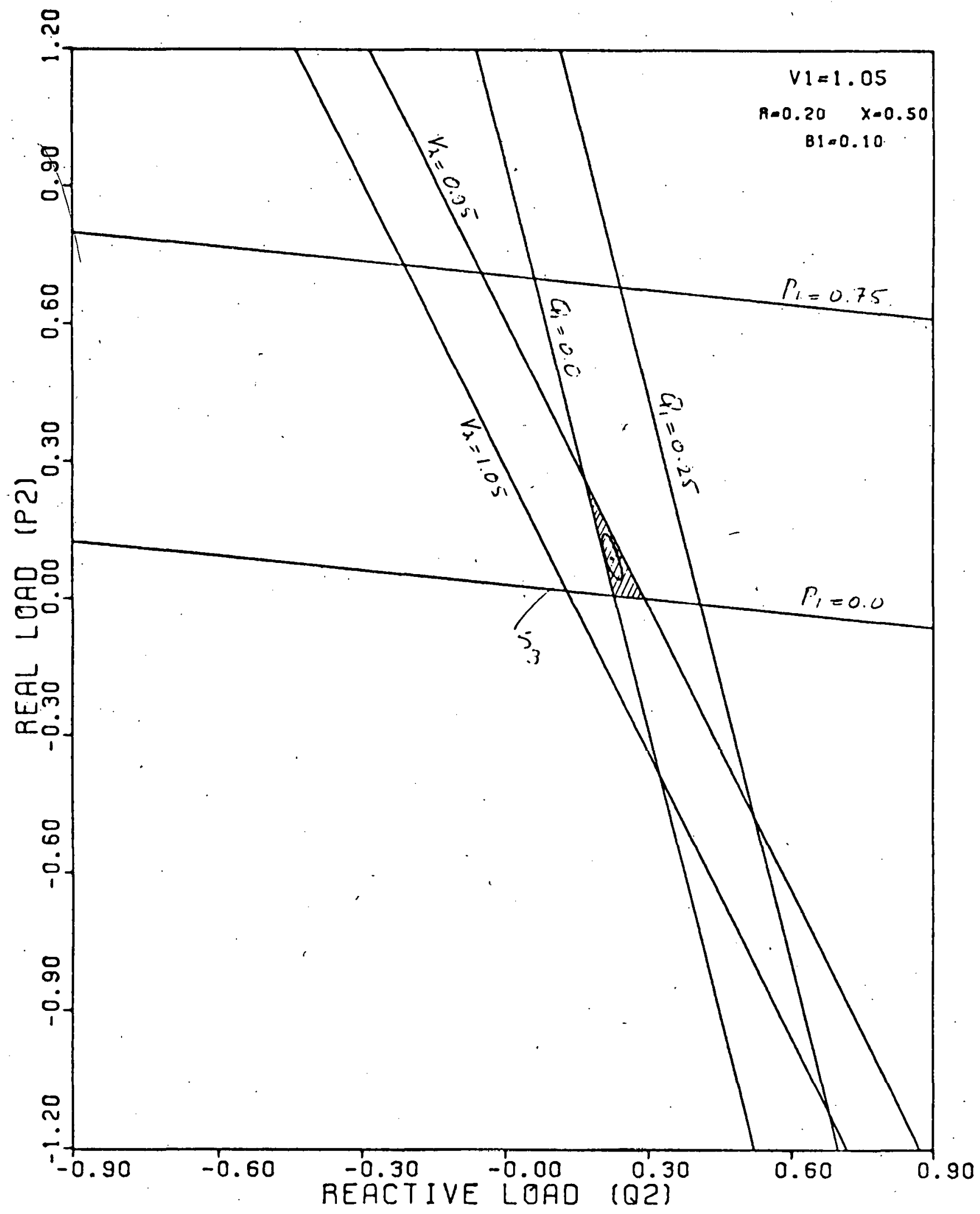

Fig. (6-7)-Fitting an ellipse inside the linear secure set of fig: $(6-4)$. 
ellipsoid to encompass as much of $S_{z}$ as possible - fig. (6-7).

The linear transformation of $\mathrm{S}_{\mathrm{x}}$ into injection space approximates $\mathrm{S}_{2}$ by a linear, convex set. This convexity can be exploited for characterization of larger parts of $\mathrm{S}_{z}$. In the above it was demonstrated that one can start with a small sphere inside the linear set and by following certain stright-forward steps end up with a large sphere. Now consider a line segment joining two points, each belonging to one of the two spheres. Because of the convexity, this line segment lies entirely inside the linear set. If one connects all two such points. together, the result will be a solid conical-shape structure (more precisely, an ice-cream cone!) which also lies intirely inside the set - see fig. (6-8). It is easy to characterize this structure, once the point $\underline{z}_{0}$ and the angle $\theta_{0}$ (shown in the picture) are determined. These two parameters are essential in defining that part of the structure which is not defined by the spheres.

Let us denote the dis-

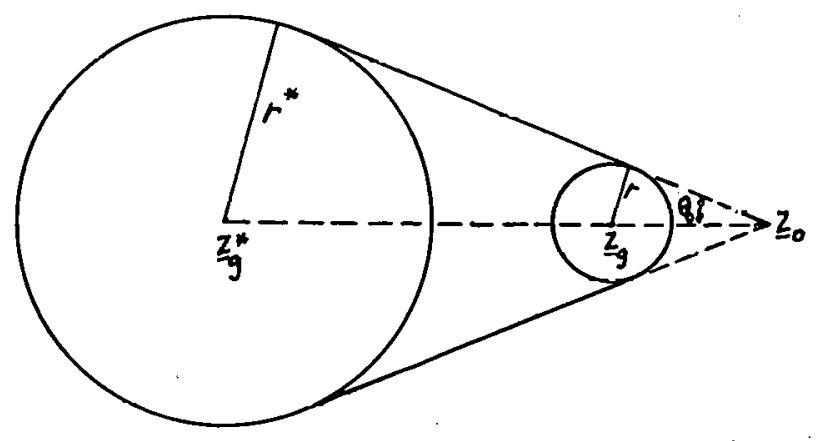
tance between the centres of the spheres by $d, i . e$. Fig. $(6-8)$

$$
d=\left\|\underline{z}_{g}^{*}-\underline{z}_{g}\right\|
$$

Using the elementry geometry, the distance between $\underline{\mathrm{z}}_{\mathrm{g}}$ 
and $\underline{z}_{0}$ is

$$
d_{0}=\left(\frac{r d}{r^{*}-r}\right)
$$

where $r$ and $r^{*}$ are the radii of the small and large spheres respectively. To find $\underline{z}_{0}$, consider the line which passes through points $\underline{z}_{g}^{*}$ and $\underline{z}_{g}$. Any point $\underline{z}$ belonging to this line satisfies

$$
\underline{z}=\left(\frac{t}{d}\right) \underline{z}_{g}+\left(1-\frac{t}{d}\right) \underline{z}_{g}^{*}
$$

where $t$ is the line-parameter. For $t=d+d_{0}$ in $(38), \quad z=z_{0}$. Thus

$$
\underline{z}_{0}=\left(\frac{r^{*}}{r^{*}-r}\right) \underline{z}_{g}-\left(\frac{r}{r^{*}-r}\right) \underline{z}_{g}^{*}
$$

The angle $\theta_{0}$ is simply given by

$$
\theta_{0}=\tan ^{-1}\left(\frac{r^{*}-r}{d}\right)
$$

Now to determine, for example, whether an operating point $\underline{z}$ is inside this structure or not, one can take the following steps:

i) Check whether $\left\|\underline{z}-\underline{z}_{\mathrm{g}}^{*}\right\| \leqslant r^{*}$

ii) Check whether $\left\|\underline{z}-\underline{z}_{g}\right\| \leqslant r$

iii) find $\left\|\underline{z}-\underline{z}_{0}\right\|$ and $\alpha$ where

$$
\alpha=\cos ^{-1}\left[\left(\underline{z}-\underline{z}_{0}\right)^{T} \underline{K} / \| \underline{z}-\underline{z}_{0}^{\| l}\right.
$$

and $\underline{K}$ is a fixed vector given by

$$
\underline{K}=\frac{\underline{z}_{g}^{*}-\underline{z}}{\left\|\underline{z}_{g}^{*}-\underline{g}_{g}\right\|}
$$


iv) Check whether

$$
\alpha \leqslant \theta_{0}
$$

and

$$
d_{0}^{\prime} \leqslant\left\|\underline{z}-\underline{z}_{0}\right\| \leqslant d+d_{0}
$$

These checks are all sufficient conditions and the success of one is sufficient to guarantee that $\underline{z}$ is inside the conical structure and thus it is secure. Note that this structure is of interest provided that

$$
d \geqslant r+r^{*}
$$

i.e. when the two spheres do not over-lap.

\section{5 other Formulations of the Fitting Problem}

Although the transformation formulae, from the theoritical point of view, are very attractive and they open many avenues to research in security related problems, their application in on-line control and security monitoring would suffer a major drawback. For such applications, one needs to have all $\underline{\beta}_{i}$ and $\left[\mathrm{C}_{i}\right] \quad(i=1, \ldots, I)$ stored. Considering the number of constraints present in a large power system, the storage requirement is excessive.

Three approaches, which can totally or partially elimthis drawback, are examined in this sub-section: Here, by formulating the problem in voltage space, one either completely avoids working in the injections space, or delays that to 
the last step. Knowing that the problem of fitting ellipsoids inside $\mathrm{S}_{\mathrm{z}}$ or $\mathrm{S}_{\mathrm{x}}$ can be reduced to fitting spheres, only the latter case is considered.

In the first approach, an exact solution to the fitting. problem is sought. The solution here is approached back-wards, i.e. the expressions involving $z$ are transformed into the voltage space, where an exact, explicit description of all the expressions is possible. Once more, the problem to solve is

$$
\text { Minimize: } \quad\left(\underline{z}-\underline{z}_{g}\right)^{T}\left(\underline{z}-\underline{z}_{g}\right)
$$

subject to

$$
Y_{i}(\underline{x})=Y_{i}^{I}
$$

The load-flow equations, which relate $\underline{z}$ to $\underline{x}$, can be expressed explicitly (see Appendix A-6) by

$$
\underline{z}=[L(\underline{x})] \underline{x}
$$

where $[\mathrm{L}(\underline{\mathrm{x}})]$ is twice the Jacobian of the system. The dependent variables defining the constraints can also be represented by a quadratic form in $x$, i.e.

$$
Y_{i}=\underline{x}^{T}\left[R_{i}\right] \underline{x}
$$

where $\left[R_{i}\right]$ is a highly sparse real symetric matrix. Using these explicit expressions the above problem can be re-stated as

$$
\text { Minimize: } \quad\left([L(\underline{x})] \underline{x}-\underline{z}_{g}\right)^{T}\left([L(\underline{x})] \underline{x}-z_{g}\right)
$$

subject to (43). Here one can minimize (44) over $x$. Again 
using the Lagrange multiplier approach, one ends up with the following set of equations to solve.

$$
\begin{gathered}
\left\{\sum_{k=1}^{2 N-1}\left(\underline{x}^{T}\left[J_{k}\right] \underline{x}-z_{g k}\right)\left[J_{k}\right]+\gamma_{i}\left[R_{i}\right]\right\} \underline{x}=0 \\
\underline{x}^{T}\left[R_{i}\right] \underline{x}=Y_{i}^{1}
\end{gathered}
$$

where $z_{g k}$ are entries of $\underline{z}_{g}$ and $\left[J_{k}\right]$ are counter-parts of $\left[R_{i}\right]$ for nodal injections (see Appendix A-6). It is obvious that a direct attempt to solve the above non-linear problem is formidable. However, it is not difficult to suggest an iterative procedure.

Let $\underline{x}_{0}$ represent a " good " starting point, then one can solve for $\left(\underline{x} / \gamma_{i}\right)$ from

$$
\left\{\sum_{k=1}^{2 N-1}\left(z_{k}\left(\underline{x}_{0}\right)-z_{g k}\right)\left[J_{k}\right]\right\}\left(\underline{x} / \gamma_{i}\right)=-\left[R_{i}\right] \underline{x}_{0}
$$

and $\gamma_{i}$ from

$$
\gamma_{i}= \pm\left\{Y_{i}^{l} /\left(\frac{\underline{x}}{\gamma_{i}}\right)^{T}\left[R_{i}\right]\left(\frac{\underline{x}}{\gamma_{i}}\right)\right\}^{\frac{1}{2}}
$$

Note that in pursuing this algorithm one has to resolve the following problems:

1) What strategy snould one follow in the event that the expression in the bracket, (48), becomes negative.

2) Which sign in (48) is the proper one. 
The second approach which can be considered either as an independent approximate approach or as a preliminary step in solving the exact problem, involves linearization of $\mathrm{z}$ in (41). A change in the injection vector, $\mathrm{z}$, can be approximated by

$$
\Delta \underline{z}=\underline{z}-\underline{z}_{g}=2\left[L\left(\underline{x}_{g}\right)\right] \underline{x}
$$

where $\underline{x}_{g}$ is the solution to (42) for $\underline{z}=\underline{z}_{g}$. This can be used to express (41) in terms of $\underline{x}$ in a simple quadratic form, i.e.

$$
\left(\underline{z}-\underline{z}_{g}\right)^{T}\left(\underline{z}-\underline{z}_{g}\right)=\left(\underline{x}-\underline{x}_{g}\right)^{T}[D]\left(\underline{x}-\underline{x}_{g}\right)
$$

where

$$
[D]=4\left[L\left(\underline{x}_{g}\right)\right]^{T}\left[L\left(\underline{x}_{g}\right)\right]
$$

is a real symetric and positive definite matrix (with the assumpition that $\mathrm{x}_{\mathrm{g}}$ exist, $\left[\mathrm{L}\left(\underline{\mathrm{x}}_{g}\right)\right]$ cannot be singular). Minimizing (50) subject to (46), requires solving

$$
\begin{gathered}
\left\{[D]+\gamma_{i}\left[R_{i}\right]\right\} \underline{x}=[D] \underline{x}_{g} \\
\underline{x}^{T}\left[R_{i}\right] \underline{x}=Y_{i}^{l}
\end{gathered}
$$

which is relatively easy. The solution, when inserted in (44), provides the radius of a sphere centred at $z_{g}$ in the injection.space. It can also serve as a very good starting point to the exact problem. The accuracy of this approach is far better than that obtained from the linear transforma- 
tion, but at most equal to that which uses quadratic transformation.

In the third approach, first one needs to fit an ellipsoid inside $S_{X}$. This problem can be solved readily. It involves solving a series of problems of the following type:

$$
\text { Minimize: } \quad h(\underline{x})=\left(\underline{x}-\underline{x}_{g}\right)^{T}[A]\left(\underline{x}-\underline{x}_{g}\right)
$$

subject to (43). To solve this problem, one has to obtain a solution to

$$
\begin{array}{r}
\left\{[I]+\gamma_{i}\left[\hat{R}_{\dot{i}}\right]\right\} \underline{\hat{x}}=\hat{x}_{g} \\
\qquad \underline{\hat{x}}\left[\hat{R}_{\dot{i}}\right] \underline{\hat{x}}=Y_{i}^{I}
\end{array}
$$

where

$$
\begin{aligned}
\hat{\underline{x}} & =[\mathrm{T}] \underline{x} \\
{\left[\hat{R}_{\dot{1}}\right] } & =[\mathrm{T}]\left[R_{\dot{1}}\right][T]^{T}
\end{aligned}
$$

and $[T]$ is an ortho-normal matrix given by (21). The equations in (54) and (55) are again relatively easy to solve and do not involve too much intricate computations. The final result will be an ellipsoid given by

$$
\left(\underline{x}-\underline{x}_{g}\right)^{T}[A]\left(\underline{x}-\underline{x}_{g}\right) \leqslant h_{\min }
$$

where $h_{\min }$ is the smallest of all $h(\underline{x})$ found in each sub-problem. This is an exact solution and the ellipsoid given in (56) does not contain any inccoure point. Now one can use the transforma- 
tion formulae to transform the quadratic expression in (56) from the voltage space into the injection space. The result for a quadratic transformation will be

$$
\underline{z}^{T}[E] \quad \underline{z} \leqslant d
$$

where

$$
\begin{gathered}
{[E]=\frac{1}{4}\left(\left[L\left(\underline{x}_{g}\right)\right]^{-1}\right)^{T}\{[A]-[J(\underline{\alpha})]\}\left[L\left(\underline{x}_{g}\right)\right]^{-1}} \\
\underline{\alpha}=\underline{x}_{g}^{T}[A]\left[I\left(\underline{x}_{g}\right)\right]^{-1}
\end{gathered}
$$

and

$$
d=h_{\min }-\underline{x}_{g}^{T}[A] \underline{x}_{g}
$$

The accuracy can be increased practically to any degree by using higher-order terms. The calculations and storage requirements here are limited to that of $\underline{\alpha}$ and [E], which are quite modest.

\subsection{Present and Projected Works}

In connection with the subject of this section, we are presently engaged in studying the following topics:

(a) Fast and reliable algorithms for finding the largest ellipsoid and the largest sphere in $\mathrm{S}_{\mathrm{z}}$.

(b) Methods of bringing an insecure point into the secure region via the shortest possible path, with and 
without considering the equality constraints (i.e. power balance equation).

(c) Inproved numerical methods for fast computation of the solutions to equations (44) and (45).

(d) Characterization of the vulnerability set.

(e) Development of a general algorithm for secureeconomic operation of power-systems, based on additional features of the linear expansion formulae.

(f), Employment of load forcasting data for selecting parts of $S_{z}$ to be characterized. 


\section{APPENDIX A-6}

(see the accompanying paper) 


\title{
APPROXIMATION FORMULAE, FOR DEPENDENT LOAD FLOW VARIABLES
}

\author{
F.D. Goliana, IEEE Member, and M. Banakar \\ Department of Electrical Engineering \\ MCGill University \\ Montreal, Quebec, Canada.
}

\section{ABSTRACT}

General formulae are systematically derived which explicitly relate any dependent load flow variable to the independent injections. Dependent variables include line current magnitudes, reactive generations, load bus voltage magnitudes, slack generation, and real losses. The independent injections are defined by $P$ and $V$ at the $P V$ buses, $P$ and $Q$ at the load buses and $\checkmark$ in the slack bus. The formulae derived are 1 inear, quadratic or higher order in the injections. A theoretical and numerical analysis of these formulae is carried out providing quantitative data on their range of validity. This indicates a level of accuracy, particularly for second order formulae, sufficiently high for many applications.

\section{INTRODUCTION}

Power systems in steady state are characterized by a set of non-linear algebraic equation of the form

$$
\underline{\mathbf{z}}=\underline{\mathbf{F}}(\underline{x})
$$

known as the load-flow equations [1]. . The vector of injections $z$ contains the rea at the load buses, $z_{L}$, as well as the controllable Injections, $\underline{u}$ (voltage levels and real injections) at the generation buses. Thus

$$
\underline{z}=\left[\begin{array}{l}
\underline{u} \\
\underline{z} \\
\underline{L}
\end{array}\right]
$$

while $\underline{x}$ represents the complex bus voltages.

To operate a power system under economical and operational constraints, it is desirable to monitor andor control certain dependent variables (e.g. line. flows, load voltage levels, reactive generations, losses). If y represents a typical dependent variable, then

$$
y=g(\underline{x})
$$

where $g$ is an explicit function of the state $x$.

Theorctically the existence of a solution to (1) is equivalent to the existence of a function, $\underline{F}^{-1}$ mapping $\underline{z}$ back into the $\underline{x}$ space, namely

$$
\underline{x}=\underline{F}^{-1}(\underline{z})
$$

Disregarding the existence and uniqueness problem associated with this transformation, any variable $y$ can be expressed in terms of $\underline{z}$.

$$
y=g\left(\underline{F}^{-1}(\underline{z})\right)
$$

This function cannot in general be computed as a closed form relation in $z$ because of the nonlinear nature of $g$ and $\underline{F}$.

It should be noted that today there exist many efficient numerical schemes which compute $F^{-1}(z)$ for a given $z$ (load flow), and which manipulate $\bar{u}$ in such a way that some function of $\underline{F}^{-1}\left(\underline{z}_{L}, \underline{u}\right)$ is minimized (optimal load flow). Explicit functions approximating $F^{-1}(z)$ have nevertheless played an important role in many aspects of system security, economic dispatch, and system planning. Some authors have preferred to approach the problem of establishing explicit relations between $y$ and $z$ by linearizing the load-flow equations. Examples of this include the concept of distribution factors [2], security regions based on the DC load flow approximation $[3,4]$, and various applications in optimal load flow $[5,6,7]$. Most of these cases have resorted to linear approximation formulae because of their suitability to analysis via linear programming methods. There exists one notable example of a quadratic approximation formulae, namely the B-coefficients loss formulae $(8,9)$ still used in economil dispatcir.

In this paper we systematically treat the subject of approximation formulae for general load flow variables.

The main results of this study are:

1. A systematic definition and derivation of explicit linear, quadratic, and higher order formulae between an arbitrary dependent variable, $y$, and the injections of a general load-fluw problen, z.

2. Analytical treatment of the approximation errors. Derivation of special properties useful. in the numerical implementation of these formulae.

3. A comparison with other special approximation formulae such as the B-ccefficients loss formulae, and the DC load flow loss and line power flow approximation formulae.

4. A numerical error analysis of the derived formulae, using a random simulation approach on sample systems.

5. Discussion of profected and potential uses of these approximation formulae in security monltoring and enhancement. 
BACKGROUND

Load Flow Equations in guadratlc Form

The material in this section is based on formulations developed in 110,11$]$, where the special quadratic form structure existing between voltage and power is exploited to establish certain limitations and properties inherent to power systems. Here for the sake of completeness, some of the basic formulations are summarized.

by

Denoting the complex bus voltage, $v_{i}$, at bus $i$

$$
v_{1}=e_{i}+j f_{i}
$$

we define a $2 \mathrm{~N}$ vector

$$
\underline{x}=\left[\begin{array}{l}
\underline{e} \\
\underline{f}
\end{array}\right]
$$

with $e^{T}=\left(e_{1}, e_{2}, \ldots . e_{k}\right)$

$$
\text { and } \quad \underline{\mathrm{E}}^{\mathrm{T}}=\left(\mathrm{E}_{1}, \mathrm{f}_{2}, \ldots \ldots, \mathrm{f}_{\mathrm{N}}\right)
$$

where $\mathrm{N}$ is the number of buses and $T$ stands for the transpose of a vector.

Now let $z_{k}$ represent a component of $z$. (i.e: $\ddot{e}_{k}, q_{k}$ ór $\left.v_{k}^{2}\right)$. It follows that

$$
z_{k}=\underline{x}^{T} \underline{J}_{k} \underline{x}
$$

The $2 \mathrm{~N} \times 2 \mathrm{~N}$ constant real symmetric matrices $J$ are defined uniquely by the type of injections and the network structire. If $\underline{G}=\left\{g_{i j}\right\}$ and $\underline{B}=\left\{b_{i j}\right\}$ represent, the real and imaginary part of the system admittance matrix respectively, then for a real power injection:

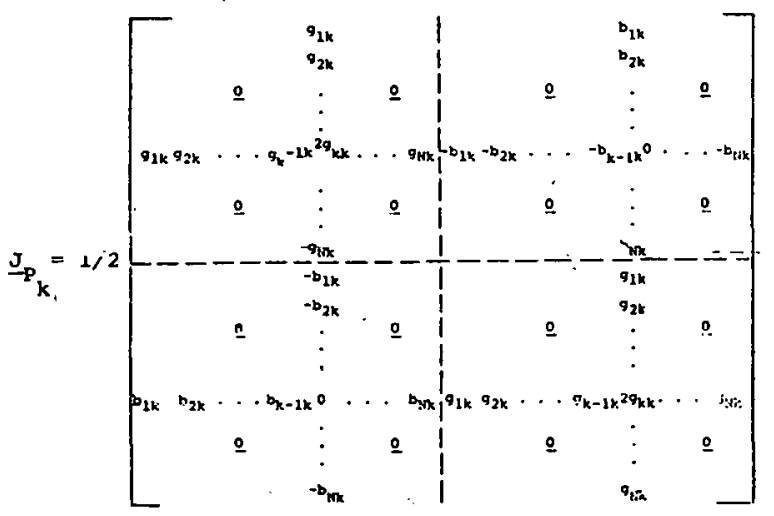

(7)

From (7) it is clear that. $\mathrm{J}_{\mathrm{k}}$ is highly

oparse; the only possible non-zero elelunts 11 e in two columns and two rows (the kth and $k+N(h)$. Similarly for a reactive power injection one has.

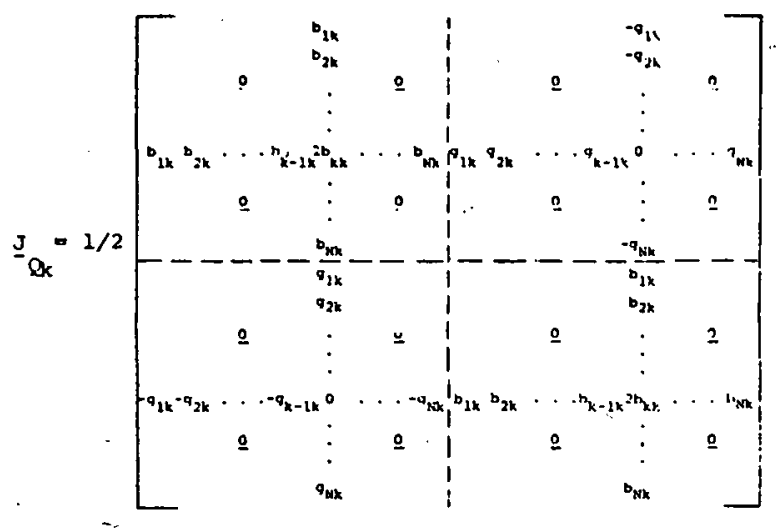

(8)

When voltage injections are considered $\left(z_{k}=v_{k}{ }^{2}\right.$. are $2 \mathrm{~N} \times 2 \mathrm{~N}$ real matrices where all the entries are zero except $(k, k)$ and $(k+N, k+N)$, which are equal to one. The above expressions can be readily verified from the rectangular version of the load flow equations, i.e.

$$
\begin{aligned}
& F_{j}=\sum_{i=1}^{N} e_{j}\left(g_{j i} e_{i}-b_{j i} f_{i}\right)+f_{j}\left(g_{j i} f_{i}+b_{j i} e_{i}\right) \\
& Q_{j}=\sum_{i=1}^{N} f_{j}\left(g_{j i} e_{i}-b_{j i} f_{i}\right)-e_{j}\left(g_{j i} f_{i}+b_{j i} e_{i}\right)
\end{aligned}
$$

and

$$
v_{j}^{2}=e_{j}^{2}+f_{j}^{2}
$$

At the slack bus both $e_{j}$ and $f_{j}$ are specified quantities and one can set $f_{j}$ equal to zero without loss of generality. Then the dimension of $x$ reduces to $2 \mathrm{~N}-1$, while the matrices $J_{z k}$ are correspondingly simplified to $2 \mathrm{~N}-1$ by $2 \mathrm{~N}-1$.

Equation (6) can be used to express $\underline{z}$ in the form of equation (1), that is,

$$
\underline{z}=[L \underline{(x)}] \underline{x}
$$

where we define the $2 \mathrm{~N}-1$ by $2 \mathrm{~N}-1$ matrix $\underline{L}(\underline{x})$ as,

$$
\underline{\underline{L}} \underline{\underline{x}}=\left[\begin{array}{c}
\underline{x}^{\mathrm{T}}\left[J_{z_{2}}\right] \\
\underline{x}^{T}\left[J_{z_{2}}\right] \\
\vdots \\
\vdots \\
\underline{x}^{T}\left[J_{\left.z_{2 N-1}\right]}\right]
\end{array}\right]
$$

The following properties of the above formulation are essential to the proceeding derivations:

$$
\text { 1. For any constant } 2 \mathrm{~N}-1 \text { vector } \underline{\alpha}
$$$$
\underline{\alpha^{T}} \underline{\underline{z}}=\sum_{i=1}^{2 N-1} a_{1} z_{1}=\underline{x}^{T} \underline{J}(\underline{\alpha}) \quad \underline{x}
$$

whore we have defined

$$
J(a)=\sum_{1=1}^{2 N-1} \alpha_{1}^{J} z_{1}
$$


Note that we can equally write

$$
\underline{a}^{T} \underline{z}=\underline{a}^{T} \underline{L}(\underline{x}) \underline{x}
$$

Comparing equations (11) and (12), It follows that,

$$
\underline{x}^{T} \underline{J}(\underline{a})=\underline{q}^{T} \underline{\underline{L}}(\underline{x})
$$

Note that the matrix $J(x)$ is symmetric and refains a sparsity similar to that of the Jacobian matrix.

2. An important observation is that $L(x)$ is equal to one-half the Jacobian matrix of the load-flow equations. This can be readily verified by partial differentiation of $\underline{z}$ with respect to $\underline{x}$ in $(9)$.

3. To perform $\frac{\partial}{\partial \underline{x}}\{\underline{L}(\underline{x})\}$, a complicated notation is needed to define the resulting three-dimensional matrix. It is, however, possible to bypass this by using the fact that for any constant $2 \mathrm{~N}-1$ vector $\underline{a}$.

$$
\begin{aligned}
\underline{\alpha}^{T} \frac{\partial}{\partial \underline{x}}\{\underline{L}(\underline{x})\} & =\frac{\partial}{\partial \underline{x}}\left\{\underline{\alpha}^{T} \underline{L}(\underline{x})\right\} \\
& =\frac{\partial}{\partial \underline{x}}\left\{\underline{x}^{T} \underline{J}(\underline{a})\right\}=\underline{J}(\underline{a})
\end{aligned}
$$

APPROXIMATION FORMULAE FOR DEPENDENT NETWORK VARIABLES

The formulation of the load-flow problem described in the previous section permits a systematic derivation of explicit approximation formulae for any dependent network variable, $y$, in terms of the injections, $\underline{z}$. Dependent variables such as power line flows, reactive power injections at PV buses, voltage magnitudes squared at $\mathrm{PQ}$ buses, all can be represented by a quadratic form in $x$, i.e.

$$
y=y(\underline{x})=\underline{x}^{T} \underline{R} \underline{y}
$$

where $\underline{R}_{y}$ is a highly sparse real symmetric matrix corresponding to $y$. It should be noted that any linear combination of dependent and/or independent variables can also be described by a quadratic form in $x$. In this way important dependent variables such as the real system losses ur the arorage network valtage squared can be expressed through (15).

Explicit and, as the results indicate, accurate approximation formuale can be systematically derived based on a Taylor series expansion of $y$ in terms of $\underline{z}$, that is

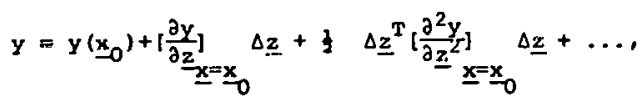

where $\Delta \underline{z} \triangleq \underline{z}-\underline{z}_{0}$ and

$$
\underline{z}_{0}=\underline{L}\left(\underline{x}_{0}\right) \underline{x}_{0}
$$

while $y(\underline{x})$ is defined by (15).

Due to the vectorial nature of the differentiations involved, it is obvious that after the third-term one is faced with a storage problem caused by the increasing dimensionality of the partial derivative tensors produced. Fortunately, as will be shown, the storage requirements of the formulae derived on the basis of this expansion need not be greater than those of the inverse Jacobian in Factorized form. Furthermore, expansions of first or second-order seem to be sufficiently accurate for mäny applicaticins.
Various approximation formulae can be derived on the basis of (16). These are summarized below up to a third-order formula where firgt-order implies a relation linear in $z$, second-order quadratic in $z$, thirdorder cubic in $\underline{z}$, and so on. Their derivation is detalled in Appendix $A$.

Linear Approximation Formula'

Here $Y$ is approximated by the first two terms in (16), which are denoted by $Y_{1}$. Then from Appendix $A$,

$$
y_{1}=\underline{B}_{y}^{T} \underline{z}
$$

where

$$
\underline{B}_{y} \triangleq\left[\underline{L}^{T}\left(\underline{x}_{0}\right)\right]^{-1} \underline{R}_{y} \underline{x}_{0}
$$

Note that because of equation (22) the constant term in the linear expansion drops out.

\section{Error Analysis of the Linear Approximation Formula}

The error associated with the linear approximation to $y$, i.e., $y_{1}$, is denoted by $\varepsilon_{1}$. Thus

$$
y=\underline{B}^{T} \underline{z}+\varepsilon_{1}
$$

where the subscript $y$ has been dropped from $\underline{B}$ and $\underline{R}$ and is understood. The error therefore given by

$$
\begin{aligned}
\varepsilon_{1} & =y-\underline{B}^{T} \underline{z} \\
& =\underline{x}^{T}\{\underline{R}-\underline{J}(\underline{E})\} \underline{x} \underline{\Delta} \underline{x}^{T} \underline{E} \underline{x}
\end{aligned}
$$

where we made use of computation (11). It is easy to see that at $\underline{x}_{0}$ the error $\varepsilon_{1}$ is zero. This is so since

$$
\begin{aligned}
\underline{J}(\underline{B}) \underline{x}_{0} & =\underline{L}^{T}\left(\underline{x}_{0}\right) \underline{B} \\
& =\left[\underline{L}^{T}\left(\underline{x}_{0}\right)\right]\left[\underline{L}^{T}\left(\underline{x}_{0}\right)^{-1}\right] \underline{R} \underline{x}_{0}=\underline{R}_{0} \underline{x}_{0}
\end{aligned}
$$

It follows therefore that $\varepsilon_{1}$ is not only a quadratic function of $\underline{x}$ but also of $\Delta_{x} \underline{x} \underline{\underline{x}}-\underline{x}_{0}$, i.e:,

$$
\begin{aligned}
\varepsilon_{1} & =\Delta \underline{x}^{T}\{\underline{R}-\underline{J}(\underline{B})\} \Delta \underline{x} \\
& =\Delta \underline{x} \underline{E} \Delta \underline{x}
\end{aligned}
$$

Various properties of quadratic forms can therefore be used to analyze the nature of $\varepsilon_{1}$ in order to better understand tne valldity of the linear afproximation formula. Prelininary results for example indicate that for some variables (those that are always non-negative), such as the vultage magnitude squared, magnitude squared of a line current or the real losses, the linear approximation error is always positive or zero when the expansion point is near the flat-voltage profile (condition for zero transmission losses). This implies that the error matrix $\mathrm{E}$ is positive semi-definite. Althougli no rigorous proof of this result in a general form is available, the argument being studied is that the space of feasible $y$ and $z$ for the above mentioned variables is convex near the flat voltage point. In this case the plane $y=\underline{E}^{T} \underline{z}$, which is tangent to the space of feasible $y$ and $z$ at $x_{0}$, must be a support hyper-plane on or below

In suoh cases, we then have for all feasible 2 the condition that $\varepsilon_{1} \geqslant 0$ or,

$$
y \geqslant \underline{B}^{T} \underline{z}=Y_{1}
$$

This condition has been found to be satisfied in extensive experimental runs of systems of up to eleven nodes for variables representing the magnitude of a 
network quantity. It must be polnted out that for real losses and line current magnitudes, (24) is trivially satisfled when $x_{0}$ is the flat voltage profile since for this expansion point $\underline{0}$ and $R \geq 0$. In this case the best lincar approximation of these quantities is zero.

Since the normal operating point of power systems is near the flat-voltage profile, one possible way of exploiting (24) is to generate a large number of B's (i.e. lower bounds on $y$ ) based on expansion points near the flat voltage point. A sufficiently large number of these linear formula may suffice to approxImate a variable $y$ over a wide enough range of $z$ to accurately monitor or control its value.

A more quantitative relation on the error $\varepsilon_{1}$ can be derived from the aninimum $\left(\lambda_{m}\right)$ and maximum $\left(\lambda_{M}\right)$ eigenvaiues of $\underline{E}$.' It can be showr. that

$$
\lambda_{m} h^{2}(\Delta \underline{z}) \leqslant \varepsilon_{1} \leqslant \lambda_{M} h^{2}(\Delta \underline{z})
$$

where

$$
h(\Delta \underline{z}) \Delta \frac{1}{2 c_{2}}\left[-c_{1}+\left(c_{1}^{2}+4 c_{2}\|\Delta \underline{z}\|\right)^{\frac{2}{2}}\right]
$$

The constants $c_{1}$ and $c_{2}$ depend on the network parameters only.

Ao will be seen in the resuits section, the use of one linear approximation formula per variable $y$ is prone to large errors. One way of avoiding this is by having various linear inequalities per variable, as explained above. Another way to make these formula more precise is to go to higher order expansions or to rely on a numerioal load flow scheme.

\section{Quadratic Approximation Formulae}

Few explicit quadratic approximation formulae appear in the literature, probably because of the difficulties associated with the calculation of the second derivative of $y$ with respect to $z$. This section shows how an explicit second-order or quadratic approximation formula can be systematically derived for any dependent variable as a function of the arbitrary injection vector, $\underline{z}$.

Let $y$ be approximated by the first three terms of the Taylor expansion of $y$ in (16), denoted by $Y_{2}$, Then from Appendix $A$,

$$
y_{2}=\underline{\beta}_{y}^{T} \underline{z}+\underline{z}^{T} C_{y} \underline{z}
$$

where $\underline{B}_{y}$ is as before given by (18) while the real symmetric matrix $\underline{c}_{y}$ takes the foril,

$$
S_{y}=\left\{\left[\underline{L}^{T}\right]^{-1}\left\{\underline{R}_{y}-\underline{J}\left(\underline{B}_{Y}\right)\right\} \underline{L}^{-1}\right.
$$

where the abbreviated notation

$$
\underline{\underline{\Delta}} \underline{\underline{L}}\left(\underline{x}_{0}\right)
$$

is used. Once more equation (22) is used to eliminate the constant terms in the second order expansion.

The accuracy of the quadratic approximation formulae, as indicated by the results, is fairly high and for expansion points $x_{0}$ correspnniting to a noirial loading of the system one such equation per varlable $y$ suffices in many applications.
Error Analysis of Quadratic Approximation Formula

If $\varepsilon_{2}$ denotes the error associated with the quadratic approximation to $y_{1}$ i.e. $Y_{2}$, then

$$
\begin{aligned}
\varepsilon_{2}=y-y_{2} & =\underline{x}^{T} \underline{R}-\left(\underline{\beta}^{T} \underline{z}+\underline{z}^{T} \underline{c} \underline{z}\right) \\
& =\left(x^{T} \underline{R} \underline{x}-\underline{\beta}^{T} \underline{z}\right)-\underline{z}^{T} \underline{z} .
\end{aligned}
$$

Exploiting the properties summarized in squ. (21), (22), and (23), and also using the definitions of $(28)$ one can write

$$
\varepsilon_{2}=\varepsilon_{1}-\Delta \underline{x}_{1}^{T} \underline{E} \Delta \underline{x}_{2}
$$

where

$$
\Delta \underline{x}_{-1}=\frac{1}{2}\left(\hat{x}_{1}-\underline{x}_{0}\right)
$$

and

$$
\underline{\hat{x}}_{1}=\underline{L}^{-1} \underline{z}
$$

It will be show in the following soctions that if $x_{0}$ represents the starting point to a NewtonRalphson (NR) load-flow algorithm, then $\Delta x_{\text {l }}$ is the correction added to $x_{0}$ in the next iteration.

Thus from (31), one can conclude that $y_{2}$ is improved over $y$ in a manner comparable but not equal to that achievable in one iteration of the NR algorithm.

By expressing $e_{1}$ in terms of $E, E_{2}$ will be the difference between two terms, i.e.

$$
\varepsilon_{2}=\Delta \underline{x}^{T} \underline{E} \Delta \underline{x}-\Delta x_{1}^{T} \underline{E} \cdot \underline{\underline{x}} i
$$

For those variables for which $E$ is positive semi-definite, both terms in (33) are non-negative, so that $\varepsilon$ can have positive or negative values depending on the value of $x$. It is therefore difficult to make general analytic statements about the error of the second ordex approximation, except that it is comparable to that after one NR load flow iteration.

Relation Between Approximation Formulae and NR Load Flow Alcorithms

Quadratic approximation formulae can be derived from a NR algorithm as follows:

The first iteration of a constant Jacobian NR load flow scheme gives

$$
\underline{x}-\underline{x}_{0} \simeq 1 L^{-1}\left(\underline{z}-z_{0}\right)
$$

or

$$
\underline{x}=1 \underline{L}^{-1}\left(\underline{z}+\underline{z}_{0}\right) \triangleq \underline{x}_{1}^{N R}
$$

which when substituted into (15) ylelds a quadratic formula in $z$.

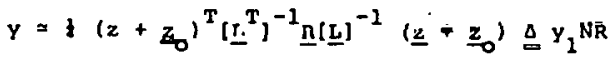

It is easy to see that in general the second order Taylor approximation. $Y_{2}$, differs frum $Y_{1}^{\text {NR }}$ as given in $(36)$, althouyh both are quadratic in $z$. However for certain variables such as losses and ine flows the two formulae are identical if $x_{0}$ is the flat voltage profile. 
It is clear from the NR algorithm that if more 1terations are carried out, any desired accuracy can be achieved. It is interesting to carry out one more iteration of the NR scheme. Then

$$
\underline{x}-\underline{x}_{1}^{N R}=\frac{1}{2} \underline{L}^{-1}\left(\underline{z}-\underline{L}\left(\underline{x}_{1}^{N R}\right) \underline{x}_{1}^{N R}\right)
$$

where $x_{1}^{N R}$ is defined by (35). Substitution of the above approximation of $\underline{x}$ into (15) yields $y_{2}^{N R}$ which is a fourth order expression of $y$ in $z$. Further NR steps would therefore yield expansions of order $8,16,32$, and so on.

The Taylor expansion as a load flow scheme is not competitive with the NR method because of the effort involved in calculating higher order expansions when high accuracy is desired. However, as a means of obtaining linear or quadratic approximation formulae, for applications where high accuracy is not crucial, the Taylor expansion has some advantages. For example, the linear expansion cannot be obtained from a load flow iteration since the order of these jumps from 0 to 2 to 4 and so on. Also, numerical results indicate that in general a second oxder Taylor approximation is more accurate than the second order formula obtained from one NR-iteration.

\section{Third and Higher order Taylor Expansion Formulae}

For completeness the derivation of these formulae is included in Appendix $A$.

\section{Numerical Considerations}

If the linear approximation of a variable is desired, one can bypass the need for evaluating $\mathrm{L}^{-1}$ by solving a system of linear equations. Since $\bar{y}_{1}$ can also be written in terms of $\underline{\underline{x}}_{1}$ then.

$$
y_{1}=\underline{B}^{T} \underline{z}=\underline{x}_{0} \underline{T}_{R}\left[L^{T}\right]^{-1} \underline{z}=\underline{x}_{0}{ }^{T} \underline{R}_{\underline{x}} \underline{\hat{x}}_{1}
$$

The system of equations to be solved can be either

$$
\underline{L}^{T} \underline{B}=\underline{R} \underline{x}
$$

For $\underline{\beta} ;$ or

$$
\underline{\mathbf{L}} \underline{\hat{x}}=\underline{z}
$$

for $\underline{\hat{x}}_{1}$. The first system of equations has the feature of being inciependent from $z$. Note that $\underline{R} \underline{x}_{0}$ can be very efficiently computed as it reprosents a very sparse vector. The matrix $\underline{R}$ need not be explicitly stored, it is simply a convenient notation. In any case the main storage requirements are those of the factorized version of $\mathrm{I}^{-1}$ as this matrix reappears in the computation of $y_{1}$ or $\underline{S}$ for any variable $y$. In the case of structural changes in the network such as those due to a line outage, efficient schemes based on the matrix inversion lemma $[12]$ exist for modifying $L^{-1}$ without having to refactorize it.

For quadratic approximations of a variable, zgain the explicit calculation of $C$ is in most applications unnecessary. Computing and storing $\underline{L}^{-1}$ in factorized form is basically all that is needed to apply (27). The matrix $J(B)$ need not be computed or stored explicitly, nor, as mentioned above, is it necessary to compute $B$. To verify this statement follow the following procedures for calculating the gecond term of (27):

$$
\begin{aligned}
& \text { 1) compute } \underline{\hat{x}}_{1}-\underline{\underline{L}}^{-1}\left(\underline{x}_{0}\right) \underline{z} \\
& \text { 11) compute } \left.\underline{z}\left(\underline{\hat{x}}_{1}\right)=\quad \underline{\underline{\underline{\hat{x}}}}\right) \underline{\hat{x}}_{1} \\
& \text { 111) compute } \underline{\hat{x}}_{2}=\underline{L}^{-1}\left(\underline{x}_{0}\right) \underline{z}\left(\underline{x}_{1}\right)
\end{aligned}
$$

iv) evaluate the term defined by $R, 1 . e$.

$$
\underline{\hat{x}}_{1}^{T} \underline{\hat{x}}_{1}=y\left(\underline{\hat{x}}_{1}\right)
$$

v) The term defined by $\underline{J}(\underline{B})$ is given by,

$$
\left.t \underline{\hat{x}}_{1}{ }^{T} \underline{J}(\underline{B}) \underline{\hat{x}}_{1}=t \underline{B}^{T} \underline{z}^{(\underline{\hat{z}}} \underline{\underline{1}}_{1}\right)=1 \underline{x}_{0}^{T} \underline{R}_{\underline{x}_{2}}
$$

Note therefore that the generalized quadratic formula (27) can be used to approximate any dependent variable, $y$, with some minor additional operations after the computation of $\underline{\hat{x}}_{1}$ and $\underline{\hat{x}}_{2}$ in (40) and (41) respectively. The effort needed to evaluate $y_{2}$ is therefore comparable to two iterations of a constant gradient Newton-Raphson algorithm. The effort involved in a second order Taylor expansion is therefore greater than its counterpart, (36), obtained from a NR algorithm, however its accuracy is in general better. In some applications it may be desirable, to exploit the explicit quadratic structure of the approximation formulae, in which case the Taylor expansion would be advantageous because of the higher precision. For high accuracy, and when no explicit relation between $y$ and $z$ is needed, a load flow is superior.

The explicit computation of $\mathrm{C}$ is in most instances unnecessary and undesirable because of the effort and storage involved. One exception could be in the derivation of loss formulae where the controllable variables, $\underline{u}$, form a small subset of $\underline{z}$. In that case one could derive from (27), under the assumption that $z_{t}$ is constant (see equation (2)), a loss formula of the form

$$
y=a_{u}+\underline{B}_{u}^{T} \underline{u}+\underline{u}^{T} \underline{C}_{u} \underline{u}
$$

where the dimensions of $\underline{B}_{u}$ and $\underline{c}_{u}$ are relatively low.

\section{Other Arplicatinns of Approximation Formulae}

In steady state security munitoring and control the concept of security regions [3] provide new techniques for rapidly correcting vulnerable or emergency situations. The use of linear approximation formulae together with LP schemes has had some success $[3,4]$, in this respect, however the innacsuracy of the linear relations in represcnting certain variables limits this approach suluewhat. The high accuracy of quadratic formulae, as well as their well understood analytic properties, opens up new avenues of researsh in the topic of security regions. Thus, if the variable $y_{i}$ is restricted to lie between $y_{i}^{m}$ and $y_{1}$ because of steady state security restrictions, then

$$
y_{i}^{M} \geqslant Y_{i}=\underline{B}_{i}^{T} \underline{z}+\underline{z}^{T} \underline{C}_{i} z \geqslant, y_{i}^{m}
$$

for $1=1,2, \ldots, k$ where $K$ is the number of restricted dependent variables in the system. Equations (43) define therefore the security regions ( $S$ ) in the space of $z$ characterized by $2 \mathrm{~K}$ quadratic equations in $\underline{z}$. The properties of these equations are presently being investigated to identify redurdant constraints and to obtain simple representations of $s_{z}$ useful in security control. and enhancement. It should be noted that these potential applications do not require the explicit computation or storage of $\underline{B}_{1}$ and $\underline{C}_{1}$, these matrices beling treated only symbolically in thls manner. 
Various potential applications of these approximation formulac exist in the arca of network refuction where lumped equivalents of the effect of the external system can be systematically and accurately represented by linear or quadratic formulao of the tie-line flows.

\section{RESULTS}

\section{A Two Bus System}

A two bus system is examined here primarily because its mathematical model, as opposed to larger systems, involves only a few variables. This enables us to show some of the results graphically, thus providing a better insight into the nature of the expansion formulae.

The system here simply consists of a generation bus and a load bus connected via a line of impedance $z=0.1+j 0.4$ P.U. Figs. (1) through (3) show the equi-8 error contours in the $P_{2} Q_{2}$ plane for the second order Taylor expansions of the real and reactive power generation as well as the voltage squared at the load bus (bus 2). The expansion point corresponds to a load of 1.5 - j1.0 P.U. for a generation bus voltage of 1.0 P.U. The following points in relation to these graphs are of importance:

1. A lärge area around the expansion point can be identified as a very low exror region $(<18)$.

2- The $\pm \infty$ error contour present in Fig. (2) corresponds to the zero reactive generation surface and does not actually mean that its expansion formula is under or over

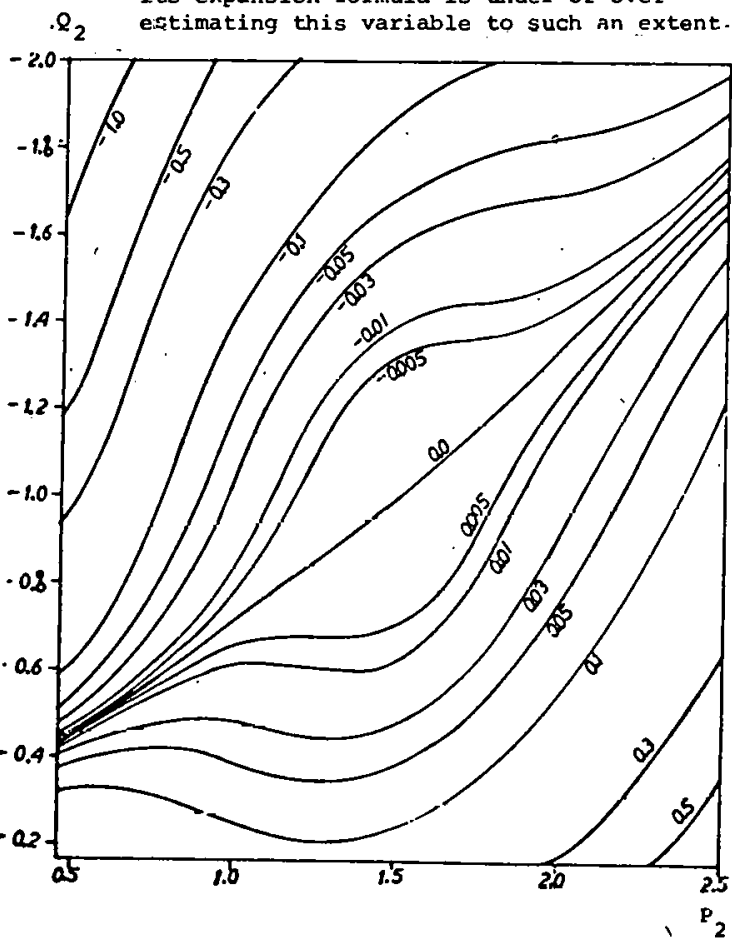

FIG.1 : Equi-8 Error Contoura for Quadrat1c Approximation of the Real Power Generation.

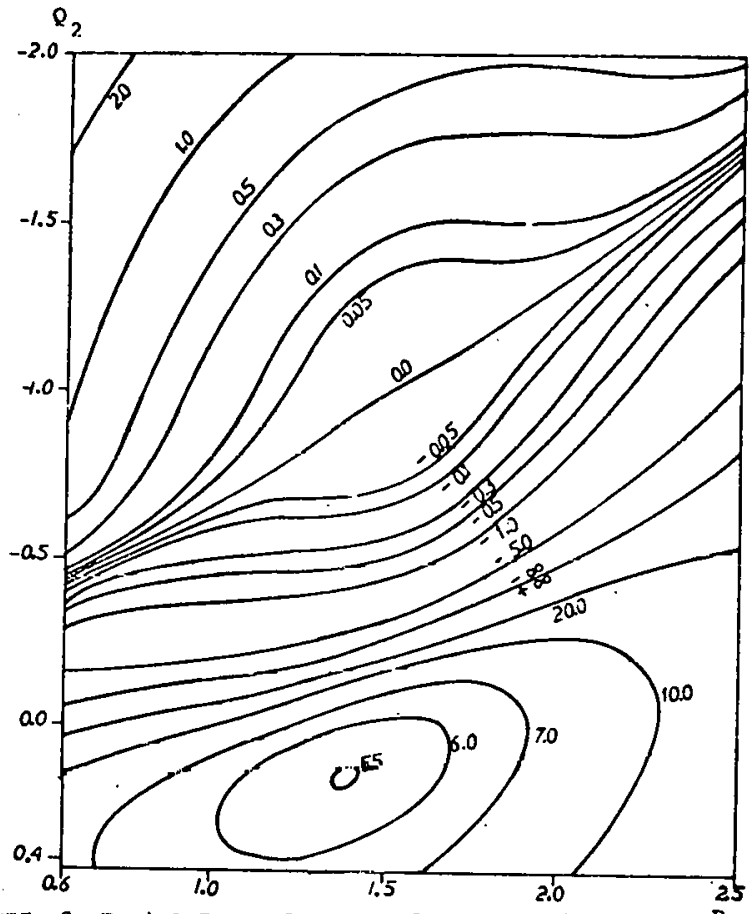

FIG. 2: Equi-8 Error Contours for Quadratic Approx- $\mathrm{P}_{2}$ Imation of the Reactive Power Generation.

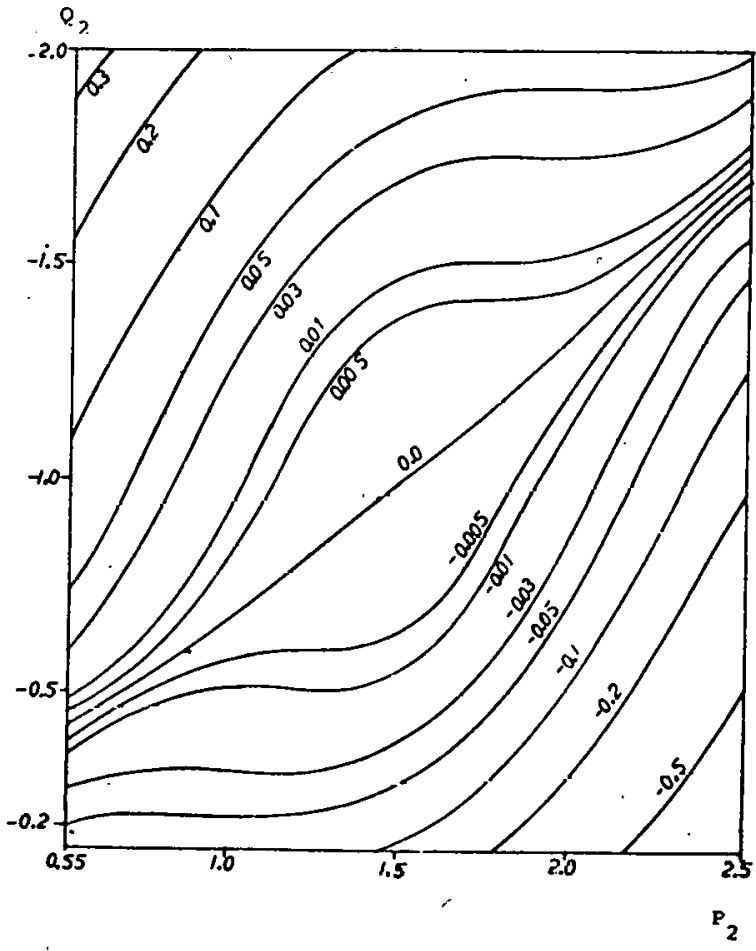

FIG. 3: Equi-8 Error Contours for, Quadratic Approximation of the Voltage squared at the Load Bus. 
vatar pomen centantion ar glack ous
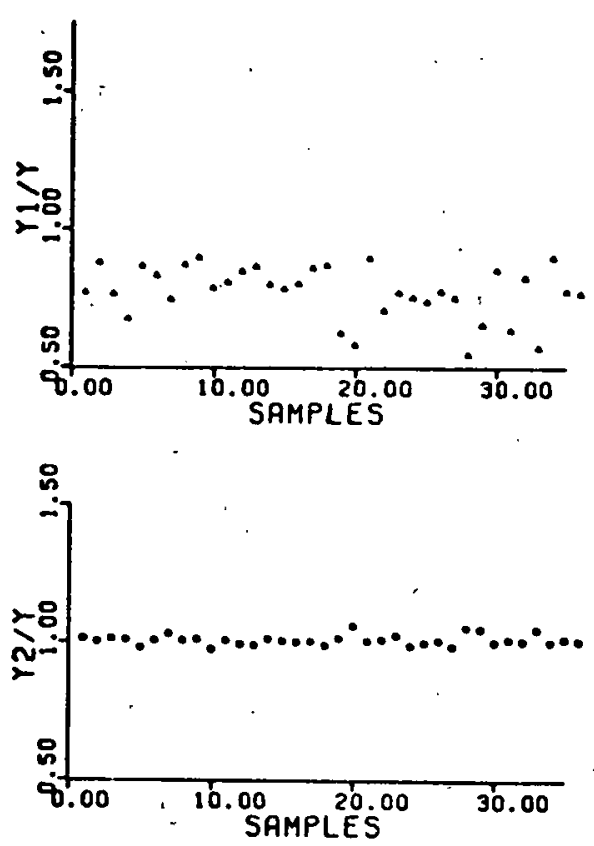

FIG. 4: Typical Error Spread of Linear $\left(y_{1}\right)$ and Quadratic $(y$, Approximation to Real Power Injection at slack Bus for Random operating Points.

T-AEACTIVE POWEN at a GENEAATIOM OUS
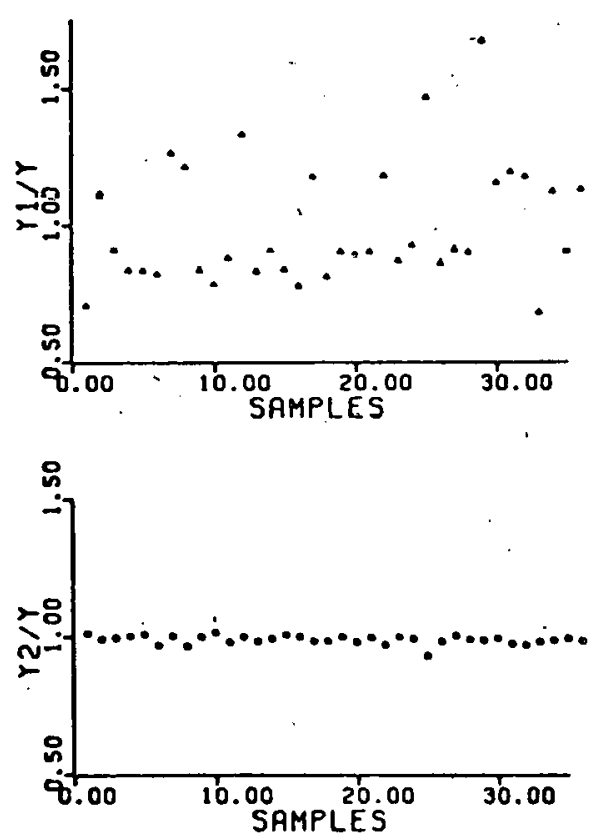

FIG. 5: Typical Error Spread of tinear $(y)$ and Quadratic $\left(y_{2}\right)$ Approximation to Reactive Power Injection at a Generation Bus for kandom Operating roints.
Jovourace gouanco ar a tano ous
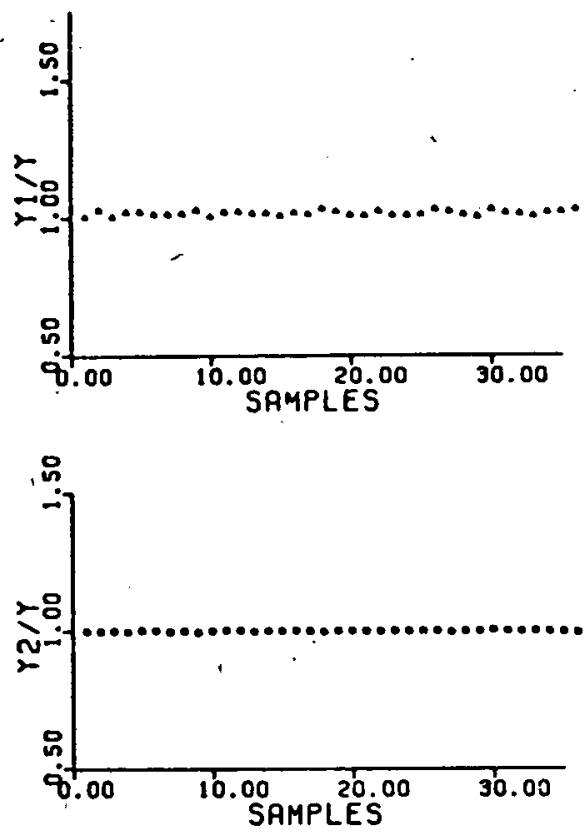

FIG. 6: Typical Error Spread of Linear (y) and Quadratic $\left(y_{2}\right)$ Approximation to vôltage Squared at a Load Bus for Random Operating Points.

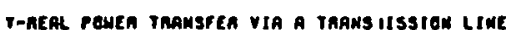
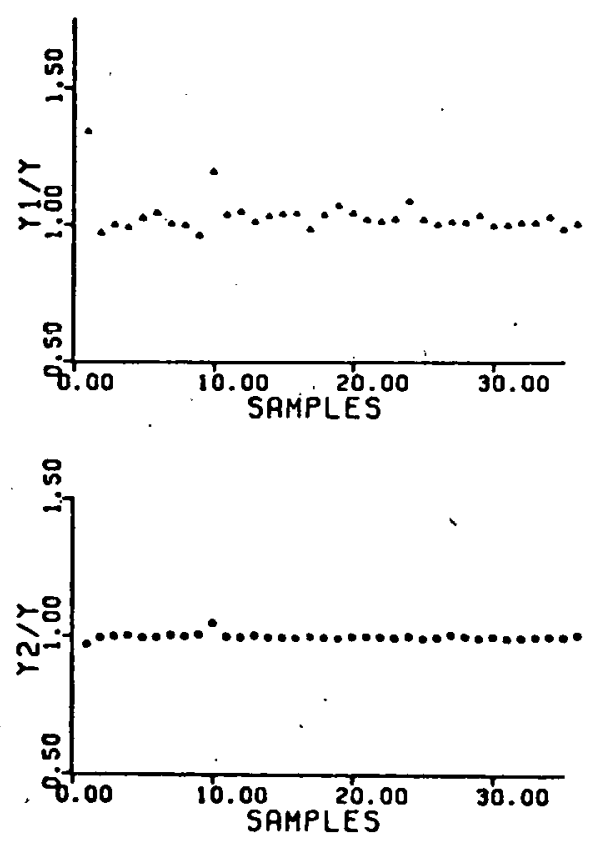

EIG. 7: Typical Error Spread of Linear ( $\left.y_{1}\right)$ and Quadratic $\left(y_{2}\right)$ Approximation to Real Line Flows for Random Operating Points. 


\section{An Elght Rus System}

The elght bus system of [13] is chosen to study the exrors involved in approximating unknown injection variables and power flows. The error analysis here is based on a random simulation approach. Random numbers are generated within a hyper-box in the space of voltages. The sides of the hyper-box are $0.2 \mathrm{p}$.U. Iong and the expansion point, $x_{0}$, is at its'center.

These varlations of $\underline{x}$ around $x_{0}$ generate a wide

spectrum of operating points covering and going beyond their normal expected range. The generated numbers are substituted into the load-flow equations to obtain generations, loads, voltage levels, and power transfers. Those numbers which do not correspond to realistic load flows (1.e. positive real püwer generations, negative real loads) are discarded. After forming the injection vector $z$ fron these data, the expansion formulae are used to calculate the value of the unspecified variables in the system. The risults are then compared with their calculated exact value. A fow typical results for some of the unspecified variables, $y_{1}$, are shown in Figs. (4) through (7). Note that $y_{1}$ and $y_{2}$ are respectively the linear and quadratic approximations. Table 1 below sumarizes these errors

\begin{tabular}{|l|l|l|l|l|}
\cline { 2 - 5 } \multicolumn{1}{c|}{} & $\mathrm{v}_{\text {load }}^{2}$ & $\mathrm{P}_{\text {slack }}$ & $\mathrm{Q}_{\text {slack }}$ & $\mathrm{P}_{\text {line }}$ \\
\hline$\left|\frac{\varepsilon_{2}}{y}\right| \times 100$ & 2 & 20 & 25 & 8 \\
\hline
\end{tabular}

Table 1 Average errors for some typical dependent variables

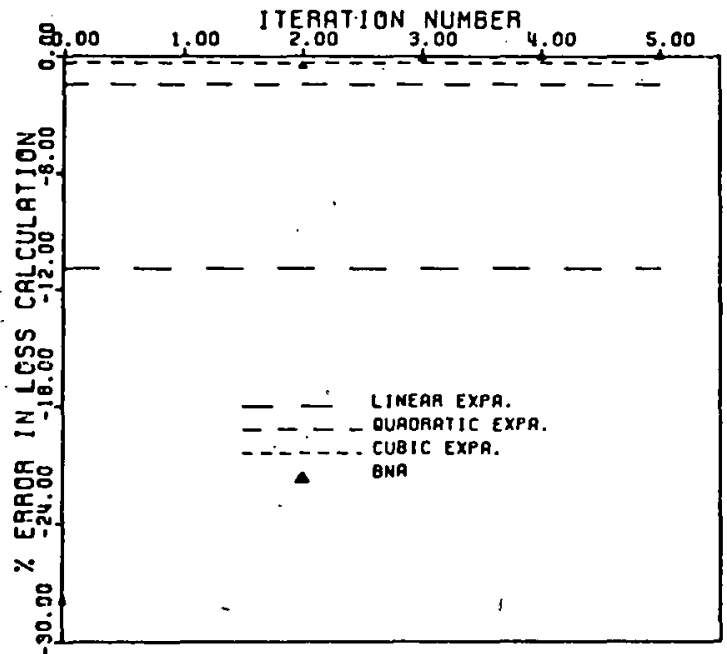

FIG. 8: TYpical Accuracies of the Linear, Quadratic, and Cubic Taylor Expansions of a Dependent Variable (losses) Relative to the N.R. Load Flow Scheme (BNA).
In Fig. (8) the relative accuracy of the expansion formula in calculating total loss in the system is compared with that of the constant gradient Newton Algorithm (BNA). This result, although given for a particular variable (losses) and load distribution, is typical for other variables in the system. Thus supporting our previous statements that for high accuracy the Newton algorithm is superior when a numerical result is the main objective.

\section{An Eleven Bus System}

To compare our expansion formulae with other expansion formulae presently in use, the eleven bus system of [8] is employed. During the last three de-? cades much investigation has been devoted to developing techniques or improving tlie existing techniques in calculating B-coefficients. Thus one can consider the B-coefficient loss formula, as the most sophisticated second order approximation formula. The B-coefficients for the eleven bus system are givon in the above reterence for a loss formula of the form
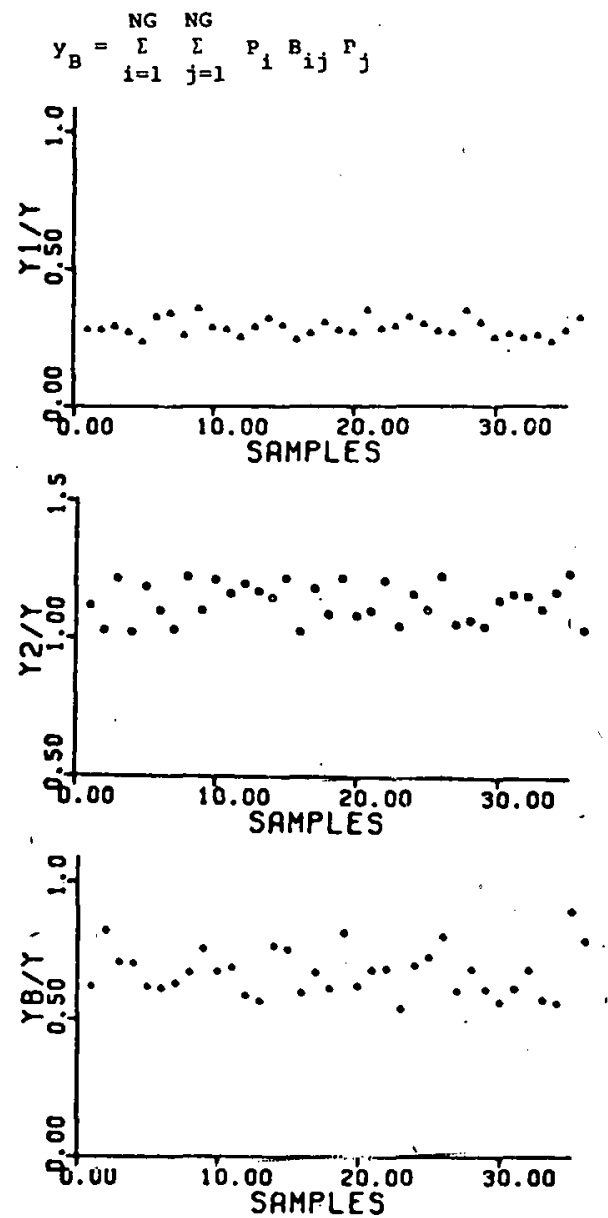

FIC. J: Cumparison of the Accuracies of the first ( $y$ ) and second ( $y$ ) order Taylor Expansion of system Losses $(y)$, with that of the B-coefficients $\left(y_{B}\right)$ Loss Formula. 
We have subjected the above loss formula to a similar error analysis approach with the following exceptions :

1) The expansion point is chosen to be one of the load levels from which the B-coefficients are calculated.

11) Only the real part of the voltages are allowed to vary within \pm 0.1 from their value at the expansion point. The imaginary part of the voltages are chosen such that the voltage levels at the system buses remain constant at their base values. Unrealistic operating points were discarded.

The above rostriction on the random numbers actually biases the samples strongly in favour of the B-coefficient loss formula, however as the results in Fig. (9) show, the quadratic Taylor expansion gives a much better result. The average error for the linear expansion was 708 . The average for the B-coefficients formula was 308 while that for the second order Taylor was 108 .

Similar comparisons between the linear approximation formulae presently in use with the linear expansion formulae introduced here have also been carried out. Because of lack of space the results are not presented here, but in general under unbiased conditions the linear expansions do much better than other linear approximation formulae such as the DC load flow method.

\section{CONCLUSIONS}

A systemat1c derivation of explicit approximation formulae for arbitrary dependent power network variables is introduced. The derivation can be based on a raylor series expansion of the dependent variables in terms of the known or controllable injections at the system buses, $z$ ( $P$ and $V^{2}$ at a PV bus, $P$ and $Q$ at $a P Q$ bus and $\mathrm{v}^{2}$ at the slack bus). It is also shown that explicit approximation formulae can be systematically derived from a Newton-Raphson load flow scheme.

The quadratic properties of the load flow equations in rectangular coordinates are exploited in these derivations as well as lil lie uulyoio of the approximation errors. It ia shown, for example, that for certain variables such as the real network. lusses and line flows, the linear expansion exror is always positive, a result which is useful in quantifying the nature of the approximation formula. A nore quantitative error estimation procedure for the linear expangion is also derived (equation(25)).

For the quadratic expansion it is more difficult to apriori quantify the error, although it is shown that these can be related to the error after one Newton-Raphson iteration.

In order to obtain a quantitative feel for the accuracy of the derived formulae, a large number of random simulations were carricd out. The simulation approach has an important feature which permitted us to make extensive load flow runs very rapidly and Inexpensively. Basically each component of the vector of complex bus voltages was randomly generated within a wide rance axound the expansion point. The corresponding injections and dependent variables were then computed and added to a set of ranclom samples unless the load flow turned out to be unrealistic (e.g. negative generation), in which case it was discarded.
The numerical results (see Table 1) indicate that the lincar expansion is consistently much more innacurate than the quadratic. It should be pointed out that the DC load flow is a special case of the IInear expansions presented here and results have shown the DC method to be less accurate. Although linear expansion formulae have been found to be acceptable in some applications, our results indicate that one should proceed with caution when applying these formulae.

This study also reveals that a sufficiently high accuracy can be obtained from quadratic expansions making them attractive in applications such as economic dispatch and security control. The quadratic formulae presented here do not require any special assumptions about the injections, as with B-coefficients formulae. The formulae derived in this paper are easily obtained from the Jacobian of the load flow equations (the Jacobian may be in either the general or the decoupled version), and are general in the sense that they include explicitly the real and reactive generations as well as the voltage magnitudes at the generation buses as independent variables. A comparison with a B-coefficient formula (see RESULTS) shows that even under biased conditions the general formula is much more accurate. Further studies on the application of those results to system security and economic dispatch are underway.

\section{REFERENCES}

[1] B. Stott "Review of Load Flow Calculation Methods," Proc. of IEEE, Vol. 62, No.7, 1974, pp. 959-972.

[2] W.S. Ku, P. Van Olinda, "Security and Voltago Applications of the Public Service Dispatch Center," PICA Conference, Denver, May 18-21, 1969.

[3] E. Hnyilicza, S.T.Y. Lee, F.C. Schweppe, "Steady State Security Regions: Set-Theoretic Approach," Pruc. 1975 PICA Conference, pp. 347-355, 1975.

[4] R. Fischl, J.A. De Maio, "Fast Identification of the Steady-state Security Regions for Power System Security Enhancement," Paper A76 076-0, Winter Power Meeting, N. York, 1976.

[5] J.C. Kaltenbach and L.P. Hajdu, "Optimal Corrective Rescheduling for Power Systems Security," leEe 'Ileu1s: on Dounr a pnaratus and systems, 1970 Summer Power Meeting, L.A., Calif., July 1970.

[6] H. Duran, " $\Lambda$ Simplex-Like Method for Solving the Optimum Power Flow Problem," Proc. 8th. PICA Conference in Minneapolis, Min.. 1973, pp.162-167.

(7) R. Lugtu, "Security Constrained Dispatch," Paper F 78 725-4, Sumer Power Meeting, Los Angeles, 1978 .

[8] E.F. Hill, W.D. Stevenson," A New Method of Letermining Loss coefficients" IEEE Trans, on Power Apparatus and Systems, Vol. PAS-87, No.7. July 1968, pp. 1548-1553.

(9) R.R. Shoult, W. Mack Grady, S. Helmick, "An Efficient Method for Computing Loss 5ormula Coefficients Based Upon the Method of Least Squares," paper F 78 739-5, Summer Power Meeting, Los Angeles, 1978.

[10] F.D. Galiana, "Voltage Power Limitations Imposed by the Network structure of a Power System", Proc.PICA,June 1975,pp. 365 to 363. 
[11] F.D.Gallana, J.Jarjis, "Feasibllity Constraints in Power Systems", Paper A 78 560-5, Sununer Power MeetIng. Los Angeles, July 1978.

(12) F.D.Galiana, H.Glavitsch, "A General Compensation Method for line Outage Load Flow Studies". Proc. PSCC conf., London, England, Sept.1975.

[13].1.0.Elgerd, "An Introduction to Electric Energy Systems". Mc Graw Hill 1971, p. 574.

Appendix A

Basic to the following derivation is the chain rule. Differentiating any variable of $x, x(x)$, with respect to $\underline{z}$ yields.

$$
-\frac{\partial x(\underline{x})}{\partial \underline{z}}=\frac{\partial x(\underline{x})}{\partial \underline{x}} \quad \frac{\partial \underline{x}}{\partial \underline{z}}=\frac{\partial \dot{x}(\underline{x})}{\partial \underline{x}}\left\{\frac{1}{2} \underline{L}^{-1}(\underline{x})\right\}
$$

where we have made use of equation (9). The following are detalled derivations of the first four terms of the Taylor expansion series in (16). Other terms can be derived in a similar fashion.

First Term

The first terin is $y\left(\underline{x}_{0}\right)$ which is trivially given

$$
y\left(\underline{x}_{0}\right)=\underline{x}_{0}^{T} \underline{R}_{y} \underline{x}_{0}
$$

second Term

From (15) it is easy to see that

$$
\frac{\partial y}{\partial \underline{x}}=2 \underline{x} \underline{T} \underline{y}
$$

Thus according to (Al).

$$
\frac{\partial \dot{y}}{\partial \underline{z}}=\underline{x}^{T} \underline{R}_{y} \underline{L}^{-1}(\underline{x})
$$

which yields the second term,

$$
\text { , second term }=\underline{B}^{\mathbf{T}} \Delta \underline{z}
$$

having used the simplified notation

$$
\text { and } \begin{aligned}
\underline{B}(\underline{x})=\left[\frac{\partial y}{\partial \underline{z}}\right]^{T}=\left[\underline{L}^{T}(\underline{x})\right]^{-1} \underline{R}_{y} \underline{x} \\
\underline{B}=\underline{B}\left(\underline{x}_{0}\right)
\end{aligned}
$$

\section{Third Term}

The third term is given by the expression

$$
\left.\frac{1}{2} \sum_{\substack{i=1 \\ j=1}}^{2 ! j-1} i \frac{\partial^{2} y}{\partial z_{i} \partial z}\right\}_{j=x_{0}} \Delta z_{i} \Delta z_{j} \text {. }
$$

The above expression with the partials evaluated at an arbitrary $x$ is denoted $\frac{T_{3}}{2}$ Then

$\mathbf{T}_{3}=\frac{\partial \mathbf{r}_{1}}{\partial \underline{z}} \cdot \underline{\underline{z}}$

where we have defined

$$
r_{1} \sum_{1=1}^{2 N-1} \frac{\partial y}{\partial z_{1}} \Delta z_{i}=\frac{\partial y}{\partial \underline{z}} \Delta \underline{z}_{*}=\underline{B}^{T}(\underline{x}) \Delta \underline{z}
$$

To compute the term $\frac{\partial r}{\partial \frac{z}{2}}$, consicler a differential chango in $x_{1}$ or equivalently in $\underline{\beta}^{T}(\underline{x})$.

$$
d \underline{B}^{T}(\underline{x})=d \underline{x}^{T} \stackrel{R}{Y}^{T} \underline{L}^{-1}(\underline{x})+\underline{x}^{T} \underline{R}_{y} d\left\{\underline{L}^{-1}(\underline{x})\right\}
$$

Noting that

$$
d\left(\underline{L}^{-1}(\underline{x})\right)=-\underline{L}^{-1}(\underline{x}) d \underline{L}(\underline{x}) \underline{L}^{-1}(\underline{x})
$$

one can write

$$
\underline{x}^{T} \underline{R}_{y} d\left\{\underline{L}^{-1}(\underline{x})\right\}=-\underline{B}^{T}(\underline{x}) d \underline{L}(\underline{x}) \underline{L}^{-1}(\underline{x})
$$

$$
=-d x^{T} J(B) L^{-1}(x)
$$

where we have made use of equation (13). Thus

$$
d \underline{\beta}^{T}(\underline{x})=d \underline{x}^{T}\left(\underline{R}_{y}-\underline{J}(\underline{\beta})\right) \underline{L}^{-1}(\underline{x})
$$

which gives

$$
\frac{\partial x}{\partial \underline{x}}=\Delta \underline{z}^{T}\left[\underline{L}^{T}(\underline{x})\right]^{-1}\left(R_{y}-\underline{J}(\underline{B})\right]
$$

Using equation( $A I$ ) one can reduce (A9) to

$$
T_{3}=\frac{1}{2} \Delta \underline{z}^{T}\left[\underline{L}^{T}(\underline{x})\right]^{-1}\left\{\underline{R}_{y}-\underline{J}(\underline{B}) \underline{L}^{-1}(\underline{x}) \Delta \underline{z}\right.
$$

so that

Third term $=\frac{1}{2}\left(T_{3}\right)^{x}=x_{0}$.

Fourth Term

The fourth term of equation (16) is given by

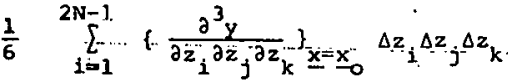

$$
\begin{aligned}
& j=1
\end{aligned}
$$

Following now a procedure very similar to the previous (but more intricate), one can show the following:

$$
\text { Fourth Term }=-\frac{1}{8} \underline{\alpha}^{T} \underline{J}(Y) \underline{a}
$$

where

$$
\underline{a}(\underline{x})=\underline{L}^{-2}(\underline{x}) \Delta \underline{z}
$$

and

$$
Y(\underline{x})=\left[\underline{L}^{T}(\underline{x})\right]^{-1}\{\underline{\underline{R}}-\underline{J}(\underline{\beta})\} \underline{a}(\underline{x})
$$

Using equation (22) it is easy to see that the fourth term just as the previous terms is an explicit function only of $z$ and nnt of $z$. Furtheimuse $1 t$ also breaks down into two terms one of which is the same as the third term.

\section{Acknowledgement}

This study was supported by the Division of Electric Energy. Systems, U.s. Dept. of Energy, under Contract No. EC-77-C-01-5011. 


\section{REFERENCES}

[1] Galiana, F.D., "Power Voltage Limitations Imposed by Network Structure of a Power System", PICA 1975, pp. $356 ; 363$.

[2] Galiana, F.D., Lee, K., "On the steady state stability of Power System", PICA 1977, pp. 201-210.

[3] Wilkinson, J.H., "The Algebraic Eigenvalue Problem", Oxford University Press, 1965, pp. 337.

[4] Gottfried, B.G., Weissman, J., "Introduction to Optimization Theory", Prentice-Hall Inc.

[5] Lasdon, L.S., "Optimization Theory for Large Systems", McMilian Company, 1970.

[6] Luenberger, D.G., "Introduction to Linear and NonLinear Programming", Addison-Wesley.

[7] Elgerd, I. Olle, "An Introduction to Electric Energy Systems", McGraw-Hill, 1971.

[8] Fletcher, R., Powe11, M.J.O., "A Rapidly Descent Method for Minimization", Computer Journal, Vol. 6, p. 163.

[9] Flotrher, R, "A New Approach to Variable Metric Algorithms", The Computer Journal, vol. 13, No. J, May 1970 , p. 317 .

[10] IBM System/360 Scientific Sub-routine Package (SSP).

[11] University of Waterloo, "An Optimization PackageQNMING", June, 1972.

[12] Strang, G., "Linear Algebra and Its Applications", Academic Press, 1976, pp. 231.

[13] Stevenson, W.D., "Elements of Power System Analysis", 2nd Edition, New York, McGraw-Hill, 1962.

[14] Stagg, G.W., and E1-Abiad, A.H., "Computer Methods in Power System Analysis, McGraw-Hill Book Co., 1968, pp. 284 .

[15] Bazaraa, M.S., Shetty, C.M., "Lecture Notes in Economics and Mathematical Systems", Springer-Verlag, 1976, pp. 13 . 
[16] Galiana, F.D., J. Jarjis, "Feasibility Constraints in Power Systems", IEEE Summer Paper Meeting, Los Angeles, Calif., July, 1973, Paper A78-560-5.

[17] "New-York 'City's Blackout: \$350 Million Drain," IEEE Spectrum, Vol. 15, No. 11, Nov. 1978, pp. 44-46.

[18] "Cost of Sypply Interruption to the Consumer," Electrical Review, Vol. 181, No. 15, Oct. 13, 1967, pp. 533-534.

[19] F.C'. Schweppe, "Power System '2000': Hierarchical Control Strategies," IEEE Spectrum, Vol. 15, No. 7, pp. 42-47, July 1978.

[20] T.E. Dy Liacco, "Real-Time Computer Control of Power Systems, "IEEE Proc., (Special issue: Computers in the Power Industry), Vol. 62, No. 7, pp. 884-891, July 1974.

[21] T.E. Dy Liacco, "Control of Power Systems via the Multi-Level Concept", System Research Center, Case Western Reserve University, SRC-68-19, Cleveland, 1968.

[22] T.E. Dy Liacco, B.F. Writy, D.A. Wheeler, "Automation of the CEI System for Security," IEEE Trans., Vol. PAS-91, May/June 1972.

[23] N.M. Peterson, J.P. Britton, T.J. Varney, "Advanced Applications System for the Wisconsin Electric Power Company Control Center," Power Industry Computer Applications, New Orleans, June 2-4, 1975.

[24] L.P. Hadju, D.W. Bree, and A.W. Brooks, "On-line Monitoring of Power System Security," Paper 70CP690-PWR, IEEE 1970 Summer Power Meeting, L.A., Calif.

[25] A.S. Debs, and A.R. Benson, "Security Assesment of Power System," Presented at the Engineering Foundation Conf. on System Engineering for Power, New England College, Henniker, N.H. Agust 17-22, 1975.

[26] L.P. Hadju, R. Podnore, and D.W. Bree, "Security Enhancement for Power Systems, "Final Report to Energy Research and Development Administration by Systems Control, Inc.,'Palo Alto, Calif., July 1975. 
[27] B. Stott, "Review of Load Flow Calculation Methods," Proc. IEEE, Vol. 62, pp. 916-929, July 1974.

[28] A.E. Sasson and H.M. Merrill, "Some Applications of Optimization Techniques to Power System Problems", Proc. IEEE, Vol. 62, pp. 959-972, July 1974.

[29] N.M. Paterson, W.F. Tinney, D.W. Bree, Jr., "Iterative Linear AC Power Flow Solution for Fast Approximate Outage Studies, "IEEE Transactions, Vol. PAS 91, sept./Oct. 1971 .

[30] F.D. Galiana, H. Glavisch, and A. Fiechter, "A General Compensation Method for the Study of Line Outages in Load Flow Problems", Proc. PSCC Conf., London, England, Sept. 1975.

[31] H.M. Happ, "Optimal Power Dispatch", Presented at ERDA Conference on Systems Engineering for Power: Status and Prospects, Henniker, New Hampshire, August, 1975 .

[32] Proceedings of E.P.R.I. Symposium on Parallel and Distributed Processing in Power Systems, Palo Alto, California, 1978.

[33] M. Manas and J. Nedoma, "Finding All Vertices of a Convex Polyhedral", Numerische Mathematik, Vol. 12, pp. 226-229, 1968 .

[34] T.H. Matheiss, "An Algorithm for Determining Irrelevant Constraints and All Vertices in Systems of Linear Inequalities", Operations Research, Vol. 21, pp. 247-260. 1973 .

[35] M.L. Balinski, "An Algorithm for Finding All Vertices of Convex Polyhedral Sets", SIAM Journal of Applied Mathematics, Vol. 9, pp. 72-88, 1961.

[36] J.A. De Maio, R. Fischl, "Fast Identification of the Steady-State Security Region for Power system Security Enhancement", Paper No. A-76-076-0, IEEE Winter Power Meeting, January, 1976 . 


\section{United States}

Department of Energy

Washington, DC 20545

Official Business

Penalty for Private Use, $\$ 300$

DOE-350

\section{AIL}

oak Ridge, IN 37830 